\title{
Projections of Education Statistics to 2020
}

Thirty-ninth Edition

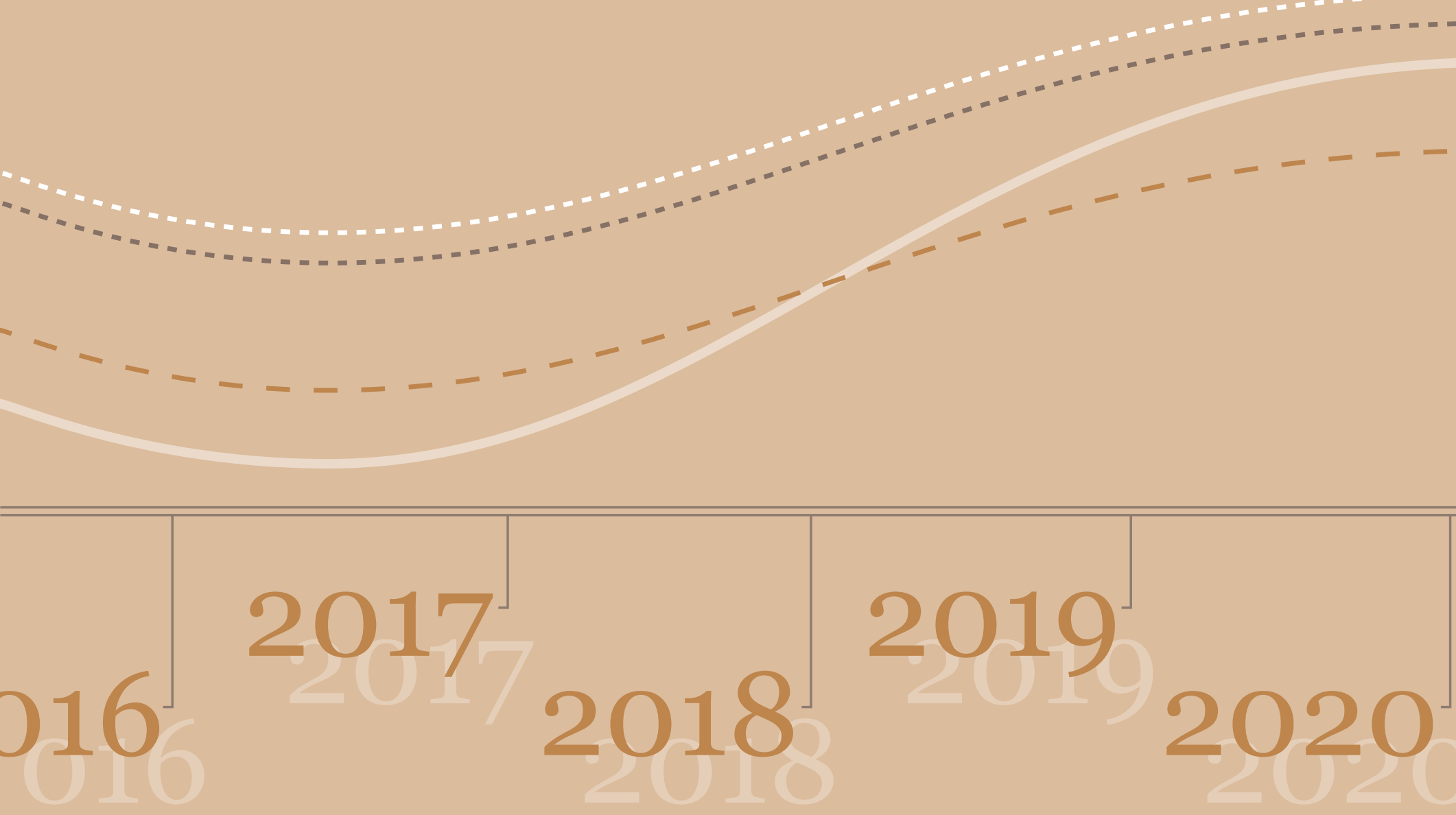





\title{
Projections of Education Statistics to 2020
}

\author{
Thirty-ninth Edition
}

\section{September 2011}

\author{
William J. Hussar \\ National Center for Education Statistics \\ Tabitha M. Bailey \\ IHS Global Insight
}




\section{U.S. Department of Education}

Arne Duncan

Secretary

Institute of Education Sciences

John Q. Easton

Director

\section{National Center for Education Statistics}

Jack Buckley

Commissioner

The National Center for Education Statistics (NCES) is the primary federal entity for collecting, analyzing, and reporting data related to education in the United States and other nations. It fulfills a congressional mandate to collect, collate, analyze, and report full and complete statistics on the condition of education in the United States; conduct and publish reports and specialized analyses of the meaning and significance of such statistics; assist state and local education agencies in improving their statistical systems; and review and report on education activities in foreign countries.

NCES activities are designed to address high-priority education data needs; provide consistent, reliable, complete, and accurate indicators of education status and trends; and report timely, useful, and high-quality data to the U.S. Department of Education, the Congress, the states, other education policymakers, practitioners, data users, and the general public. Unless specifically noted, all information contained herein is in the public domain.

We strive to make our products available in a variety of formats and in language that is appropriate to a variety of audiences. You, as our customer, are the best judge of our success in communicating information effectively. If you have any comments or suggestions about this or any other NCES product or report, we would like to hear from you. Please direct your comments to

NCES, IES, U.S. Department of Education 1990 K Street NW

Washington, DC 20006-5651

\section{September 2011}

The NCES Home Page address is http://nces.ed.gov.

The NCES Publications and Products address is http://nces.ed.gov/pubsearch.

This report was prepared in part under Contract No. ED-08-DO-0087 with IHS Global Insight. Mention of trade names, commercial products, or organizations does not imply endorsement by the U.S. Government.

\section{Suggested Citation}

Hussar, W.J., and Bailey, T.M. (2011). Projections of Education Statistics to 2020 (NCES 2011-026). U.S. Department of Education, National Center for Education Statistics. Washington, DC: U.S. Government Printing Office.

\section{For ordering information on this report, write to}

ED Pubs, U.S. Department of Education

P.O. Box 22207

Alexandria, VA 22304

or call toll free 1-877-4ED-PUBS or order online at http://www.edpubs.gov.

\section{Content Contact}

William J. Hussar

(202) 502-7359

william.hussar@ed.gov 


\section{Foreword}

Projections of Education Statistics to 2020 is the 39th report in a series begun in 1964. It includes statistics on elementary and secondary schools and postsecondary degree-granting institutions. This report provides revisions of projections shown in Projections of Education Statistics to 2019. Included are projections of enrollment, graduates, teachers, and expenditures to the year 2020 .

In addition to projections at the national level, the report includes projections of public elementary and secondary school enrollment and public high school graduates to the year 2020 at the state level. The projections in this report were produced by the National Center for Education Statistics (NCES) to provide researchers, policy analysts, and others with state-level projections developed using a consistent methodology. They are not intended to supplant detailed projections prepared for individual states.

Assumptions regarding the population and the economy are the key factors underlying the projections of education statistics. NCES projections do not reflect changes in national, state, or local education policies that may affect education statistics.
Appendix A of this report outlines the projection methodology and describes the models and assumptions used to develop the national and state projections. The enrollment models use enrollment data and population estimates and projections from NCES and the U.S. Census Bureau. The models are based on the mathematical projection of past data patterns into the future. The models also use projections of economic variables from IHS Global Insight, an economic forecasting service.

The projections presented in this report are based on the 2000 census and assumptions for the fertility rate, internal migration, net immigration, and mortality rate from the Census Bureau. For further information, see appendix A.

\section{Val Plisko, Associate Commissioner}

Early Childhood, International, and Crosscutting Studies Division 
This page intentionally left blank. 


\section{Contents}

$\begin{array}{r}\text { Page } \\ \hline\end{array}$

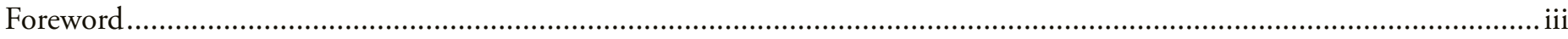

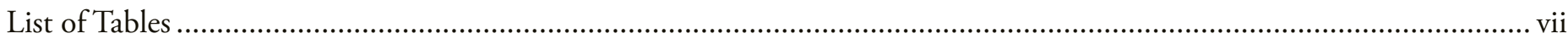

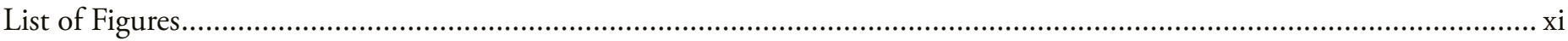

About This Report.

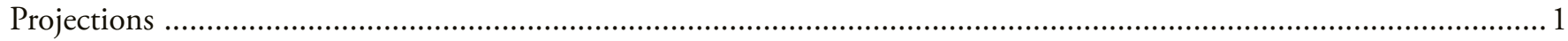

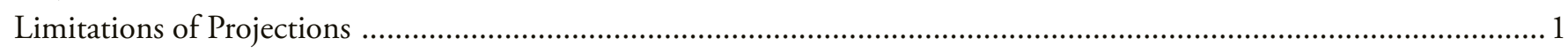

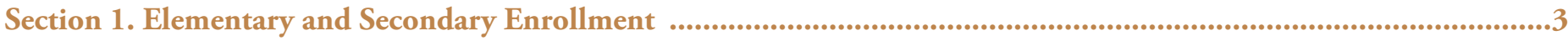

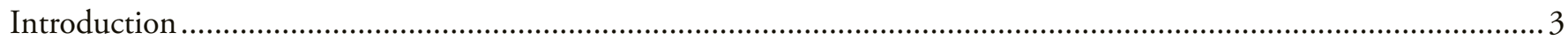

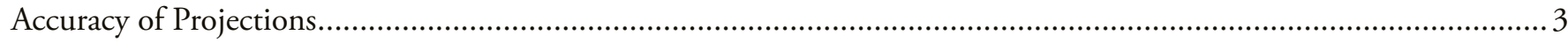

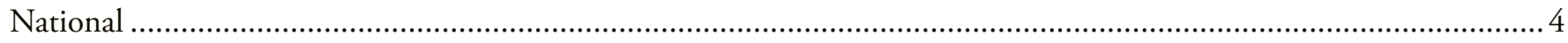

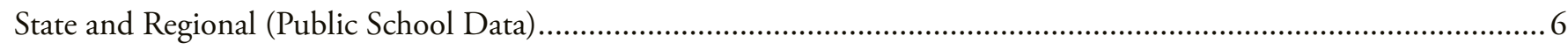

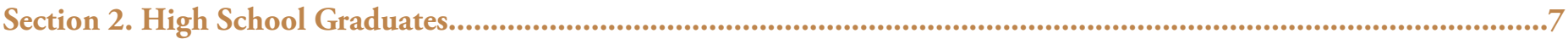

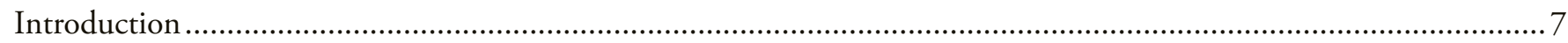

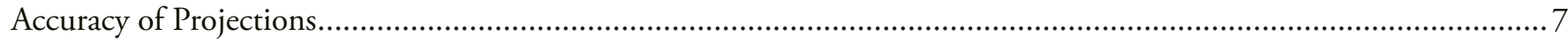

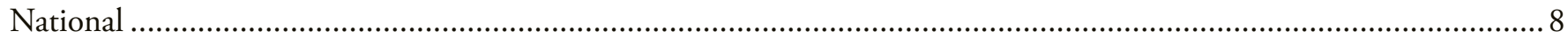

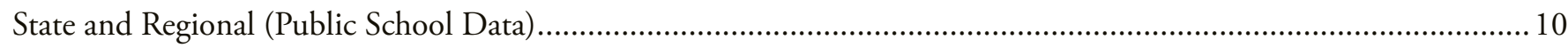

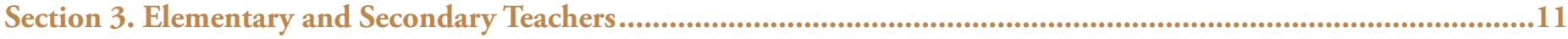

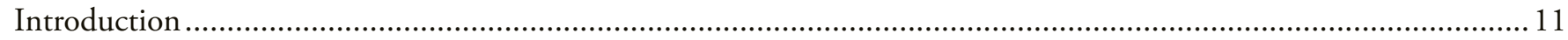

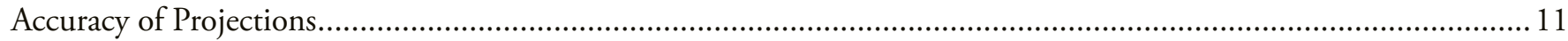

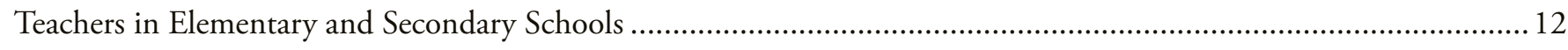

Section 4. Expenditures for Public Elementary and Secondary Education ......................................................15

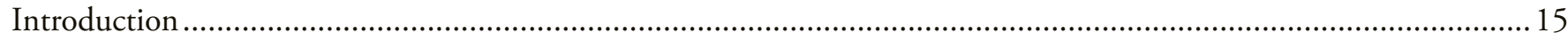

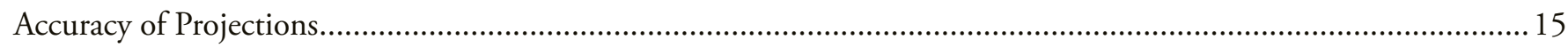

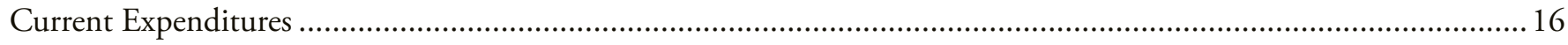

Section 5. Enroll ment in Postsecondary Degree-Granting Institutions ........................................................ 19

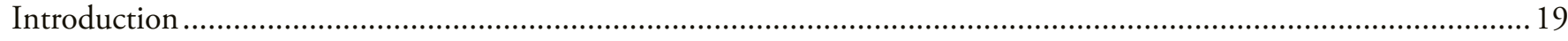

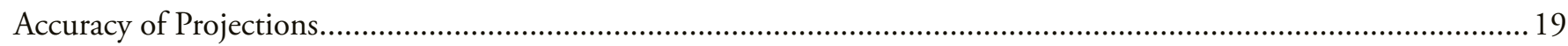

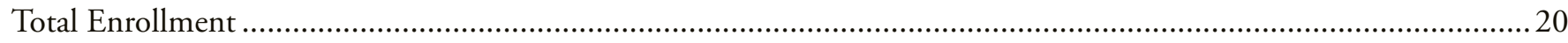

Enrollment by Selected Characteristics and Control

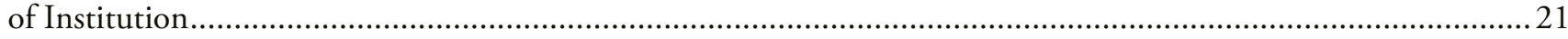

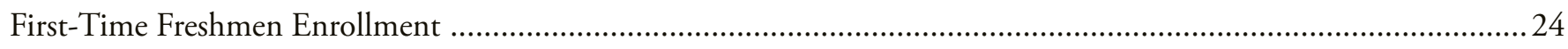




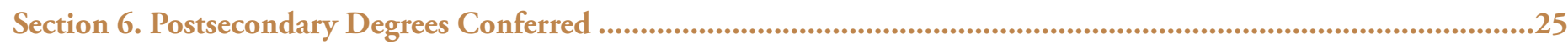

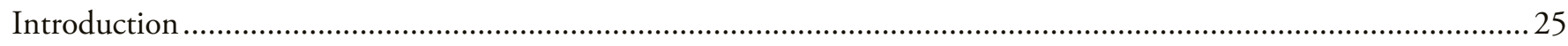

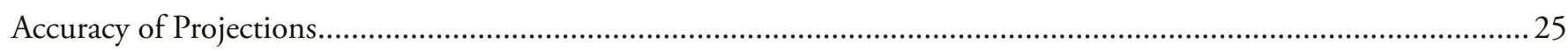

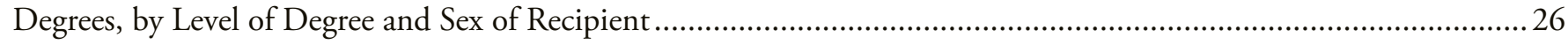

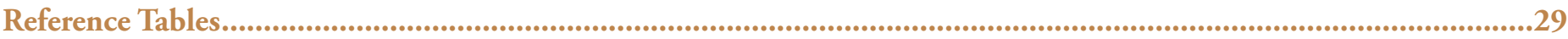

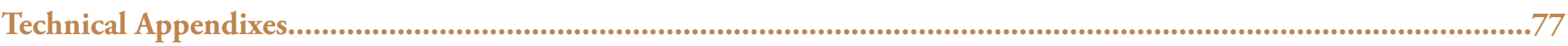

Appendix A. Introduction to Projection Methodology ….................................................................................................78

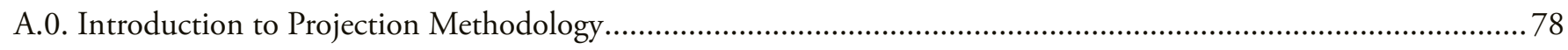

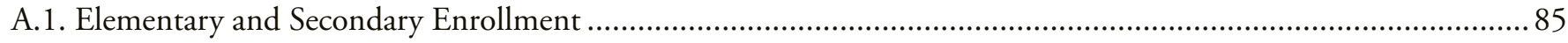

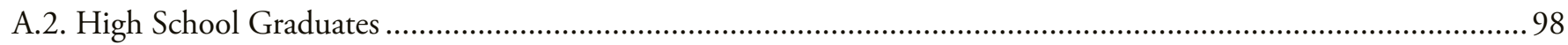

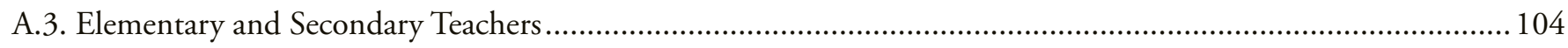

A.4. Expenditures for Public Elementary and Secondary Education...................................................................112

A.5. Enrollment in Postsecondary Degree-Granting Institutions..................................................................... 116

A.6. Postsecondary Degrees Conferred.......................................................................................................... 135

Appendix B. Supplementary Tables ........................................................................................................................139

Appendix C. Data Sources ..........................................................................................................................................147

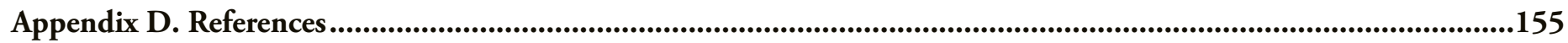

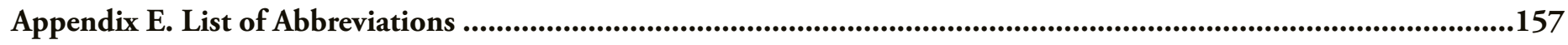

Appendix F. Glossary ..................................................................................................................................................159 


\section{List of Tables}

Table

1. Actual and projected numbers for enrollment in grades PK-12, PK-8, and 9-12 in elementary and secondary schools, by control of school: Fall 1995 through fall 2020

2. Actual and projected numbers for enrollment in public elementary and secondary schools, by grade: Fall 1995 through fall 2020 ...

3. Actual and projected numbers for enrollment in public elementary and secondary schools, by race/ethnicity: Fall 1995 through fall 2020

4. Actual and projected numbers for enrollment in grades PK-8 in public elementary and secondary schools, by race/ethnicity: Fall 1995 through fall 2020.

5. Actual and projected numbers for enrollment in grades 9-12 in public schools, by race/ethnicity: Fall 1995 through fall 2020

6. Actual and projected numbers for enrollment in grades PK-12 in public elementary and secondary schools, by region and state: Fall 2002 through fall 2020 .....

7. Actual and projected percentage changes in grades PK-12 enrollment in public elementary and secondary schools, by region and state: Selected years, fall 2002 through fall 2020.

8. Actual and projected numbers for enrollment in grades PK-8 in public elementary and secondary schools, by region and state: Fall 2002 through fall 2020

9. Actual and projected percentage changes in grades PK-8 enrollment in public elementary and secondary schools, by region and state: Selected years, fall 2002 through fall 2020

10. Actual and projected numbers for enrollment in grades 9-12 in public schools, by region and state: Fall 2002 through fall 2020

11. Actual and projected percentage changes in grades 9-12 enrollment in public schools, by region and state: Selected years, fall 2002 through fall 2020 .

12. Actual and projected numbers for high school graduates, by control of school: School years 1995-96 through 2020-21

13. Actual and projected numbers for public high school graduates, by race/ethnicity: School years 1995-96 through 2020-21

14. Actual and projected numbers for public high school graduates, by region and state: School years 2002-03 through 2020-21.....

15. Actual and projected percentage changes in public high school graduates, by region and state: Selected school years, 2002-03 through 2020-21.

16. Actual and projected numbers for elementary and secondary teachers and elementary and secondary new teacher hires, by control of school: Fall 1995 through fall 2020 .

17. Actual and projected numbers for the pupil/teacher ratios in elementary and secondary schools, by control of school: Fall 1995 through fall 2020.

18. Actual and projected numbers for current expenditures and current expenditures per pupil in fall enrollment for public elementary and secondary education: 1995-96 through 2020-21.

19. Actual and projected numbers for current expenditures and current expenditures per pupil in average daily attendance (ADA) for public elementary and secondary education: School years 1995-96 through 2020-21 .......56

20. Actual and projected numbers for total enrollment in all postsecondary degree-granting institutions, by sex, attendance status, and control of institution: Fall 1995 through fall 2020....... 
21. Actual and projected numbers for total enrollment in all postsecondary degree-granting institutions, by

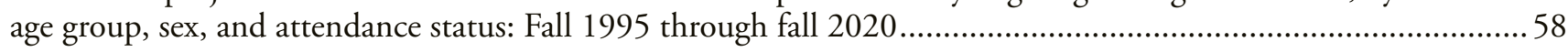

22. Actual and projected numbers for enrollment in all postsecondary degree-granting institutions, by sex and attendance status: Fall 1995 through fall 2020

23. Actual and projected numbers for enrollment in public 4-year postsecondary degree-granting institutions, by sex and attendance status: Fall 1995 through fall 2020 .....

24. Actual and projected numbers for enrollment in public 2-year postsecondary degree-granting institutions, by sex and attendance status: Fall 1995 through fall 2020

25. Actual and projected numbers for enrollment in private 4-year postsecondary degree-granting institutions, by sex and attendance status: Fall 1995 through fall 2020 .

26. Actual and projected numbers for enrollment in private 2-year postsecondary degree-granting institutions, by sex and attendance status: Fall 1995 through fall 2020 .

27. Actual and projected numbers for undergraduate enrollment in all postsecondary degree-granting institutions, by sex, attendance status, and control of institution: Fall 1995 through fall 2020

28. Actual and projected numbers for postbaccalaureate enrollment in all postsecondary degree-granting institutions, by sex, attendance status, and control of institution: Fall 1995 through fall 2020

29. Actual and projected numbers for enrollment in all postsecondary degree-granting institutions, by race/ethnicity: Fall 1995 through fall 2020

30. Actual and projected numbers for first-time freshmen fall enrollment in all postsecondary degree-granting institutions, by sex: Fall 1995 through fall 2020.

31. Actual and projected numbers for full-time-equivalent enrollment in all postsecondary degree-granting institutions, by control and type of institution: Fall 1995 through fall 2020

32. Actual and projected numbers for associate's degrees conferred by postsecondary degree-granting institutions, by sex of recipient: 1995-96 through 2020-21

33. Actual and projected numbers for bachelor's degrees conferred by postsecondary degree-granting institutions, by sex of recipient: 1995-96 through 2020-21.....

34. Actual and projected numbers for master's degrees conferred by postsecondary degree-granting institutions, by sex of recipient: 1995-96 through 2020-21

35. Actual and projected numbers for doctor's degrees conferred by postsecondary degree-granting institutions, by sex of recipient: 1995-96 through 2020-21 75

36. Actual and projected numbers for first-professional degrees conferred by postsecondary degree-granting institutions, by sex of recipient: 1995-96 through 2020-21

\section{Text Tables}

A. Mean absolute percentage errors (MAPEs) of enrollment projections, by lead time, control of school, and grade in elementary and secondary schools: 2011.

B. Mean absolute percentage errors (MAPEs) of projections of high school graduates, by lead time and control of school: 2011 .....

C. Mean absolute percentage errors (MAPEs) of projections of number of public elementary and secondary teachers, by lead time: 2011

D. Mean absolute percentage errors (MAPEs) of projections for total and per pupil current expenditures for public elementary and secondary education, by lead time: 2011.

E. Mean absolute percentage errors (MAPEs) of projected enrollment in postsecondary degree-granting institutions, by lead time, sex, and level of institution: 2011 .

F. Mean absolute percentage errors (MAPEs) of projected enrollment in postsecondary degree-granting insitutions, by lead time and race/ethnicity: 2011 


\section{Appendix A. Introduction to Projection Methodology}

A-1. Summary of forecast assumptions to 2020 .....

A-2. Mean absolute percentage errors (MAPEs) by lead time for selected statistics in all public elementary and secondary schools and postsecondary degree-granting institutions: 2011.

A-3. Example of constructing mean absolute percentage errors, part 1

A-4. Example of constructing mean absolute percentage errors, part 2.

A-5. Actual and projected national public school grade progression rates: Fall 2008, and fall 2009 through fall 2020 .

A-6. Actual and projected national enrollment rates in public schools, by grade level: Fall 2008, and fall 2009 through fall 2020

A-7. Mean absolute percentage errors (MAPEs) for projected prekindergarten-12 enrollment in public elementary and secondary schools, by lead time, region, and state: 2011 ......

A-8. Mean absolute percentage errors (MAPEs) for projected prekindergarten-8 enrollment in public elementary and secondary schools, by lead time, region, and state: 2011.

A-9. Mean absolute percentage errors (MAPEs) for projected grades 9-12 enrollment in public schools, by lead time, region, and state: 2011

A-10. Mean absolute percentage errors (MAPEs) for the projected number of high school graduates in public schools, by lead time, region, and state: 2011

A-11. Estimated equations and model statistics for public elementary and secondary teachers

A-12. Percentage distribution of full-time and part-time school teachers, by age, control of school, and teaching status: $2007-08$.

A-13. Percentage distribution of full-time and part-time newly hired teachers, by age and control of school: Selected years, 1987-88 through 2007-08.

A-14. Actual and projected continuation rates of full-time and part-time school teachers, by age and control of school: Selected years, 1993-94 to 1994-95 through 2020-21 to 2021-22.....

A-15. Estimated equations and model statistics for current expenditures per pupil in fall enrollment for public elementary and secondary schools, and education revenue from state sources per capita.

A-16. Actual and projected numbers for college enrollment rates, by sex, attendance status, and age: Fall 2009, fall 2015, and fall 2020

A-17. Estimated equations and model statistics for full-time and part-time college enrollment rates of men

A-18. Estimated equations and model statistics for full-time and part-time college enrollment rates of women ............ 124

A-19. Actual and projected percentages of full-time students at postsecondary degree-granting institutions, by sex, age group, student level, and institution level: Fall 2009, and fall 2010 through fall 2020

A-20. Actual and projected percentages of part-time students at postsecondary degree-granting institutions, by sex, age group, level enrolled, and level of institution: Fall 2009, and fall 2010 through fall 2020

A-21. Actual and projected enrollment in public postsecondary degree-granting institutions as a percent of total postsecondary enrollment, by sex, attendance status, level enrolled, and level of institution: Fall 2009, and fall 2010 through fall 2020

A-22. Estimated equations and model statistics for full-time and part-time college enrollment rates of White men ...... 127

A-23. Estimated equations and model statistics for full-time and part-time college enrollment rates of White women.. 128

A-24. Estimated equations and model statistics for full-time and part-time college enrollment rates of Black men........ 129

A-25. Estimated equations and model statistics for full-time and part-time college enrollment rates of Black women ... 130

A-26. Estimated equations and model statistics for full-time and part-time college enrollment rates of Hispanic men .. 131

A-27. Estimated equations and model statistics for full-time and part-time college enrollment rates of Hispanic women. 
A-28. Estimated equations and model statistics for full-time and part-time college enrollment rates of Asian/Pacific

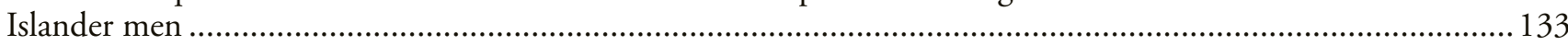

A-29. Estimated equations and model statistics for full-time and part-time college enrollment rates of Asian/Pacific Islander women....

A-30. Estimated equations and model statistics for degrees conferred, by degree level and sex

\section{Appendix B. Supplementary Tables}

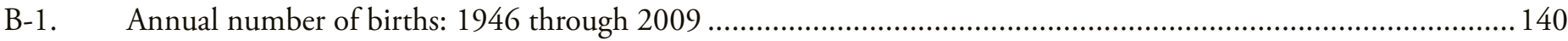

B-2. Actual and projected prekindergarten- and kindergarten-age populations, by age: 1995 through 2020 .............. 141

B-3. Actual and projected school-age populations, by selected ages: 1995 through 2020 .......................................142

B-4. Actual and projected college-age populations, by selected ages: 1995 through 2020 .......................................143

B-5. Actual and projected fall enrollment in public elementary and secondary schools, change in fall enrollment from previous year, resident population, and fall enrollment as a ratio of the population: School years 1995-96 through 2020-21

B-6. Actual and projected macroeconomic measures of the economy: School years 1995-96 through 2020-21 .........145 


\section{List of Figures}

Figure

1. Actual and projected numbers for enrollment in elementary and secondary schools, by grade level: Fall 1995 through fall 2020.

2. Actual and projected numbers for enrollment in elementary and secondary schools, by control of school: Fall 1995 through fall 2020.

3. Actual and projected numbers for enrollment in public elementary and secondary schools, by race/ethnicity: Fall 1995 through fall 2020.

4. Projected percentage change in enrollment in public elementary and secondary schools, by state: Fall 2008 through fall 2020 .....

5. Actual and projected numbers for enrollment in public elementary and secondary schools, by region: Fall 2002, fall 2008, and fall 2020

6. Actual and projected numbers for high school graduates, by control of school: School years 1995-96 through 2020-21. 8

7. Actual and projected numbers for public high school graduates, by race/ethnicity: School years 1995-96 through 2020-21

8. Projected percentage change in the number of public high school graduates, by state: School years 2007-08 through 2020-21.

9. Actual and projected numbers for public high school graduates, by region: School years 2002-03, 2007-08, and 2020-21.

10. Actual and projected numbers for elementary and secondary teachers, by control of school: Fall 1995 through fall 2020

11. Actual and projected numbers for the pupil/teacher ratios in elementary and secondary schools, by control of school: Fall 1995 through fall 2020

12. Actual and projected numbers for elementary and secondary new teacher hires, by control of school: Fall 1999, fall 2008, and fall 2020.

13. Actual and projected current expenditures for public elementary and secondary schools (in constant 2008-09 dollars): School years 1995-96 through 2020-21

14. Actual and projected current expenditures per pupil in fall enrollment in public elementary and secondary schools (in constant 2008-09 dollars): School years 1995-96 through 2020-21 17

15. Actual and projected population numbers for 18- to 24-year-olds and 25- to 29-year-olds: 1995 through $2020 \ldots . . . . .19$

16. Actual and projected numbers for total enrollment in all postsecondary degree-granting institutions: Fall 1995 through fall 2020.

17. Actual and projected numbers for enrollment in all postsecondary degree-granting institutions, by age group: Fall 1995, fall 2009, and fall 2020

18. Actual and projected numbers for enrollment in all postsecondary degree-granting institutions, by sex: Fall 1995 through fall 2020

19. Actual and projected numbers for enrollment in all postsecondary degree-granting institutions, by attendance status: Fall 1995 through fall 2020.

20. Actual and projected numbers for undergraduate and postbaccalaureate enrollment in all postsecondary degree-granting institutions: Fall 1995 through fall 2020 
21. Actual and projected numbers for enrollment in all postsecondary degree-granting institutions, by race/ethnicity: Fall 1995 through fall 2020

22. Actual and projected numbers for enrollment in all postsecondary degree-granting institutions, by control of institution: Fall 1995 through fall 2020

23. Actual and projected numbers for total first-time freshmen enrollment in all postsecondary degree-granting institutions, by sex: Fall 1995 through fall $2020 .$.

24. Actual and projected numbers for associate's degrees conferred by postsecondary degree-granting institutions, by sex of recipient: 1995-96 through 2020-21

25. Actual and projected numbers for bachelor's degrees conferred by postsecondary degree-granting institutions, by sex of recipient: 1995-96 through 2020-21. .26

26. Actual and projected numbers for master's degrees conferred by postsecondary degree-granting institutions, by sex of recipient: 1995-96 through 2020-21

27. Actual and projected numbers for doctor's degrees conferred by postsecondary degree-granting institutions, by sex of recipient: $1995-96$ through $2020-21$.

28. Actual and projected numbers for first-professional degrees conferred by postsecondary degree-granting institutions, by sex of recipient: 1995-96 through 2020-21 


\section{About This Report}

\section{PROJECTIONS}

This edition of Projections of Education Statistics provides projections for key education statistics, including enrollment, graduates, teachers, and expenditures in elementary and secondary public and private schools, as well as enrollment and degrees conferred at postsecondary degree-granting institutions. Included are national data on enrollment and graduates for the past 15 years and projections to the year 2020. Also included are state-level data on enrollment in public elementary and secondary schools and public high schools from 2002, with projections to 2020. This report is organized by the level of schooling with sections 1,2, 3, and 4 covering aspects of elementary and secondary education and sections 5 and 6 covering aspects of postsecondary education.

There are a number of limitations in projecting some statistics. First, state-level data on enrollment and graduates in private elementary and secondary schools and on enrollment and degrees conferred in postsecondary degree-granting institutions are not included. Further research and model development are needed to develop reliable projections of private school enrollment and graduates by state. Neither the actual numbers nor the projections of public and private elementary and secondary school enrollment include homeschooled students because more data are required to develop reliable projections.

Similar methodologies were used to obtain a uniform set of projections for each of the 50 states and the District of Columbia. These projections are further adjusted to agree with the national projections of public elementary and secondary school enrollment and public high school graduates contained in this report.

There were changes in how the exponential smoothing forecasting technique was used in this edition of Projections of Education Statistics, compared to how it had been used in previous editions. Exponential smoothing is used in the projections for elementary and secondary enrollments, high school graduates, new teacher hires, postsecondary degreegranting enrollments, and degrees conferred. In prior editions of Projections of Education Statistics, the smoothing formula used fixed coefficients and the new methodology uses variable coefficients. There has been no systematic examination to determine whether the projections computed using the new method are comparable to those using the prior method. For information about exponential smoothing used in Projections, see appendix A.0.

The summary of projections provides highlights of the national and state data, while the reference tables and figures present more detail. All calculations within Projections of Education Statistics are based on unrounded estimates. Therefore, the reader may find that a calculation, such as a difference or percentage change, cited in the text or figure may not be identical to the calculation obtained by using the rounded values shown in the accompanying tables.

Appendix A describes the methodology and assumptions used to develop the projections; appendix B presents supplementary tables; appendix $\mathrm{C}$ describes data sources; appendix $\mathrm{D}$ is a list of abbreviations; appendix $\mathrm{E}$ presents a list of the references; and appendix $\mathrm{F}$ is a glossary of terms.

\section{LIMITATIONS OF PROJECTIONS}

Projections of a time series usually differ from the final reported data due to errors from many sources, such as the properties of the projection methodologies, which depend on the validity of many assumptions.

The mean absolute percentage error is one way to express the forecast accuracy of past projections. This measure expresses the average of the absolute values of errors in percentage terms, where errors are the differences between past projections and actual data. For example, based on past editions of Projections of Education Statistics, the mean absolute percentage errors of public school enrollment in grades prekindergarten-12 for lead times of $1,2,5$, and 10 years were $0.3,0.6,1.3$, and 2.4 percent, respectively. In contrast, mean absolute percentage errors of private school enrollment in grades prekindergarten through 8 for lead times of 1, 2, 5, and 10 years were 3.5, 4.9, 9.2, and 7.9 percent, respectively. For more information on mean absolute percentage errors, see table A-2 in appendix A. 
This page intentionally left blank. 


\section{Section 1 \\ Elementary and \\ Secondary Enrollment}

\section{INTRODUCTION}

Total public and private elementary and secondary school enrollment was 55 million in fall 2008, representing an 8 percent increase since fall 1995. Between fall 2008, the last year of actual public school data, and fall 2020, a further increase of 5 percent is expected, with increases projected in public schools and decreases in private schools. Increases in public school enrollment are expected for Blacks, Hispanics, Asians/Pacific Islanders, and American Indians/Alaska Natives, and a decrease is expected for Whites. Increases in public school enrollment are expected in the Midwest, South, and West, and a decrease is expected in the Northeast.

\section{Factors affecting the projections}

The grade progression rate method was used to project school enrollments. This method assumes that future trends in factors affecting enrollments will be consistent with past patterns. It implicitly includes the net effect of factors such as dropouts, deaths, nonpromotion, transfers to and from public schools, and, at the state level, migration. See appendixes A.0 and A.1 for more details.

\section{Factors that were not considered}

The projections do not assume changes in policies or attitudes that may affect enrollment levels. For example, they do not account for changing state and local policies on prekindergarten $(\mathrm{PK})$ and kindergarten programs. Continued expansion of these programs could lead to higher enrollments at the elementary school level. Projections also exclude the number of students who are homeschooled because more data are needed.

\section{Accuracy of Projections}

An analysis of projection errors from the past 27 editions of Projections of Education Statistics indicates that the mean absolute percentage errors (MAPEs) for lead times of 1,2, 5, and 10 years out for projections of public school enrollment in grades PK-12 were $0.3,0.6,1.3$, and 2.4 percent, respectively. For the 1-year-out prediction, this means that the methodology used by the National Center for Education Statistics (NCES) has produced projections that have, on average, deviated from actual observed values by 0.3 percent. For projections of public school enrollment in grades PK-8, the MAPEs for lead times of $1,2,5$, and 10 years out were $0.4,0.7,1.3$, and 3.2 percent, respectively, while the MAPEs for projections of public school enrollment in grades $9-12$ were $0.3,0.6,1.3$, and 2.3 percent, respectively, for the same lead times. An analysis of projection errors from the past nine editions of Projections of Education Statistics indicates that the mean absolute percentage errors (MAPEs) for lead times of 1, 2, 5, and 10 years out for projections of private school enrollment in grades PK-12 were 3.4, 4.6, 7.7, and 6.3 percent, respectively. For projections of private school enrollment in grades PK-8, the MAPEs for lead times of $1,2,5$, and 10 years out were 3.5, 4.9, 9.2, and 7.9 percent, respectively, while the MAPEs for projections of private school enrollment in grades 9-12 were 3.0, 3.8, 2.8, and 1.3 percent, respectively, for the same lead times. For more information, see table A-2 in appendix A. 
Total elementary and secondary enrollment

$\triangle$ increased 8 percent between 1995 and 2008; and

$\Delta$ is projected to increase an additional 5 percent between 2008 and 2020.

Enrollment in prekindergarten through grade 8

$\triangle$ increased 4 percent between 1995 and 2008; and

$\Delta$ is projected to increase an additional 8 percent between 2008 and 2020.

Enrollment in grades 9-12

$\triangle$ increased 19 percent between 1995 and 2008; and

$\nabla$ is projected to decrease less than 1 percent between 2008 and 2020 .

For more information:

Tables 1 and 2
Figure 1. Actual and projected numbers for enrollment in elementary and secondary schools, by grade level: Fall 1995 through fall 2020

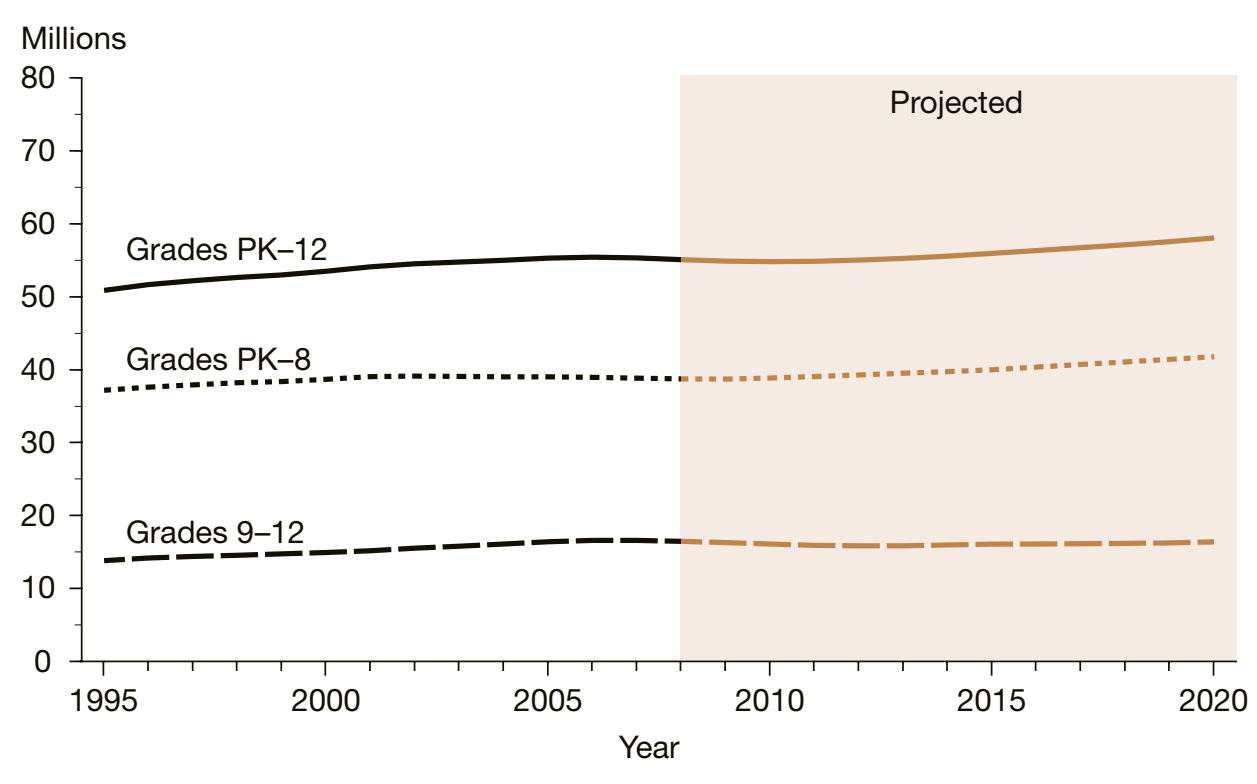

NOTE: PK = prekindergarten. Enrollment numbers for prekindergarten through 12th grade and prekindergarten through 8th grade include private nursery and prekindergarten enrollment in schools that offer kindergarten or higher grades. Since the biennial Private School Universe Survey (PSS) is collected in the fall of odd numbered years, private school numbers for alternate years are estimated based on data from the PSS. The private school numbers for 2009 are actual numbers from 2009-10 PSS. Some data have been revised from previously published figures. Mean absolute percentage errors of selected education statistics can be found in table A-2, appendix A.

SOURCE: U.S. Department of Education, National Center for Education Statistics, Common Core of Data (CCD), "State Nonfiscal Survey of Public Elementary/Secondary Education," 1995-96 through 2008-09; Private School Universe Survey (PSS), selected years 1995-96 through 2009-10; and National Elementary and Secondary Enrollment Model, 1972-2008. (This figure was prepared February 2011.) 
Figure 2. Actual and projected numbers for enrollment in elementary and secondary schools, by control of school: Fall 1995 through fall 2020

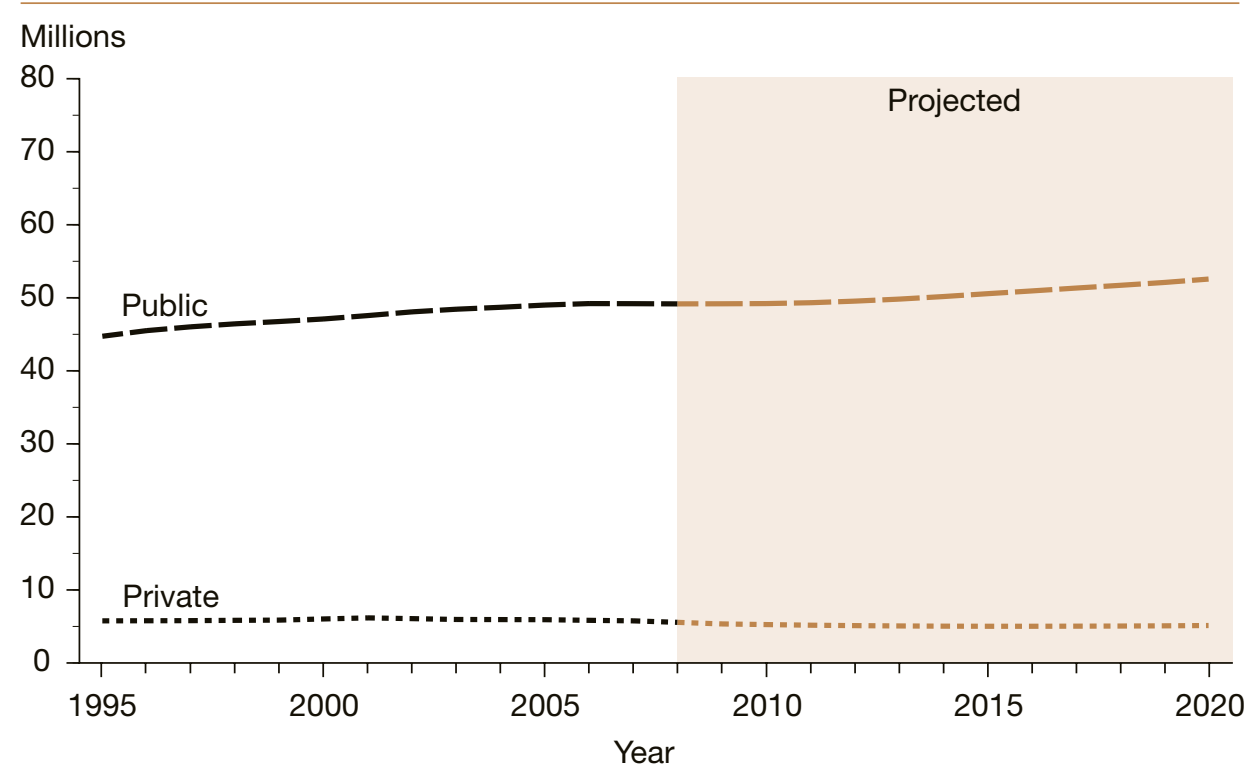

NOTE: Private school numbers include private nursery and prekindergarten enrollment in schools that offer kindergarten or higher grades. Since the biennial Private School Universe Survey (PSS) is collected in the fall of odd numbered years, private school numbers for alternate years are estimated based on data from the PSS. The private school numbers for 2009 are actual numbers from 2009-10 PSS. Some data have been revised from previously published figures. Mean absolute percentage errors of selected education statistics can be found in table A-2, appendix A.

SOURCE: U.S. Department of Education, National Center for Education Statistics, Common Core of Data (CCD), "State Nonfiscal Survey of Public Elementary/Secondary Education," 1995-96 through 2008-09; Private School Universe Survey (PSS), selected years 1995-96 through 2009-10; and National Elementary and Secondary Enrollment Model, 1972-2008. (This figure was prepared February 2011.)

Figure 3. Actual and projected numbers for enrollment in public elementary and secondary schools, by race/ethnicity: Fall 1995 through fall 2020

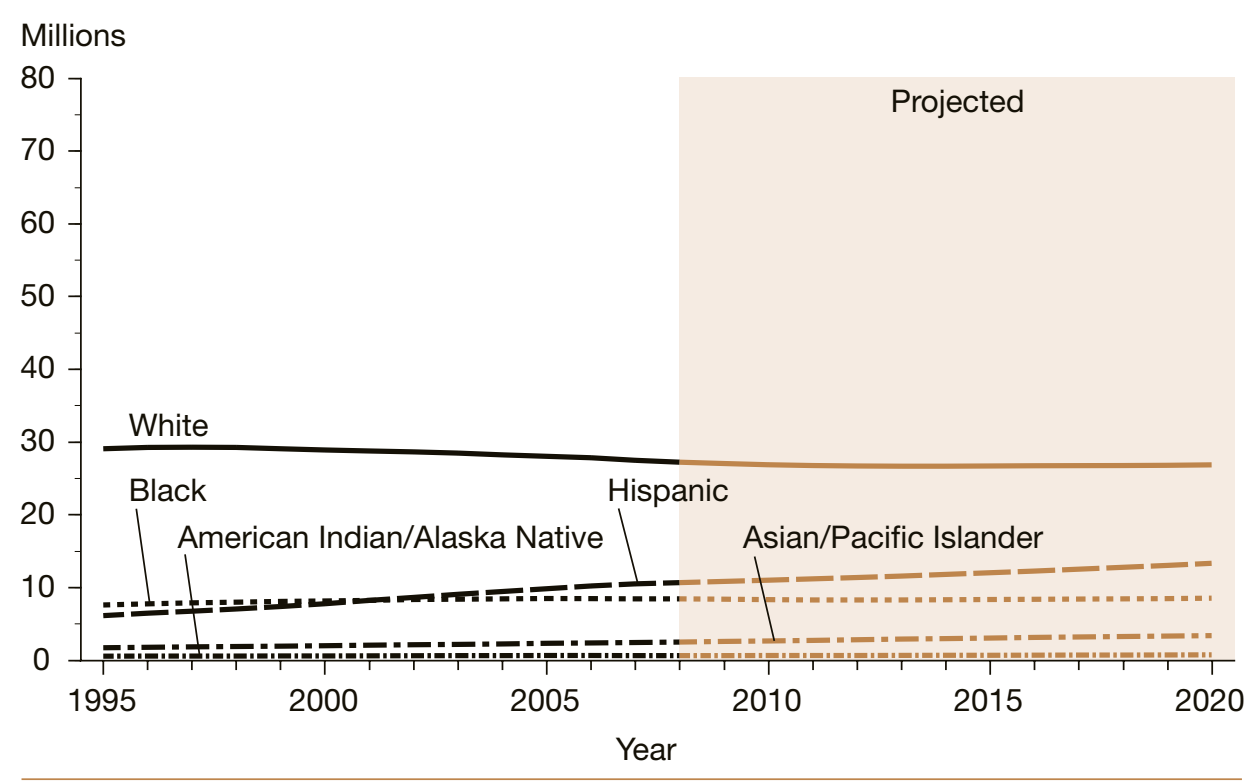

NOTE: The historical racial/ethnic time-series were constructed using racial/ethnic enrollment data at the state level for individual grades. In some instances, enrollment data by race/ethnicity had to be imputed. Further, in some instances, the racial/ethnic enrollment data for individual grades had to be adjusted to the state total for that grade. Beginning in 2008, jurisdictions could classify students as being of two or more races. As a limited number of states classified students as being of two or more races, those students were proportioned among the other categories. For additional information see the Elementary and Secondary Enrollment section A.1 in appendix A. Race categories exclude persons of Hispanic ethnicity. Mean absolute percentage errors of selected education statistics can be found in table A-2, appendix A.

SOURCE: U.S. Department of Education, National Center for Education Statistics, Common Core of Data (CCD), "State Nonfiscal Survey of Public Elementary/Secondary Education," 1995-96 through 2008-09; and National Public Elementary and Secondary Enrollment by Race/Ethnicity Model, 1994-2008. (This figure was prepared February 2011.)

\section{Enrollment by control of school}

Enrollment in public elementary and secondary schools

$\triangle$ increased 10 percent between 1995 and 2008; and

$\Delta$ is projected to increase an additional 7 percent between 2008 and 2020.

Enrollment in private elementary and secondary schools

$\nabla$ decreased 4 percent between 1995 and 2008; and

$\nabla$ is projected to decrease 8 percent between 2008 and 2020 .

For more information:

Table 1

\section{Enrollment by race/ ethnicity}

Between 2008 and 2020, enrollment in public elementary and secondary schools is projected to

$\nabla$ decrease 1 percent for students who are White;

$\Delta$ increase 1 percent for students who are Black;

$\Delta$ increase 25 percent for students who are Hispanic;

- increase 36 percent for students who are Asian/Pacific Islander; and

$\Delta$ increase 17 percent for students who are American Indian/Alaska Native.

For more information:

Tables 3, 4, and 5 


\section{Enrollment by state}

The expected 7 percent national increase in public school enrollment between 2008 and 2020 plays out differently among the states.

$\Delta \quad$ Increases are projected for 36 states and the District of Columbia, with

- increases of more than 15 percent projected for 8 states;

- increases between 5 and 15 percent projected for 17 states and the District of Columbia; and

- increases of less than 5 percent projected for 11 states.

$\nabla$ Decreases are projected for 14 states, with

- decreases of 5 percent or more projected for 3 states; and

- decreases of less than 5 percent projected for 11 states.

For more information:

Tables 6 through 11

\section{Enrollment by region}

Between 2008 and 2020, public elementary and secondary enrollment is projected to

$\nabla$ decrease 3 percent in the Northeast;

$\triangle$ increase 1 percent in the Midwest;

$\Delta$ increase 10 percent in the South; and

$\Delta$ increase 13 percent in the West.

\section{For more information:}

Tables 6 through 11
Figure 4. Projected percentage change in enrollment in public elementary and secondary schools, by state: Fall 2008 through fall 2020

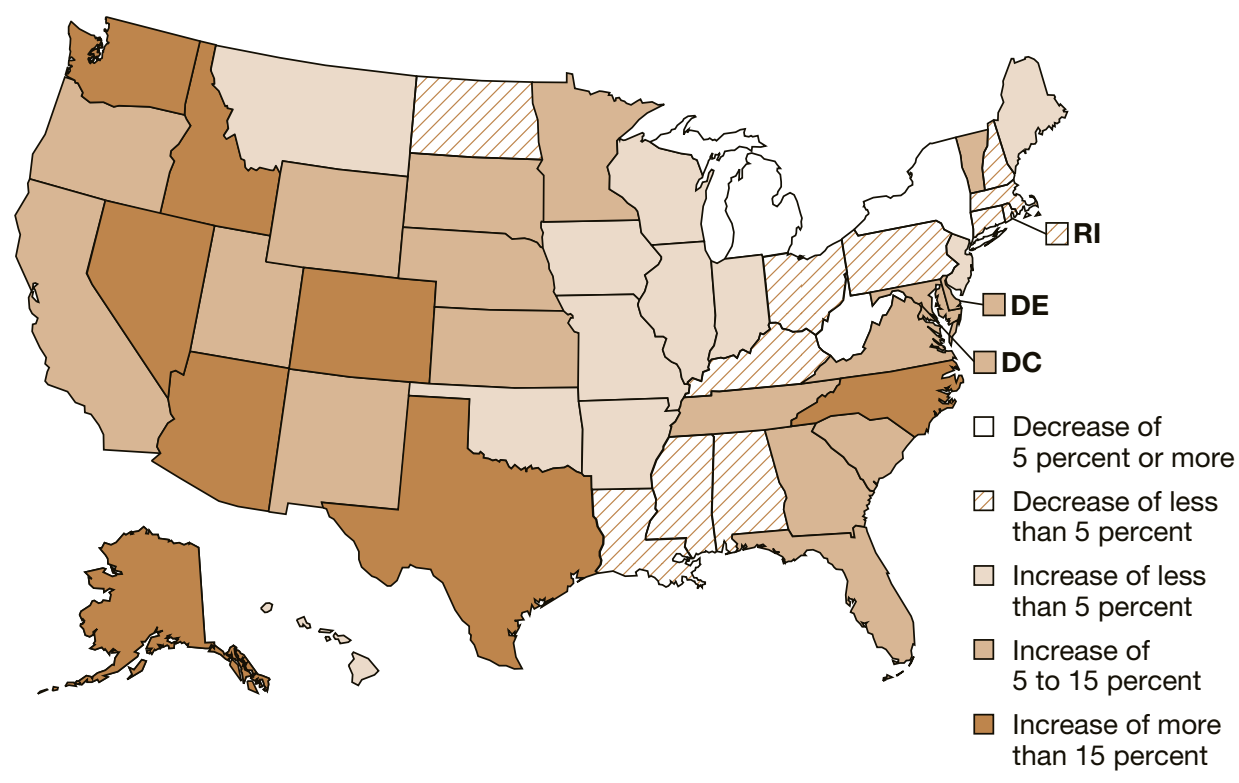

NOTE: Calculations are based on unrounded numbers. Mean absolute percentage errors of enrollment in public elementary and secondary schools by state and region can be found in table A-7, appendix A.

SOURCE: U.S. Department of Education, National Center for Education Statistics, Common Core of Data (CCD), "State Nonfiscal Survey of Public Elementary/Secondary Education," 2008-09; and State Elementary and Secondary Enrollment Model, 1980-2008. (This figure was prepared February 2011.)

Figure 5. Actual and projected numbers for enrollment in public elementary and secondary schools, by region: Fall 2002, fall 2008, and fall 2020

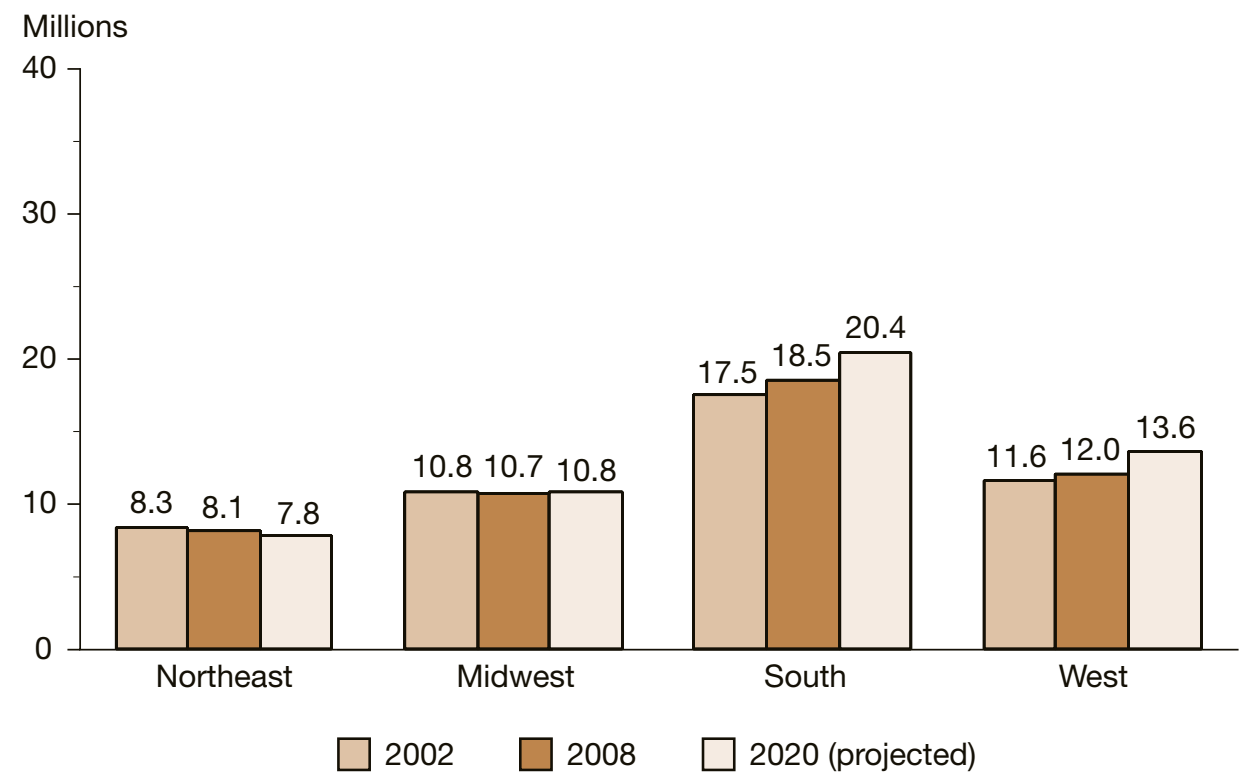

NOTE: Calculations are based on unrounded numbers. See the glossary for a list of the states in each region. Mean absolute percentage errors of enrollment in public elementary and secondary schools by state and region can be found in table A-7, appendix A.

SOURCE: U.S. Department of Education, National Center for Education Statistics, Common Core of Data (CCD), "State Nonfiscal Survey of Public Elementary/Secondary Education," 2002-03 and 2008-09; and State Elementary and Secondary Enrollment Model, 1980-2008. (This figure was prepared February 2011.) 


\section{Section 2 High School Graduates}

\section{INTRODUCTION}

The number of high school graduates increased nationally by 32 percent between 1995-96 and 2007-08, the last year of actual data. A decrease of 3 percent is expected between 2007-08 and 2020-21. Both public schools and private schools are expected to have decreases in the number of high school graduates. Increases are expected in the West and South, and decreases are expected in the Northeast and Midwest.

\section{Factors affecting the projections}

The projections of high school graduates are related to projections of 12th-graders and the historical relationship between the number of 12th-graders and the number of high school graduates. The methodology implicitly includes the net effect of factors such as dropouts, transfers to and from public schools, and, at the state level, migration. For more details, see appendixes A.0 and A.2.

\section{About high school graduates}

A high school graduate is defined as an individual who has received formal recognition from school authorities, by the granting of a diploma, for completing a prescribed course of study. This definition does not include other high school completers or high school equivalency recipients. Projected changes in the number of graduates reflect changes in the 18-year-old population over the projection period, rather than projected changes in the graduation rates of 12 thgraders. Projections of graduates could be affected by changes in policies influencing graduation requirements.

\section{Accuracy of Projections}

For National Center for Education Statistics (NCES) projections of public high school graduates produced over the last 20 years, the mean absolute percentage errors (MAPEs) for lead times of 1, 2, 5, and 10 years out were 1.0, 1.0, 1.6, and 4.0, respectively. For NCES projections of private high school graduates produced over the last 9 years, the MAPEs for lead times of $1,2,5$, and 10 years out were $0.9,0.9,5.0$, and 1.6, respectively. For more information, see table A-2 in appendix A. 


\section{NATIONAL}

The total number of high school graduates

$\triangle \quad$ increased 32 percent between 1995-96 and 2007-08, a period of 12 years; and

$\nabla$ is projected to decrease 3 percent between 2007-08 and 2020-21, a period of 13 years.

The number of public high school graduates

$\Delta$ increased 32 percent between 1995-96 and 2007-08; and

$\nabla$ is projected to decrease 1 percent between 2007-08 and 2020-21.

The number of private high school graduates

$\Delta \quad$ increased 27 percent between 1995-96 and 2007-08; and

$\nabla \quad$ is projected to decrease 27 percent between 2007-08 and 2020-21.

\section{For more information:}

Table 12
Figure 6. Actual and projected numbers for high school graduates, by control of school: School years 1995-96 through 2020-21

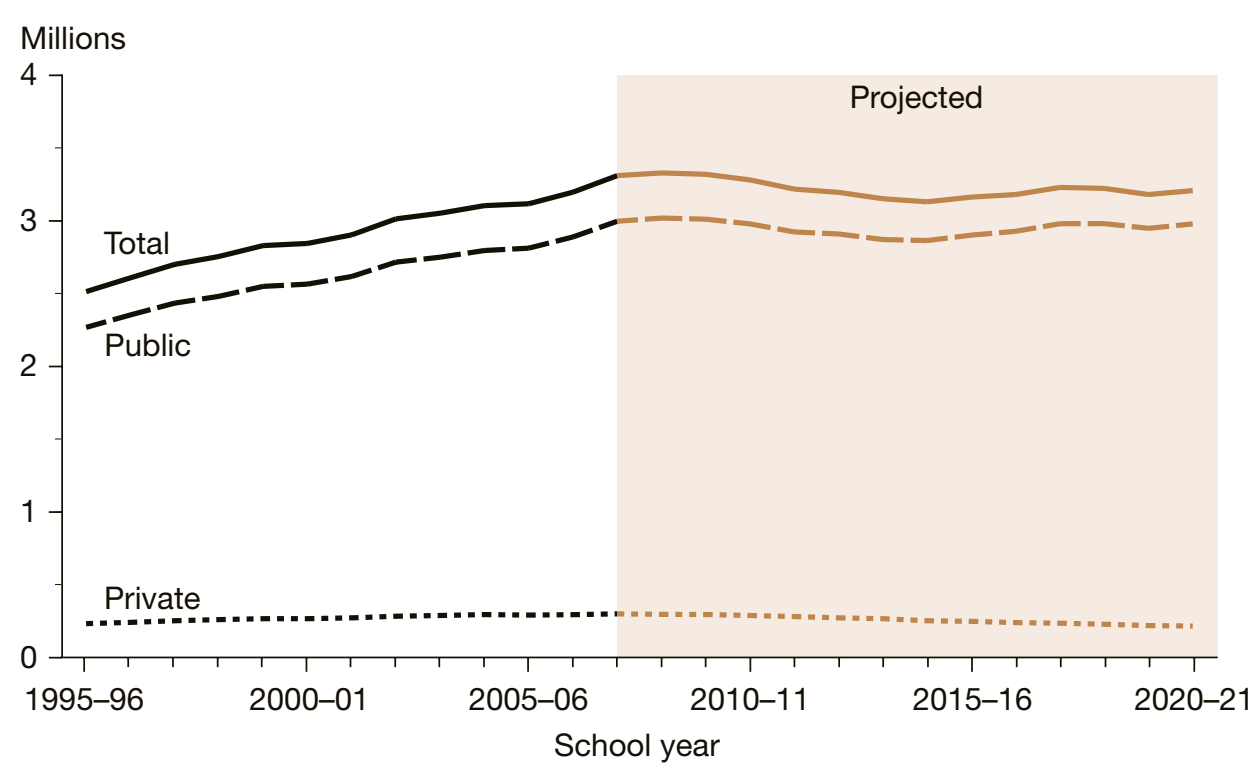

NOTE: Since the biennial Private School Universe Survey (PSS) is collected in the fall of odd numbered years, private school numbers for alternate years are estimated based on data from the PSS. Some data have been revised from previously published figures. Mean absolute percentage errors of selected education statistics can be found in table A-2, appendix A.

SOURCE: U.S. Department of Education, National Center for Education Statistics, Common Core of Data (CCD), "State Nonfiscal Survey of Public Elementary/Secondary Education," 1995-96 through 2008-09; Private School Universe Survey (PSS), selected years, 199596 through 2009-10; and National Elementary and Secondary High School Graduates Enrollment Model, 1972-73 through 2007-08. (This figure was prepared February 2011.) 
Figure 7. Actual and projected numbers for public high school graduates, by race/ethnicity: School years 1995-96 through 2020-21

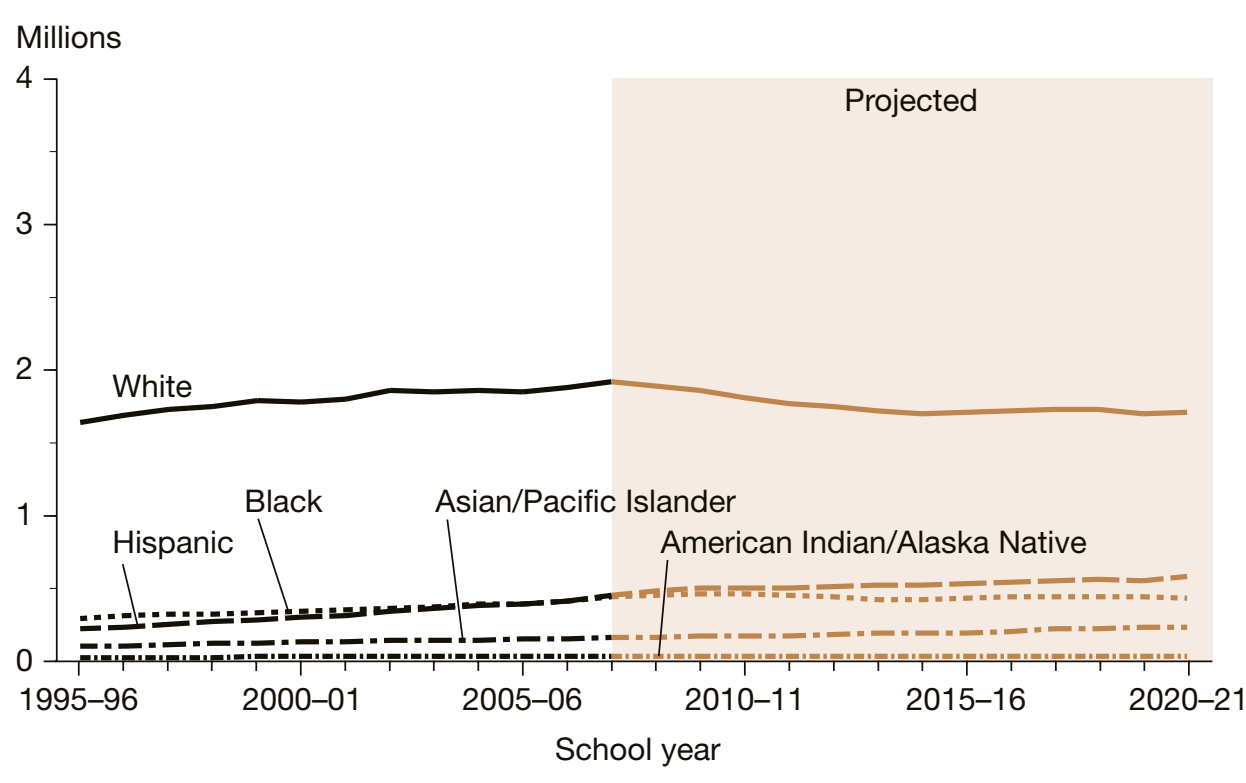

NOTE: The historical racial/ethnic time series were constructed using racial/ethnic high school graduate data at the state level. In some instances, high school graduate data by race/ethnicity had to be imputed. Further, in some instances, the racial/ethnic data had to be adjusted in order for them to sum to the state total for high school graduates. For additional information, see the High School Graduates section A.2 in appendix A. Race categories exclude persons of Hispanic ethnicity. Some data have been revised from previously published figures. Mean absolute percentage errors of selected education statistics can be found in table A-2, appendix A. SOURCE: U.S. Department of Education, National Center for Education Statistics, Common Core of Data (CCD), "State Nonfiscal Survey of Public Elementary/Secondary Education," 1996-97 through 2008-09; and National Public Elementary and Secondary High School Graduates by Race/Ethnicity Model, 1995-96 through 2007-08. (This figure was prepared February 2011.)

\section{High school graduates by race/ethnicity}

Between 2007-08 and 2020-21, the number of public high school graduates is projected to

$\nabla$ decrease 11 percent for students who are White;

$\nabla$ decrease 2 percent for students who are Black;

$\Delta \quad$ increase 27 percent for students who are Hispanic;

A increase 46 percent for students who are Asian/Pacific Islander; and

$\Delta \quad$ increase 1 percent for students who are American Indian/Alaska Native.

For more information:

Table 13 


\section{High school graduates by}

\section{state}

The expected 1 percent national decrease in public high school graduates between $2007-08$ and 2020-21 plays out differently among the states.

$\Delta \quad$ Increases are projected for 23 states, with

- increases of more than 15 percent projected for 8 states;

- increases between 5 and 15 percent projected for 8 states; and

- increases of less than 5 percent projected for 7 states.

$\checkmark$ Decreases are projected for 27 states and the District of Columbia, with

- decreases of 5 percent or more projected for 16 states and the District of Columbia; and

- decreases of less than 5 percent projected for 11 states.

For more information:

Tables 14 and 15

\section{High school graduates by region}

Between 2007-08 and 2020-21, the number of public high school graduates is projected to

$\nabla$ decrease 13 percent in the Northeast;

$\nabla$ decrease 6 percent in the Midwest;

$\Delta \quad$ increase 7 percent in the South; and

$\Delta$ increase 4 percent in the West.

For more information:

Tables 14 and 15
Figure 8. Projected percentage change in the number of public high school graduates, by state: School years 2007-08 through 2020-21

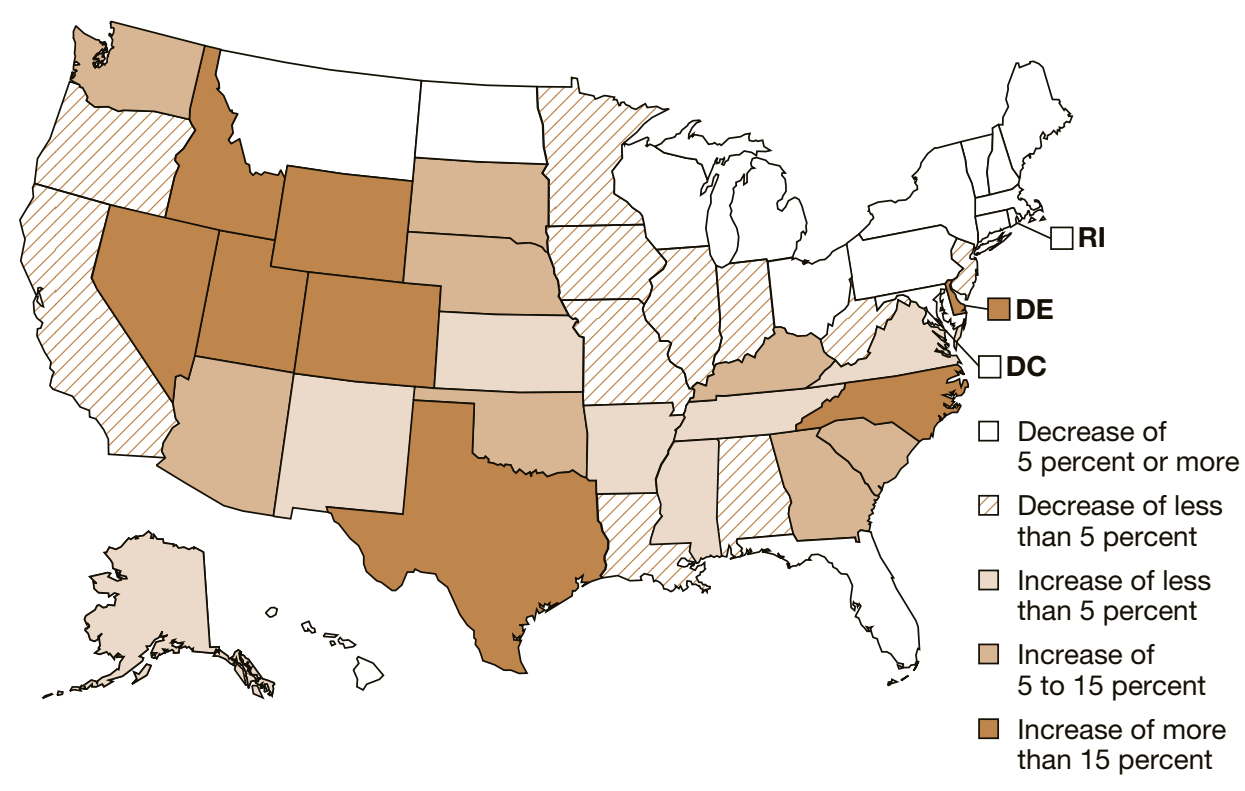

NOTE: Calculations are based on unrounded numbers. Mean absolute percentage errors of public high school graduates by state and region can be found in table A-10, appendix A.

SOURCE: U.S. Department of Education, National Center for Education Statistics, Common Core of Data (CCD), "State Nonfiscal Survey of Public Elementary/Secondary Education," 2008-09; and State Public High School Graduates Model, 1980-81 through 2007-08. (This figure was prepared February 2011.)

Figure 9. Actual and projected numbers for public high school graduates, by region: School years 2002-03, 2007-08, and 2020-21

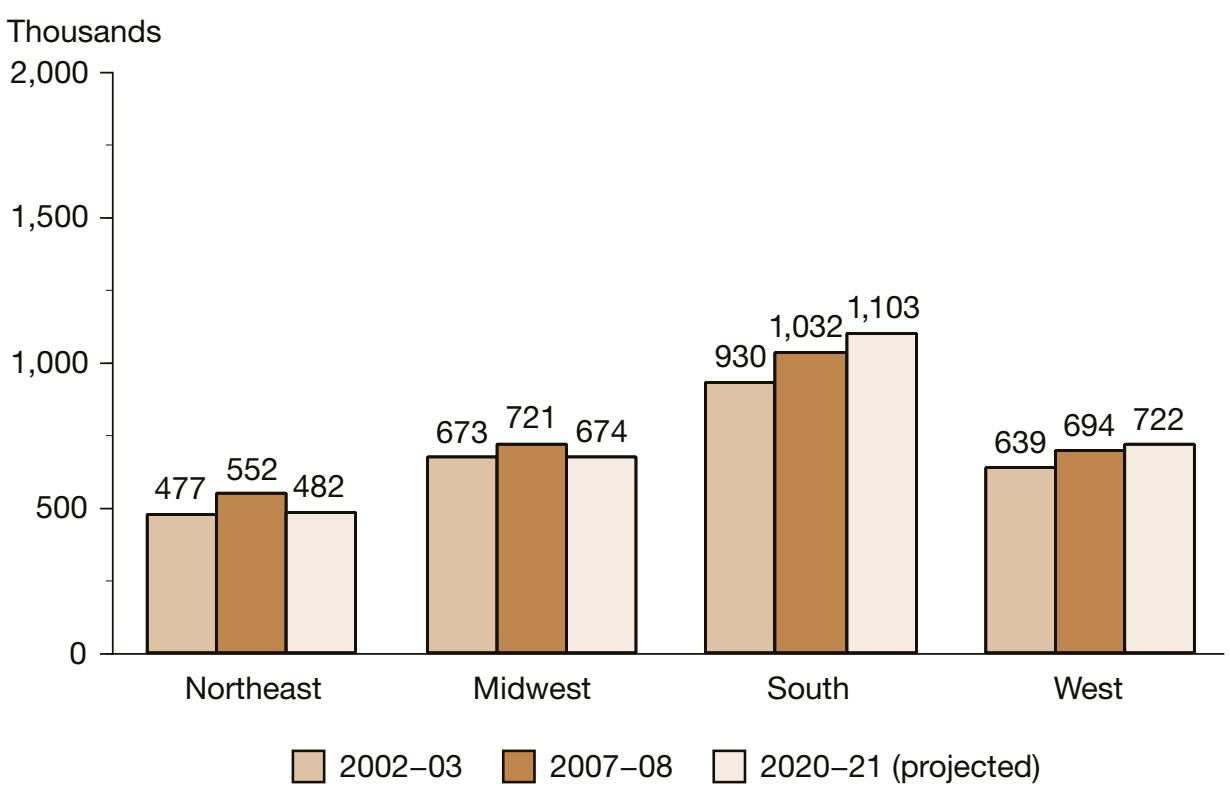

NOTE: See the glossary for a list of states in each region. Mean absolute percentage errors of public high school graduates by state and region can be found in table A-10, appendix A. SOURCE: U.S. Department of Education, National Center for Education Statistics, Common Core of Data (CCD), "State Nonfiscal Survey of Public Elementary/Secondary Education," 2003-04 and 2008-09; and State Public High School Graduates Model, 1980-81 through 2007-08. (This figure was prepared February 2011.) 


\section{Section 3 \\ Elementary and Secondary Teachers}

\section{INTRODUCTION}

Between fall 2008, the last year of actual public school data, and fall 2020, the number of teachers in elementary and secondary schools is projected to rise. The number of public school teachers is projected to increase and the number of private school teachers is projected to decrease. The pupil/teacher ratios are projected to decrease in both public and private schools. The annual number of new teacher hires is projected to increase in both public and private schools.

\section{Factors affecting the projections}

The projections of the number of elementary and secondary teachers are related to projected levels of enrollments and education revenue receipts from state sources per capita. For more details, see appendixes A. 0 and A.3.

\section{Factors that were not considered}

The projections do not take into account possible changes in the number of teachers due to the effects of government policies.

\section{About pupil/teacher ratios}

The overall elementary and secondary pupil/teacher ratio and pupil/teacher ratios for public and private schools were computed based on elementary and secondary enrollment and the number of classroom teachers by control of school.

\section{About new teacher hires}

A teacher is considered to be a new teacher hire for a certain control of school (public or private) for a given year if the teacher teaches in that control that year but had not taught in that control in the previous year. A teacher who moves from teaching in one control to the other control is considered a new teacher hire, but a teacher who moves from one school to another school in the same control is not considered a new teacher hire. It is important to note that these projections measure the total number of teacher hires, including those hired to replace teachers retiring or leaving the teaching profession permanently or temporarily. Thus, the new teacher hire projections should not be interpreted as predicting teacher shortages.

\section{Accuracy of Projections}

An analysis of projection errors from the past 20 editions of Projections of Education Statistics indicates that the mean absolute percentage errors (MAPEs) for projections of classroom teachers in public elementary and secondary schools were 1.0 percent for 1 year out, 1.4 percent for 2 years out, 2.9 percent for 5 years out, and 5.9 percent for 10 years out. For more information on the MAPEs of different National Center for Education Statistics (NCES) projection series, see table A-2 in appendix A. 


\section{Number of teachers}

The total number of elementary and secondary teachers

$\triangle$ increased 23 percent between 1995 and 2008, a period of 13 years; and

$\Delta$ is projected to increase 7 percent between 2008 and 2020, a period of 12 years.

The number of teachers in public elementary and secondary schools

$\triangle$ increased 24 percent between 1995 and 2008; and

$\Delta$ is projected to increase 9 percent between 2008 and 2020.

The number of teachers in private elementary and secondary schools

$\triangle$ increased 19 percent between 1995 and 2008; and

$\nabla$ is projected to decrease 4 percent between 2008 and 2020.

For more information:

Table 16
Figure 10. Actual and projected numbers for elementary and secondary teachers, by control of school: Fall 1995 through fall 2020

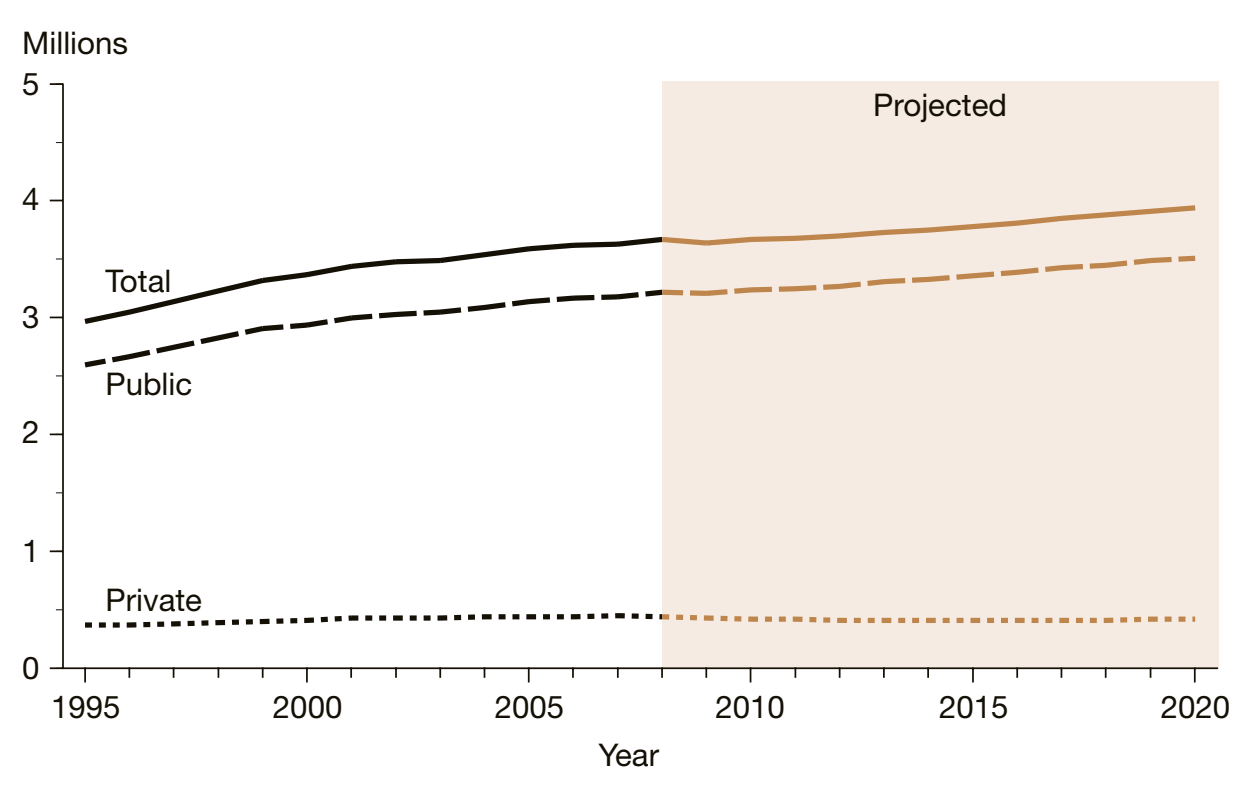

NOTE: Since the biennial Private School Universe Survey (PSS) is collected in the fall of odd numbered years, private school numbers for alternate years are estimated based on data from the PSS. The private school teacher number for 2009 is an actual number from the 2009-10 PSS. Teachers reported in full-time equivalents. Some data have been revised from previously published figures. Mean absolute percentage errors of selected education statistics can be found in table A-2, appendix A.

SOURCE: U.S. Department of Education, National Center for Education Statistics, Common Core of Data (CCD), "State Nonfiscal Survey of Public Elementary/Secondary Education," 1995-96 through 2008-09; Private School Universe Survey (PSS), selected years, 1995-96 through 2009-10; Elementary and Secondary Teacher Model, 1973-2009. (This figure was prepared April 2011.) 
Figure 11. Actual and projected numbers for the pupil/teacher ratios in elementary and secondary schools, by control of school: Fall 1995 through fall 2020

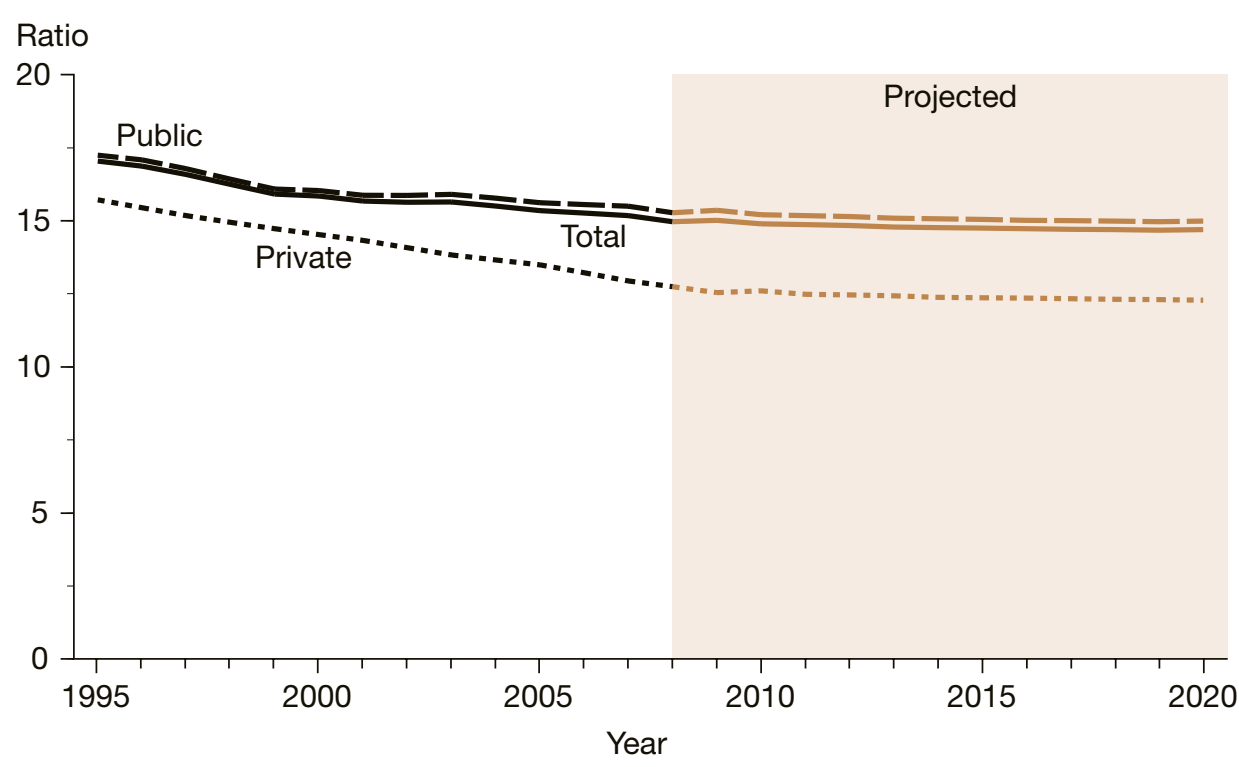

NOTE: Since the biennial Private School Universe Survey (PSS) is collected in the fall of odd numbered years, private school numbers for alternate years are estimated based on data from the PSS. The private school teacher number for 2009 is an actual number from the 2009-10 PSS. The pupil/teacher ratios were derived from tables 1 and 16. Teachers are reported in full-time equivalents. Some data have been revised from previously published figures. Mean absolute percentage errors of selected education statistics can be found in table A-2, appendix A.

SOURCE: U.S. Department of Education, National Center for Education Statistics, Common Core of Data (CCD), "State Nonfiscal Survey of Public Elementary/Secondary Education," 1995-96 through 2008-09; Private School Universe Survey (PSS), selected years, 1995-96 through 2009-10; National Elementary and Secondary Enrollment Model, 1972-2008; and Elementary and Secondary Teacher Model, 1973-2009. (This figure was prepared April 2011.)

Figure 12. Actual and projected numbers for elementary and secondary new teacher hires, by control of school: Fall 1999, fall 2008, and fall 2020

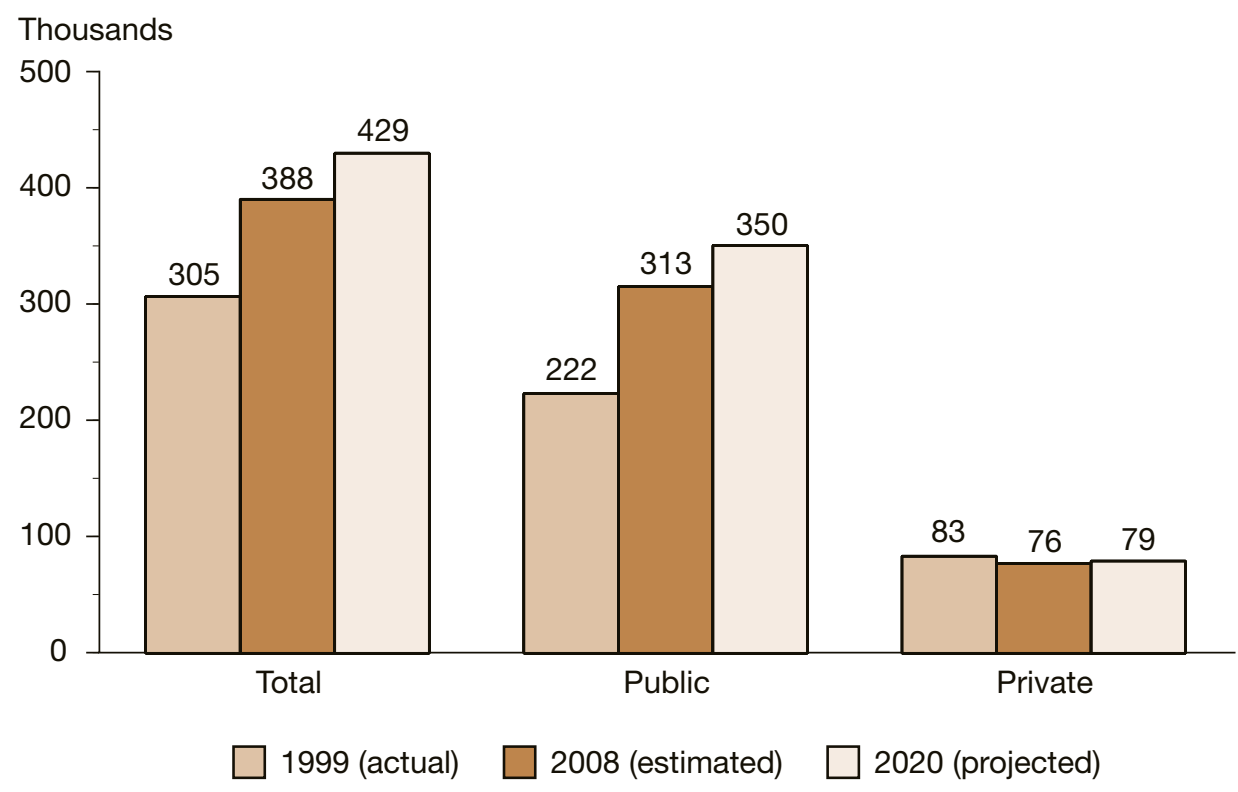

NOTE: Public and private new teacher hire numbers for 2008 are estimated using the New Teacher Hires Model.

SOURCE: U.S. Department of Education, National Center for Education Statistics, Common Core of Data (CCD), "State Nonfiscal Survey of Public Elementary/Secondary Education," 1999-2000; Private School Universe Survey (PSS), 1999-2000; Schools and Staffing Survey (SASS), "Public

School Teacher Questionnaire," 1999-2000 and "Private School Teacher Questionnaire," 1999-2000; Elementary and Secondary Teacher Model, 1973-2009; and New Teacher Hires Model, 1988-2007. (This figure was prepared April 2011.)

\section{Pupil/teacher ratios}

The pupil/teacher ratio in elementary and secondary schools

$\nabla$ decreased from 17.1 to 15.0 between 1995 and 2008; and

$\nabla$ is projected to decrease to 14.7 in 2020 .

The pupil/teacher ratio in public elementary and secondary schools

$\nabla$ decreased from 17.3 to 15.3 between 1995 and 2008; and

$\nabla$ is projected to decrease to 15.0 in 2020.

The pupil/teacher ratio in private elementary and secondary schools

$\nabla$ decreased from 15.7 to 12.8 between 1995 and 2008; and

$\nabla$ is projected to decrease to 12.3 in 2020 .

For more information:

Table 17

\section{New teacher hires}

Between 2008 and 2020, increases are expected in the annual numbers of new public school teacher hires and new private school teacher hires.

The number of new teacher hires in public schools

- was approximately 313,000 in 2008, reflecting an increase of 41 percent compared to 1999 ; and

$\Delta$ is projected to increase 12 percent to 350,000 in 2020.

The number of new teacher hires in private schools

- was approximately 76,000 in 2008 , reflecting a decrease of 9 percent compared to 1999 ; and

$\Delta$ is projected to increase 5 percent to 79,000 in 2020 .

For more information:

Table 16 
This page intentionally left blank. 


\section{Section 4 \\ Expenditures for Public Elementary and Secondary Education}

\section{INTRODUCTION}

Current expenditures for public elementary and secondary education are projected to increase 22 percent in constant dollars between school years 2007-08, the last year of actual data, and 2020-21.

\section{Factors affecting the projections}

The projections of current expenditures are related to projections of economic growth as measured by disposable income per capita and assistance by state governments to local governments. For more details, see appendixes A.0 and A.4.

\section{Factors that were not considered}

Many factors that may affect future school expenditures were not considered in the production of these projections. Such factors include policy initiatives as well as potential changes in the age distribution of elementary and secondary teachers as older teachers retire and are replaced by younger teachers.

\section{About constant dollars and current dollars}

Throughout this section, projections of current expenditures are presented in constant 2008-09 dollars. The reference tables, later in this report, present these data both in constant 2008-09 dollars and in current dollars. The projections were developed in constant dollars and then placed in current dollars using projections for the Consumer Price Index (CPI) (table B-6 in appendix B).

\section{Accuracy of Projections}

An analysis of projection errors from similar models used in the past 20 editions of Projections of Education Statistics that contained expenditure projections indicates that mean absolute percentage errors (MAPEs) for current expenditures in constant dollars were 1.3 percent for 1 year out, 2.0 percent for 2 years out, 2.6 percent for 5 years out, and 4.1 percent for 10 years out. MAPEs for current expenditures per pupil in fall enrollment in current dollars were 1.3 percent for 1 year out, 2.0 percent for 2 years out, 3.0 percent for 5 years out, and 5.5 percent for 10 years out. See appendix A for further discussion of the accuracy of recent projections of current expenditures, and see table A-2 in appendix A for the mean absolute percentage errors (MAPEs) of these projections. 


\section{Current expenditures}

Current expenditures in constant 2008-09 dollars

$\triangle$ increased 45 percent from 1995-96 to 2007-08, a period of 12 years; and

$\Delta$ are projected to increase 22 percent, to $\$ 627$ billion, from 2007-08 to 2020-21, a period of 13 years.

\section{For more information:}

Tables 18 and 19
Figure 13. Actual and projected current expenditures for public elementary and secondary schools (in constant 2008-09 dollars): School years 1995-96 through 2020-21

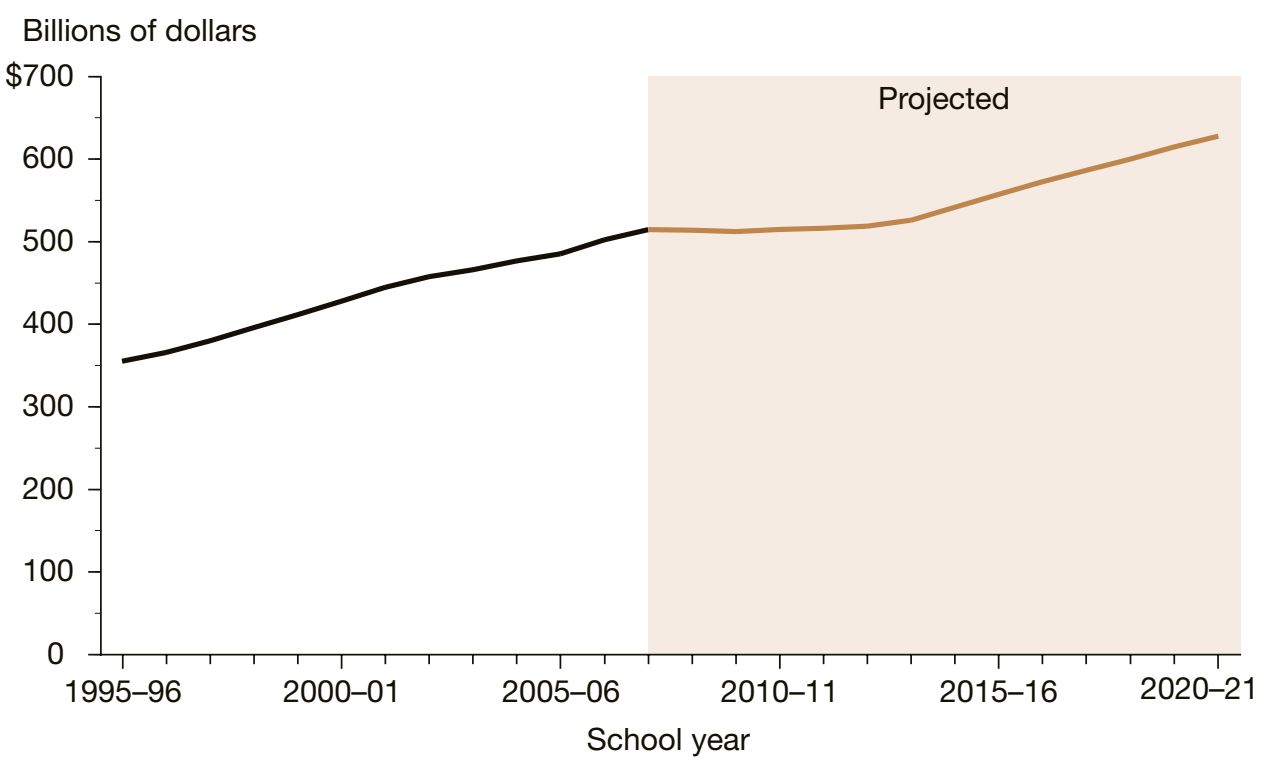

NOTE: Numbers were placed in constant dollars using the Consumer Price Index (CPI) for all urban consumers, Bureau of Labor Statistics, U.S. Department of Labor. For more detail about CPI, see table B-6 in appendix B. Some data have been revised from previously published figures. Mean absolute percentage errors of selected education statistics can be found in table A-2, appendix A.

SOURCE: U.S. Department of Education, National Center for Education Statistics, Common Core of Data (CCD), "National Public Education Financial Survey," 1995-96 through 2007-08; Public Elementary and Secondary School Current Expenditures Model, 1969-70 through 2007-08. (This figure was prepared March 2011.) 
Figure 14. Actual and projected current expenditures per pupil in fall enrollment in public elementary and secondary schools (in constant 2008-09 dollars): School years 1995-96 through 2020-21

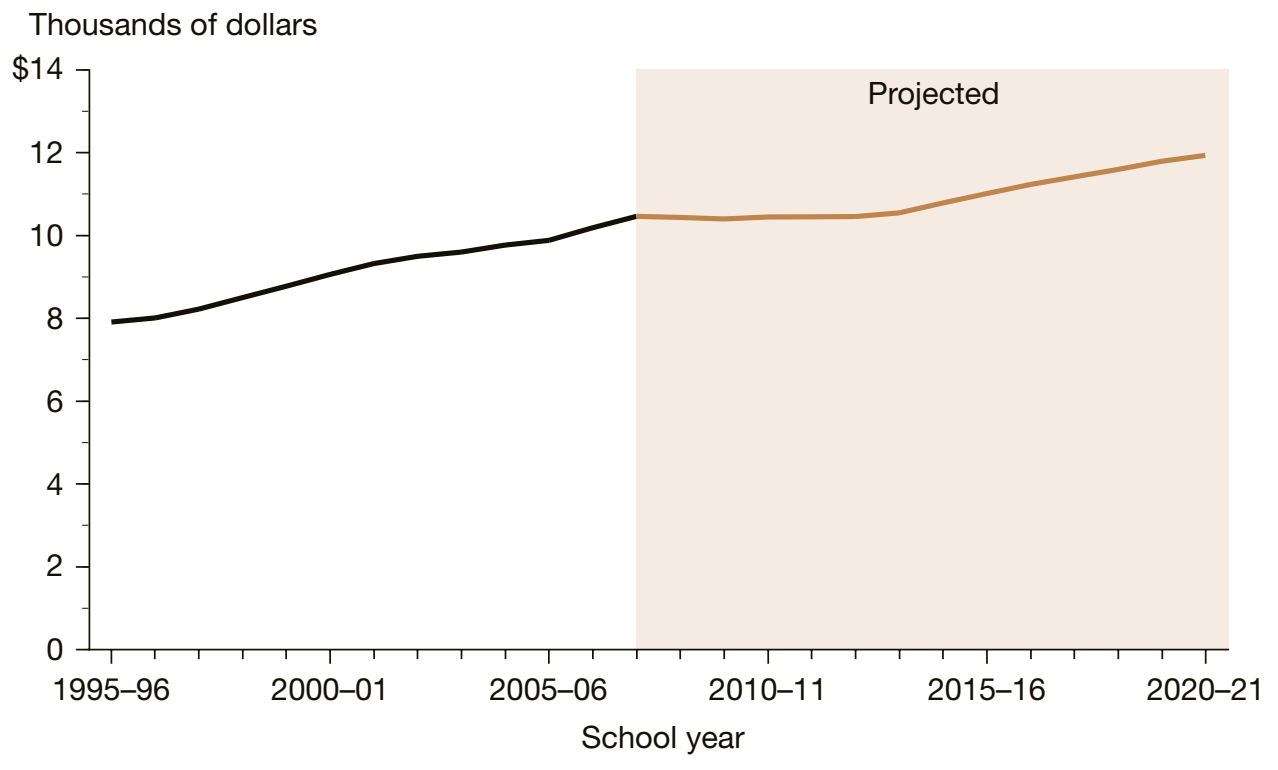

Thousands of dollars

NOTE: Numbers were placed in constant dollars using the Consumer Price Index (CPI) for all urban consumers, Bureau of Labor Statistics, U.S. Department of Labor. For more detail about CPI, see table B-6 in appendix B. Some data have been revised from previously published figures. Mean absolute percentage errors of selected education statistics can be found in table A-2, appendix A.

SOURCE: U.S. Department of Education, National Center for Education Statistics, Common Core of Data (CCD), "State Nonfiscal Survey of Public Elementary/Secondary Education," 1995-96 through 2008-09; "National Public Education Financial Survey," 1995-96 through 2007-08; National Elementary and Secondary Enrollment Model, 1972-2008; and Public Elementary and Secondary School Current Expenditures Model, 1969-70 through 2007-08. (This figure was prepared March 2011.)

\section{Current expenditures per} pupil

Current expenditures per pupil in fall enrollment in constant 2008-09 dollars

$\Delta$ increased 32 percent from $1995-96$ to $2007-08$; and

$\Delta$ are projected to increase 14 percent, to $\$ 11,900$, from 2007-08 to 2020-21.

For more information:

Tables 18 and 19 
This page intentionally left blank. 


\section{Section 5 \\ Enrollment in Postsecondary Degree-Granting Institutions}

\section{INTRODUCTION}

Total enrollment in postsecondary degree-granting institutions is expected to increase 13 percent between fall 2009, the last year of actual data, and fall 2020. Degree-granting institutions are postsecondary institutions that provide study beyond secondary school and offer programs terminating in an associate's, baccalaureate, or higher degree and participate in federal financial aid programs. Differential growth is expected by student characteristics such as age, sex, and attendance status (parttime or full-time). Enrollment is expected to increase in both public and private postsecondary degree-granting institutions.

\section{Factors affecting the projections}

The projections of enrollment levels are related to projections of college-age populations, disposable income, and unemployment rates. For more details, see appendixes A.0 and A.5. An important factor in the enrollment projections is the expected increase in the population of 25- to 29-yearolds (table B-4 in appendix B).

Figure 15. Actual and projected population numbers for 18- to 24-year-olds and 25- to 29-year-olds: 1995 through 2020

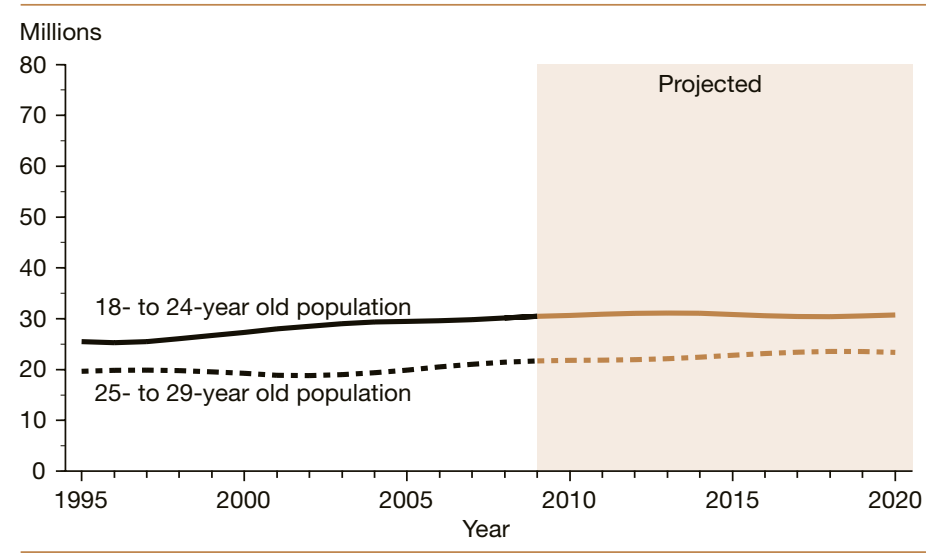

NOTE: Some data have been revised from previously published figures. Projections are from the U.S. Census Bureau's 2008 National Population Projections, ratio-adjusted to line up with the most recent historical estimate. SOURCE: U.S. Department of Commerce, Census Bureau, Population Estimates, retrieved October 4, 2010, from http://www.census.gov/popest/ national/; and Population Projections, retrieved November 2, 2009, from http://www.census.gov/population/www/projections/2008projections.html. (This figure was prepared March 2011.)

\section{Factors that were not considered}

The enrollment projections do not take into account such factors as the cost of a college education, the economic value of an education, and the impact of distance learning due to technological changes. These factors may produce changes in enrollment levels. The racial/ethnic backgrounds of nonresident aliens are not known.

\section{Accuracy of Projections}

For projections of total enrollment in postsecondary degree-granting institutions, an analysis of projection errors based on the past 13 editions of Projections of Education Statistics indicates that the mean absolute percentage errors (MAPEs) for lead times of 1, 2, 5, and 10 years out were 1.6, 2.8, 5.2, and 11.4 percent, respectively. For the 1-year-out prediction, this means that one would expect the projection to be within 1.6 percent of the actual value, on average. For more information, see table A-2 in appendix A. 


\section{TOTAL ENROLLMENT}

\section{Total enrollment in} postsecondary degreegranting institutions

A increased 43 percent from 1995 to 2009 , a period of 14 years; and

- is projected to increase 13 percent, to 23 million, from 2009 to 2020 , a period of 11 years.

For more information:

Table 20
Figure 16. Actual and projected numbers for total enrollment in all postsecondary degree-granting institutions: Fall 1995 through fall 2020

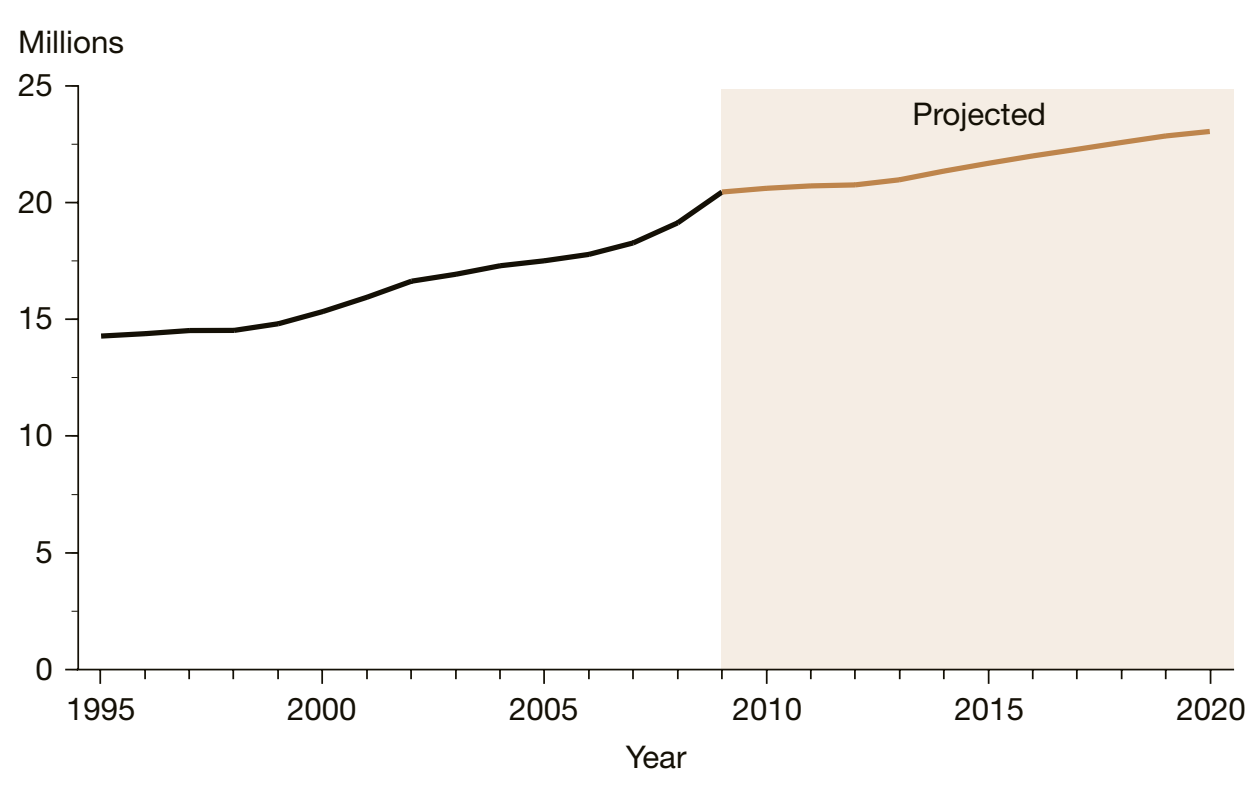

NOTE: Some data have been revised from previously published figures. Mean absolute percentage errors of selected education statistics can be found in table A-2, appendix A. SOURCE: U.S. Department of Education, National Center for Education Statistics, Integrated Postsecondary Education Data System, "Fall Enrollment Survey" (IPEDS-EF:95-99), and Spring 2001 through Spring 2010; and Enrollment in Degree-Granting Institutions Model, 1980-2009. (This figure was prepared March 2011.) 


\section{ENROLLMENT BY SELECTED CHARACTERISTICS AND CONTROL OF INSTITUTION}

Figure 17. Actual and projected numbers for enrollment in all postsecondary degreegranting institutions, by age group: Fall 1995, fall 2009, and fall 2020

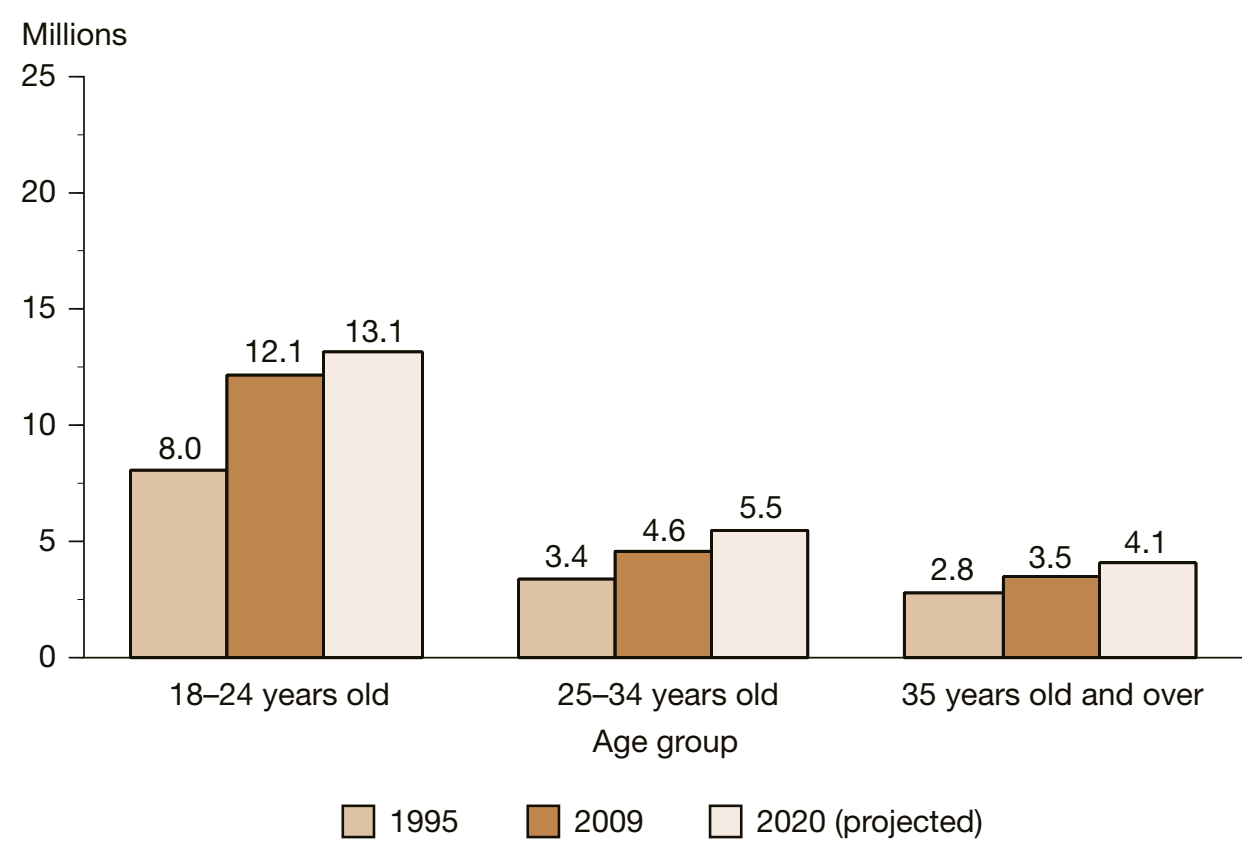

NOTE: Some data have been revised from previously published figures. Data by age are based on the distribution by age from the Census Bureau. Mean absolute percentage errors of selected education statistics can be found in table A-2, appendix A.

SOURCE: U.S. Department of Education, National Center for Education Statistics, Integrated Postsecondary Education Data System, "Fall Enrollment Survey" (IPEDS-EF:95) and Spring 2010; Enrollment in Degree-Granting Institutions Model, 1980-2009; and U.S. Department of Commerce, Census Bureau, Current Population Reports, "Social and Economic Characteristics of Students," various years. (This figure was prepared March 2011.)

Figure 18. Actual and projected numbers for enrollment in all postsecondary degree-granting institutions, by sex: Fall 1995 through fall 2020

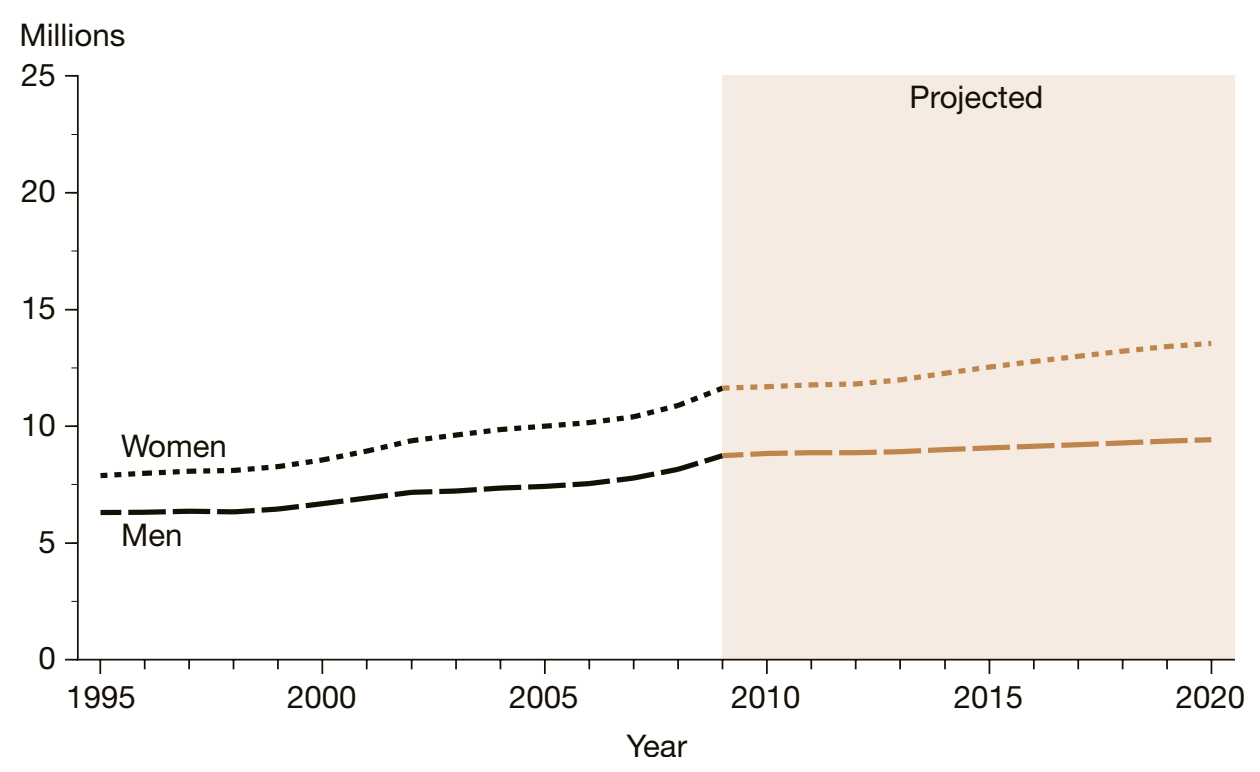

NOTE: Some data have been revised from previously published figures. Mean absolute percentage errors of selected education statistics can be found in table A-2, appendix A. SOURCE: U.S. Department of Education, National Center for Education Statistics, Integrated Postsecondary Education Data System, "Fall Enrollment Survey" (IPEDS-EF:95-99), and Spring 2001 through Spring 2010; and Enrollment in Degree-Granting Institutions Model, 1980-2009. (This figure was prepared March 2011.)

\section{Enrollment by age of student}

Between 2009 and 2020, enrollment is projected to increase

$\triangle 9$ percent for students who are 18 to 24 years old;

- 21 percent for students who are 25 to 34 years old; and

$\triangle 16$ percent for students who are 35 years old and over.

For more information:

Table 21

\section{Enrollment by sex of student}

Between 2009 and 2020, enrollment is projected to increase

A 8 percent for men; and

$\Delta \quad 16$ percent for women.

For more information:

Tables 20-26 


\section{Enrollment by attendance status}

Between 2009 and 2020, enrollment is projected to increase

$\Delta 11$ percent for full-time students; and

$\Delta \quad 16$ percent for part-time students.

For more information:

Tables 20-27

\section{Enrollment by level of student}

Between 2009 and 2020, enrollment is projected to increase

$\triangle 12$ percent for undergraduate students; and

- 18 percent for postbaccalaureate students.

For more information:

Tables 27-28
Figure 19. Actual and projected numbers for enrollment in all postsecondary degreegranting institutions, by attendance status: Fall 1995 through fall 2020

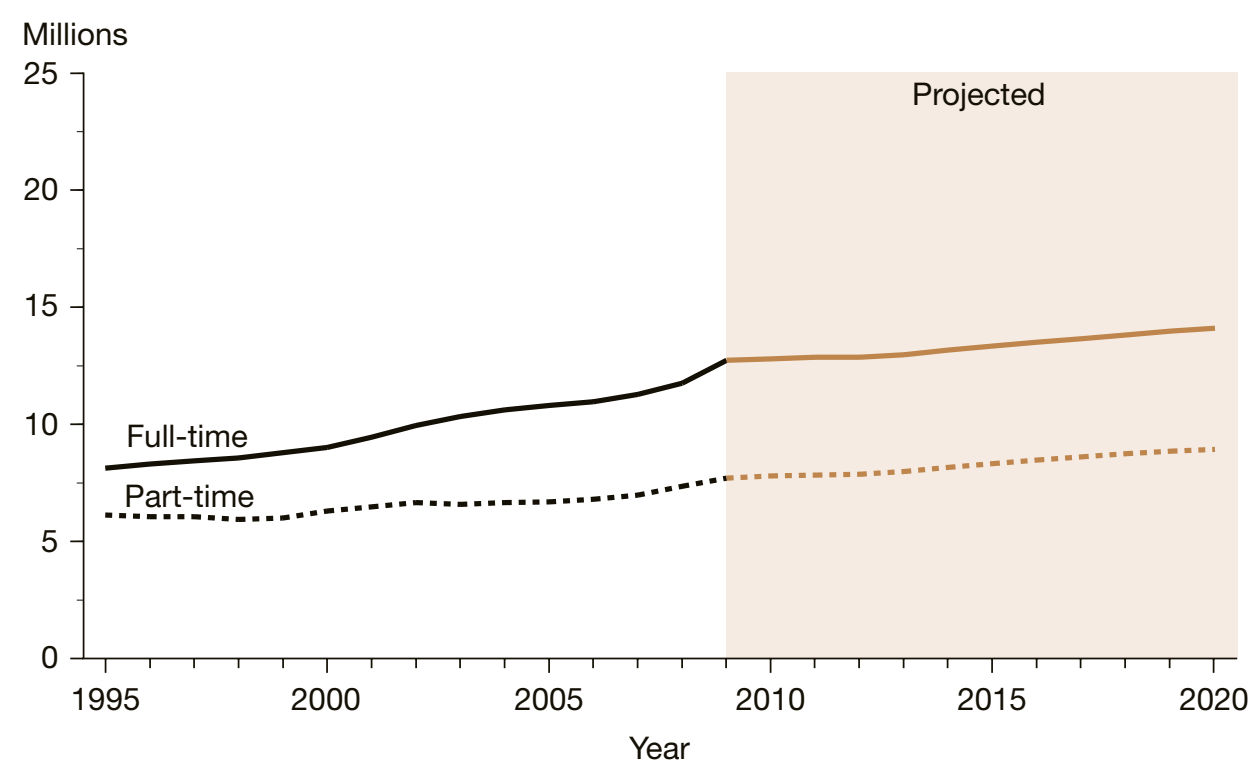

NOTE: Some data have been revised from previously published figures. Mean absolute percentage errors of selected education statistics can be found in table A-2, appendix A. SOURCE: U.S. Department of Education, National Center for Education Statistics, Integrated Postsecondary Education Data System, "Fall Enrollment Survey" (IPEDS-EF:95-99), and Spring 2001 through Spring 2010; and Enrollment in Degree-Granting Institutions Model, 1980-2009. (This figure was prepared March 2011.)

Figure 20. Actual and projected numbers for undergraduate and postbaccalaureate enrollment in all postsecondary degree-granting institutions: Fall 1995 through fall 2020

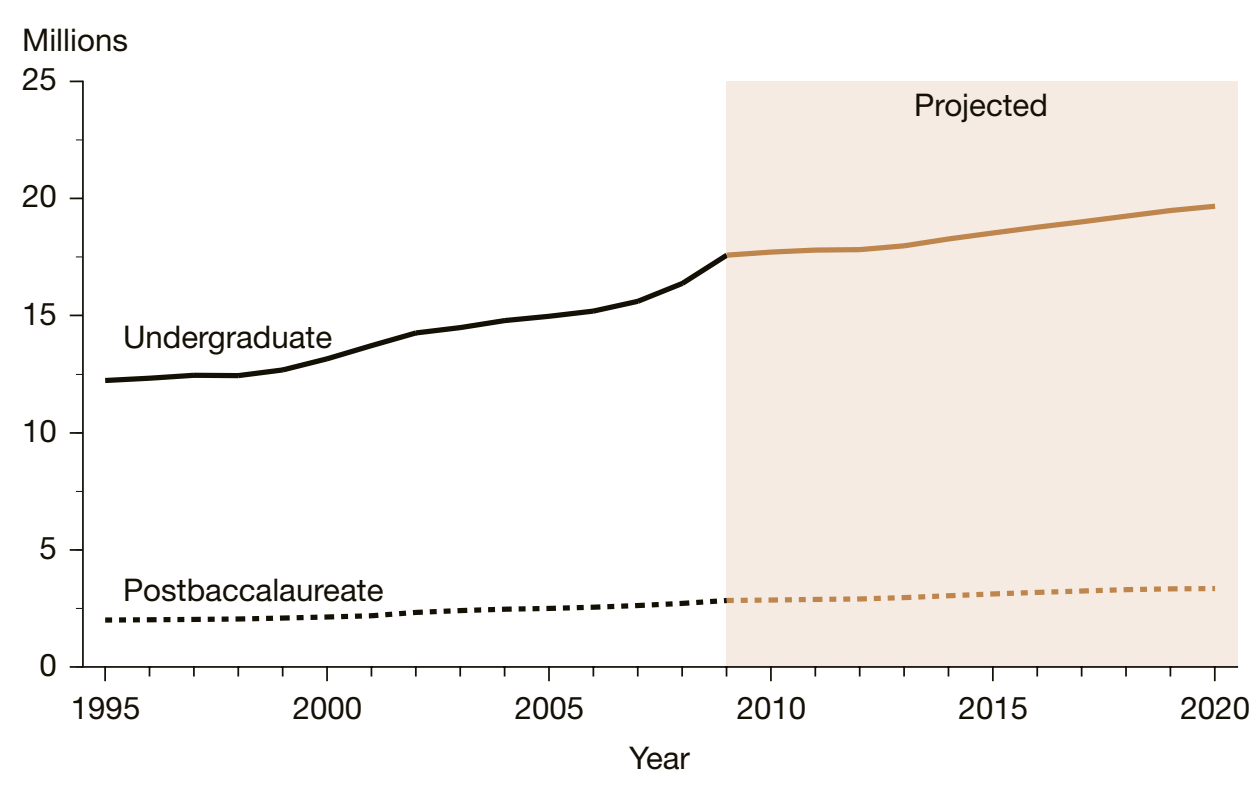

NOTE: Some data have been revised from previously published figures. Mean absolute percentage errors of selected education statistics can be found in table A-2, appendix A. SOURCE: U.S. Department of Education, National Center for Education Statistics, Integrated Postsecondary Education Data System, "Fall Enrollment Survey" (IPEDS-EF:95-99), and Spring 2001 through Spring 2010; and Enrollment in Degree-Granting Institutions Model, 1980-2009. (This figure was prepared April 2011.) 
Figure 21. Actual and projected numbers for enrollment in all postsecondary degree-granting institutions, by race/ethnicity: Fall 1995 through fall 2020

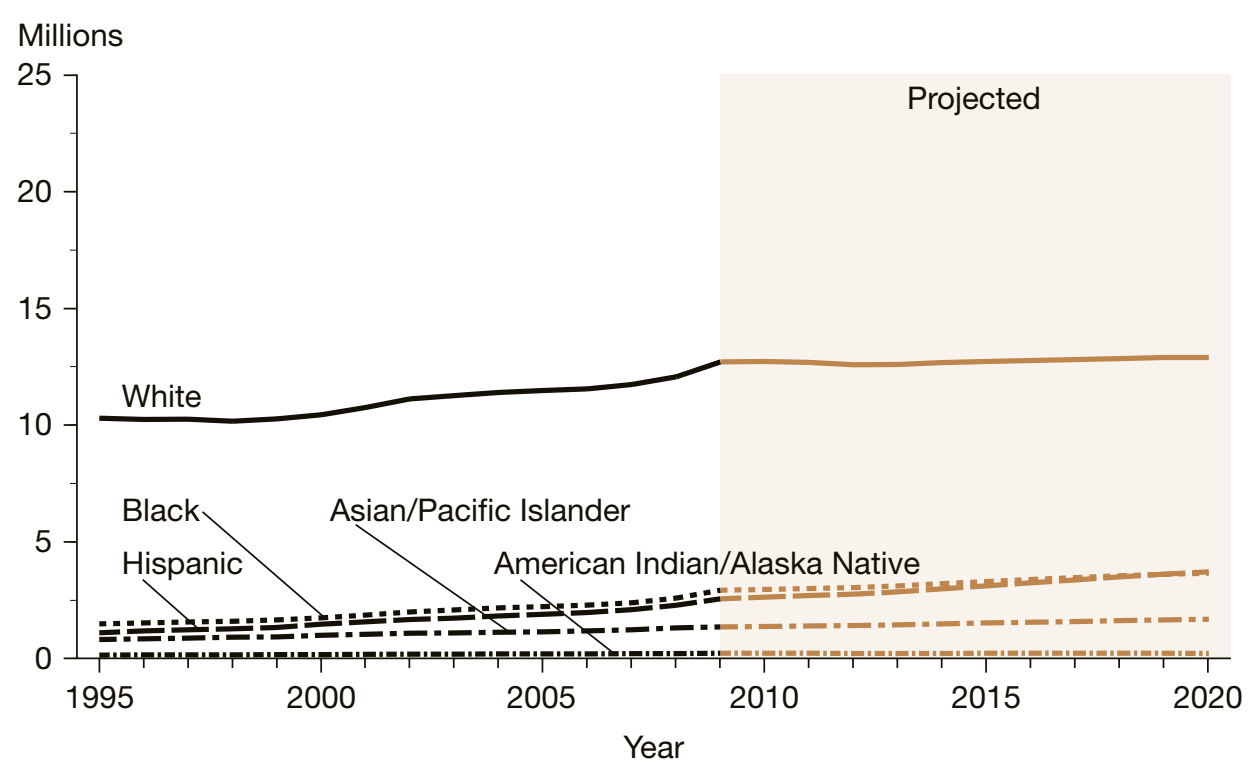

NOTE: Race categories exclude persons of Hispanic ethnicity. Enrollment data in the "race/ethnicity unknown" (all years) and "two or more races" (2008 and 2009 only) categories of the IPEDS "Fall Enrollment Survey" have been prorated to the other racial/ethnic categories at the institutional level. Mean absolute percentage errors of selected education statistics can be found in table A-2, appendix A. SOURCE: U.S. Department of Education, National Center for Education Statistics, Integrated Postsecondary Education Data System, "Fall Enrollment Survey" (IPEDS-EF:95-99), and Spring 2001 through Spring 2010; and Enrollment in Degree-Granting Institutions by Race/Ethnicity Model, 19802009. (This figure was prepared March 2011.)

Figure 22. Actual and projected numbers for enrollment in all postsecondary degree-granting institutions, by control of institution: Fall 1995 through fall 2020

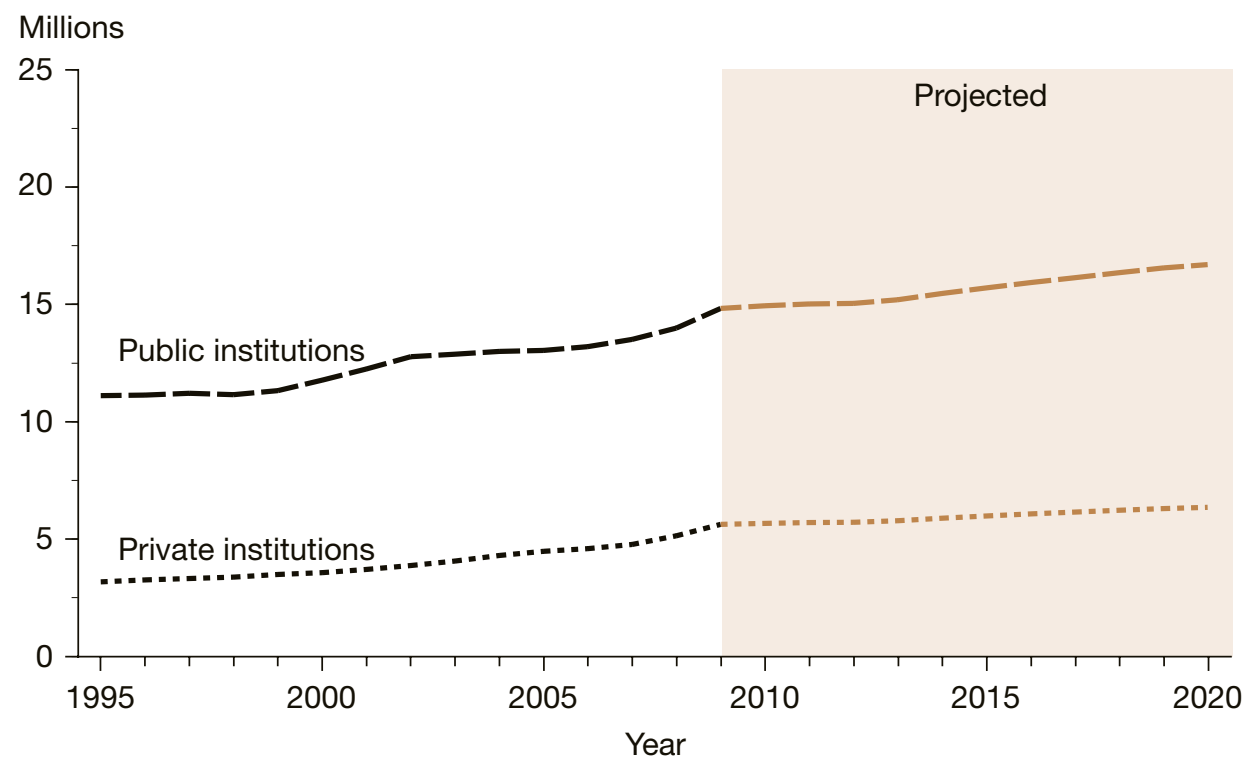

NOTE: Some data have been revised from previously published figures. Mean absolute percentage errors of selected education statistics can be found in table A-2, appendix A. SOURCE: U.S. Department of Education, National Center for Education Statistics, Integrated Postsecondary Education Data System, "Fall Enrollment Survey" (IPEDS-EF:95-99), and Spring 2001 through Spring 2010; Enrollment in Degree-Granting Institutions Model, 1980-2009. (This figure was prepared March 2011.)

\section{Enrollment by race/ ethnicity}

Between 2009 and 2020, enrollment is projected to

$\Delta$ increase 1 percent for students who are White;

$\Delta$ increase 25 percent for students who are Black;

A increase 46 percent for students who are Hispanic;

$\Delta$ increase 25 percent for students who are Asian/Pacific Islander; and

$\nabla$ decrease 1 percent for students who are American Indian/Alaska Native.

For more information:

Table 29

\section{Enrollment in public and private institutions}

Between 2009 and 2020, enrollment is projected to increase

$\Delta 13$ percent in public institutions; and

A 13 percent in private institutions.

For more information:

Table 20 
Total first-time freshmen enrollment in degree-granting institutions increased 48 percent from 1995 to 2009.

Between 2009 and 2020, first-time freshmen enrollment is projected to increase

$\Delta 11$ percent overall;

4 6 percent for men; and

$\Delta 15$ percent for women.

For more information:

Table 30
Figure 23. Actual and projected numbers for total first-time freshmen enrollment in all postsecondary degree-granting institutions, by sex: Fall 1995 through fall 2020

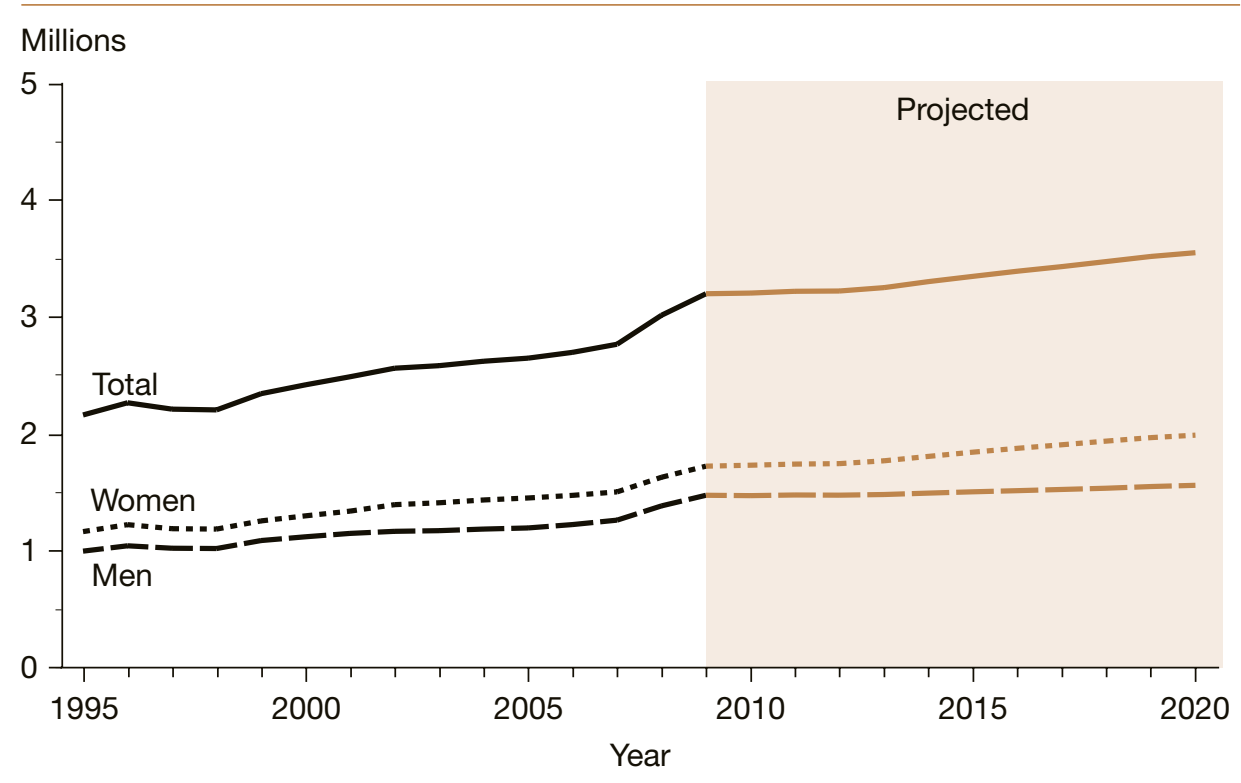

NOTE: Some data have been revised from previously published figures. Mean absolute percentage errors of selected education statistics can be found in table A-2, appendix A. SOURCE: U.S. Department of Education, National Center for Education Statistics, Integrated Postsecondary Education Data System, "Fall Enrollment Survey" (IPEDS-EF:95-99), and Spring 2001 through Spring 2010; Enrollment in Degree-Granting Institutions Model, 1980-2009; and First-Time Freshmen Model, 1975-2009. (This figure was prepared March 2011.) 


\section{Section 6 \\ Postsecondary Degrees Conferred}

\section{INTRODUCTION}

Continuing growth in enrollment in postsecondary degree-granting institutions has been reflected by an increase in the number of degrees conferred. Increases in the number of degrees conferred are expected to continue between academic year 2008-09, the last year of actual data, and academic year 2020-21.

\section{Factors affecting the projections}

The projections of the number of degrees conferred are related to projections of the college-age populations developed by the Census Bureau and college enrollments from this report. For more details, see appendixes A.0 and A.6.

\section{Factors that were not considered}

Some factors that may affect future numbers of degrees, such as choice of degree and labor force requirements, were not included in the projection models.

\section{About first-professional degrees}

A first-professional degree is one that signifies both completion of the academic requirements for beginning practice in a given profession and a level of professional skill beyond that required for a bachelor's degree. A first-professional degree is based on a program requiring at least 2 academic years of work beyond the bachelor's degree. Degree fields include dentistry, medicine, optometry, osteopathic medicine, pharmacy, podiatric medicine, veterinary medicine, chiropractic, law, and theological professions.

\section{Accuracy of Projections}

No MAPEs were calculated for degrees conferred as the current model used for producing their projections has been used for only two other editions of Projections of Education Statistics. For more information on the MAPEs of different National Center for Education Statistics (NCES) projection series, see table A-2 in appendix A. 


\section{DEGREES, BY LEVEL OF DEGREE AND SEX OF RECIPIENT}

\section{Associate's degrees}

Between 2008-09 and 2020-21, the number of associate's degrees is projected to increase

$\Delta 26$ percent overall;

$\Delta 23$ percent for men; and

- 28 percent for women.

For more information:

Table 32

\section{Bachelor's degrees}

Between 2008-09 and 2020-21, the number of bachelor's degrees is projected to increase

- 21 percent overall;

$\Delta \quad 18$ percent for men; and

$\Delta 24$ percent for women.

For more information:

Table 33
Figure 24. Actual and projected numbers for associate's degrees conferred by postsecondary degree-granting institutions, by sex of recipient: 1995-96 through 2020-21

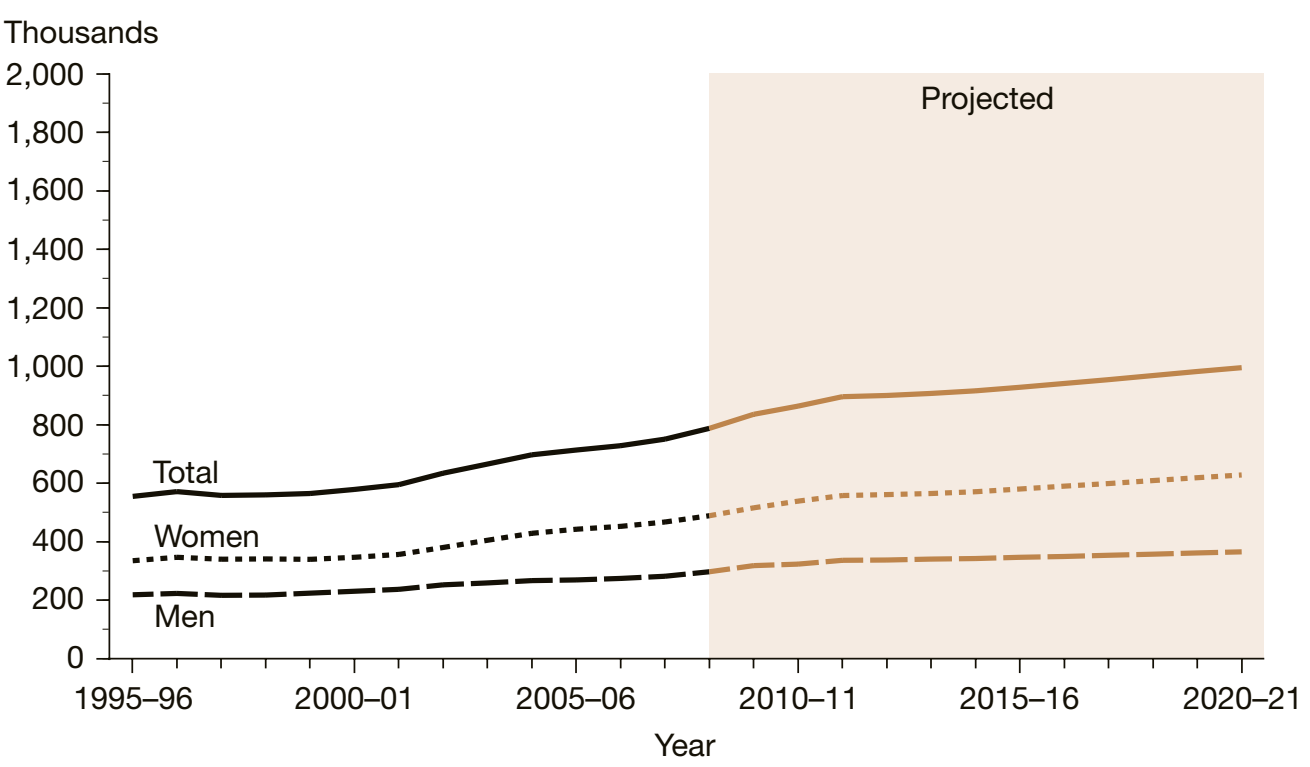

NOTE: Some data have been revised from previously published figures. Mean absolute percentage errors of selected education statistics can be found in table A-2, appendix A. SOURCE: U.S. Department of Education, National Center for Education Statistics, Integrated Postsecondary Education Data System, "Completions Survey" (IPEDS-C:95-99), and Fall 2000 through Fall 2009; and Degrees Conferred Model, 1975-76 through 2008-09. (This figure was prepared April 2011.)

Figure 25. Actual and projected numbers for bachelor's degrees conferred by postsecondary degree-granting institutions, by sex of recipient: 1995-96 through 2020-21

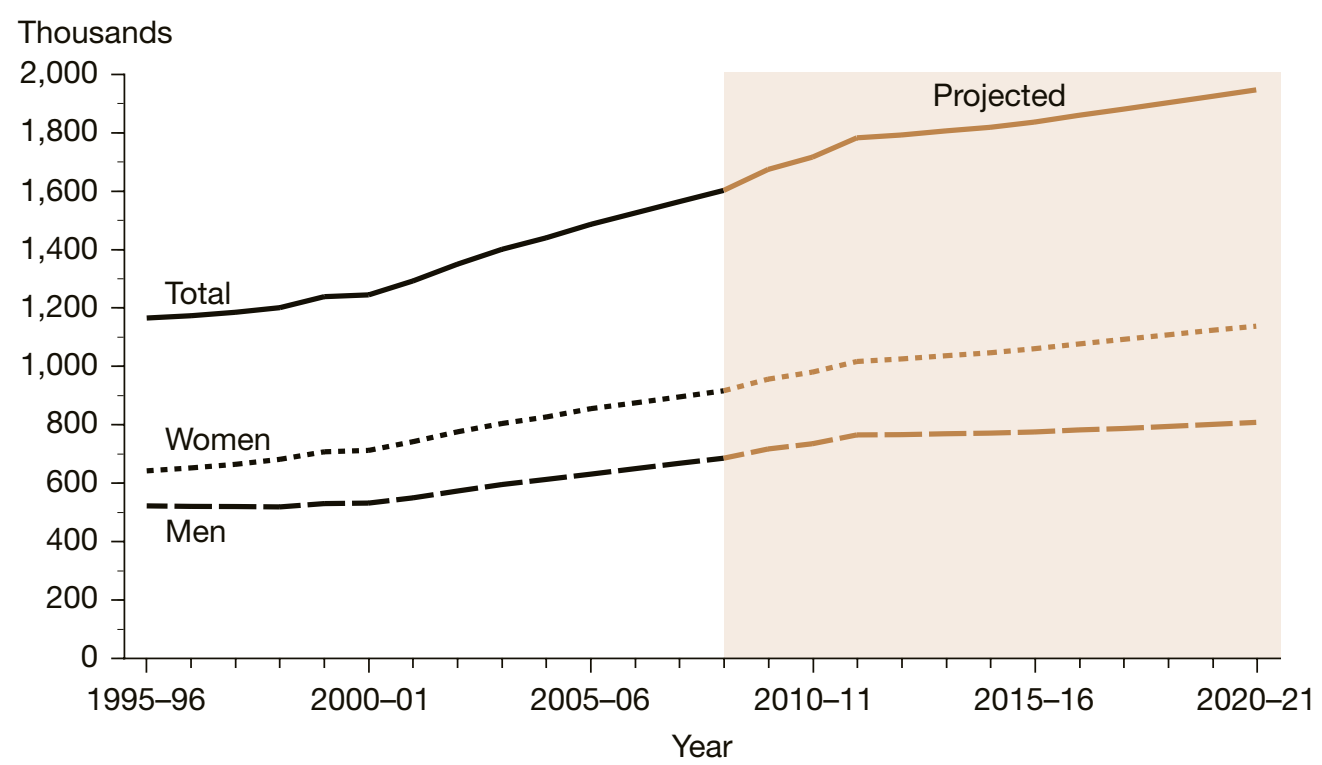

NOTE: Some data have been revised from previously published figures. Mean absolute percentage errors of selected education statistics can be found in table A-2, appendix A. SOURCE: U.S. Department of Education, National Center for Education Statistics, Integrated Postsecondary Education Data System, "Completions Survey" (IPEDS-C:95-99), and Fall 2000 through Fall 2009; and Degrees Conferred Model, 1975-76 through 2008-09. (This figure was prepared April 2011.) 
Figure 26. Actual and projected numbers for master's degrees conferred by postsecondary degree-granting institutions, by sex of recipient: 1995-96 through 2020-21

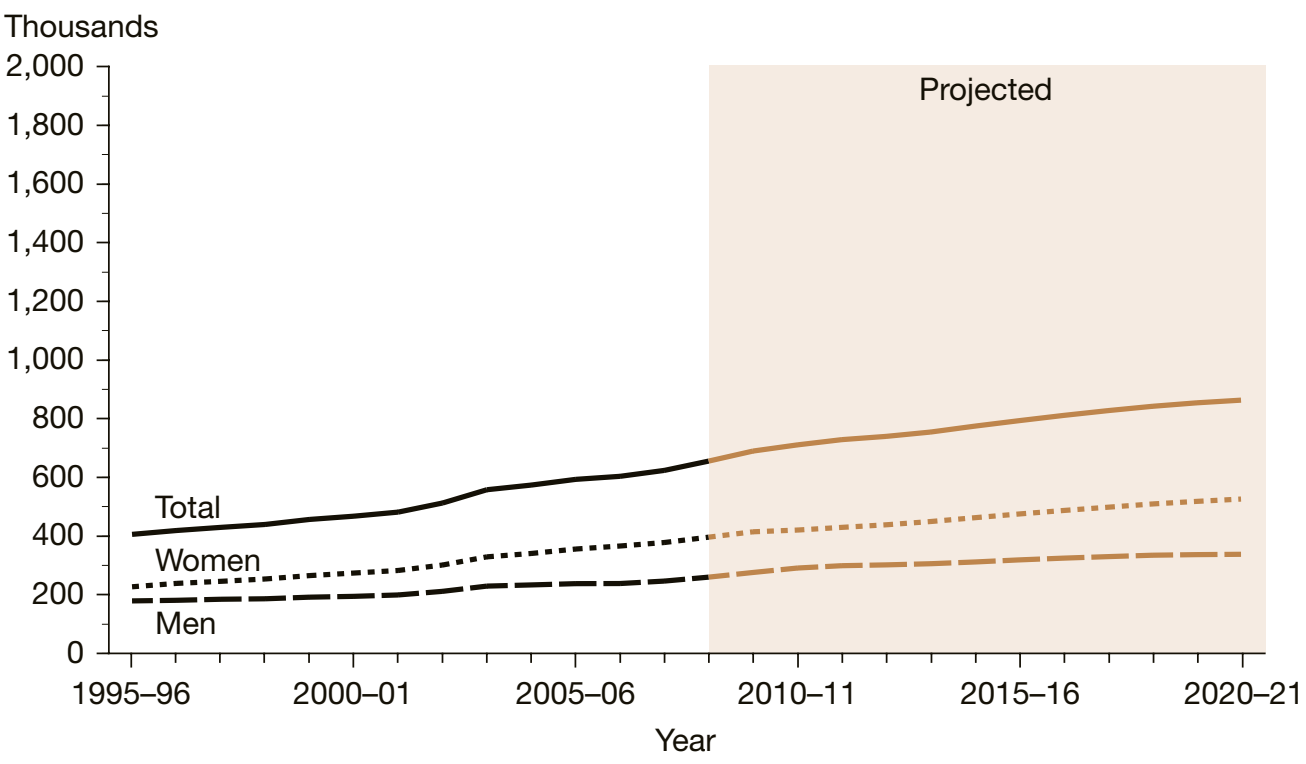

NOTE: Some data have been revised from previously published figures. Mean absolute percentage errors of selected education statistics can be found in table A-2, appendix A. SOURCE: U.S. Department of Education, National Center for Education Statistics, Integrated Postsecondary Education Data System, "Completions Survey" (IPEDS-C:95-99), and Fall 2000 through Fall 2009; and Degrees Conferred Model, 1975-76 through 2008-09. (This figure was prepared April 2011.)

Figure 27. Actual and projected numbers for doctor's degrees conferred by postsecondary degree-granting institutions, by sex of recipient: 1995-96 through 2020-21

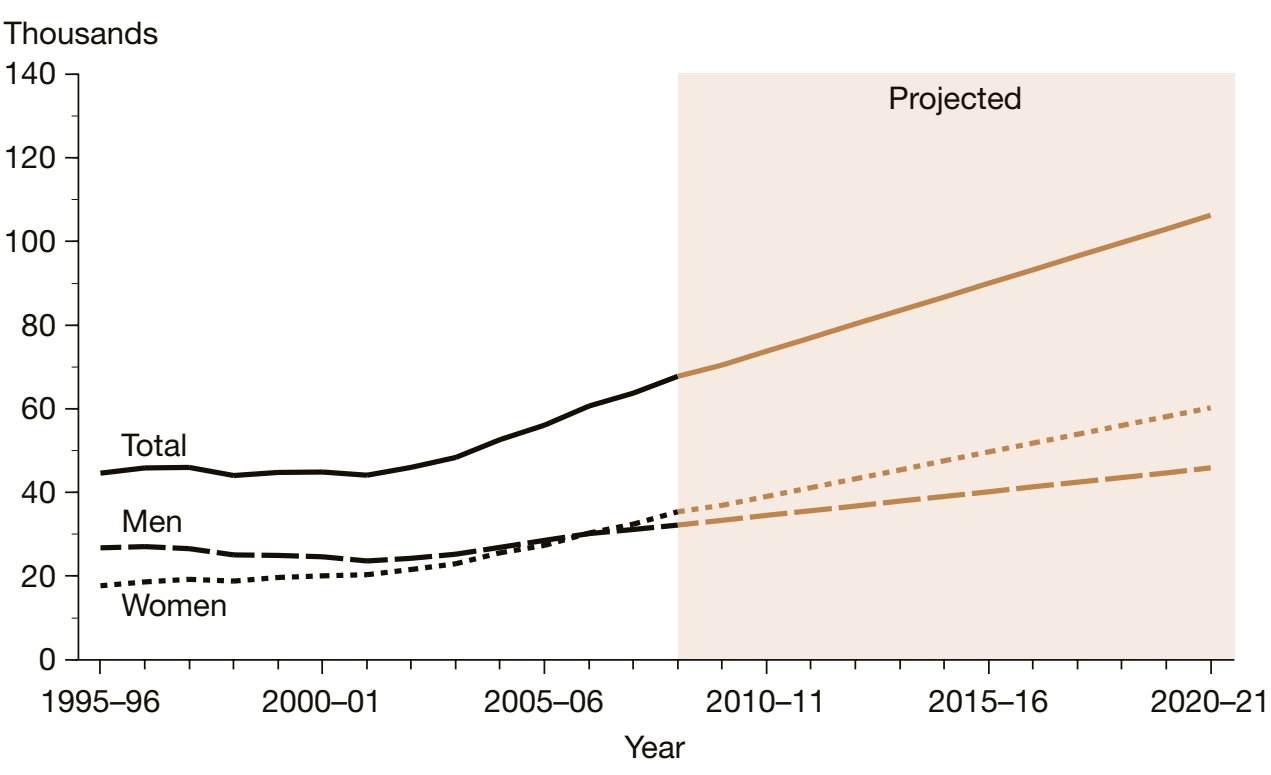

NOTE: Doctor's degrees include Ph.D., Ed.D., and comparable degrees at the doctoral level. Excluded are first-professional degrees, such as M.D., D.D.S., and law degrees. Some data have been revised from previously published figures. Mean absolute percentage errors of selected education statistics can be found in table A-2, appendix A.

SOURCE: U.S. Department of Education, National Center for Education Statistics, Integrated Postsecondary Education Data System, "Completions Survey" (IPEDS-C:95-99), and Fall 2000 through Fall 2009; and Degrees Conferred Model, 1975-76 through 2008-09. (This figure was prepared April 2011.)

\section{Master's degrees}

Between 2008-09 and 2020-21, the number of master's degrees is projected to increase

$\Delta 32$ percent overall;

- 30 percent for men; and

- 33 percent for women.

For more information:

Table 34

\section{Doctor's degrees}

Between 2008-09 and 2020-21, the number of doctor's degrees is projected to increase
- 57 percent overall;
$4 \quad 42$ percent for men; and
70 percent for women.

For more information:

Table 35 


\section{First-professional degrees}

Between 2008-09 and 2020-21, the number of first-professional degrees is projected to increase

$\Delta 30$ percent overall;

$\Delta 26$ percent for men; and

- 33 percent for women.

\section{For more information:}

Table 36
Figure 28. Actual and projected numbers for first-professional degrees conferred by postsecondary degree-granting institutions, by sex of recipient: 1995-96 through 2020-21

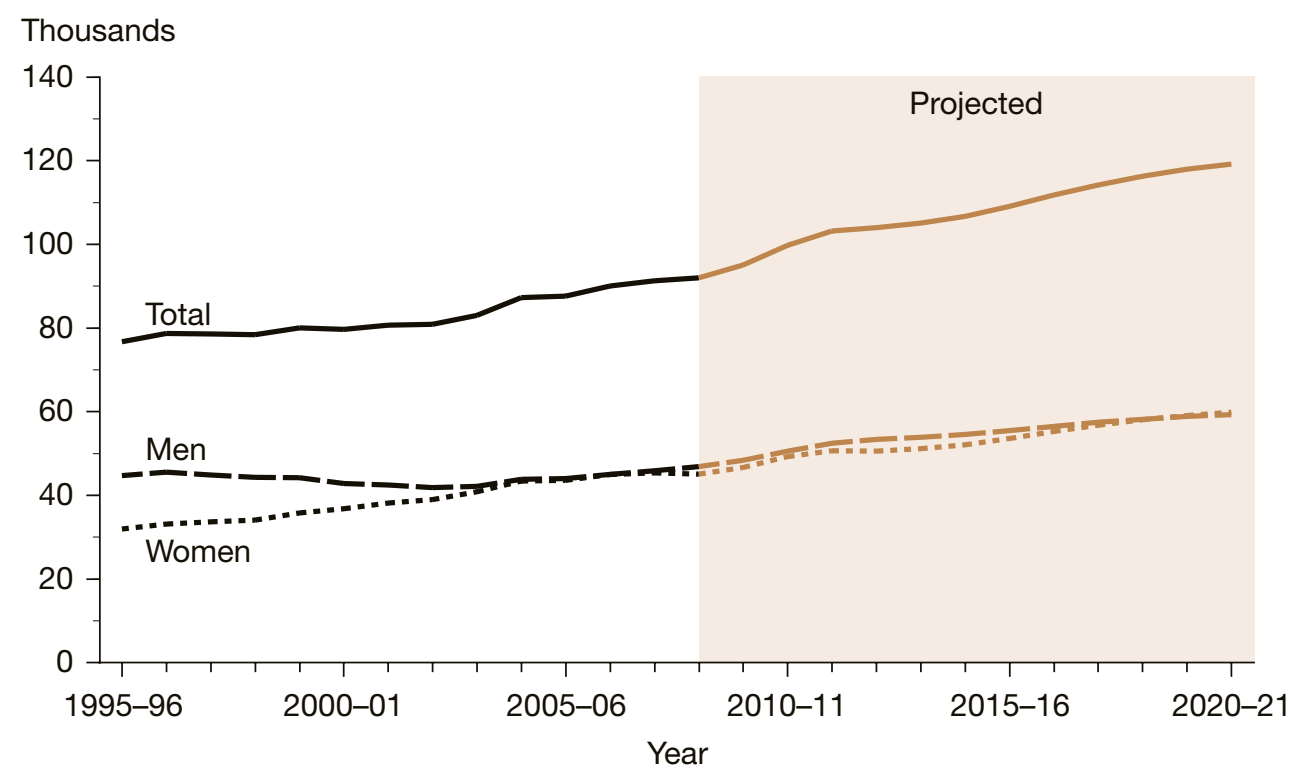

NOTE: Some data have been revised from previously published figures. Mean absolute percentage errors of selected education statistics can be found in table A-2, appendix A. SOURCE: U.S. Department of Education, National Center for Education Statistics, Integrated Postsecondary Education Data System, "Completions Survey" (IPEDS-C:95-99), and Fall 2000 through Fall 2009; and Degrees Conferred Model, 1975-76 through 2008-09. (This figure was prepared April 2011.) 


\section{Reference Tables}


This page intentionally left blank. 
Table 1. Actual and projected numbers for enrollment in grades PK-12, PK-8, and 9-12 in elementary and secondary schools, by control of school: Fall 1995 through fall 2020

[In thousands]

\begin{tabular}{|c|c|c|c|c|c|c|c|c|c|}
\hline \multirow[b]{2}{*}{ Year } & \multicolumn{3}{|c|}{ Total } & \multicolumn{3}{|c|}{ Public } & \multicolumn{3}{|c|}{ Private } \\
\hline & $\mathrm{PK}-12^{1}$ & $\mathrm{PK}-8^{1}$ & $9-12$ & PK-12 & PK-8 & $9-12$ & PK-12 & $\mathrm{PK}-8$ & $9-12$ \\
\hline \multicolumn{10}{|c|}{ Actual } \\
\hline 1995 & 50,759 & 37,059 & 13,699 & 44,840 & 32,338 & 12,502 & 5,918 & 4,721 & 1,197 \\
\hline $1996^{2}$ & 51,544 & 37,481 & 14,062 & 45,611 & 32,762 & 12,849 & 5,933 & 4,719 & 1,213 \\
\hline 1997 & 52,071 & 37,797 & 14,275 & 46,127 & 33,071 & 13,056 & 5,944 & 4,726 & 1,219 \\
\hline $1998^{2}$ & 52,526 & 38,091 & 14,435 & 46,539 & 33,344 & 13,195 & 5,988 & 4,747 & 1,240 \\
\hline 1999 & 52,875 & 38,251 & 14,625 & 46,857 & 33,486 & 13,371 & 6,018 & 4,764 & 1,254 \\
\hline $2000^{2}$ & 53,373 & 38,564 & 14,809 & 47,204 & 33,686 & 13,517 & 6,169 & 4,877 & 1,292 \\
\hline 2001 & 53,992 & 38,929 & 15,063 & 47,672 & 33,936 & 13,736 & 6,320 & 4,993 & 1,327 \\
\hline $2002^{2}$ & 54,403 & 39,000 & 15,404 & 48,183 & 34,114 & 14,069 & 6,220 & 4,886 & 1,335 \\
\hline 2003 & 54,639 & 38,962 & 15,678 & 48,540 & 34,201 & 14,339 & 6,099 & 4,761 & 1,338 \\
\hline $2004^{2}$ & 54,882 & 38,908 & 15,974 & 48,795 & 34,178 & 14,618 & 6,087 & 4,731 & 1,356 \\
\hline 2005 & 55,187 & 38,903 & 16,283 & 49,113 & 34,204 & 14,909 & 6,073 & 4,699 & 1,374 \\
\hline $2006^{2}$ & 55,307 & 38,838 & 16,469 & 49,316 & 34,235 & 15,081 & 5,991 & 4,604 & 1,388 \\
\hline 2007 & 55,203 & 38,722 & 16,481 & 49,293 & 34,205 & 15,087 & 5,910 & 4,517 & 1,394 \\
\hline $2008^{2}$ & 54,972 & 38,620 & 16,352 & 49,265 & 34,285 & 14,980 & 5,707 & 4,335 & 1,373 \\
\hline \multicolumn{10}{|c|}{ Projected } \\
\hline 2009 & 54,770 & 38,592 & 16,179 & 49,282 & 34,440 & 14,842 & 5,488 & 4,151 & 1,337 \\
\hline 2010 & 54,704 & 38,729 & 15,975 & 49,306 & 34,637 & 14,668 & 5,398 & 4,092 & 1,306 \\
\hline 2011 & 54,746 & 38,949 & 15,797 & 49,422 & 34,892 & 14,530 & 5,324 & 4,057 & 1,266 \\
\hline 2012 & 54,905 & 39,163 & 15,742 & 49,642 & 35,129 & 14,512 & 5,263 & 4,034 & 1,229 \\
\hline 2013 & 55,133 & 39,394 & 15,739 & 49,914 & 35,368 & 14,545 & 5,219 & 4,025 & 1,194 \\
\hline 2014 & 55,455 & 39,606 & 15,849 & 50,268 & 35,579 & 14,689 & 5,187 & 4,027 & 1,160 \\
\hline 2015 & 55,836 & 39,872 & 15,964 & 50,659 & 35,829 & 14,830 & 5,176 & 4,042 & 1,134 \\
\hline 2016 & 56,214 & 40,234 & 15,980 & 51,038 & 36,161 & 14,877 & 5,176 & 4,073 & 1,103 \\
\hline 2017 & 56,617 & 40,602 & 16,015 & 51,430 & 36,491 & 14,939 & 5,187 & 4,110 & 1,077 \\
\hline 2018 & 57,009 & 40,949 & 16,060 & 51,803 & 36,803 & 15,000 & 5,206 & 4,146 & 1,060 \\
\hline 2019 & 57,438 & 41,303 & 16,135 & 52,204 & 37,121 & 15,083 & 5,234 & 4,181 & 1,052 \\
\hline 2020 & 57,939 & 41,661 & 16,278 & 52,666 & 37,444 & 15,222 & 5,273 & 4,216 & 1,056 \\
\hline
\end{tabular}

${ }^{1}$ Includes private nursery and prekindergarten enrollment in schools that offer kindergarten or higher grades.

${ }^{2}$ Since the biennial Private School Universe Survey (PSS) is collected in the fall of odd numbered years, private school numbers for alternate years are estimated based on data from the PSS.

NOTE: PK=prekindergarten. Some data have been revised from previously published figures. Detail may not sum to totals because of rounding. Mean absolute percentage errors of selected education statistics can be found in table A-2, appendix $A$.

SOURCE: U.S. Department of Education, National Center for Education Statistics, Common Core of Data (CCD), "State Nonfiscal Survey of Public Elementary/Secondary Education," 1995-96 through 2008-09; Private School Universe Survey (PSS), selected years 1995-96 through 2009-10; and National Elementary and Secondary Enrollment Model, 1972-2008. (This table was prepared January 2011.) 
Table 2. Actual and projected numbers for enrollment in public elementary and secondary schools, by grade: Fall 1995 through fall 2020

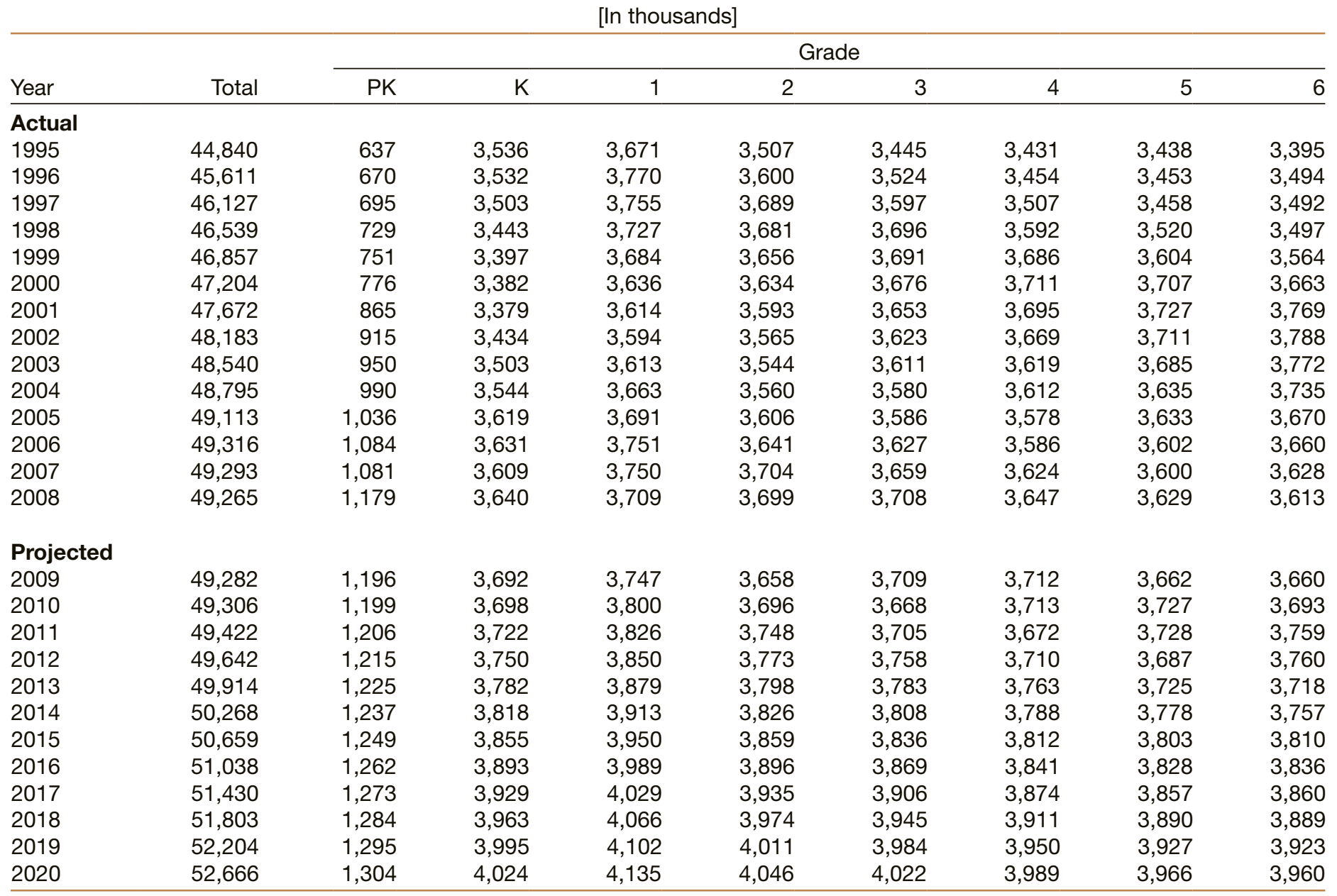

See notes at end of table. 
Table 2. Actual and projected numbers for enrollment in public elementary and secondary schools, by grade: Fall 1995 through fall 2020-Continued

[In thousands]

\begin{tabular}{|c|c|c|c|c|c|c|c|c|}
\hline \multirow[b]{2}{*}{ Year } & \multicolumn{6}{|c|}{ Grade } & \multirow{2}{*}{$\begin{array}{r}\text { Elementary } \\
\text { ungraded }\end{array}$} & \multirow{2}{*}{$\begin{array}{r}\text { Secondary } \\
\text { ungraded }\end{array}$} \\
\hline & 7 & 8 & 9 & 10 & 11 & 12 & & \\
\hline \multicolumn{9}{|c|}{ Actual } \\
\hline 1995 & 3,422 & 3,356 & 3,704 & 3,237 & 2,826 & 2,487 & 500 & 247 \\
\hline 1996 & 3,464 & 3,403 & 3,801 & 3,323 & 2,930 & 2,586 & 399 & 208 \\
\hline 1997 & 3,520 & 3,415 & 3,819 & 3,376 & 2,972 & 2,673 & 440 & 216 \\
\hline 1998 & 3,530 & 3,480 & 3,856 & 3,382 & 3,021 & 2,722 & 449 & 214 \\
\hline 1999 & 3,541 & 3,497 & 3,935 & 3,415 & 3,034 & 2,782 & 415 & 205 \\
\hline 2000 & 3,629 & 3,538 & 3,963 & 3,491 & 3,083 & 2,803 & 334 & 177 \\
\hline 2001 & 3,720 & 3,616 & 4,012 & 3,528 & 3,174 & 2,863 & 304 & 159 \\
\hline 2002 & 3,821 & 3,709 & 4,105 & 3,584 & 3,229 & 2,990 & 285 & 161 \\
\hline 2003 & 3,841 & 3,809 & 4,190 & 3,675 & 3,277 & 3,046 & 255 & 150 \\
\hline 2004 & 3,818 & 3,825 & 4,281 & 3,750 & 3,369 & 3,094 & 215 & 122 \\
\hline 2005 & 3,777 & 3,802 & 4,287 & 3,866 & 3,454 & 3,180 & 205 & 121 \\
\hline 2006 & 3,716 & 3,766 & 4,260 & 3,882 & 3,551 & 3,277 & 170 & 110 \\
\hline 2007 & 3,701 & 3,709 & 4,200 & 3,863 & 3,558 & 3,375 & 139 & 92 \\
\hline 2008 & 3,653 & 3,692 & 4,122 & 3,822 & 3,548 & 3,400 & 118 & 88 \\
\hline \multicolumn{9}{|c|}{ Projected } \\
\hline 2009 & 3,643 & 3,644 & 4,103 & 3,752 & 3,510 & 3,390 & 118 & 86 \\
\hline 2010 & 3,691 & 3,635 & 4,049 & 3,734 & 3,446 & 3,355 & 119 & 85 \\
\hline 2011 & 3,724 & 3,681 & 4,039 & 3,685 & 3,429 & 3,293 & 120 & 85 \\
\hline 2012 & 3,791 & 3,715 & 4,091 & 3,676 & 3,384 & 3,277 & 121 & 84 \\
\hline 2013 & 3,791 & 3,781 & 4,128 & 3,723 & 3,376 & 3,234 & 123 & 84 \\
\hline 2014 & 3,750 & 3,782 & 4,202 & 3,757 & 3,420 & 3,226 & 124 & 84 \\
\hline 2015 & 3,788 & 3,740 & 4,203 & 3,824 & 3,450 & 3,268 & 125 & 85 \\
\hline 2016 & 3,842 & 3,779 & 4,157 & 3,825 & 3,512 & 3,297 & 126 & 86 \\
\hline 2017 & 3,868 & 3,832 & 4,199 & 3,783 & 3,513 & 3,356 & 127 & 87 \\
\hline 2018 & 3,893 & 3,858 & 4,259 & 3,822 & 3,474 & 3,357 & 128 & 88 \\
\hline 2019 & 3,922 & 3,883 & 4,288 & 3,876 & 3,510 & 3,320 & 130 & 89 \\
\hline 2020 & 3,956 & 3,912 & 4,315 & 3,902 & 3,560 & 3,354 & 131 & 90 \\
\hline
\end{tabular}

NOTE: PK=prekindergarten. K=kindergarten. Elementary ungraded includes students in grades prekindergarten through 8 who are in classes or programs to which students are assigned without standard grade designations. Secondary ungraded includes students in grades 9 through 12 who are in classes or programs to which students are assigned without standard grade designations. Some data have been revised from previously published figures. Detail may not sum to totals because of rounding. Mean absolute percentage errors of selected education statistics can be found in table A-2, appendix A.

SOURCE: U.S. Department of Education, National Center for Education Statistics, Common Core of Data (CCD), "State Nonfiscal Survey of Public Elementary/Secondary Education," 1995-96 through 2008-09; and National Elementary and Secondary Enrollment Model, 1972-2008. (This table was prepared January 2011.) 
Table 3. Actual and projected numbers for enrollment in public elementary and secondary schools, by race/ethnicity: Fall 1995 through fall 2020

\begin{tabular}{|c|c|c|c|c|c|c|}
\hline \multirow[b]{2}{*}{ Year } & \multirow[b]{2}{*}{ Total } & \multicolumn{5}{|c|}{ Race/ethnicity } \\
\hline & & White & Black & Hispanic & $\begin{array}{r}\text { Asian/Pacific } \\
\text { Islander }\end{array}$ & $\begin{array}{r}\text { American Indian/ } \\
\text { Alaska Native }\end{array}$ \\
\hline \multicolumn{7}{|c|}{ Actual } \\
\hline 1995 & 44,840 & 29,030 & 7,552 & 6,085 & 1,668 & 505 \\
\hline 1996 & 45,611 & 29,217 & 7,708 & 6,429 & 1,731 & 527 \\
\hline 1997 & 46,127 & 29,241 & 7,851 & 6,705 & 1,796 & 535 \\
\hline 1998 & 46,539 & 29,217 & 7,935 & 7,007 & 1,846 & 534 \\
\hline 1999 & 46,857 & 29,032 & 8,054 & 7,337 & 1,892 & 542 \\
\hline 2000 & 47,204 & 28,873 & 8,099 & 7,733 & 1,949 & 550 \\
\hline 2001 & 47,672 & 28,731 & 8,176 & 8,175 & 2,026 & 563 \\
\hline 2002 & 48,183 & 28,614 & 8,297 & 8,601 & 2,088 & 583 \\
\hline 2003 & 48,540 & 28,438 & 8,347 & 9,018 & 2,144 & 593 \\
\hline 2004 & 48,795 & 28,186 & 8,400 & 9,415 & 2,204 & 591 \\
\hline 2005 & 49,113 & 28,001 & 8,443 & 9,794 & 2,278 & 598 \\
\hline 2006 & 49,316 & 27,797 & 8,421 & 10,171 & 2,331 & 595 \\
\hline 2007 & 49,293 & 27,454 & 8,392 & 10,457 & 2,396 & 594 \\
\hline $2008^{1}$ & 49,265 & 27,191 & 8,399 & 10,621 & 2,461 & 592 \\
\hline \multicolumn{7}{|c|}{ Projected } \\
\hline 2009 & 49,282 & 26,998 & 8,346 & 10,800 & 2,537 & 594 \\
\hline 2010 & 49,306 & 26,827 & 8,291 & 10,963 & 2,614 & 597 \\
\hline 2011 & 49,422 & 26,716 & 8,251 & 11,139 & 2,694 & 602 \\
\hline 2012 & 49,642 & 26,658 & 8,239 & 11,334 & 2,775 & 610 \\
\hline 2013 & 49,914 & 26,628 & 8,244 & 11,534 & 2,856 & 619 \\
\hline 2014 & 50,268 & 26,634 & 8,278 & 11,753 & 2,935 & 629 \\
\hline 2015 & 50,659 & 26,664 & 8,315 & 11,982 & 3,012 & 640 \\
\hline 2016 & 51,038 & 26,687 & 8,344 & 12,217 & 3,090 & 650 \\
\hline 2017 & 51,430 & 26,710 & 8,372 & 12,470 & 3,165 & 660 \\
\hline 2018 & 51,803 & 26,728 & 8,399 & 12,729 & 3,225 & 670 \\
\hline 2019 & 52,204 & 26,755 & 8,435 & 12,999 & 3,284 & 680 \\
\hline 2020 & 52,666 & 26,814 & 8,481 & 13,289 & 3,338 & 691 \\
\hline
\end{tabular}

${ }^{1}$ In 2008, five states reported enrollment counts for students of two or more races. These enrollment counts were proportioned across the other racial/ethnic categories. When more complete sets of data for students of two or more races are compiled, separate projections for that category will be presented.

NOTE: Some data have been revised from previously published figures. Race categories exclude persons of Hispanic ethnicity. The historical racial/ ethnic time-series were constructed using racial/ethnic enrollment data at the state level for individual grades. In some instances, enrollment data by race/ethnicity had to be imputed. Further, in some instances, the racial/ethnic enrollment data for individual grades had to be adjusted in order for them to sum to the state total for that grade. For additional information, see the Elementary and Secondary Enrollment section A.1 in appendix A. Detail may not sum to totals because of rounding. Mean absolute percentage errors of selected education statistics can be found in table A-2, appendix A. SOURCE: U.S. Department of Education, National Center for Education Statistics, Common Core of Data (CCD), "State Nonfiscal Survey of Public Elementary/Secondary Education," 1995-96 through 2008-09; and National Public Elementary and Secondary Enrollment by Race/Ethnicity Model, 1994-2008. (This table was prepared January 2011.) 
Table 4. Actual and projected numbers for enrollment in grades PK-8 in public elementary and secondary schools, by race/ ethnicity: Fall 1995 through fall 2020

\begin{tabular}{|c|c|c|c|c|c|c|}
\hline \multirow[b]{2}{*}{ Year } & \multirow[b]{2}{*}{ Total } & \multicolumn{5}{|c|}{ Race/ethnicity } \\
\hline & & White & Black & Hispanic & $\begin{array}{r}\text { Asian/Pacific } \\
\text { Islander }\end{array}$ & $\begin{array}{r}\text { American Indian/ } \\
\text { Alaska Native } \\
\end{array}$ \\
\hline \multicolumn{7}{|c|}{ Actual } \\
\hline 1995 & 32,338 & 20,684 & 5,549 & 4,565 & 1,170 & 371 \\
\hline 1996 & 32,762 & 20,687 & 5,664 & 4,821 & 1,204 & 386 \\
\hline 1997 & 33,071 & 20,625 & 5,782 & 5,030 & 1,244 & 390 \\
\hline 1998 & 33,344 & 20,548 & 5,861 & 5,274 & 1,275 & 386 \\
\hline 1999 & 33,486 & 20,313 & 5,948 & 5,529 & 1,305 & 391 \\
\hline 2000 & 33,686 & 20,123 & 5,980 & 5,838 & 1,348 & 397 \\
\hline 2001 & 33,936 & 19,954 & 6,002 & 6,167 & 1,408 & 405 \\
\hline 2002 & 34,114 & 19,760 & 6,040 & 6,453 & 1,446 & 415 \\
\hline 2003 & 34,201 & 19,554 & 6,013 & 6,736 & 1,482 & 415 \\
\hline 2004 & 34,178 & 19,266 & 5,992 & 6,988 & 1,519 & 413 \\
\hline 2005 & 34,204 & 19,047 & 5,953 & 7,223 & 1,569 & 412 \\
\hline 2006 & 34,235 & 18,859 & 5,880 & 7,470 & 1,611 & 414 \\
\hline 2007 & 34,205 & 18,678 & 5,821 & 7,636 & 1,660 & 412 \\
\hline $2008^{1}$ & 34,285 & 18,600 & 5,824 & 7,737 & 1,712 & 412 \\
\hline \multicolumn{7}{|c|}{ Projected } \\
\hline 2009 & 34,440 & 18,582 & 5,792 & 7,869 & 1,773 & 418 \\
\hline 2010 & 34,637 & 18,572 & 5,794 & 8,005 & 1,830 & 424 \\
\hline 2011 & 34,892 & 18,592 & 5,804 & 8,156 & 1,889 & 432 \\
\hline 2012 & 35,129 & 18,600 & 5,809 & 8,310 & 1,949 & 438 \\
\hline 2013 & 35,368 & 18,599 & 5,814 & 8,475 & 2,008 & 445 \\
\hline 2014 & 35,579 & 18,590 & 5,818 & 8,641 & 2,052 & 452 \\
\hline 2015 & 35,829 & 18,597 & 5,831 & 8,821 & 2,096 & 459 \\
\hline 2016 & 36,161 & 18,648 & 5,858 & 9,026 & 2,136 & 468 \\
\hline 2017 & 36,491 & 18,689 & 5,906 & 9,223 & 2,171 & 477 \\
\hline 2018 & 36,803 & 18,710 & 5,967 & 9,416 & 2,201 & 484 \\
\hline 2019 & 37,121 & 18,737 & 6,024 & 9,615 & 2,228 & 492 \\
\hline 2020 & 37,444 & 18,769 & 6,077 & 9,820 & 2,255 & 498 \\
\hline
\end{tabular}

${ }^{1}$ In 2008, five states reported enrollment counts for students of two or more races. These enrollment counts were proportioned across the other racial/ethnic categories. When more complete sets of data for students of two or more races are compiled, separate projections for that category will be presented.

NOTE: Some data have been revised from previously published figures. The historical racial/ethnic time-series were constructed using racial/ethnic enrollment data at the state level for individual grades. In some instances, enrollment data by race/ethnicity had to be imputed. Further, in some instances, the racial/ethnic enrollment data for individual grades had to be adjusted in order for them to sum to the state total for that grade. Race categories exclude persons of Hispanic ethnicity. For additional information, see the Elementary and Secondary Enrollment section A.1 in appendix A. Detail may not sum to totals because of rounding. Mean absolute percentage errors of selected education statistics can be found in table A-2, appendix A.

SOURCE: U.S. Department of Education, National Center for Education Statistics, Common Core of Data (CCD), "State Nonfiscal Survey of Public Elementary/Secondary Education," 1995-96 through 2008-09; and National Public Elementary and Secondary Enrollment by Race/Ethnicity Model, 1994-2008. (This table was prepared January 2011.) 
Table 5. Actual and projected numbers for enrollment in grades 9-12 in public schools, by race/ethnicity: Fall 1995 through fall 2020

\begin{tabular}{|c|c|c|c|c|c|c|}
\hline \multirow[b]{2}{*}{ Year } & \multirow[b]{2}{*}{ Total } & \multicolumn{5}{|c|}{ Race/ethnicity } \\
\hline & & White & Black & Hispanic & $\begin{array}{r}\text { Asian/Pacific } \\
\text { Islander }\end{array}$ & $\begin{array}{r}\text { American Indian/ } \\
\text { Alaska Native }\end{array}$ \\
\hline \multicolumn{7}{|c|}{ Actual } \\
\hline 1995 & 12,502 & 8,347 & 2,002 & 1,520 & 498 & 135 \\
\hline 1996 & 12,849 & 8,530 & 2,043 & 1,608 & 526 & 141 \\
\hline 1997 & 13,056 & 8,616 & 2,068 & 1,675 & 552 & 145 \\
\hline 1998 & 13,195 & 8,670 & 2,073 & 1,732 & 572 & 148 \\
\hline 1999 & 13,371 & 8,719 & 2,106 & 1,808 & 587 & 151 \\
\hline 2000 & 13,517 & 8,750 & 2,119 & 1,894 & 601 & 153 \\
\hline 2001 & 13,736 & 8,777 & 2,173 & 2,008 & 619 & 158 \\
\hline 2002 & 14,069 & 8,854 & 2,257 & 2,148 & 642 & 168 \\
\hline 2003 & 14,339 & 8,884 & 2,334 & 2,282 & 663 & 177 \\
\hline 2004 & 14,618 & 8,920 & 2,408 & 2,427 & 686 & 178 \\
\hline 2005 & 14,909 & 8,954 & 2,490 & 2,570 & 709 & 186 \\
\hline 2006 & 15,081 & 8,938 & 2,540 & 2,701 & 720 & 181 \\
\hline 2007 & 15,087 & 8,776 & 2,571 & 2,821 & 736 & 183 \\
\hline $2008^{1}$ & 14,980 & 8,590 & 2,575 & 2,885 & 749 & 180 \\
\hline \multicolumn{7}{|c|}{ Projected } \\
\hline 2009 & 14,842 & 8,416 & 2,553 & 2,931 & 764 & 176 \\
\hline 2010 & 14,668 & 8,254 & 2,497 & 2,958 & 784 & 172 \\
\hline 2011 & 14,530 & 8,125 & 2,446 & 2,984 & 806 & 170 \\
\hline 2012 & 14,512 & 8,058 & 2,430 & 3,024 & 826 & 171 \\
\hline 2013 & 14,545 & 8,029 & 2,430 & 3,059 & 848 & 174 \\
\hline 2014 & 14,689 & 8,044 & 2,460 & 3,111 & 883 & 177 \\
\hline 2015 & 14,830 & 8,066 & 2,484 & 3,161 & 916 & 180 \\
\hline 2016 & 14,877 & 8,040 & 2,486 & 3,191 & 954 & 181 \\
\hline 2017 & 14,939 & 8,021 & 2,466 & 3,247 & 993 & 183 \\
\hline 2018 & 15,000 & 8,018 & 2,432 & 3,314 & 1,024 & 186 \\
\hline 2019 & 15,083 & 8,018 & 2,410 & 3,384 & 1,055 & 189 \\
\hline 2020 & 15,222 & 8,046 & 2,403 & 3,470 & 1,083 & 193 \\
\hline
\end{tabular}

${ }^{1}$ In 2008, five states reported enrollment counts for students of two or more races. These enrollment counts were proportioned across the other racial/ethnic categories. When more complete sets of data for students of two or more races are compiled, separate projections for that category will be presented.

NOTE: Some data have been revised from previously published figures. The historical racial/ethnic time-series were constructed using racial/ ethnic enrollment data at the state level for individual grades. In some instances, enrollment data by race/ethnicity had to be imputed. Further, in some instances, the racial/ethnic enrollment data for individual grades had to be adjusted in order for them to sum to the state total for that grade. Race categories exclude persons of Hispanic ethnicity. For additional information, see the Elementary and Secondary Enrollment section A.1 in appendix A. Detail may not sum to totals because of rounding. Mean absolute percentage errors of selected education statistics can be found in table A-2, appendix A.

SOURCE: U.S. Department of Education, National Center for Education Statistics, Common Core of Data (CCD), "State Nonfiscal Survey of Public Elementary/Secondary Education," 1995-96 through 2008-09; and National Public Elementary and Secondary Enrollment by Race/Ethnicity Model, 1994-2008. (This table was prepared January 2011.) 
This page intentionally left blank. 
Table 6. Actual and projected numbers for enrollment in grades PK-12 in public elementary and secondary schools, by region and state: Fall 2002 through fall 2020

\begin{tabular}{|c|c|c|c|c|c|c|c|c|c|c|}
\hline \multirow[b]{3}{*}{ Region and state } & \multirow{2}{*}{\multicolumn{7}{|c|}{ [In thousands] }} & \multirow{2}{*}{\multicolumn{3}{|c|}{ Projected }} \\
\hline & & & & & & & & & & \\
\hline & 2002 & 2003 & 2004 & 2005 & 2006 & 2007 & 2008 & 2009 & 2010 & 2011 \\
\hline United States & 48,183 & 48,540 & 48,795 & 49,113 & 49,316 & 49,293 & 49,265 & 49,282 & 49,306 & 49,422 \\
\hline Northeast & 8,297 & 8,292 & 8,271 & 8,240 & 8,258 & 8,122 & 8,052 & 7,960 & 7,887 & 7,831 \\
\hline Connecticut & 570 & 577 & 577 & 575 & 575 & 571 & 567 & 561 & 556 & 552 \\
\hline Maine & 204 & 202 & 199 & 195 & 194 & 196 & 193 & 191 & 189 & 189 \\
\hline Massachusetts & 983 & 980 & 976 & 972 & 969 & 963 & 959 & 952 & 944 & 938 \\
\hline New Hampshire & 208 & 207 & 207 & 206 & 204 & 201 & 198 & 195 & 192 & 190 \\
\hline New Jersey & 1,367 & 1,381 & 1,393 & 1,396 & 1,389 & 1,382 & 1,381 & 1,375 & 1,374 & 1,373 \\
\hline New York & 2,888 & 2,865 & 2,836 & 2,816 & 2,810 & 2,765 & 2,741 & 2,700 & 2,667 & 2,641 \\
\hline Pennsylvania & 1,817 & 1,821 & 1,828 & 1,831 & 1,871 & 1,802 & 1,775 & 1,752 & 1,735 & 1,720 \\
\hline Rhode Island & 159 & 159 & 156 & 153 & 152 & 148 & 145 & 143 & 141 & 139 \\
\hline Vermont & 100 & 99 & 98 & 97 & 95 & 94 & 92 & 91 & 90 & 89 \\
\hline Midwest & 10,819 & 10,809 & 10,775 & 10,819 & 10,819 & 10,770 & 10,743 & 10,700 & 10,654 & 10,622 \\
\hline Illinois & 2,084 & 2,101 & 2,098 & 2,112 & 2,118 & 2,113 & 2,120 & 2,121 & 2,120 & 2,115 \\
\hline Indiana & 1,004 & 1,011 & 1,021 & 1,035 & 1,046 & 1,047 & 1,046 & 1,046 & 1,044 & 1,044 \\
\hline lowa & 482 & 481 & 478 & 483 & 483 & 485 & 488 & 487 & 486 & 487 \\
\hline Kansas & 471 & 470 & 469 & 468 & 470 & 468 & 471 & 472 & 474 & 476 \\
\hline Michigan & 1,785 & 1,758 & 1,751 & 1,742 & 1,723 & 1,693 & 1,660 & 1,631 & 1,605 & 1,581 \\
\hline Minnesota & 847 & 843 & 839 & 839 & 841 & 838 & 836 & 840 & 841 & 846 \\
\hline Missouri & 906 & 906 & 905 & 918 & 920 & 917 & 918 & 916 & 912 & 910 \\
\hline Nebraska & 285 & 286 & 286 & 287 & 288 & 291 & 293 & 294 & 296 & 298 \\
\hline North Dakota & 104 & 102 & 101 & 98 & 97 & 95 & 95 & 94 & 93 & 93 \\
\hline Ohio & 1,838 & 1,845 & 1,840 & 1,840 & 1,837 & 1,827 & 1,817 & 1,803 & 1,789 & 1,778 \\
\hline South Dakota & 130 & 126 & 123 & 122 & 121 & 122 & 127 & 128 & 129 & 130 \\
\hline Wisconsin & 881 & 880 & 865 & 875 & 877 & 875 & 874 & 869 & 866 & 865 \\
\hline South & 17,471 & 17,673 & 17,892 & 18,103 & 18,294 & 18,425 & 18,491 & 18,600 & 18,691 & 18,814 \\
\hline Alabama & 739 & 731 & 730 & 742 & 744 & 745 & 746 & 745 & 743 & 741 \\
\hline Arkansas & 451 & 455 & 463 & 474 & 476 & 479 & 479 & 481 & 482 & 484 \\
\hline Delaware & 116 & 118 & 119 & 121 & 122 & 123 & 125 & 126 & 127 & 128 \\
\hline District of Columbia & 76 & 78 & 77 & 77 & 73 & 78 & 69 & 72 & 72 & 72 \\
\hline Florida & 2,540 & 2,588 & 2,639 & 2,675 & 2,672 & 2,667 & 2,631 & 2,606 & 2,591 & 2,586 \\
\hline Georgia & 1,496 & 1,523 & 1,553 & 1,598 & 1,629 & 1,650 & 1,656 & 1,667 & 1,676 & 1,686 \\
\hline Kentucky & 661 & 663 & 675 & 680 & 683 & 666 & 670 & 671 & 671 & 671 \\
\hline Louisiana & 730 & 728 & 724 & 655 & 676 & 681 & 685 & 687 & 686 & 686 \\
\hline Maryland & 867 & 869 & 866 & 860 & 852 & 846 & 844 & 840 & 837 & 837 \\
\hline Mississippi & 493 & 494 & 495 & 495 & 495 & 494 & 492 & 491 & 489 & 487 \\
\hline North Carolina & 1,336 & 1,360 & 1,386 & 1,416 & 1,444 & 1,489 & 1,489 & 1,509 & 1,526 & 1,541 \\
\hline Oklahoma & 625 & 626 & 629 & 635 & 639 & 642 & 645 & 648 & 650 & 653 \\
\hline South Carolina & 694 & 699 & 704 & 702 & 708 & 712 & 718 & 722 & 722 & 725 \\
\hline Tennessee & 928 & 937 & 941 & 954 & 978 & 964 & 972 & 973 & 975 & 979 \\
\hline Texas & 4,260 & 4,332 & 4,405 & 4,525 & 4,600 & 4,675 & 4,752 & 4,839 & 4,920 & 5,011 \\
\hline Virginia & 1,177 & 1,192 & 1,205 & 1,214 & 1,220 & 1,231 & 1,236 & 1,239 & 1,241 & 1,245 \\
\hline West Virginia & 282 & 281 & 280 & 281 & 282 & 283 & 283 & 284 & 283 & 282 \\
\hline West & 11,596 & 11,766 & 11,857 & 11,951 & 11,945 & 11,976 & 11,979 & 12,022 & 12,073 & 12,155 \\
\hline Alaska & 134 & 134 & 133 & 133 & 133 & 131 & 131 & 131 & 132 & 133 \\
\hline Arizona & 938 & 1,012 & 1,043 & 1,094 & 1,068 & 1,087 & 1,088 & 1,100 & 1,116 & 1,136 \\
\hline California & 6,354 & 6,414 & 6,442 & 6,437 & 6,407 & 6,343 & 6,323 & 6,319 & 6,318 & 6,329 \\
\hline Colorado & 752 & 758 & 766 & 780 & 794 & 802 & 818 & 831 & 843 & 856 \\
\hline Hawaii & 184 & 184 & 183 & 183 & 181 & 180 & 179 & 179 & 180 & 180 \\
\hline Idaho & 249 & 252 & 256 & 262 & 267 & 272 & 275 & 279 & 282 & 286 \\
\hline Montana & 150 & 148 & 147 & 145 & 144 & 143 & 142 & 141 & 140 & 141 \\
\hline Nevada & 369 & 385 & 400 & 412 & 425 & 429 & 433 & 442 & 449 & 458 \\
\hline New Mexico & 320 & 323 & 326 & 327 & 328 & 329 & 330 & 331 & 334 & 336 \\
\hline Oregon & 554 & 551 & 553 & 552 & 563 & 566 & 575 & 574 & 576 & 580 \\
\hline Utah & 489 & 496 & 504 & 508 & 523 & 576 & 560 & 563 & 567 & 572 \\
\hline Washington & 1,015 & 1,021 & 1,020 & 1,032 & 1,027 & 1,030 & 1,037 & 1,043 & 1,048 & 1,057 \\
\hline Wyoming & 88 & 87 & 85 & 84 & 85 & 86 & 87 & 88 & 90 & 91 \\
\hline
\end{tabular}

See notes at end of table. 
Table 6. Actual and projected numbers for enrollment in grades PK-12 in public elementary and secondary schools, by region and state: Fall 2002 through fall 2020-Continued

[In thousands]

\begin{tabular}{|c|c|c|c|c|c|c|c|c|c|}
\hline \multirow[b]{2}{*}{ Region and state } & \multicolumn{9}{|c|}{ Projected-Continued } \\
\hline & 2012 & 2013 & 2014 & 2015 & 2016 & 2017 & 2018 & 2019 & 2020 \\
\hline United States & 49,642 & 49,914 & 50,268 & 50,659 & 51,038 & 51,430 & 51,803 & 52,204 & 52,666 \\
\hline Northeast & 7,790 & 7,762 & 7,752 & 7,753 & 7,758 & 7,770 & 7,784 & 7,805 & 7,836 \\
\hline Connecticut & 549 & 547 & 545 & 545 & 544 & 545 & 547 & 549 & 552 \\
\hline Maine & 188 & 188 & 188 & 189 & 190 & 191 & 192 & 193 & 195 \\
\hline Massachusetts & 933 & 928 & 924 & 922 & 919 & 918 & 917 & 917 & 919 \\
\hline New Hampshire & 188 & 187 & 187 & 187 & 187 & 188 & 189 & 191 & 193 \\
\hline New Jersey & 1,373 & 1,373 & 1,375 & 1,377 & 1,380 & 1,382 & 1,386 & 1,390 & 1,396 \\
\hline New York & 2,621 & 2,606 & 2,598 & 2,594 & 2,592 & 2,593 & 2,593 & 2,597 & 2,602 \\
\hline Pennsylvania & 1,711 & 1,706 & 1,706 & 1,710 & 1,714 & 1,718 & 1,723 & 1,729 & 1,739 \\
\hline Rhode Island & 138 & 138 & 138 & 138 & 139 & 140 & 142 & 143 & 144 \\
\hline Vermont & 89 & 90 & 90 & 91 & 92 & 93 & 95 & 96 & 98 \\
\hline Midwest & 10,619 & 10,631 & 10,662 & 10,699 & 10,730 & 10,760 & 10,783 & 10,805 & 10,846 \\
\hline Illinois & 2,113 & 2,113 & 2,118 & 2,123 & 2,128 & 2,131 & 2,133 & 2,136 & 2,143 \\
\hline Indiana & 1,044 & 1,045 & 1,046 & 1,049 & 1,051 & 1,053 & 1,055 & 1,055 & 1,059 \\
\hline lowa & 489 & 491 & 494 & 496 & 498 & 499 & 499 & 499 & 499 \\
\hline Kansas & 480 & 483 & 487 & 491 & 494 & 496 & 497 & 499 & 500 \\
\hline Michigan & 1,570 & 1,560 & 1,555 & 1,552 & 1,549 & 1,548 & 1,547 & 1,547 & 1,552 \\
\hline Minnesota & 853 & 862 & 874 & 886 & 899 & 912 & 924 & 936 & 950 \\
\hline Missouri & 911 & 913 & 917 & 920 & 922 & 925 & 927 & 929 & 932 \\
\hline Nebraska & 300 & 303 & 305 & 308 & 310 & 313 & 314 & 315 & 316 \\
\hline North Dakota & 93 & 93 & 93 & 93 & 93 & 93 & 94 & 94 & 94 \\
\hline Ohio & 1,770 & 1,766 & 1,765 & 1,765 & 1,762 & 1,760 & 1,757 & 1,753 & 1,752 \\
\hline South Dakota & 131 & 132 & 133 & 134 & 135 & 136 & 137 & 138 & 139 \\
\hline Wisconsin & 866 & 870 & 876 & 882 & 888 & 894 & 899 & 904 & 910 \\
\hline South & 18,977 & 19,146 & 19,339 & 19,531 & 19,709 & 19,883 & 20,043 & 20,211 & 20,399 \\
\hline Alabama & 740 & 739 & 740 & 739 & 737 & 734 & 731 & 729 & 728 \\
\hline Arkansas & 486 & 488 & 490 & 492 & 493 & 493 & 494 & 494 & 495 \\
\hline Delaware & 130 & 131 & 133 & 135 & 137 & 138 & 139 & 140 & 142 \\
\hline District of Columbia & 72 & 72 & 73 & 73 & 74 & 75 & 75 & 76 & 76 \\
\hline Florida & 2,595 & 2,602 & 2,617 & 2,634 & 2,657 & 2,682 & 2,712 & 2,746 & 2,788 \\
\hline Georgia & 1,700 & 1,716 & 1,733 & 1,751 & 1,766 & 1,780 & 1,794 & 1,808 & 1,826 \\
\hline Kentucky & 672 & 672 & 673 & 674 & 673 & 672 & 671 & 670 & 669 \\
\hline Louisiana & 686 & 685 & 683 & 684 & 682 & 679 & 675 & 671 & 668 \\
\hline Maryland & 838 & 842 & 850 & 859 & 870 & 882 & 895 & 909 & 921 \\
\hline Mississippi & 486 & 484 & 483 & 482 & 481 & 478 & 474 & 471 & 468 \\
\hline North Carolina & 1,556 & 1,576 & 1,598 & 1,619 & 1,638 & 1,656 & 1,673 & 1,690 & 1,713 \\
\hline Oklahoma & 656 & 660 & 664 & 668 & 669 & 671 & 671 & 672 & 672 \\
\hline South Carolina & 728 & 733 & 738 & 743 & 746 & 749 & 750 & 751 & 754 \\
\hline Tennessee & 985 & 991 & 998 & 1,004 & 1,010 & 1,014 & 1,019 & 1,024 & 1,031 \\
\hline Texas & 5,113 & 5,211 & 5,313 & 5,408 & 5,502 & 5,592 & 5,673 & 5,751 & 5,830 \\
\hline Virginia & 1,252 & 1,261 & 1,273 & 1,287 & 1,299 & 1,312 & 1,325 & 1,338 & 1,352 \\
\hline West Virginia & 282 & 281 & 280 & 279 & 277 & 276 & 273 & 270 & 267 \\
\hline West & 12,256 & 12,374 & 12,515 & 12,676 & 12,842 & 13,017 & 13,194 & 13,383 & 13,585 \\
\hline Alaska & 135 & 138 & 141 & 144 & 148 & 152 & 155 & 159 & 163 \\
\hline Arizona & 1,160 & 1,184 & 1,209 & 1,236 & 1,263 & 1,289 & 1,316 & 1,343 & 1,373 \\
\hline California & 6,349 & 6,379 & 6,425 & 6,488 & 6,558 & 6,640 & 6,721 & 6,812 & 6,908 \\
\hline Colorado & 869 & 883 & 897 & 911 & 924 & 936 & 946 & 956 & 966 \\
\hline Hawaii & 180 & 182 & 182 & 183 & 185 & 186 & 187 & 188 & 188 \\
\hline Idaho & 291 & 296 & 300 & 306 & 311 & 315 & 319 & 323 & 327 \\
\hline Montana & 141 & 142 & 143 & 144 & 145 & 146 & 147 & 148 & 149 \\
\hline Nevada & 467 & 476 & 486 & 497 & 508 & 519 & 530 & 542 & 556 \\
\hline New Mexico & 339 & 344 & 348 & 353 & 357 & 360 & 363 & 365 & 367 \\
\hline Oregon & 585 & 592 & 598 & 606 & 614 & 622 & 630 & 640 & 650 \\
\hline Utah & 578 & 583 & 589 & 594 & 599 & 604 & 609 & 616 & 625 \\
\hline Washington & 1,069 & 1,082 & 1,098 & 1,116 & 1,133 & 1,151 & 1,170 & 1,191 & 1,213 \\
\hline Wyoming & 93 & 95 & 96 & 97 & 98 & 99 & 99 & 99 & 99 \\
\hline
\end{tabular}

NOTE: PK=prekindergarten. Some data have been revised from previously published figures. Detail may not sum to totals because of rounding. Mean absolute percentage errors of PK-12 enrollment in public elementary and secondary schools by state and region can be found in table A-7, appendix A. SOURCE: U.S. Department of Education, National Center for Education Statistics, Common Core of Data (CCD), "State Nonfiscal Survey of Public Elementary/Secondary Education," 2002-03 through 2008-09; and State Public Elementary and Secondary Enrollment Model, 1980-2008. (This table was prepared January 2011.) 
Table 7. Actual and projected percentage changes in grades PK-12 enrollment in public elementary and secondary schools, by region and state: Selected years, fall 2002 through fall 2020

\begin{tabular}{|c|c|c|c|c|}
\hline \multirow[b]{2}{*}{ Region and state } & \multirow[b]{2}{*}{ Actual 2002-2008 } & \multicolumn{3}{|c|}{ Projected } \\
\hline & & 2008-2014 & 2014-2020 & 2008-2020 \\
\hline United States & 2.2 & 2.0 & 4.8 & 6.9 \\
\hline Northeast & -2.9 & -3.7 & 1.1 & -2.7 \\
\hline Connecticut & -0.5 & -3.9 & 1.3 & -2.6 \\
\hline Maine & -5.6 & -2.3 & 3.2 & 0.8 \\
\hline Massachusetts & -2.4 & -3.6 & -0.6 & -4.2 \\
\hline New Hampshire & -4.7 & -5.6 & 3.1 & -2.7 \\
\hline New Jersey & 1.0 & -0.5 & 1.5 & 1.0 \\
\hline New York & -5.1 & -5.2 & 0.2 & -5.1 \\
\hline Pennsylvania & -2.3 & -3.9 & 1.9 & -2.1 \\
\hline Rhode Island & -8.7 & -5.4 & 4.8 & -0.8 \\
\hline Vermont & -7.5 & -2.3 & 8.0 & 5.6 \\
\hline Midwest & -0.7 & -0.8 & 1.7 & 1.0 \\
\hline Illinois & 1.7 & -0.1 & 1.2 & 1.1 \\
\hline Indiana & 4.2 & \# & 1.2 & 1.2 \\
\hline lowa & 1.1 & 1.3 & 1.2 & 2.4 \\
\hline Kansas & $\#$ & 3.3 & 2.7 & 6.2 \\
\hline Michigan & -7.0 & -6.3 & -0.2 & -6.5 \\
\hline Minnesota & -1.3 & 4.5 & 8.7 & 13.6 \\
\hline Missouri & 1.3 & -0.1 & 1.6 & 1.5 \\
\hline Nebraska & 2.5 & 4.3 & 3.5 & 7.9 \\
\hline North Dakota & -9.1 & -2.1 & 1.7 & -0.5 \\
\hline Ohio & -1.1 & -2.9 & -0.7 & -3.6 \\
\hline South Dakota & -2.5 & 5.0 & 4.2 & 9.4 \\
\hline Wisconsin & -0.8 & 0.2 & 3.9 & 4.1 \\
\hline South & 5.8 & 4.6 & 5.5 & 10.3 \\
\hline Alabama & 0.9 & -0.8 & -1.6 & -2.4 \\
\hline Arkansas & 6.2 & 2.3 & 0.9 & 3.3 \\
\hline Delaware & 7.8 & 6.1 & 6.4 & 12.9 \\
\hline District of Columbia & -9.8 & 5.9 & 4.2 & 10.3 \\
\hline Florida & 3.6 & -0.5 & 6.5 & 5.9 \\
\hline Georgia & 10.7 & 4.7 & 5.3 & 10.3 \\
\hline Kentucky & 1.4 & 0.5 & -0.7 & -0.2 \\
\hline Louisiana & -6.2 & -0.2 & -2.2 & -2.4 \\
\hline Maryland & -2.6 & 0.7 & 8.4 & 9.2 \\
\hline Mississippi & -0.1 & -1.7 & -3.2 & -4.9 \\
\hline North Carolina & 11.4 & 7.4 & 7.2 & 15.1 \\
\hline Oklahoma & 3.3 & 3.0 & 1.2 & 4.2 \\
\hline South Carolina & 3.4 & 2.8 & 2.1 & 5.0 \\
\hline Tennessee & 4.8 & 2.7 & 3.3 & 6.1 \\
\hline Texas & 11.6 & 11.8 & 9.7 & 22.7 \\
\hline Virginia & 5.0 & 3.0 & 6.2 & 9.4 \\
\hline West Virginia & 0.1 & -1.0 & -4.6 & -5.5 \\
\hline West & 3.3 & 4.5 & 8.6 & 13.4 \\
\hline Alaska & -2.8 & 7.9 & 15.7 & 24.8 \\
\hline Arizona & 16.0 & 11.2 & 13.5 & 26.2 \\
\hline California & -0.5 & 1.6 & 7.5 & 9.3 \\
\hline Colorado & 8.9 & 9.6 & 7.7 & 18.0 \\
\hline Hawaii & -2.4 & 1.6 & 3.3 & 5.0 \\
\hline Idaho & 10.7 & 9.2 & 8.7 & 18.7 \\
\hline Montana & -5.4 & 0.9 & 3.8 & 4.7 \\
\hline Nevada & 17.3 & 12.3 & 14.3 & 28.4 \\
\hline New Mexico & 3.1 & 5.5 & 5.5 & 11.2 \\
\hline Oregon & 3.8 & 4.0 & 8.6 & 13.0 \\
\hline Utah & 14.4 & 5.3 & 6.1 & 11.7 \\
\hline Washington & 2.2 & 5.9 & 10.5 & 17.0 \\
\hline Wyoming & -1.1 & 10.2 & 3.3 & 13.8 \\
\hline
\end{tabular}

\# Rounds to zero.

NOTE: PK=prekindergarten. Calculations are based on unrounded numbers. Mean absolute percentage errors of PK-12 enrollment in public elementary and secondary schools by state and region can be found in table A-7, appendix $A$.

SOURCE: U.S. Department of Education, National Center for Education Statistics, Common Core of Data (CCD), "State Nonfiscal Survey of Public Elementary/Secondary Education," selected years, 2002-03 through 2008-09; and State Public Elementary and Secondary Enrollment Model, 1980-2008. (This table was prepared January 2011.) 
Table 8. Actual and projected numbers for enrollment in grades PK-8 in public elementary and secondary schools, by region and state: Fall 2002 through fall 2020

\begin{tabular}{|c|c|c|c|c|c|c|c|c|c|c|}
\hline \multirow[b]{2}{*}{ Region and state } & \multicolumn{7}{|c|}{ Actual } & \multicolumn{3}{|c|}{ Projected } \\
\hline & 2002 & 2003 & 2004 & 2005 & 2006 & 2007 & 2008 & 2009 & 2010 & 2011 \\
\hline United States & 34,114 & 34,201 & 34,178 & 34,204 & 34,235 & 34,205 & 34,285 & 34,440 & 34,637 & 34,892 \\
\hline Northeast & 5,810 & 5,752 & 5,689 & 5,623 & 5,574 & 5,504 & 5,476 & 5,435 & 5,413 & 5,403 \\
\hline Connecticut & 406 & 408 & 404 & 400 & 398 & 394 & 392 & 389 & 387 & 385 \\
\hline Maine & 142 & 139 & 136 & 133 & 132 & 131 & 129 & 129 & 129 & 129 \\
\hline Massachusetts & 701 & 692 & 682 & 675 & 671 & 667 & 667 & 663 & 660 & 657 \\
\hline New Hampshire & 144 & 142 & 140 & 139 & 136 & 134 & 133 & 131 & 130 & 129 \\
\hline New Jersey & 979 & 978 & 976 & 971 & 963 & 954 & 957 & 954 & 955 & 956 \\
\hline New York & 2,016 & 1,978 & 1,943 & 1,909 & 1,887 & 1,856 & 1,843 & 1,823 & 1,812 & 1,807 \\
\hline Pennsylvania & 1,242 & 1,236 & 1,235 & 1,228 & 1,220 & 1,205 & 1,194 & 1,186 & 1,182 & 1,182 \\
\hline Rhode Island & 113 & 111 & 107 & 104 & 102 & 99 & 98 & 97 & 97 & 96 \\
\hline Vermont & 68 & 67 & 66 & 65 & 64 & 63 & 62 & 62 & 62 & 62 \\
\hline Midwest & 7,535 & 7,502 & 7,439 & 7,425 & 7,405 & 7,359 & 7,374 & 7,383 & 7,393 & 7,414 \\
\hline Illinois & 1,488 & 1,493 & 1,484 & 1,480 & 1,478 & 1,473 & 1,479 & 1,483 & 1,484 & 1,488 \\
\hline Indiana & 714 & 717 & 720 & 724 & 730 & 730 & 730 & 731 & 731 & 732 \\
\hline lowa & 326 & 327 & 324 & 326 & 326 & 330 & 336 & 338 & 340 & 342 \\
\hline Kansas & 322 & 322 & 321 & 321 & 326 & 327 & 331 & 333 & 336 & 340 \\
\hline Michigan & 1,254 & 1,229 & 1,212 & 1,191 & 1,171 & 1,137 & 1,119 & 1,106 & 1,098 & 1,091 \\
\hline Minnesota & 568 & 564 & 558 & 558 & 558 & 558 & 560 & 569 & 576 & 583 \\
\hline Missouri & 635 & 632 & 629 & 635 & 634 & 632 & 635 & 638 & 640 & 642 \\
\hline Nebraska & 195 & 195 & 195 & 195 & 196 & 200 & 203 & 206 & 209 & 211 \\
\hline North Dakota & 69 & 68 & 67 & 66 & 64 & 63 & 64 & 64 & 64 & 64 \\
\hline Ohio & 1,284 & 1,278 & 1,267 & 1,261 & 1,253 & 1,241 & 1,239 & 1,236 & 1,234 & 1,233 \\
\hline South Dakota & 89 & 86 & 84 & 84 & 83 & 83 & 88 & 88 & 89 & 90 \\
\hline Wisconsin & 592 & 590 & 578 & 584 & 585 & 585 & 590 & 591 & 593 & 597 \\
\hline South & 12,573 & 12,675 & 12,780 & 12,882 & 12,990 & 13,086 & 13,167 & 13,299 & 13,431 & 13,573 \\
\hline Alabama & 533 & 525 & 522 & 529 & 529 & 527 & 528 & 529 & 530 & 530 \\
\hline Arkansas & 319 & 322 & 328 & 336 & 337 & 340 & 342 & 345 & 347 & 349 \\
\hline Delaware & 82 & 83 & 84 & 85 & 85 & 85 & 87 & 88 & 89 & 91 \\
\hline District of Columbia & 59 & 59 & 57 & 56 & 52 & 56 & 51 & 54 & 55 & 55 \\
\hline Florida & 1,809 & 1,832 & 1,858 & 1,873 & 1,867 & 1,856 & 1,849 & 1,840 & 1,840 & 1,843 \\
\hline Georgia & 1,089 & 1,103 & 1,118 & 1,145 & 1,167 & 1,179 & 1,186 & 1,199 & 1,210 & 1,223 \\
\hline Kentucky & 477 & 478 & 486 & 487 & 487 & 469 & 472 & 475 & 477 & 479 \\
\hline Louisiana & 537 & 536 & 534 & 482 & 492 & 500 & 504 & 507 & 506 & 509 \\
\hline Maryland & 610 & 606 & 597 & 589 & 579 & 576 & 576 & 575 & 578 & 582 \\
\hline Mississippi & 360 & 361 & 361 & 358 & 356 & 354 & 352 & 352 & 352 & 353 \\
\hline North Carolina & 964 & 974 & 986 & 1,003 & 1,027 & 1,072 & 1,059 & 1,074 & 1,090 & 1,105 \\
\hline Oklahoma & 449 & 450 & 453 & 457 & 460 & 463 & 468 & 472 & 476 & 480 \\
\hline South Carolina & 500 & 501 & 504 & 498 & 501 & 505 & 508 & 513 & 517 & 521 \\
\hline Tennessee & 673 & 675 & 671 & 677 & 692 & 682 & 685 & 689 & 694 & 698 \\
\hline Texas & 3,080 & 3,133 & 3,184 & 3,268 & 3,320 & 3,375 & 3,447 & 3,524 & 3,601 & 3,676 \\
\hline Virginia & 832 & 837 & 840 & 841 & 842 & 850 & 855 & 862 & 869 & 878 \\
\hline West Virginia & 200 & 199 & 198 & 197 & 198 & 199 & 199 & 201 & 202 & 202 \\
\hline West & 8,197 & 8,272 & 8,270 & 8,274 & 8,267 & 8,256 & 8,269 & 8,324 & 8,400 & 8,501 \\
\hline Alaska & 94 & 94 & 92 & 91 & 90 & 89 & 89 & 90 & 92 & 94 \\
\hline Arizona & 660 & 704 & 722 & 740 & 760 & 771 & 772 & 788 & 805 & 822 \\
\hline California & 4,525 & 4,540 & 4,507 & 4,466 & 4,410 & 4,329 & 4,306 & 4,301 & 4,315 & 4,347 \\
\hline Colorado & 534 & 536 & 541 & 550 & 559 & 566 & 580 & 591 & 602 & 613 \\
\hline Hawaii & 131 & 130 & 129 & 127 & 126 & 126 & 126 & 127 & 128 & 129 \\
\hline Idaho & 173 & 175 & 178 & 183 & 187 & 191 & 194 & 197 & 200 & 204 \\
\hline Montana & 101 & 100 & 99 & 98 & 97 & 96 & 97 & 97 & 98 & 98 \\
\hline Nevada & 271 & 281 & 289 & 296 & 303 & 308 & 308 & 314 & 320 & 327 \\
\hline New Mexico & 224 & 226 & 228 & 230 & 230 & 230 & 231 & 234 & 238 & 241 \\
\hline Oregon & 382 & 378 & 377 & 380 & 381 & 384 & 395 & 397 & 400 & 404 \\
\hline Utah & 343 & 349 & 355 & 358 & 371 & 410 & 404 & 412 & 418 & 424 \\
\hline Washington & 697 & 699 & 695 & 699 & 695 & 697 & 705 & 712 & 721 & 731 \\
\hline Wyoming & 60 & 60 & 57 & 57 & 58 & 59 & 61 & 62 & 64 & 65 \\
\hline
\end{tabular}

See notes at end of table. 
Table 8. Actual and projected numbers for enrollment in grades PK-8 in public elementary and secondary schools, by region and state: Fall 2002 through fall 2020-Continued

[In thousands]

\begin{tabular}{|c|c|c|c|c|c|c|c|c|c|}
\hline \multirow[b]{2}{*}{ Region and state } & \multicolumn{9}{|c|}{ Projected-Continued } \\
\hline & 2012 & 2013 & 2014 & 2015 & 2016 & 2017 & 2018 & 2019 & 2020 \\
\hline United States & 35,129 & 35,368 & 35,579 & 35,829 & 36,161 & 36,491 & 36,803 & 37,121 & 37,444 \\
\hline Northeast & 5,395 & 5,391 & 5,389 & 5,395 & 5,415 & 5,435 & 5,455 & 5,479 & 5,505 \\
\hline Connecticut & 383 & 382 & 382 & 382 & 384 & 386 & 388 & 391 & 395 \\
\hline Maine & 129 & 130 & 131 & 132 & 133 & 134 & 135 & 136 & 136 \\
\hline Massachusetts & 653 & 651 & 648 & 646 & 645 & 645 & 646 & 647 & 650 \\
\hline New Hampshire & 128 & 128 & 129 & 129 & 130 & 132 & 134 & 136 & 138 \\
\hline New Jersey & 957 & 957 & 958 & 959 & 962 & 964 & 967 & 970 & 975 \\
\hline New York & 1,802 & 1,799 & 1,795 & 1,794 & 1,797 & 1,801 & 1,804 & 1,809 & 1,814 \\
\hline Pennsylvania & 1,183 & 1,183 & 1,184 & 1,188 & 1,196 & 1,203 & 1,210 & 1,217 & 1,223 \\
\hline Rhode Island & 96 & 98 & 99 & 99 & 100 & 101 & 102 & 103 & 104 \\
\hline Vermont & 63 & 64 & 65 & 66 & 67 & 68 & 69 & 70 & 71 \\
\hline Midwest & 7,430 & 7,446 & 7,455 & 7,468 & 7,503 & 7,538 & 7,568 & 7,596 & 7,622 \\
\hline Illinois & 1,491 & 1,492 & 1,491 & 1,491 & 1,498 & 1,503 & 1,507 & 1,511 & 1,515 \\
\hline Indiana & 734 & 735 & 734 & 733 & 735 & 738 & 740 & 743 & 747 \\
\hline lowa & 344 & 345 & 346 & 347 & 348 & 348 & 347 & 347 & 346 \\
\hline Kansas & 342 & 344 & 345 & 346 & 348 & 349 & 350 & 351 & 351 \\
\hline Michigan & 1,085 & 1,081 & 1,078 & 1,078 & 1,082 & 1,089 & 1,095 & 1,100 & 1,105 \\
\hline Minnesota & 591 & 599 & 607 & 615 & 626 & 635 & 644 & 653 & 662 \\
\hline Missouri & 643 & 645 & 646 & 647 & 649 & 652 & 654 & 656 & 658 \\
\hline Nebraska & 214 & 216 & 217 & 218 & 219 & 219 & 219 & 219 & 219 \\
\hline North Dakota & 64 & 65 & 65 & 65 & 66 & 66 & 66 & 66 & 66 \\
\hline Ohio & 1,231 & 1,228 & 1,224 & 1,221 & 1,221 & 1,222 & 1,222 & 1,222 & 1,221 \\
\hline South Dakota & 91 & 92 & 93 & 94 & 95 & 95 & 95 & 95 & 95 \\
\hline Wisconsin & 601 & 605 & 608 & 612 & 618 & 623 & 628 & 632 & 636 \\
\hline South & 13,699 & 13,814 & 13,906 & 14,008 & 14,138 & 14,274 & 14,402 & 14,535 & 14,672 \\
\hline Alabama & 529 & 526 & 523 & 520 & 519 & 519 & 518 & 517 & 517 \\
\hline Arkansas & 350 & 350 & 350 & 349 & 349 & 349 & 350 & 350 & 351 \\
\hline Delaware & 92 & 93 & 94 & 95 & 96 & 97 & 97 & 98 & 99 \\
\hline District of Columbia & 56 & 57 & 58 & 59 & 60 & 61 & 60 & 59 & 59 \\
\hline Florida & 1,852 & 1,863 & 1,876 & 1,894 & 1,921 & 1,954 & 1,986 & 2,018 & 2,051 \\
\hline Georgia & 1,233 & 1,242 & 1,250 & 1,257 & 1,268 & 1,281 & 1,294 & 1,307 & 1,321 \\
\hline Kentucky & 478 & 478 & 477 & 476 & 474 & 473 & 471 & 470 & 469 \\
\hline Louisiana & 508 & 506 & 503 & 500 & 498 & 496 & 493 & 491 & 488 \\
\hline Maryland & 587 & 595 & 603 & 613 & 621 & 631 & 641 & 652 & 662 \\
\hline Mississippi & 353 & 351 & 348 & 345 & 344 & 341 & 338 & 336 & 333 \\
\hline North Carolina & 1,117 & 1,127 & 1,135 & 1,144 & 1,156 & 1,172 & 1,187 & 1,205 & 1,224 \\
\hline Oklahoma & 482 & 483 & 483 & 483 & 483 & 483 & 483 & 484 & 484 \\
\hline South Carolina & 524 & 525 & 525 & 526 & 528 & 530 & 532 & 534 & 536 \\
\hline Tennessee & 701 & 703 & 705 & 707 & 710 & 715 & 720 & 726 & 733 \\
\hline Texas & 3,750 & 3,819 & 3,878 & 3,936 & 3,997 & 4,052 & 4,103 & 4,153 & 4,202 \\
\hline Virginia & 885 & 893 & 900 & 908 & 917 & 928 & 938 & 949 & 960 \\
\hline West Virginia & 201 & 201 & 199 & 197 & 195 & 193 & 190 & 187 & 185 \\
\hline West & 8,606 & 8,717 & 8,829 & 8,958 & 9,104 & 9,244 & 9,378 & 9,512 & 9,645 \\
\hline Alaska & 97 & 99 & 102 & 104 & 107 & 111 & 113 & 116 & 119 \\
\hline Arizona & 840 & 857 & 874 & 892 & 912 & 932 & 953 & 974 & 996 \\
\hline California & 4,386 & 4,435 & 4,485 & 4,548 & 4,619 & 4,686 & 4,750 & 4,813 & 4,875 \\
\hline Colorado & 623 & 632 & 639 & 646 & 652 & 658 & 664 & 671 & 677 \\
\hline Hawaii & 130 & 132 & 132 & 134 & 135 & 135 & 135 & 135 & 135 \\
\hline Idaho & 208 & 211 & 214 & 217 & 221 & 224 & 227 & 229 & 231 \\
\hline Montana & 99 & 100 & 101 & 102 & 103 & 104 & 104 & 104 & 104 \\
\hline Nevada & 334 & 340 & 345 & 352 & 360 & 369 & 380 & 390 & 402 \\
\hline New Mexico & 245 & 248 & 252 & 254 & 257 & 259 & 260 & 261 & 261 \\
\hline Oregon & 408 & 413 & 418 & 425 & 432 & 440 & 447 & 455 & 462 \\
\hline Utah & 428 & 432 & 437 & 442 & 448 & 452 & 455 & 459 & 463 \\
\hline Washington & 740 & 751 & 762 & 774 & 789 & 805 & 821 & 837 & 854 \\
\hline Wyoming & 67 & 67 & 68 & 69 & 69 & 69 & 68 & 68 & 67 \\
\hline
\end{tabular}

NOTE: PK=prekindergarten. Some data have been revised from previously published figures. Detail may not sum to totals because of rounding. Mean absolute percentage errors of PK-8 enrollment in public elementary and secondary schools by state and region can be found in table A-8, appendix A. SOURCE: U.S. Department of Education, National Center for Education Statistics, Common Core of Data (CCD), "State Nonfiscal Survey of Public Elementary/Secondary Education," 2002-03 through 2008-09; and State Public Elementary and Secondary Enrollment Model, 1980-2008. (This table was prepared January 2011.)

42 Reference Tables 
Table 9. Actual and projected percentage changes in grades PK-8 enrollment in public elementary and secondary schools, by region and state: Selected years, fall 2002 through fall 2020

\begin{tabular}{|c|c|c|c|c|}
\hline \multirow[b]{2}{*}{ Region and state } & \multirow[b]{2}{*}{ Actual 2002-2008 } & \multicolumn{3}{|c|}{ Projected } \\
\hline & & 2008-2014 & 2014-2020 & 2008-2020 \\
\hline United States & 0.5 & 3.8 & 5.2 & 9.2 \\
\hline Northeast & -5.7 & -1.6 & 2.2 & 0.5 \\
\hline Connecticut & -3.4 & -2.7 & 3.4 & 0.6 \\
\hline Maine & -8.8 & 1.1 & 4.2 & 5.3 \\
\hline Massachusetts & -4.9 & -2.8 & 0.3 & -2.5 \\
\hline New Hampshire & -7.4 & -3.3 & 7.0 & 3.4 \\
\hline New Jersey & -2.2 & 0.1 & 1.7 & 1.9 \\
\hline New York & -8.6 & -2.6 & 1.1 & -1.6 \\
\hline Pennsylvania & -3.8 & -0.9 & 3.3 & 2.4 \\
\hline Rhode Island & -12.9 & 0.8 & 5.4 & 6.2 \\
\hline Vermont & -8.2 & 3.5 & 10.1 & 13.9 \\
\hline Midwest & -2.1 & 1.1 & 2.2 & 3.4 \\
\hline Illinois & -0.6 & 0.8 & 1.7 & 2.5 \\
\hline Indiana & 2.2 & 0.6 & 1.7 & 2.3 \\
\hline lowa & 3.0 & 3.2 & -0.1 & 3.1 \\
\hline Kansas & 2.9 & 4.3 & 1.8 & 6.1 \\
\hline Michigan & -10.8 & -3.6 & 2.5 & -1.2 \\
\hline Minnesota & -1.3 & 8.4 & 9.0 & 18.2 \\
\hline Missouri & 0.1 & 1.6 & 1.9 & 3.6 \\
\hline Nebraska & 4.0 & 7.0 & 0.9 & 7.9 \\
\hline North Dakota & -7.4 & 1.8 & 0.8 & 2.6 \\
\hline Ohio & -3.5 & -1.2 & -0.2 & -1.5 \\
\hline South Dakota & -2.0 & 6.1 & 2.3 & 8.5 \\
\hline Wisconsin & -0.4 & 3.1 & 4.7 & 7.9 \\
\hline South & 4.7 & 5.6 & 5.5 & 11.4 \\
\hline Alabama & -1.0 & -1.0 & -1.2 & -2.2 \\
\hline Arkansas & 7.1 & 2.5 & 0.3 & 2.8 \\
\hline Delaware & 5.6 & 8.1 & 5.4 & 13.9 \\
\hline District of Columbia & -13.6 & 14.1 & 1.2 & 15.5 \\
\hline Florida & 2.2 & 1.4 & 9.4 & 10.9 \\
\hline Georgia & 8.9 & 5.4 & 5.8 & 11.4 \\
\hline Kentucky & -1.0 & 1.0 & -1.6 & -0.7 \\
\hline Louisiana & -6.1 & -0.3 & -2.9 & -3.2 \\
\hline Maryland & -5.5 & 4.6 & 9.8 & 14.8 \\
\hline Mississippi & -2.3 & -1.1 & -4.4 & -5.4 \\
\hline North Carolina & 9.9 & 7.2 & 7.8 & 15.5 \\
\hline Oklahoma & 4.2 & 3.2 & 0.2 & 3.4 \\
\hline South Carolina & 1.4 & 3.5 & 2.1 & 5.7 \\
\hline Tennessee & 1.7 & 2.9 & 4.0 & 7.0 \\
\hline Texas & 11.9 & 12.5 & 8.4 & 21.9 \\
\hline Virginia & 2.8 & 5.3 & 6.6 & 12.3 \\
\hline West Virginia & -0.3 & -0.3 & -7.2 & -7.4 \\
\hline West & 0.9 & 6.8 & 9.2 & 16.6 \\
\hline Alaska & -5.4 & 13.9 & 16.6 & 32.8 \\
\hline Arizona & 16.9 & 13.2 & 13.9 & 29.0 \\
\hline California & -4.8 & 4.1 & 8.7 & 13.2 \\
\hline Colorado & 8.6 & 10.1 & 6.0 & 16.7 \\
\hline Hawaii & -3.8 & 5.2 & 1.8 & 7.0 \\
\hline Idaho & 11.7 & 10.8 & 7.8 & 19.4 \\
\hline Montana & -4.3 & 4.4 & 3.3 & 7.8 \\
\hline Nevada & 13.8 & 12.0 & 16.3 & 30.3 \\
\hline New Mexico & 3.1 & 8.7 & 3.8 & 12.8 \\
\hline Oregon & 3.5 & 5.8 & 10.4 & 16.8 \\
\hline Utah & 18.1 & 8.0 & 6.0 & 14.5 \\
\hline Washington & 1.1 & 8.1 & 12.1 & 21.1 \\
\hline Wyoming & 1.2 & 12.5 & -1.9 & 10.4 \\
\hline
\end{tabular}

NOTE: PK=prekindergarten. Calculations are based on unrounded numbers. Mean absolute percentage errors of PK- 8 enrollment in public elementary and secondary schools by state and region can be found in table A-8, appendix A.

SOURCE: U.S. Department of Education, National Center for Education Statistics, Common Core of Data (CCD), "State Nonfiscal Survey of Public Elementary/Secondary Education," selected years, 2002-03 through 2008-09; and State Public Elementary and Secondary Enrollment Model, 1980-2008. (This table was prepared January 2011.) 
Table 10. Actual and projected numbers for enrollment in grades 9-12 in public schools, by region and state: Fall 2002 through fall 2020

\begin{tabular}{|c|c|c|c|c|c|c|c|c|c|c|}
\hline \multirow[b]{3}{*}{ Region and state } & \multirow{2}{*}{\multicolumn{7}{|c|}{$\begin{array}{c}\text { [In thousands] } \\
\text { Actual }\end{array}$}} & \multirow{2}{*}{\multicolumn{3}{|c|}{ Projected }} \\
\hline & & & & & & & & & & \\
\hline & 2002 & 2003 & 2004 & 2005 & 2006 & 2007 & 2008 & 2009 & 2010 & 2011 \\
\hline United States & 14,069 & 14,339 & 14,618 & 14,909 & 15,081 & 15,087 & 14,980 & 14,842 & 14,668 & 14,530 \\
\hline Northeast & 2,487 & 2,541 & 2,582 & 2,617 & 2,684 & 2,618 & 2,576 & 2,525 & 2,474 & 2,428 \\
\hline Connecticut & 164 & 169 & 173 & 175 & 177 & 177 & 175 & 172 & 169 & 167 \\
\hline Maine & 63 & 63 & 63 & 62 & 62 & 66 & 64 & 63 & 61 & 60 \\
\hline Massachusetts & 282 & 288 & 293 & 297 & 298 & 296 & 292 & 289 & 284 & 281 \\
\hline New Hampshire & 64 & 65 & 67 & 67 & 67 & 66 & 65 & 64 & 62 & 61 \\
\hline New Jersey & 389 & 402 & 417 & 425 & 425 & 428 & 425 & 421 & 419 & 417 \\
\hline New York & 872 & 887 & 894 & 907 & 922 & 909 & 898 & 877 & 855 & 834 \\
\hline Pennsylvania & 575 & 586 & 593 & 603 & 651 & 597 & 581 & 566 & 553 & 538 \\
\hline Rhode Island & 47 & 48 & 49 & 50 & 50 & 48 & 47 & 46 & 44 & 43 \\
\hline Vermont & 32 & 32 & 32 & 32 & 32 & 31 & 30 & 29 & 28 & 27 \\
\hline Midwest & 3,284 & 3,307 & 3,337 & 3,394 & 3,415 & 3,411 & 3,370 & 3,317 & 3,261 & 3,208 \\
\hline Illinois & 597 & 608 & 614 & 631 & 641 & 640 & 641 & 638 & 636 & 627 \\
\hline Indiana & 290 & 294 & 301 & 311 & 316 & 317 & 316 & 314 & 313 & 312 \\
\hline lowa & 156 & 154 & 154 & 157 & 157 & 156 & 152 & 149 & 146 & 145 \\
\hline Kansas & 149 & 148 & 148 & 147 & 143 & 142 & 140 & 139 & 138 & 137 \\
\hline Michigan & 531 & 528 & 540 & 551 & 552 & 556 & 541 & 524 & 507 & 490 \\
\hline Minnesota & 279 & 279 & 280 & 281 & 282 & 279 & 276 & 271 & 266 & 263 \\
\hline Missouri & 272 & 274 & 277 & 283 & 286 & 285 & 282 & 278 & 272 & 267 \\
\hline Nebraska & 90 & 90 & 91 & 92 & 92 & 91 & 90 & 88 & 87 & 86 \\
\hline North Dakota & 35 & 34 & 33 & 33 & 32 & 32 & 31 & 30 & 29 & 29 \\
\hline Ohio & 554 & 567 & 573 & 578 & 584 & 586 & 578 & 567 & 555 & 544 \\
\hline South Dakota & 41 & 40 & 39 & 38 & 38 & 38 & 39 & 40 & 40 & 40 \\
\hline Wisconsin & 290 & 290 & 287 & 291 & 292 & 289 & 284 & 278 & 272 & 268 \\
\hline South & 4,898 & 4,998 & 5,112 & 5,221 & 5,304 & 5,338 & 5,324 & 5,301 & 5,260 & 5,241 \\
\hline Alabama & 206 & 206 & 208 & 212 & 215 & 218 & 218 & 216 & 213 & 211 \\
\hline Arkansas & 132 & 133 & 135 & 138 & 140 & 139 & 137 & 136 & 135 & 135 \\
\hline Delaware & 34 & 35 & 35 & 36 & 37 & 38 & 39 & 38 & 38 & 38 \\
\hline District of Columbia & 17 & 19 & 20 & 21 & 20 & 23 & 18 & 18 & 17 & 17 \\
\hline Florida & 731 & 755 & 782 & 802 & 805 & 811 & 782 & 766 & 751 & 743 \\
\hline Georgia & 407 & 419 & 435 & 453 & 463 & 471 & 470 & 468 & 466 & 463 \\
\hline Kentucky & 184 & 185 & 189 & 192 & 196 & 197 & 198 & 196 & 194 & 192 \\
\hline Louisiana & 194 & 191 & 191 & 172 & 184 & 181 & 181 & 180 & 180 & 177 \\
\hline Maryland & 256 & 263 & 268 & 271 & 273 & 269 & 267 & 264 & 260 & 255 \\
\hline Mississippi & 132 & 133 & 134 & 137 & 139 & 141 & 140 & 139 & 137 & 134 \\
\hline North Carolina & 372 & 386 & 400 & 413 & 417 & 417 & 430 & 434 & 436 & 436 \\
\hline Oklahoma & 176 & 176 & 177 & 178 & 179 & 179 & 177 & 176 & 174 & 173 \\
\hline South Carolina & 194 & 198 & 199 & 204 & 207 & 208 & 211 & 209 & 206 & 204 \\
\hline Tennessee & 254 & 261 & 270 & 277 & 286 & 283 & 287 & 284 & 281 & 281 \\
\hline Texas & 1,180 & 1,199 & 1,221 & 1,257 & 1,280 & 1,300 & 1,306 & 1,315 & 1,319 & 1,335 \\
\hline Virginia & 346 & 355 & 365 & 372 & 379 & 380 & 381 & 377 & 373 & 367 \\
\hline West Virginia & 82 & 82 & 83 & 84 & 84 & 84 & 83 & 83 & 81 & 80 \\
\hline West & 3,399 & 3,494 & 3,587 & 3,677 & 3,678 & 3,720 & 3,710 & 3,698 & 3,673 & 3,654 \\
\hline Alaska & 40 & 40 & 41 & 42 & 42 & 42 & 41 & 41 & 39 & 39 \\
\hline Arizona & 277 & 308 & 321 & 355 & 309 & 316 & 316 & 312 & 312 & 314 \\
\hline California & 1,828 & 1,874 & 1,934 & 1,972 & 1,997 & 2,015 & 2,016 & 2,018 & 2,002 & 1,982 \\
\hline Colorado & 217 & 221 & 225 & 230 & 235 & 236 & 238 & 240 & 241 & 242 \\
\hline Hawaii & 53 & 54 & 54 & 55 & 55 & 54 & 54 & 52 & 52 & 51 \\
\hline Idaho & 75 & 77 & 78 & 79 & 80 & 81 & 82 & 82 & 82 & 82 \\
\hline Montana & 49 & 48 & 48 & 48 & 47 & 46 & 45 & 44 & 43 & 42 \\
\hline Nevada & 99 & 105 & 111 & 116 & 122 & 122 & 125 & 127 & 129 & 131 \\
\hline New Mexico & 96 & 97 & 98 & 97 & 98 & 99 & 99 & 97 & 96 & 95 \\
\hline Oregon & 172 & 173 & 176 & 173 & 182 & 182 & 180 & 177 & 176 & 176 \\
\hline Utah & 147 & 147 & 148 & 151 & 152 & 166 & 155 & 152 & 149 & 149 \\
\hline Washington & 318 & 322 & 325 & 333 & 332 & 333 & 332 & 330 & 327 & 326 \\
\hline Wyoming & 28 & 28 & 27 & 27 & 27 & 27 & 27 & 26 & 26 & 26 \\
\hline
\end{tabular}

See notes at end of table. 
Table 10. Actual and projected numbers for enrollment in grades 9-12 in public schools, by region and state: Fall 2002 through fall 2020-Continued

[In thousands]

\begin{tabular}{|c|c|c|c|c|c|c|c|c|c|}
\hline \multirow[b]{2}{*}{ Region and state } & \multicolumn{9}{|c|}{ Projected-Continued } \\
\hline & 2012 & 2013 & 2014 & 2015 & 2016 & 2017 & 2018 & 2019 & 2020 \\
\hline United States & 14,512 & 14,545 & 14,689 & 14,830 & 14,877 & 14,939 & 15,000 & 15,083 & 15,222 \\
\hline Northeast & 2,396 & 2,371 & 2,363 & 2,358 & 2,343 & 2,335 & 2,329 & 2,326 & 2,331 \\
\hline Connecticut & 166 & 165 & 163 & 162 & 160 & 159 & 158 & 158 & 158 \\
\hline Maine & 59 & 58 & 58 & 57 & 57 & 57 & 57 & 58 & 58 \\
\hline Massachusetts & 279 & 277 & 276 & 276 & 274 & 273 & 272 & 270 & 269 \\
\hline New Hampshire & 60 & 59 & 58 & 58 & 57 & 56 & 55 & 55 & 55 \\
\hline New Jersey & 416 & 416 & 417 & 418 & 418 & 418 & 419 & 420 & 421 \\
\hline New York & 820 & 807 & 803 & 800 & 794 & 792 & 789 & 788 & 788 \\
\hline Pennsylvania & 528 & 523 & 522 & 522 & 518 & 515 & 513 & 512 & 515 \\
\hline Rhode Island & 42 & 40 & 39 & 39 & 39 & 39 & 40 & 40 & 40 \\
\hline Vermont & 26 & 26 & 26 & 25 & 25 & 25 & 26 & 26 & 27 \\
\hline Midwest & 3,189 & 3,185 & 3,206 & 3,232 & 3,226 & 3,222 & 3,215 & 3,210 & 3,224 \\
\hline Illinois & 622 & 622 & 627 & 632 & 630 & 629 & 627 & 625 & 628 \\
\hline Indiana & 311 & 310 & 312 & 316 & 316 & 316 & 314 & 312 & 312 \\
\hline lowa & 145 & 146 & 148 & 149 & 150 & 151 & 152 & 153 & 154 \\
\hline Kansas & 138 & 139 & 142 & 144 & 146 & 147 & 147 & 148 & 149 \\
\hline Michigan & 484 & 479 & 477 & 475 & 467 & 458 & 451 & 447 & 448 \\
\hline Minnesota & 262 & 263 & 266 & 271 & 273 & 277 & 280 & 282 & 287 \\
\hline Missouri & 268 & 269 & 271 & 273 & 273 & 273 & 273 & 273 & 274 \\
\hline Nebraska & 86 & 87 & 88 & 90 & 92 & 94 & 95 & 96 & 97 \\
\hline North Dakota & 28 & 28 & 28 & 27 & 27 & 27 & 28 & 28 & 29 \\
\hline Ohio & 539 & 538 & 541 & 544 & 541 & 538 & 535 & 531 & 531 \\
\hline South Dakota & 40 & 40 & 40 & 40 & 41 & 42 & 42 & 43 & 44 \\
\hline Wisconsin & 266 & 266 & 268 & 270 & 271 & 271 & 271 & 271 & 274 \\
\hline South & 5,278 & 5,332 & 5,434 & 5,523 & 5,570 & 5,609 & 5,640 & 5,676 & 5,726 \\
\hline Alabama & 211 & 213 & 217 & 219 & 218 & 216 & 214 & 212 & 211 \\
\hline Arkansas & 136 & 138 & 140 & 142 & 144 & 144 & 144 & 144 & 144 \\
\hline Delaware & 38 & 38 & 39 & 40 & 41 & 41 & 42 & 42 & 43 \\
\hline District of Columbia & 16 & 15 & 15 & 14 & 14 & 14 & 15 & 16 & 17 \\
\hline Florida & 742 & 740 & 741 & 741 & 736 & 729 & 726 & 728 & 736 \\
\hline Georgia & 467 & 474 & 483 & 493 & 497 & 499 & 500 & 501 & 504 \\
\hline Kentucky & 193 & 195 & 196 & 198 & 199 & 200 & 200 & 200 & 200 \\
\hline Louisiana & 177 & 178 & 180 & 184 & 183 & 183 & 181 & 181 & 180 \\
\hline Maryland & 251 & 247 & 247 & 246 & 248 & 251 & 253 & 257 & 259 \\
\hline Mississippi & 133 & 133 & 135 & 137 & 137 & 137 & 136 & 135 & 135 \\
\hline North Carolina & 439 & 449 & 463 & 476 & 481 & 484 & 485 & 486 & 489 \\
\hline Oklahoma & 175 & 178 & 181 & 184 & 186 & 187 & 188 & 188 & 188 \\
\hline South Carolina & 205 & 208 & 213 & 218 & 219 & 219 & 218 & 217 & 218 \\
\hline Tennessee & 284 & 288 & 293 & 298 & 299 & 299 & 298 & 298 & 299 \\
\hline Texas & 1,363 & 1,392 & 1,435 & 1,472 & 1,505 & 1,540 & 1,569 & 1,599 & 1,629 \\
\hline Virginia & 367 & 368 & 372 & 378 & 382 & 385 & 387 & 389 & 392 \\
\hline West Virginia & 80 & 80 & 81 & 82 & 82 & 83 & 83 & 83 & 83 \\
\hline West & 3,650 & 3,657 & 3,686 & 3,718 & 3,738 & 3,773 & 3,816 & 3,872 & 3,941 \\
\hline Alaska & 38 & 39 & 39 & 40 & 41 & 41 & 42 & 43 & 45 \\
\hline Arizona & 319 & 327 & 335 & 344 & 351 & 356 & 363 & 369 & 378 \\
\hline California & 1,963 & 1,944 & 1,941 & 1,940 & 1,939 & 1,954 & 1,971 & 1,999 & 2,034 \\
\hline Colorado & 246 & 251 & 258 & 265 & 271 & 277 & 282 & 285 & 288 \\
\hline Hawaii & 50 & 50 & 50 & 50 & 50 & 51 & 52 & 53 & 54 \\
\hline Idaho & 83 & 85 & 86 & 88 & 90 & 91 & 93 & 94 & 96 \\
\hline Montana & 42 & 42 & 42 & 42 & 43 & 43 & 43 & 44 & 44 \\
\hline Nevada & 133 & 137 & 141 & 146 & 148 & 149 & 150 & 152 & 155 \\
\hline New Mexico & 94 & 95 & 97 & 98 & 100 & 101 & 103 & 105 & 106 \\
\hline Oregon & 177 & 179 & 180 & 182 & 182 & 182 & 183 & 185 & 188 \\
\hline Utah & 150 & 151 & 152 & 152 & 150 & 152 & 154 & 157 & 162 \\
\hline Washington & 329 & 332 & 337 & 341 & 344 & 346 & 349 & 353 & 360 \\
\hline Wyoming & 26 & 27 & 28 & 29 & 29 & 30 & 31 & 32 & 32 \\
\hline
\end{tabular}

NOTE: Some data have been revised from previously published figures. Detail may not sum to totals because of rounding. Mean absolute percentage errors of grades 9-12 enrollment in public schools by state and region can be found in table A-9, appendix A.

SOURCE: U.S. Department of Education, National Center for Education Statistics, Common Core of Data (CCD), "State Nonfiscal Survey of Public Elementary/Secondary Education," 2002-03 through 2008-09; and State Public Elementary and Secondary Enrollment Model, 1980-2008. (This table was prepared January 2011.) 
Table 11. Actual and projected percentage changes in grades 9-12 enrollment in public schools, by region and state: Selected years, fall 2002 through fall 2020

\begin{tabular}{|c|c|c|c|c|}
\hline \multirow[b]{2}{*}{ Region and state } & \multirow[b]{2}{*}{ Actual 2002-2008 } & \multicolumn{3}{|c|}{ Projected } \\
\hline & & 2008-2014 & 2014-2020 & 2008-2020 \\
\hline United States & 6.5 & -1.9 & 3.6 & 1.6 \\
\hline Northeast & 3.6 & -8.3 & -1.4 & -9.5 \\
\hline Connecticut & 6.7 & -6.6 & -3.5 & -9.9 \\
\hline Maine & 1.7 & -9.2 & 1.0 & -8.4 \\
\hline Massachusetts & 3.7 & -5.4 & -2.8 & -8.1 \\
\hline New Hampshire & 1.4 & -10.1 & -5.5 & -15.1 \\
\hline New Jersey & 9.2 & -1.7 & 0.9 & -0.9 \\
\hline New York & 2.9 & -10.5 & -1.9 & -12.2 \\
\hline Pennsylvania & 1.0 & -10.1 & -1.3 & -11.3 \\
\hline Rhode Island & 1.5 & -18.1 & 3.4 & -15.4 \\
\hline Vermont & -6.0 & -14.1 & 2.9 & -11.7 \\
\hline Midwest & 2.6 & -4.8 & 0.6 & -4.3 \\
\hline Illinois & 7.4 & -2.2 & 0.2 & -2.0 \\
\hline Indiana & 9.1 & -1.5 & 0.2 & -1.2 \\
\hline lowa & -2.8 & -2.9 & 4.0 & 1.0 \\
\hline Kansas & -6.2 & 1.1 & 5.0 & 6.2 \\
\hline Michigan & 1.9 & -11.8 & -6.2 & -17.3 \\
\hline Minnesota & -1.2 & -3.5 & 8.0 & 4.2 \\
\hline Missouri & 3.9 & -3.9 & 0.8 & -3.1 \\
\hline Nebraska & -0.7 & -1.7 & 10.0 & 8.0 \\
\hline North Dakota & -12.4 & -10.3 & 3.8 & -6.9 \\
\hline Ohio & 4.2 & -6.4 & -1.9 & -8.1 \\
\hline South Dakota & -3.7 & 2.4 & 8.6 & 11.3 \\
\hline Wisconsin & -1.8 & -5.8 & 2.2 & -3.8 \\
\hline South & 8.7 & 2.1 & 5.4 & 7.6 \\
\hline Alabama & 5.5 & -0.4 & -2.6 & -3.0 \\
\hline Arkansas & 3.9 & 2.1 & 2.4 & 4.5 \\
\hline Delaware & 13.2 & 1.7 & 8.9 & 10.8 \\
\hline District of Columbia & 3.1 & -17.3 & 15.9 & -4.2 \\
\hline Florida & 7.0 & -5.2 & -0.7 & -5.8 \\
\hline Georgia & 15.4 & 2.8 & 4.3 & 7.2 \\
\hline Kentucky & 7.5 & -0.7 & 1.6 & 0.9 \\
\hline Louisiana & -6.7 & -0.1 & -0.1 & -0.2 \\
\hline Maryland & 4.3 & -7.7 & 5.1 & -3.0 \\
\hline Mississippi & 5.9 & -3.4 & -0.3 & -3.8 \\
\hline North Carolina & 15.5 & 7.7 & 5.7 & 13.9 \\
\hline Oklahoma & 0.9 & 2.3 & 3.8 & 6.2 \\
\hline South Carolina & 8.5 & 1.2 & 2.2 & 3.5 \\
\hline Tennessee & 13.0 & 2.0 & 1.8 & 3.9 \\
\hline Texas & 10.6 & 9.9 & 13.5 & 24.7 \\
\hline Virginia & 10.1 & -2.2 & 5.3 & 3.0 \\
\hline West Virginia & 1.0 & -2.6 & 1.8 & -0.9 \\
\hline West & 9.1 & -0.7 & 6.9 & 6.2 \\
\hline Alaska & 3.5 & -5.2 & 13.4 & 7.5 \\
\hline Arizona & 13.9 & 6.1 & 12.6 & 19.5 \\
\hline California & 10.3 & -3.8 & 4.8 & 0.9 \\
\hline Colorado & 9.5 & 8.1 & 11.9 & 21.0 \\
\hline Hawaii & 1.1 & -6.8 & 7.4 & 0.2 \\
\hline Idaho & 8.3 & 5.4 & 11.1 & 17.1 \\
\hline Montana & -7.8 & -6.7 & 5.2 & -1.9 \\
\hline Nevada & 26.9 & 12.8 & 9.6 & 23.6 \\
\hline New Mexico & 3.2 & -2.1 & 9.9 & 7.6 \\
\hline Oregon & 4.6 & 0.1 & 4.5 & 4.6 \\
\hline Utah & 5.9 & -1.8 & 6.3 & 4.3 \\
\hline Washington & 4.6 & 1.4 & 6.8 & 8.3 \\
\hline Wyoming & -5.9 & 4.7 & 16.0 & 21.5 \\
\hline
\end{tabular}

NOTE: Calculations are based on unrounded numbers. Mean absolute percentage errors of grades 9-12 enrollment in public schools by state and region can be found in table $A-9$, appendix $A$.

SOURCE: U.S. Department of Education, National Center for Education Statistics, Common Core of Data (CCD), "State Nonfiscal Survey of Public Elementary/Secondary Education," selected years, 2002-03 through 2008-09; and State Public Elementary and Secondary Enrollment Model, 1980-2008. (This table was prepared January 2011.) 
Table 12. Actual and projected numbers for high school graduates, by control of school: School years 1995-96 through 2020-21

\begin{tabular}{|c|c|c|c|}
\hline School year & Total & Public & Private \\
\hline \multicolumn{4}{|l|}{ Actual } \\
\hline $1995-96^{1}$ & $2,518,064$ & $2,273,109$ & 244,955 \\
\hline 1996-97 & $2,611,988$ & $2,358,403$ & 253,585 \\
\hline $1997-98^{1}$ & $2,704,133$ & $2,439,050$ & 265,083 \\
\hline 1998-99 & $2,758,655$ & $2,485,630$ & 273,025 \\
\hline 1999-20001 & $2,832,656$ & $2,553,844$ & 278,812 \\
\hline 2000-01 & $2,847,973$ & $2,569,200$ & 278,773 \\
\hline $2001-02^{1}$ & $2,906,287$ & $2,621,534$ & 284,753 \\
\hline 2002-03 & $3,015,702$ & $2,719,947$ & 295,755 \\
\hline $2003-04^{1}$ & $3,054,247$ & $2,753,438$ & 300,809 \\
\hline 2004-05 & $3,106,499$ & $2,799,250$ & 307,249 \\
\hline $2005-06^{1}$ & $3,119,294$ & $2,815,544$ & 303,750 \\
\hline $2006-07$ & $3,198,956$ & $2,892,351$ & 306,605 \\
\hline $2007-08^{1}$ & $3,311,771$ & $2,999,508$ & 312,263 \\
\hline \multicolumn{4}{|l|}{ Projected } \\
\hline 2008-09 & $3,330,340$ & $3,021,530$ & 308,810 \\
\hline 2009-10 & $3,321,380$ & $3,013,400$ & 307,980 \\
\hline 2010-11 & $3,282,200$ & $2,981,520$ & 300,680 \\
\hline 2011-12 & $3,220,050$ & $2,926,440$ & 293,610 \\
\hline $2012-13$ & $3,197,300$ & $2,912,480$ & 284,820 \\
\hline 2013-14 & $3,153,500$ & $2,874,500$ & 279,000 \\
\hline 2014-15 & $3,132,400$ & $2,867,400$ & 265,000 \\
\hline 2015-16 & $3,164,790$ & $2,904,410$ & 260,380 \\
\hline $2016-17$ & $3,182,750$ & $2,930,530$ & 252,220 \\
\hline 2017-18 & $3,231,350$ & $2,983,130$ & 248,220 \\
\hline 2018-19 & $3,224,820$ & $2,983,780$ & 241,040 \\
\hline 2019-20 & $3,182,520$ & $2,950,880$ & 231,640 \\
\hline 2020-21 & $3,209,510$ & $2,981,170$ & 228,340 \\
\hline
\end{tabular}

${ }^{1}$ Since the biennial Private School Universe Survey (PSS) is collected in the fall of odd numbered years, private school numbers for alternate years are estimated based on data from the PSS.

NOTE: Some data have been revised from previously published figures. Detail may not sum to totals because of rounding. Mean absolute percentage errors of selected education statistics can be found in table A-2, appendix A.

SOURCE: U.S. Department of Education, National Center for Education Statistics, Common Core of Data (CCD), "State Nonfiscal Survey of Public Elementary/Secondary Education," 1996-97 through 2008-09; Private School Universe Survey (PSS), selected years, 1995-96 through 2007-08; and National Elementary and Secondary High School Graduates Model, 1972-73 through 2009-10. (This table was prepared January 2011.) 
Table 13. Actual and projected numbers for public high school graduates, by race/ethnicity: School years 1995-96 through 2020-21

\begin{tabular}{|c|c|c|c|c|c|c|}
\hline \multirow[b]{2}{*}{ School year } & \multirow[b]{2}{*}{ Total } & \multicolumn{5}{|c|}{ Race/ethnicity } \\
\hline & & White & Black & Hispanic & $\begin{array}{r}\text { Asian/Pacific } \\
\text { Islander }\end{array}$ & $\begin{array}{r}\text { American Indian/ } \\
\text { Alaska Native }\end{array}$ \\
\hline \multicolumn{7}{|l|}{ Actual } \\
\hline 1995-96 & $2,273,109$ & $1,639,420$ & 294,253 & 218,785 & 99,189 & 21,463 \\
\hline 1996-97 & $2,358,403$ & $1,687,681$ & 309,626 & 234,043 & 104,764 & 22,287 \\
\hline $1997-98$ & $2,439,050$ & $1,733,478$ & 317,846 & 252,023 & 112,089 & 23,614 \\
\hline 1998-99 & $2,485,630$ & $1,754,619$ & 322,532 & 268,539 & 115,930 & 24,012 \\
\hline 1999-2000 & $2,553,844$ & $1,787,322$ & 334,206 & 283,738 & 123,231 & 25,347 \\
\hline 2000-01 & $2,569,200$ & $1,782,292$ & 336,375 & 297,696 & 126,847 & 25,988 \\
\hline 2001-02 & $2,621,534$ & $1,801,173$ & 345,854 & 314,989 & 132,347 & 27,169 \\
\hline 2002-03 & $2,719,947$ & $1,857,957$ & 359,051 & 339,555 & 135,614 & 27,773 \\
\hline 2003-04 & $2,753,438$ & $1,851,136$ & 373,307 & 362,467 & 137,913 & 28,615 \\
\hline 2004-05 & $2,799,250$ & $1,856,758$ & 385,180 & 382,964 & 143,751 & 30,595 \\
\hline 2005-06 & $2,815,544$ & $1,854,775$ & 392,180 & 388,718 & 150,567 & 29,303 \\
\hline $2006-07$ & $2,892,351$ & $1,883,601$ & 412,640 & 410,443 & 154,871 & 30,795 \\
\hline 2007-08 & $2,999,508$ & $1,916,642$ & 435,920 & 453,383 & 161,164 & 32,401 \\
\hline \multicolumn{7}{|l|}{ Projected } \\
\hline 2008-09 & $3,021,530$ & $1,893,700$ & 450,650 & 480,280 & 164,290 & 32,610 \\
\hline $2009-10$ & $3,013,400$ & $1,860,090$ & 457,100 & 496,670 & 166,920 & 32,630 \\
\hline $2010-11$ & $2,981,520$ & $1,814,440$ & 460,110 & 504,600 & 170,270 & 31,550 \\
\hline 2011-12 & $2,926,440$ & $1,769,560$ & 448,240 & 504,270 & 173,650 & 30,160 \\
\hline $2012-13$ & $2,912,480$ & $1,748,920$ & 440,710 & 514,210 & 178,360 & 29,730 \\
\hline $2013-14$ & $2,874,500$ & $1,721,130$ & 421,800 & 515,800 & 185,000 & 29,810 \\
\hline 2014-15 & $2,867,400$ & $1,703,760$ & 422,650 & 521,950 & 189,720 & 29,740 \\
\hline $2015-16$ & $2,904,410$ & $1,713,770$ & 432,320 & 532,280 & 192,750 & 31,050 \\
\hline $2016-17$ & $2,930,530$ & $1,722,770$ & 436,950 & 538,600 & 198,250 & 31,660 \\
\hline 2017-18 & $2,983,130$ & $1,733,340$ & 443,330 & 550,950 & 216,160 & 31,740 \\
\hline 2018-19 & $2,983,780$ & $1,726,110$ & 442,460 & 557,020 & 220,000 & 32,370 \\
\hline 2019-20 & $2,950,880$ & $1,695,060$ & 436,690 & 554,700 & 227,000 & 31,960 \\
\hline $2020-21$ & $2,981,170$ & $1,707,400$ & 425,940 & 575,060 & 234,550 & 32,690 \\
\hline
\end{tabular}

NOTE: Some data have been revised from previously published figures. Detail may not sum to totals because of rounding. The historical racial/ethnic time-series were constructed using racial/ethnic high school graduate data at the state level. In some instances, high school graduate data by race/ ethnicity had to be imputed. Further, in some instances, the racial/ethnic data had to be adjusted in order for them to sum to the state total for high school graduates. Race categories exclude persons of Hispanic ethnicity. For additional information see the High School Graduates section A.2 in appendix A. Mean absolute percentage errors of selected education statistics can be found in table A-2, appendix A.

SOURCE: U.S. Department of Education, National Center for Education Statistics, Common Core of Data (CCD), "State Nonfiscal Survey of Public Elementary/Secondary Education," 1996-97 through 2008-09; and National Public Elementary and Secondary High School Graduates Model by Race/Ethnicity Model, 1972-73 through 2007-08. (This table was prepared January 2011.) 
This page intentionally left blank. 
Table 14. Actual and projected numbers for public high school graduates, by region and state: School years $2002-03$ through 2020-21

\begin{tabular}{|c|c|c|c|c|c|c|c|c|c|c|}
\hline \multirow[b]{2}{*}{ Region and state } & \multicolumn{6}{|c|}{ Actual } & \multicolumn{4}{|c|}{ Projected } \\
\hline & 2002-03 & 2003-04 & 2004-05 & 2005-06 & 2006-07 & 2007-08 & 2008-09 & $2009-10$ & 2010-11 & 2011-12 \\
\hline United States & $2,719,947$ & $2,753,438$ & $2,799,250$ & $2,815,544$ & $2,892,351$ & $2,999,508$ & $3,021,530$ & $3,013,400$ & $2,981,520$ & $2,926,4$ \\
\hline Northeast & 477,241 & 485,670 & 503,528 & 521,015 & 536,697 & 552,289 & 545,240 & 540,220 & 528,450 & 518,650 \\
\hline Connecticut & 33,667 & 34,573 & 35,515 & 36,222 & 37,541 & 38,419 & 38,340 & 37,750 & 37,130 & 36,220 \\
\hline Maine & 12,947 & 13,278 & 13,077 & 12,950 & 13,151 & 14,350 & 13,570 & 13,970 & 13,280 & 13,200 \\
\hline Massachusetts & 55,987 & 58,326 & 59,665 & 61,272 & 63,903 & 65,197 & 64,040 & 63,650 & 61,470 & 61,270 \\
\hline New Hampshire & 13,210 & 13,309 & 13,775 & 13,988 & 14,452 & 14,982 & 14,440 & 14,480 & 13,890 & 13,680 \\
\hline New Jersey & 81,391 & 83,826 & 86,502 & 90,049 & 93,013 & 94,994 & 95,510 & 94,660 & 94,480 & 3,790 \\
\hline New York & 143,818 & 142,526 & 153,203 & 161,817 & 168,333 & 176,310 & 176,380 & 177,260 & 172,390 & 167,610 \\
\hline Pennsylvar & 119,933 & 123,474 & 124,758 & 127,830 & 128,603 & 130,298 & 125,850 & 121,890 & 119,940 & 117,300 \\
\hline Rhod & 9,318 & 9,258 & 9,881 & 10,108 & 10,384 & 10 & 40 & 9,480 & & 9,290 \\
\hline Vermont & 6,970 & 7,100 & 7,152 & 6,779 & 7,317 & 7,392 & 6,980 & 7,070 & 6,540 & ,290 \\
\hline Midwest & 673,248 & 680,178 & 676,786 & 684,049 & 702,987 & 721,220 & 724,990 & 715,570 & & 690,710 \\
\hline Illinois & 117,507 & 124,763 & 123,615 & 126,817 & & & 137,860 & & & 138,140 \\
\hline Indiana & 7 & 6,008 & 14 & 57,920 & 87 & & & & & 50 \\
\hline lowa & 34,860 & 34,339 & 33,547 & 33,693 & & & & & & \\
\hline & 3 & 55 & 3 & & & & & & & \\
\hline & 100,301 & 98,823 & 101,582 & 102 & 111 & 115 & 116 & 112 & & \\
\hline & & & & & & & & & & \\
\hline & & & & 7 & & & & & & \\
\hline & 2 & 99 & $19, \varsigma$ & 1 & 73 & & & & & \\
\hline & & & & & & & & & & 320 \\
\hline & 2 & 19,029 & 40 & 6 & 8 & 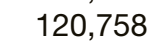 & 0 & 30 & 40 & 560 \\
\hline Sc & 8,999 & 9,001 & 8,585 & 8,589 & 8,346 & 8,5 & Do & 8,640 & & \\
\hline Wis & & & & & & & & & & 960 \\
\hline South & & & & 27 & & 0 & 046,660 & 051,540 & 045,530 & 023,570 \\
\hline Alabs & 1 & 36,464 & & 37 & 38,912 & & 41,310 & 40,630 & 41,650 & 41,010 \\
\hline & & & & & & & & & & 870 \\
\hline & & & & & & & & & & \\
\hline Dis & 25 & 31 & 81 & 50 & 44 & & 3,260 & & & 890 \\
\hline & & & & & 142,284 & & & & & \\
\hline & & & & & & & & & & \\
\hline & & & & & & & & & & \\
\hline & & & & & & & & & & \\
\hline & & & & & & & & & & \\
\hline & 0 & 35 & 2 & 18 & 36 & & & & & 25,470 \\
\hline & & & & & & & & & & \\
\hline & & & & & & & & & & \\
\hline So & & & & & & & & & & 37,350 \\
\hline & & & & & & & & & & \\
\hline & & & & & & & & & & 25 \\
\hline & & & & & & & & & & 76,920 \\
\hline & & & & & & & & & & \\
\hline West & & & & & & & & & 300 & 693,520 \\
\hline Alas & & & & & 7,666 & & & & 7,780 & 7,590 \\
\hline & & & & & & & & & & \\
\hline & & & & & & & & 38 & 40 & 37 \\
\hline Col & & 777 & 32 & 24 & 28 & & & 740 & 400 & 47,740 \\
\hline & & & & & & & & & & \\
\hline & & & & & & & & & & \\
\hline & 7 & 0 & & & & & 70 & & 9,430 & 9,260 \\
\hline & & & & & & & & & & \\
\hline & & & & & & & & & & \\
\hline & & & 3 & & & & 580 & 34,860 & 33,720 & 33,230 \\
\hline & & & & & 28,276 & & & & & 26,460 \\
\hline & & & & & & & & & & 62,020 \\
\hline Wyoming & 5,845 & 5,833 & 5,616 & 5,527 & 5,441 & 5,494 & 5,430 & 5,390 & 5,200 & 5,140 \\
\hline
\end{tabular}

See notes at end of table. 
Table 14. Actual and projected numbers for public high school graduates, by region and state: School years $2002-03$ through 2020-21-Continued

\begin{tabular}{|c|c|c|c|c|c|c|c|c|c|}
\hline \multirow[b]{2}{*}{ Region and state } & \multicolumn{9}{|c|}{ Projected-Continued } \\
\hline & 2012-13 & 2013-14 & 2014-15 & 2015-16 & $2016-17$ & 2017-18 & 2018-19 & 2019-20 & 2020-21 \\
\hline United States & $2,912,480$ & $2,874,500$ & $2,867,400$ & $2,904,410$ & $2,930,530$ & $2,983,130$ & $2,983,780$ & $2,950,880$ & $2,981,170$ \\
\hline Northeast & 510,180 & 498,260 & 490,680 & 491,270 & 488,800 & 490,950 & 486,690 & 478,550 & 481,850 \\
\hline Connecticut & 35,720 & 35,570 & 34,960 & 35,240 & 34,730 & 34,660 & 34,220 & 33,490 & 33,710 \\
\hline Maine & 12,790 & 12,490 & 12,420 & 12,410 & 12,090 & 12,170 & 12,190 & 12,040 & 12,120 \\
\hline Massachusetts & 60,960 & 59,600 & 58,950 & 59,820 & 58,940 & 59,120 & 58,710 & 58,010 & 58,080 \\
\hline New Hampshire & 13,330 & 13,040 & 12,890 & 12,840 & 12,550 & 12,510 & 12,330 & 12,170 & 11,860 \\
\hline New Jersey & 93,690 & 92,570 & 92,650 & 93,150 & 93,660 & 93,590 & 93,590 & 92,370 & 93,990 \\
\hline New York & 164,990 & 159,810 & 156,470 & 155,680 & 154,460 & 155,810 & 153,360 & 151,430 & 151,970 \\
\hline Pennsylvania & 113,860 & 110,860 & 108,220 & 108,020 & 109,290 & 109,910 & 108,570 & 105,500 & 106,440 \\
\hline Rhode Island & 8,750 & 8,480 & 8,240 & 8,300 & 7,300 & 7,560 & 8,110 & 7,950 & 8,000 \\
\hline Vermont & 6,100 & 5,850 & 5,870 & 5,800 & 5,780 & 5,610 & 5,610 & 5,590 & 5,690 \\
\hline Midwest & 680,690 & 669,420 & 665,870 & 673,470 & 677,250 & 687,250 & 688,110 & 671,510 & 674,490 \\
\hline Illinois & 135,760 & 133,480 & 131,960 & 133,230 & 135,210 & 137,280 & 136,900 & 132,280 & 134,050 \\
\hline Indiana & 61,640 & 61,700 & 60,740 & 60,830 & 61,360 & 62,450 & 64,230 & 61,100 & 61,140 \\
\hline lowa & 31,090 & 31,040 & 31,230 & 31,670 & 32,090 & 32,590 & 32,560 & 32,540 & 32,970 \\
\hline Kansas & 29,190 & 28,990 & 28,770 & 29,910 & 30,410 & 31,070 & 31,250 & 31,140 & 31,290 \\
\hline Michigan & 101,800 & 98,550 & 97,590 & 97,830 & 96,950 & 97,240 & 95,600 & 91,670 & 90,100 \\
\hline Minnesota & 55,790 & 55,100 & 55,610 & 56,040 & 56,770 & 58,150 & 59,370 & 58,130 & 59,900 \\
\hline Missouri & 58,720 & 57,980 & 58,150 & 59,580 & 59,260 & 60,380 & 60,130 & 59,590 & 59,380 \\
\hline Nebraska & 19,040 & 18,910 & 18,950 & 19,120 & 19,530 & 20,080 & 20,360 & 20,720 & 21,300 \\
\hline North Dakota & 6,240 & 6,130 & 6,140 & 6,080 & 5,980 & 5,770 & 5,970 & 5,880 & 6,090 \\
\hline Ohio & 112,870 & 109,890 & 109,450 & 111,190 & 111,250 & 112,410 & 112,310 & 109,370 & 108,920 \\
\hline South Dakota & 9,020 & 8,830 & 8,910 & 8,820 & 8,880 & 9,110 & 9,010 & 9,330 & 9,560 \\
\hline Wisconsin & 59,540 & 58,830 & 58,370 & 59,190 & 59,570 & 60,730 & 60,440 & 59,780 & 59,810 \\
\hline South & $1,029,680$ & $1,019,970$ & $1,028,350$ & $1,050,110$ & $1,068,770$ & $1,097,710$ & $1,102,230$ & $1,095,210$ & $1,103,230$ \\
\hline Alabama & 40,050 & 39,190 & 39,640 & 40,710 & 41,280 & 42,070 & 41,710 & 40,160 & 39,960 \\
\hline Arkansas & 27,450 & 27,530 & 28,030 & 28,420 & 29,010 & 29,190 & 29,720 & 29,550 & 29,330 \\
\hline Delaware & 7,730 & 7,760 & 7,810 & 7,980 & 8,330 & 8,450 & 8,550 & 8,430 & 8,770 \\
\hline District of Columbia & 2,650 & 2,470 & 2,400 & 2,370 & 2,210 & 2,290 & 2,170 & 1,980 & 2,180 \\
\hline Florida & 135,240 & 132,140 & 133,320 & 131,250 & 132,820 & 133,460 & 132,700 & 129,070 & 127,850 \\
\hline Georgia & 83,450 & 83,440 & 83,400 & 86,210 & 87,850 & 90,010 & 91,050 & 89,690 & 89,960 \\
\hline Kentucky & 39,770 & 39,290 & 39,280 & 40,550 & 40,830 & 40,900 & 40,870 & 41,190 & 41,340 \\
\hline Louisiana & 33,620 & 33,880 & 31,220 & 33,340 & 33,990 & 34,960 & 34,210 & 33,510 & 33,670 \\
\hline Maryland & 55,700 & 54,220 & 53,520 & 53,340 & 52,310 & 53,860 & 52,880 & 54,940 & 54,730 \\
\hline Mississippi & 25,430 & 24,390 & 24,080 & 24,400 & 25,000 & 26,080 & 25,440 & 24,560 & 25,150 \\
\hline North Carolina & 88,820 & 87,750 & 89,750 & 93,160 & 96,030 & 99,290 & 100,690 & 98,700 & 98,700 \\
\hline Oklahoma & 36,280 & 36,140 & 36,610 & 38,170 & 38,490 & 39,240 & 39,460 & 39,460 & 39,540 \\
\hline South Carolina & 36,960 & 36,190 & 36,560 & 37,810 & 38,670 & 39,960 & 40,070 & 39,090 & 39,030 \\
\hline Tennessee & 57,280 & 56,200 & 56,990 & 58,710 & 60,080 & 60,660 & 60,880 & 60,170 & 59,840 \\
\hline Texas & 266,010 & 267,820 & 274,970 & 280,660 & 288,320 & 301,180 & 305,530 & 308,140 & 316,560 \\
\hline Virginia & 76,100 & 74,960 & 74,190 & 76,110 & 76,700 & 78,650 & 79,120 & 79,050 & 79,270 \\
\hline West Virginia & 17,140 & 16,600 & 16,590 & 16,930 & 16,880 & 17,480 & 17,210 & 17,530 & 17,380 \\
\hline West & 691,930 & 686,850 & 682,500 & 689,570 & 695,710 & 707,220 & 706,750 & 705,610 & 721,610 \\
\hline Alaska & 7,380 & 7,240 & 7,190 & 7,270 & 7,580 & 7,770 & 7,850 & 7,770 & 7,950 \\
\hline Arizona & 59,250 & 59,760 & 60,940 & 62,400 & 64,760 & 66,430 & 67,690 & 67,760 & 68,930 \\
\hline California & 375,370 & 368,770 & 361,470 & 360,220 & 357,590 & 361,940 & 357,090 & 354,850 & 364,000 \\
\hline Colorado & 47,960 & 48,360 & 48,900 & 50,460 & 51,770 & 53,800 & 54,820 & 55,500 & 56,760 \\
\hline Hawaii & 10,310 & 10,720 & 10,120 & 10,280 & 10,300 & 10,590 & 10,010 & 10,460 & 10,790 \\
\hline Idaho & 16,940 & 17,460 & 17,060 & 17,590 & 18,340 & 18,450 & 19,010 & 19,080 & 19,150 \\
\hline Montana & 9,020 & 9,010 & 8,880 & 8,990 & 9,060 & 9,020 & 9,170 & 9,210 & 9,210 \\
\hline Nevada & 19,610 & 19,880 & 19,860 & 20,670 & 21,570 & 22,380 & 22,730 & 22,590 & 22,470 \\
\hline New Mexico & 17,570 & 17,150 & 17,490 & 17,620 & 18,080 & 18,290 & 18,680 & 18,680 & 19,040 \\
\hline Oregon & 33,600 & 33,630 & 33,580 & 34,350 & 34,780 & 34,820 & 34,800 & 34,570 & 34,780 \\
\hline Utah & 27,390 & 27,970 & 29,070 & 30,450 & 31,450 & 32,260 & 32,850 & 33,420 & 35,540 \\
\hline Washington & 62,550 & 61,780 & 62,680 & 63,780 & 64,850 & 65,730 & 66,180 & 65,650 & 66,650 \\
\hline Wyoming & 4,980 & 5,130 & 5,260 & 5,500 & 5,590 & 5,750 & 5,890 & 6,070 & 6,340 \\
\hline
\end{tabular}

NOTE: Some data have been revised from previously published figures. Detail may not sum to totals because of rounding. Mean absolute percentage errors of public high school graduates by state and region can be found in table A-10, appendix A.

SOURCE: U.S. Department of Education, National Center for Education Statistics, Common Core of Data (CCD), "State Nonfiscal Survey of Public Elementary/Secondary Education," 2003-04 through 2008-09; and State Public High School Graduates Model, 1980-81 through 2007-08. (This table was prepared January 2011.) 
Table 15. Actual and projected percentage changes in public high school graduates, by region and state: Selected school years, 2002-03 through 2020-21

\begin{tabular}{|c|c|c|c|c|}
\hline \multirow[b]{2}{*}{ Region and state } & \multirow{2}{*}{$\begin{array}{r}\text { Actual } 2002-03 \\
\text { to } 2007-08 \\
\end{array}$} & \multicolumn{3}{|c|}{ Projected } \\
\hline & & $2007-08$ to $2014-15$ & $2014-15$ to $2020-21$ & $2007-08$ to $2020-21$ \\
\hline United States & 10.3 & -4.4 & 4.0 & -0.6 \\
\hline Northeast & 15.7 & -11.2 & -1.8 & -12.8 \\
\hline Connecticut & 14.1 & -9.0 & -3.6 & -12.3 \\
\hline Maine & 10.8 & -13.4 & -2.4 & -15.5 \\
\hline Massachusetts & 16.5 & -9.6 & -1.5 & -10.9 \\
\hline New Hampshire & 13.4 & -14.0 & -8.0 & -20.8 \\
\hline New Jersey & 16.7 & -2.5 & 1.4 & -1.1 \\
\hline New York & 22.6 & -11.3 & -2.9 & -13.8 \\
\hline Pennsylvania & 8.6 & -16.9 & -1.6 & -18.3 \\
\hline Rhode Island & 11.0 & -20.4 & -2.9 & -22.7 \\
\hline Vermont & 6.1 & -20.6 & -3.1 & -23.0 \\
\hline Midwest & 7.1 & -7.7 & 1.3 & -6.5 \\
\hline Illinois & 15.0 & -2.4 & 1.6 & -0.8 \\
\hline Indiana & 6.9 & -1.9 & 0.7 & -1.2 \\
\hline lowa & -0.8 & -9.7 & 5.6 & -4.6 \\
\hline Kansas & 2.6 & -6.4 & 8.8 & 1.8 \\
\hline Michigan & 14.8 & -15.3 & -7.7 & -21.8 \\
\hline Minnesota & 1.6 & -7.9 & 7.7 & -0.8 \\
\hline Missouri & 8.4 & -5.8 & 2.1 & -3.8 \\
\hline Nebraska & -0.6 & -5.4 & 12.4 & 6.3 \\
\hline North Dakota & -14.3 & -12.3 & -0.8 & -13.0 \\
\hline Ohio & 4.3 & -9.4 & -0.5 & -9.8 \\
\hline South Dakota & -4.6 & 3.8 & 7.3 & 11.4 \\
\hline Wisconsin & 3.0 & -10.5 & 2.5 & -8.2 \\
\hline South & 10.9 & -0.3 & 7.3 & 6.9 \\
\hline Alabama & 12.5 & -4.1 & 0.8 & -3.4 \\
\hline Arkansas & 4.2 & -2.4 & 4.6 & 2.1 \\
\hline Delaware & 8.4 & 5.7 & 12.3 & 18.7 \\
\hline District of Columbia & 23.0 & -28.4 & -9.2 & -35.0 \\
\hline Florida & 16.9 & -10.6 & -4.1 & -14.2 \\
\hline Georgia & 24.8 & -0.1 & -4.1 & 7.7 \\
\hline Kentucky & 4.5 & -0.1 & 5.2 & 5.1 \\
\hline Louisiana & -8.5 & -9.2 & 7.8 & -2.1 \\
\hline Maryland & 14.1 & -9.6 & 2.3 & -7.5 \\
\hline Mississippi & 4.1 & -2.9 & 4.4 & 1.4 \\
\hline North Carolina & 19.5 & 7.7 & 10.0 & 18.5 \\
\hline Oklahoma & 2.6 & -2.7 & 8.0 & 5.1 \\
\hline South Carolina & 8.2 & 4.0 & 6.8 & 11.1 \\
\hline Tennessee & 30.3 & -0.9 & 5.0 & 4.1 \\
\hline Texas & 5.9 & 9.1 & 15.1 & 25.6 \\
\hline Virginia & 6.1 & -4.1 & 6.8 & 2.5 \\
\hline West Virginia & 1.2 & -5.1 & 4.8 & -0.6 \\
\hline West & 8.7 & -1.7 & 5.7 & 3.9 \\
\hline Alaska & 7.6 & -8.5 & 10.6 & 1.2 \\
\hline Arizona & 23.4 & -1.2 & 13.1 & 11.8 \\
\hline California & 9.8 & -3.5 & 0.7 & -2.8 \\
\hline Colorado & 8.7 & 6.1 & 16.1 & 23.2 \\
\hline Hawaii & 16.0 & -12.9 & 6.6 & -7.1 \\
\hline Idaho & 4.5 & 3.0 & 12.3 & 15.6 \\
\hline Montana & -2.4 & -14.6 & 3.7 & -11.4 \\
\hline Nevada & 4.7 & 15.8 & 13.1 & 31.0 \\
\hline New Mexico & 7.9 & -4.2 & 8.9 & 4.2 \\
\hline Oregon & 7.2 & -3.9 & 3.6 & -0.5 \\
\hline Utah & -4.6 & 3.2 & 22.3 & 26.2 \\
\hline Washington & 2.0 & 1.7 & 6.3 & 8.2 \\
\hline Wyoming & -6.0 & -4.3 & 20.5 & 15.4 \\
\hline
\end{tabular}

NOTE: Calculations are based on unrounded numbers. Mean absolute percentage errors of public high school graduates by state and region can be found in table A-10, appendix A.

SOURCE: U.S. Department of Education, National Center for Education Statistics, Common Core of Data (CCD), "State Nonfiscal Survey of Public Elementary/Secondary Education," 2003-04 and 2008-09; and State Public High School Graduates Model, 1980-81 through 2007-08. (This table was prepared January 2011.) 
Table 16. Actual and projected numbers for elementary and secondary teachers and elementary and secondary new teacher hires, by control of school: Fall 1995 through fall 2020

[In thousands]

\begin{tabular}{|c|c|c|c|c|c|c|}
\hline \multirow[b]{3}{*}{ Year } & \multicolumn{3}{|c|}{ Number of teachers } & \multicolumn{3}{|c|}{ Number of new teacher hires } \\
\hline & \multirow[b]{2}{*}{ Total } & \multicolumn{2}{|c|}{ Control } & \multirow[b]{2}{*}{ Total } & \multicolumn{2}{|c|}{ Control } \\
\hline & & Public & Private & & Public & Private \\
\hline \multicolumn{7}{|l|}{ Actual } \\
\hline 1995 & 2,974 & 2,598 & 376 & - & - & - \\
\hline 19961 & 3,051 & 2,667 & 384 & - & - & - \\
\hline 1997 & 3,138 & 2,746 & 391 & - & - & - \\
\hline $1998^{1}$ & 3,230 & 2,830 & 400 & - & - & - \\
\hline 1999 & 3,319 & 2,911 & 408 & 305 & 222 & 83 \\
\hline $2000^{1}$ & 3,366 & 2,941 & 424 & - & - & - \\
\hline 2001 & 3,440 & 3,000 & 441 & - & - & - \\
\hline $2002^{1}$ & 3,476 & 3,034 & 442 & - & - & - \\
\hline 2003 & 3,490 & 3,049 & 441 & 311 & 236 & 74 \\
\hline $2004^{1}$ & 3,536 & 3,091 & 445 & - & - & - \\
\hline 2005 & 3,593 & 3,143 & 450 & - & - & - \\
\hline $2006^{1}$ & 3,619 & 3,166 & 453 & - & - & - \\
\hline 2007 & 3,634 & 3,178 & 456 & 327 & 246 & 80 \\
\hline $2008^{1,2}$ & 3,670 & 3,222 & 448 & 388 & 313 & 76 \\
\hline \multicolumn{7}{|c|}{ Projected } \\
\hline $2009^{3}$ & 3,644 & 3,207 & 437 & 353 & 284 & 69 \\
\hline 2010 & 3,668 & 3,240 & 428 & 402 & 333 & 69 \\
\hline 2011 & 3,679 & 3,253 & 426 & 393 & 319 & 74 \\
\hline 2012 & 3,696 & 3,274 & 422 & 400 & 328 & 72 \\
\hline 2013 & 3,725 & 3,306 & 419 & 414 & 342 & 72 \\
\hline 2014 & 3,752 & 3,334 & 419 & 415 & 341 & 74 \\
\hline 2015 & 3,782 & 3,364 & 418 & 419 & 345 & 74 \\
\hline 2016 & 3,814 & 3,395 & 419 & 424 & 349 & 75 \\
\hline 2017 & 3,846 & 3,425 & 420 & 425 & 349 & 76 \\
\hline 2018 & 3,876 & 3,454 & 422 & 427 & 350 & 77 \\
\hline 2019 & 3,910 & 3,485 & 425 & 432 & 354 & 78 \\
\hline 2020 & 3,939 & 3,510 & 429 & 429 & 350 & 79 \\
\hline
\end{tabular}

- Not available.

${ }^{1}$ Since the biennial Private School Universe Survey (PSS) is collected in the fall of odd numbered years, private school numbers for alternate years are estimated based on data from the PSS.

${ }^{2}$ Public and private new teacher hire numbers are estimated using the New Teacher Hires Model. For more information about the New Teacher Hires Model, see appendix A-3.

3 The private school teacher number is an actual number from the 2009-10 PSS.

NOTE: Number of teachers reported in full-time equivalents. Some data have been revised from previously published figures. Detail may not sum to totals because of rounding. Mean absolute percentage errors of selected education statistics can be found in table A-2, appendix A.

SOURCE: U.S. Department of Education, National Center for Education Statistics, Common Core of Data (CCD), "State Nonfiscal Survey of Public Elementary/Secondary Education," 1995-96 through 2008-09; Private School Universe Survey (PSS), selected years, 1995-96 through 2009-10; Schools and Staffing Survey (SASS), "Public School Teacher Questionnaire," 1999-2000 through 2007-08 and "Private School Teacher Questionnaire," 1999-2000 through 2007-08; Elementary and Secondary Teacher Model, 1973-2009; and New Teacher Hires Model, $1988-2007$. (This table was prepared April 2011.) 
Table 17. Actual and projected numbers for the pupil/teacher ratios in elementary and secondary schools, by control of school: Fall 1995 through fall 2020

\begin{tabular}{|c|c|c|c|}
\hline Year & Total & Public & Private \\
\hline \multicolumn{4}{|l|}{ Actual } \\
\hline 1995 & 17.1 & 17.3 & 15.7 \\
\hline $1996^{1}$ & 16.9 & 17.1 & 15.5 \\
\hline 1997 & 16.6 & 16.8 & 15.2 \\
\hline $1998^{1}$ & 16.3 & 16.4 & 15.0 \\
\hline 1999 & 15.9 & 16.1 & 14.7 \\
\hline $2000^{1}$ & 15.9 & 16.0 & 14.5 \\
\hline 2001 & 15.7 & 15.9 & 14.3 \\
\hline $2002^{1}$ & 15.7 & 15.9 & 14.1 \\
\hline 2003 & 15.7 & 15.9 & 13.8 \\
\hline $2004^{1}$ & 15.5 & 15.8 & 13.7 \\
\hline 2005 & 15.4 & 15.6 & 13.5 \\
\hline $2006^{1}$ & 15.3 & 15.6 & 13.2 \\
\hline 2007 & 15.2 & 15.5 & 13.0 \\
\hline $2008^{1}$ & 15.0 & 15.3 & 12.8 \\
\hline \multicolumn{4}{|c|}{ Projected } \\
\hline $2009^{2}$ & 15.0 & 15.4 & 12.5 \\
\hline 2010 & 14.9 & 15.2 & 12.6 \\
\hline 2011 & 14.9 & 15.2 & 12.5 \\
\hline 2012 & 14.9 & 15.2 & 12.5 \\
\hline 2013 & 14.8 & 15.1 & 12.4 \\
\hline 2014 & 14.8 & 15.1 & 12.4 \\
\hline 2015 & 14.8 & 15.1 & 12.4 \\
\hline 2016 & 14.7 & 15.0 & 12.4 \\
\hline 2017 & 14.7 & 15.0 & 12.3 \\
\hline 2018 & 14.7 & 15.0 & 12.3 \\
\hline 2019 & 14.7 & 15.0 & 12.3 \\
\hline 2020 & 14.7 & 15.0 & 12.3 \\
\hline
\end{tabular}

${ }_{1}^{1}$ Since the biennial Private School Universe Survey (PSS) is collected in the fall of odd numbered years, private school numbers for alternate years are estimated based on data from the PSS.

2 The private school teacher number is an actual number from the 2009-10 PSS.

NOTE: The pupil/teacher ratios were derived from tables 1 and 16. Teachers reported in full-time equivalents. Some data have been revised from previously published figures. Mean absolute percentage errors of selected education statistics can be found in table A-2, appendix A.

SOURCE: U.S. Department of Education, National Center for Education Statistics, Common Core of Data (CCD), "State Nonfiscal Survey of Public Elementary/Secondary Education," 1995-96 through 2008-09; Private School Universe Survey (PSS), selected years, 1995-96 through 2009-10; National Elementary and Secondary Enrollment Model, 1972-2008; and Elementary and Secondary Teacher Model, 1973-2009. (This table was prepared February 2011.) 
Table 18. Actual and projected numbers for current expenditures and current expenditures per pupil in fall enrollment for public elementary and secondary education: School years 1995-96 through 2020-21

\begin{tabular}{|c|c|c|c|c|c|}
\hline \multirow[b]{3}{*}{ School year } & \multirow[b]{3}{*}{$\begin{array}{l}\text { Fall enrollment } \\
\text { (in thousands) }\end{array}$} & \multicolumn{4}{|c|}{ Current expenditures } \\
\hline & & \multicolumn{2}{|c|}{ Constant 2008-09 dollars ${ }^{1}$} & \multicolumn{2}{|c|}{ Current dollars } \\
\hline & & $\begin{array}{r}\text { Total } \\
\text { (in billions) }\end{array}$ & $\begin{array}{r}\text { Per pupil } \\
\text { in fall enrollment }\end{array}$ & $\begin{array}{r}\text { Total } \\
\text { (in billions) }\end{array}$ & $\begin{array}{r}\text { Per pupi } \\
\text { in fall enrollment }\end{array}$ \\
\hline \multicolumn{6}{|l|}{ Actual } \\
\hline 1995-96 & 44,840 & $\$ 354.3$ & $\$ 7,902$ & $\$ 255.1$ & $\$ 5,689$ \\
\hline 1996-97 & 45,611 & 364.9 & 8,000 & 270.2 & 5,923 \\
\hline 1997-98 & 46,127 & 378.8 & 8,211 & 285.5 & 6,189 \\
\hline 1998-99 & 46,539 & 395.0 & 8,488 & 302.9 & 6,508 \\
\hline 1999-2000 & 46,857 & 410.6 & 8,762 & 323.9 & 6,912 \\
\hline 2000-01 & 47,204 & 427.0 & 9,047 & 348.4 & 7,380 \\
\hline 2001-02 & 47,672 & 443.7 & 9,308 & 368.4 & 7,727 \\
\hline 2002-03 & 48,183 & 456.8 & 9,480 & 387.6 & 8,044 \\
\hline 2003-04 & 48,540 & 465.1 & 9,583 & 403.4 & 8,310 \\
\hline 2004-05 & 48,795 & 475.8 & 9,751 & 425.0 & 8,711 \\
\hline 2005-06 & 49,113 & 484.5 & 9,865 & 449.1 & 9,145 \\
\hline $2006-07^{2}$ & 49,262 & 501.3 & 10,165 & 476.8 & 9,669 \\
\hline $2007-08^{2}$ & 49,221 & 513.8 & 10,439 & 506.8 & 10,297 \\
\hline \multicolumn{6}{|l|}{ Projected } \\
\hline $2008-09^{3}$ & 49,265 & 513.1 & 10,414 & 513.1 & 10,414 \\
\hline 2009-10 & 49,282 & 511.4 & 10,377 & 516.6 & 10,482 \\
\hline 2010-11 & 49,306 & 514.0 & 10,425 & 526.1 & 10,670 \\
\hline 2011-12 & 49,422 & 515.4 & 10,428 & 536.5 & 10,855 \\
\hline 2012-13 & 49,642 & 518.0 & 10,434 & 550.1 & 11,081 \\
\hline 2013-14 & 49,914 & 525.3 & 10,524 & 569.5 & 11,410 \\
\hline 2014-15 & 50,268 & 540.9 & 10,760 & - & - \\
\hline 2015-16 & 50,659 & 556.6 & 10,987 & - & - \\
\hline 2016-17 & 51,038 & 571.9 & 11,206 & - & - \\
\hline 2017-18 & 51,430 & 585.7 & 11,389 & - & - \\
\hline 2018-19 & 51,803 & 599.2 & 11,567 & - & - \\
\hline 2019-20 & 52,204 & 614.1 & 11,764 & - & - \\
\hline 2020-21 & 52,666 & 627.0 & 11,905 & - & - \\
\hline
\end{tabular}

- Not available.

${ }^{1}$ Based on the Consumer Price Index for all urban consumers, Bureau of Labor Statistics, U.S. Department of Labor. For more detail about CPI, see table B-6 in appendix B.

${ }^{2}$ Fall enrollment pertains only to students for whom finance data were collected. This enrollment count differs slightly from enrollment counts reported on other tables.

${ }^{3}$ The fall enrollment number for 2008-09 is an actual number.

NOTE: Calculations were made using unrounded numbers. Some data have been revised from previously published figures. Mean absolute percentage errors of selected education statistics can be found in table A-2, appendix A.

SOURCE: U.S. Department of Education, National Center for Education Statistics, Common Core of Data (CCD), "State Nonfiscal Survey of Public Elementary/Secondary Education," 1995-96 through 2008-09; "National Public Education Financial Survey," 1995-96 through 2007-08; National Elementary and Secondary Enrollment Model, 1972-2008; and Elementary and Secondary Education Current Expenditures Model, 1969-70 through 2007-08. (This table was prepared February 2011.) 
Table 19. Actual and projected numbers for current expenditures and current expenditures per pupil in average daily attendance (ADA) for public elementary and secondary education: School years 1995-96 through 2020-21

\begin{tabular}{|c|c|c|c|c|c|}
\hline \multirow[b]{3}{*}{ School year } & \multirow[b]{3}{*}{$\begin{array}{r}\text { ADA } \\
\text { (in thousands) }\end{array}$} & \multicolumn{4}{|c|}{ Current expenditures } \\
\hline & & \multicolumn{2}{|c|}{ Constant 2008-09 dollars ${ }^{1}$} & \multicolumn{2}{|c|}{ Current dollars } \\
\hline & & $\begin{array}{r}\text { Total } \\
\text { (in billions) }\end{array}$ & Per pupil in ADA & $\begin{array}{r}\text { Total } \\
\text { (in billions) }\end{array}$ & Per pupil in ADA \\
\hline \multicolumn{6}{|l|}{ Actual } \\
\hline 1995-96 & 41,502 & $\$ 354.3$ & $\$ 8,538$ & $\$ 255.1$ & $\$ 6,147$ \\
\hline 1996-97 & 42,262 & 364.9 & 8,634 & 270.2 & 6,393 \\
\hline 1997-98 & 42,766 & 378.8 & 8,857 & 285.5 & 6,676 \\
\hline 1998-99 & 43,187 & 395.0 & 9,146 & 302.9 & 7,013 \\
\hline 1999-2000 & 43,807 & 410.6 & 9,372 & 323.9 & 7,394 \\
\hline 2000-01 & 44,076 & 427.0 & 9,688 & 348.4 & 7,904 \\
\hline 2001-02 & 44,605 & 443.7 & 9,948 & 368.4 & 8,259 \\
\hline 2002-03 & 45,017 & 456.8 & 10,147 & 387.6 & 8,610 \\
\hline 2003-04 & 45,326 & 465.1 & 10,262 & 403.4 & 8,900 \\
\hline 2004-05 & 45,625 & 475.8 & 10,429 & 425.0 & 9,316 \\
\hline 2005-06 & 45,932 & 484.5 & 10,548 & 449.1 & 9,778 \\
\hline 2006-07 & 46,128 & 501.3 & 10,868 & 476.8 & 10,337 \\
\hline 2007-08 & 46,156 & 513.8 & 11,132 & 506.8 & 10,981 \\
\hline \multicolumn{6}{|l|}{ Projected } \\
\hline 2008-09 & 46,025 & 513.1 & 11,147 & 513.1 & 11,147 \\
\hline 2009-10 & 46,041 & 511.4 & 11,107 & 516.6 & 11,220 \\
\hline 2010-11 & 46,063 & 514.0 & 11,159 & 526.1 & 11,421 \\
\hline 2011-12 & 46,172 & 515.4 & 11,162 & 536.5 & 11,619 \\
\hline 2012-13 & 46,377 & 518.0 & 11,169 & 550.1 & 11,861 \\
\hline 2013-14 & 46,631 & 525.3 & 11,265 & 569.5 & 12,213 \\
\hline 2014-15 & 46,962 & 540.9 & 11,517 & - & - \\
\hline 2015-16 & 47,328 & 556.6 & 11,761 & - & - \\
\hline $2016-17$ & 47,681 & 571.9 & 11,995 & - & - \\
\hline 2017-18 & 48,048 & 585.7 & 12,190 & - & - \\
\hline 2018-19 & 48,396 & 599.2 & 12,381 & - & - \\
\hline 2019-20 & 48,771 & 614.1 & 12,592 & - & - \\
\hline 2020-21 & 49,202 & 627.0 & 12,743 & - & - \\
\hline
\end{tabular}

- Not available.

${ }^{1}$ Based on the Consumer Price Index for all urban consumers, Bureau of Labor Statistics, U.S. Department of Labor. For more detail about CPI, see table B-6 in appendix B.

NOTE: Calculations were made using unrounded numbers. Some data have been revised from previously published figures. Mean absolute percentage errors of selected education statistics can be found in table A-2, appendix A.

SOURCE: U.S. Department of Education, National Center for Education Statistics, Common Core of Data (CCD), "National Public Education Financial Survey," 1995-96 through 2007-08; National Elementary and Secondary Average Daily Attendance Model, 1994-95 through 2007-08; and Elementary and Secondary Education Current Expenditures Model, 1969-70 through 2007-08. (This table was prepared February 2011.) 
Table 20. Actual and projected numbers for total enrollment in all postsecondary degree-granting institutions, by sex, attendance status, and control of institution: Fall 1995 through fall 2020

[In thousands]

\begin{tabular}{|c|c|c|c|c|c|c|c|}
\hline \multirow[b]{2}{*}{ Year } & \multirow[b]{2}{*}{ Total } & \multicolumn{2}{|c|}{ Sex } & \multicolumn{2}{|c|}{ Attendance status } & \multicolumn{2}{|c|}{ Control } \\
\hline & & Men & Women & Full-time & Part-time & Public & Private \\
\hline \multicolumn{8}{|c|}{ Actual } \\
\hline 1995 & 14,262 & 6,343 & 7,919 & 8,129 & 6,133 & 11,092 & 3,169 \\
\hline 1996 & 14,368 & 6,353 & 8,015 & 8,303 & 6,065 & 11,121 & 3,247 \\
\hline 1997 & 14,502 & 6,396 & 8,106 & 8,438 & 6,064 & 11,196 & 3,306 \\
\hline 1998 & 14,507 & 6,369 & 8,138 & 8,563 & 5,944 & 11,138 & 3,369 \\
\hline 1999 & 14,791 & 6,491 & 8,301 & 8,786 & 6,005 & 11,309 & 3,482 \\
\hline 2000 & 15,312 & 6,722 & 8,591 & 9,010 & 6,303 & 11,753 & 3,560 \\
\hline 2001 & 15,928 & 6,961 & 8,967 & 9,448 & 6,480 & 12,233 & 3,695 \\
\hline 2002 & 16,612 & 7,202 & 9,410 & 9,946 & 6,665 & 12,752 & 3,860 \\
\hline 2003 & 16,911 & 7,260 & 9,651 & 10,326 & 6,585 & 12,859 & 4,053 \\
\hline 2004 & 17,272 & 7,387 & 9,885 & 10,610 & 6,662 & 12,980 & 4,292 \\
\hline 2005 & 17,487 & 7,456 & 10,032 & 10,797 & 6,690 & 13,022 & 4,466 \\
\hline 2006 & 17,759 & 7,575 & 10,184 & 10,957 & 6,802 & 13,180 & 4,579 \\
\hline 2007 & 18,248 & 7,816 & 10,432 & 11,270 & 6,978 & 13,491 & 4,757 \\
\hline 2008 & 19,103 & 8,189 & 10,914 & 11,748 & 7,355 & 13,972 & 5,131 \\
\hline 2009 & 20,428 & 8,770 & 11,658 & 12,723 & 7,705 & 14,811 & 5,617 \\
\hline \multicolumn{8}{|c|}{ Projected } \\
\hline 2010 & 20,582 & 8,862 & 11,720 & 12,784 & 7,798 & 14,926 & 5,657 \\
\hline 2011 & 20,688 & 8,896 & 11,793 & 12,854 & 7,835 & 14,998 & 5,691 \\
\hline 2012 & 20,727 & 8,894 & 11,833 & 12,856 & 7,872 & 15,023 & 5,704 \\
\hline 2013 & 20,948 & 8,941 & 12,008 & 12,961 & 7,987 & 15,180 & 5,769 \\
\hline 2014 & 21,320 & 9,028 & 12,291 & 13,158 & 8,162 & 15,445 & 5,875 \\
\hline 2015 & 21,651 & 9,100 & 12,551 & 13,329 & 8,322 & 15,682 & 5,970 \\
\hline 2016 & 21,968 & 9,172 & 12,796 & 13,493 & 8,475 & 15,909 & 6,059 \\
\hline 2017 & 22,251 & 9,239 & 13,013 & 13,641 & 8,610 & 16,115 & 6,137 \\
\hline 2018 & 22,546 & 9,313 & 13,233 & 13,801 & 8,745 & 16,330 & 6,217 \\
\hline 2019 & 22,821 & 9,390 & 13,431 & 13,967 & 8,854 & 16,532 & 6,290 \\
\hline 2020 & 23,016 & 9,450 & 13,566 & 14,089 & 8,927 & 16,676 & 6,340 \\
\hline
\end{tabular}

NOTE: Detail may not sum to totals because of rounding. Some data have been revised from previously published figures. Mean absolute percentage errors of selected education statistics can be found in table A-2, appendix A.

SOURCE: U.S. Department of Education, National Center for Education Statistics, Integrated Postsecondary Education Data System, "Fall

Enrollment Survey" (IPEDS-EF:95-99), and Spring 2001 through Spring 2010; and Enrollment in Degree-Granting Institutions Model, 1980-2009. (This table was prepared January 2011.) 
Table 21. Actual and projected numbers for total enrollment in all postsecondary degree-granting institutions, by age group, sex, and attendance status: Fall 1995 through fall 2020

[In thousands]

\begin{tabular}{|c|c|c|c|c|c|c|c|c|c|c|c|c|c|}
\hline \multirow{2}{*}{$\begin{array}{l}\text { Age group, sex, and } \\
\text { attendance status }\end{array}$} & \multicolumn{13}{|c|}{ Actual } \\
\hline & 1995 & 1996 & 1997 & 1998 & 1999 & 2000 & 2001 & 2002 & 2003 & 2004 & 2005 & 2006 & 2007 \\
\hline Total enrollment & 14,262 & 14,368 & 14,502 & 14,507 & 14,791 & 15,312 & 15,928 & 16,612 & 16,911 & 17,272 & 17,487 & 17,759 & 18,248 \\
\hline 14 to 17 years old & 171 & 183 & 174 & 142 & 137 & 140 & 161 & 162 & 184 & 184 & 210 & 204 & 202 \\
\hline 18 and 19 years old & 2,893 & 3,001 & 3,164 & 3,251 & 3,461 & 3,473 & 3,561 & 3,525 & 3,542 & 3,560 & 3,640 & 3,777 & 3,912 \\
\hline 20 and 21 years old & 2,680 & 2,752 & 2,782 & 2,876 & 2,954 & 3,104 & 3,291 & 3,405 & 3,519 & 3,634 & 3,676 & 3,717 & 3,751 \\
\hline 22 to 24 years old & 2,397 & 2,396 & 2,406 & 2,416 & 2,462 & 2,602 & 2,769 & 3,079 & 3,137 & 3,211 & 3,104 & 3,191 & 3,310 \\
\hline 25 to 29 years old & 2,074 & 2,078 & 2,051 & 1,944 & 1,914 & 1,963 & 2,023 & 2,130 & 2,195 & 2,306 & 2,397 & 2,421 & 2,561 \\
\hline 30 to 34 years old & 1,282 & 1,177 & 1,171 & 1,145 & 1,181 & 1,244 & 1,284 & 1,358 & 1,333 & 1,354 & 1,365 & 1,391 & 1,422 \\
\hline 35 years old and over & 2,765 & 2,780 & 2,754 & 2,733 & 2,683 & 2,786 & 2,839 & 2,954 & 3,001 & 3,022 & 3,095 & 3,058 & 3,091 \\
\hline Men & 6,343 & 6,353 & 6,396 & 6,369 & 6,491 & 6,722 & 6,961 & 7,202 & 7,260 & 7,387 & 7,456 & 7,575 & 7,816 \\
\hline 14 & 71 & 69 & 65 & 56 & 61 & 63 & 66 & 65 & 73 & 73 & 79 & 78 & 86 \\
\hline 18 and 19 years old & 1,329 & 1,363 & 1,443 & 1,470 & 1,570 & 1,559 & 1,608 & 1,590 & 1,580 & 1,569 & 1,608 & 1,690 & 1,767 \\
\hline 21 years old & 1,255 & 1,293 & 1,327 & 1,367 & 1,392 & 1,427 & 1,522 & 1,545 & 1,602 & 1,672 & 1,727 & 1,680 & 1,711 \\
\hline years old & 1,185 & 1,175 & 1,172 & 1,136 & 1,160 & 1,234 & 1,315 & 1,430 & 1,427 & 1,453 & 1,401 & 1,451 & 1,499 \\
\hline $25 t$ & 960 & 975 & 961 & 917 & 863 & 895 & 890 & 941 & 956 & 991 & 1,024 & 1,016 & 1,110 \\
\hline & 545 & 493 & 462 & 474 & 488 & 530 & 527 & 567 & 550 & 550 & 539 & 586 & 598 \\
\hline $35 y$ & 997 & 984 & 967 & 950 & 956 & 1,014 & 1,032 & 1,065 & 1,072 & 1,080 & 1,078 & 1,073 & 1,045 \\
\hline Women & 7,919 & 8,015 & 8,106 & 8,138 & 8,301 & 8,591 & 8,967 & 9,410 & 9,651 & 9,885 & 10,032 & 10,184 & 10,432 \\
\hline & 99 & 114 & 110 & 86 & 76 & 77 & 95 & 97 & 111 & 111 & 131 & 125 & 116 \\
\hline & 1,564 & 1,638 & 1,720 & 1,782 & 1,890 & 914 & 1,953 & 1,935 & 1,962 & 1,991 & 2,031 & 2,087 & 2,145 \\
\hline & 1,425 & 1,4 & 1,455 & 1,5 & 1, & 1,677 & 1,769 & 1,860 & 1,916 & 1,963 & 1,949 & 2,037 & 2,040 \\
\hline & 1,213 & 1,221 & 1,234 & 1,280 & 1,302 & 1,368 & 1,453 & 1,649 & 1,710 & 1,759 & 1,703 & 1,740 & 1,811 \\
\hline 25 & 1,114 & 1,103 & 1,090 & 1,028 & 1,050 & 1,068 & 1,133 & 1,189 & 1,240 & 1,315 & 1,373 & 1,405 & 1,451 \\
\hline & 737 & 684 & 709 & 670 & 693 & 714 & 757 & 791 & 782 & 804 & 826 & 805 & 825 \\
\hline & 1,768 & 1,796 & 1,787 & 1,783 & 1,727 & 1,772 & 1,807 & 1,889 & 1,930 & 1,942 & 2,018 & 1,984 & 2,046 \\
\hline Full- & 8,129 & 8,303 & 8,438 & 8,563 & 8,786 & 9,010 & 9,448 & 9,946 & 10,326 & 10,610 & 10,797 & 10,957 & 11,270 \\
\hline bld & 134 & 138 & 127 & 114 & 117 & 124 & 136 & 135 & 150 & 139 & 155 & 150 & 161 \\
\hline & 2,402 & 2,501 & 2,619 & 2,704 & 2,882 & & 2,932 & 2,924 & & & 65 & & 3,301 \\
\hline & 2,114 & 2,179 & 2,211 & 2,301 & 2,354 & 2,434 & 2,618 & 2,719 & 2,845 & 2,897 & & & 3,036 \\
\hline old & 1,543 & 1,571 & 1,594 & 1,611 & 1,618 & 1,690 & 1,765 & 1,947 & 2,041 & 2,113 & 2,095 & 2,096 & 2,182 \\
\hline & 889 & 902 & 907 & 877 & 860 & 880 & 920 & 1,023 & 1,069 & 1,127 & 1,170 & 1,193 & 1,257 \\
\hline & & & & & & & 456 & & & 23 & & 63 & 540 \\
\hline over & 630 & 617 & 601 & 597 & 587 & 603 & 620 & 694 & 744 & 805 & 809 & 782 & 793 \\
\hline Men & 3,807 & 3,851 & 3,890 & 3,934 & 4,026 & 4,111 & 4,300 & 4,501 & 4,638 & 4,739 & 4,803 & 4,879 & 5,029 \\
\hline & 59 & 58 & 53 & 49 & 53 & 53 & 53 & 52 & 60 & 50 & 55 & 53 & 70 \\
\hline & 1,094 & 1,122 & 1,175 & 1,202 & 1,275 & 1,255 & 1,302 & 1,312 & 1,332 & 1,324 & 1,356 & 1,420 & 1,494 \\
\hline & 993 & 1,015 & 1,053 & 1,104 & 1,130 & 1,133 & 1,217 & 1,241 & 1,304 & 1,353 & 1,392 & 1,366 & 1,383 \\
\hline old & 811 & 807 & 800 & 783 & 795 & 829 & 872 & 945 & 970 & 988 & 972 & 984 & 1,032 \\
\hline & 445 & 458 & 448 & 441 & 406 & 419 & 426 & 477 & 492 & 491 & 503 & 530 & 561 \\
\hline & 171 & 162 & 149 & 151 & 154 & 191 & 196 & 221 & 206 & 229 & 224 & 235 & 224 \\
\hline rs old and over & 234 & 229 & 213 & 203 & 213 & 233 & 235 & 252 & 274 & 305 & 301 & 292 & 266 \\
\hline Women & 4,321 & 4,452 & 4,548 & 4,630 & 4,761 & 4,899 & 5,148 & 5,445 & 5,688 & 5,871 & 5,994 &, 078 & 6,240 \\
\hline & 75 & 80 & 74 & & 64 & 72 & 83 & 82 & 90 & 89 & 100 & 97 & 91 \\
\hline old & 1,308 & 1,379 & 1,444 & 1,502 & 1,607 & 1,604 & 1,630 & 1,612 & 1,659 & 1,682 & 1,709 & 1,761 & 1,807 \\
\hline old & 1,121 & 1,163 & 1,158 & 1,197 & 1,224 & 1,302 & 1,400 & 1,477 & 1,541 & 1,544 & 1,559 & 1,625 & 1,653 \\
\hline 22 to 24 years old & 732 & 765 & 794 & 828 & 823 & 861 & 894 & 1,001 & 1,071 & 1,125 & 1,123 & 1,112 & 1,149 \\
\hline 25 to 29 years old & 444 & 444 & 458 & 436 & 454 & 461 & 494 & 546 & 577 & 636 & 667 & 663 & 696 \\
\hline & 245 & 233 & 230 & 209 & 214 & 229 & 260 & 284 & 280 & 294 & 328 & 329 & 316 \\
\hline 35 years old and over & 396 & 387 & 389 & 394 & 375 & 370 & 386 & 443 & 470 & 501 & 507 & 491 & 528 \\
\hline
\end{tabular}

See notes at end of table. 
Table 21. Actual and projected numbers for total enrollment in all postsecondary degree-granting institutions, by age group, sex, and attendance status: Fall 1995 through fall 2020-Continued

[In thousands]

\begin{tabular}{|c|c|c|c|c|c|c|c|c|c|c|c|c|c|}
\hline \multirow{2}{*}{$\begin{array}{l}\text { Age group, sex, and } \\
\text { attendance status }\end{array}$} & \multicolumn{2}{|c|}{ Actual } & \multicolumn{11}{|c|}{ Projected } \\
\hline & 2008 & 2009 & 2010 & 2011 & 2012 & 2013 & 2014 & 2015 & 2016 & 2017 & 2018 & 2019 & 2020 \\
\hline nrollment & 19,103 & 20,428 & 20,582 & 20,688 & 20,727 & 20,948 & 21,320 & ,651 & 21,968 & 22,251 & 22,546 & 22,821 & 3,016 \\
\hline to 1 & 190 & 209 & 206 & 204 & 202 & 203 & 06 & 211 & 217 & 223 & 232 & 234 & 237 \\
\hline $18 \mathrm{a}$ & 4,015 & 4,295 & 4,341 & 4,295 & 4,244 & 4,232 & 4,264 & 4,282 & ,305 & 4,364 & 4,441 &, 556 & 1,648 \\
\hline & 3,881 & 4,123 & 4,243 & 4,308 & 4,277 & 4,259 & 4,263 & 4,278 & & & & & 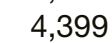 \\
\hline & 485 & 3,682 & 3,713 & 3,771 & 3,860 & 3,958 & 4,044 & & & & & & \\
\hline & 2,748 & 2,971 & 2,975 & 2,980 & 2,987 & & & & & & & & \\
\hline & & & & & & & & & & & & & \\
\hline & 273 & 3,531 & & 3,461 & & & & & 777 & & & & 105 \\
\hline Mer & 8,189 & 8,770 & & & & & 9,028 & & 9,172 & & & &, 450 \\
\hline & 90 & & 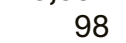 & 97 & 96 & 96 & 96 & 97 & & & & 06 & 07 \\
\hline & 801 & 1,89 & 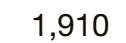 & 007 & 62 & 852 & 860 & & & & & & \\
\hline & & & & & & & & & & & & & \\
\hline & & & & & & & & & & & & & \\
\hline & & & & & & & & & & & & & \\
\hline & & 663 & & & & 728 & 743 & & & 72 & & 91 & 02 \\
\hline & 1,105 & ,165 & & 1,140 & & & & & & & & & ,257 \\
\hline & 80 & & & & & & 12,291 & & & & & & \\
\hline & & & & & & & & & & & & & \\
\hline & 2,2 & 2 & & 2,4 & & & & & & & & & \\
\hline & & & & & & & & & & & & & \\
\hline & & & & & & & & & & & & & \\
\hline & & & & & & & & & & & & & \\
\hline & 871 & & 010 & & & & & & & & & & ,238 \\
\hline & & & & & & & & & & & & & \\
\hline & & 3 & 4 & 4 & & & & & & & & & \\
\hline & & & & & & & 69 & 4 & 79 & 34 & & 94 & 96 \\
\hline & & & & & & & & & & & & & \\
\hline & & & & & & & & & & & & & \\
\hline & & & & & & & & & & & & & 303 \\
\hline & & & & & & & & & & & & & ,704 \\
\hline & & & & & & & & & & & & & \\
\hline & & & & & & & & & & & & & \\
\hline & 5,234 & 5,671 & 689 & 5,709 & 705 & 5,730 & 787 & 834 & 883 & 927 & 978 & 036 & 085 \\
\hline & & & & & & & & & & & & & \\
\hline & & & & & & & & & & & & & \\
\hline & & & & & & & & & & & & & \\
\hline & & & & & & & & & & & & & 307 \\
\hline & & & & & & & & & & & & & \\
\hline & & & & & & & & & & & & & 317 \\
\hline & & & & & & & & 97 & 3 & 09 & 16 & 22 & 326 \\
\hline &, 513 & , & & & & ,232 & 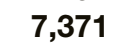 & & & & & & 004 \\
\hline & & & & & & & & & & & & & \\
\hline & 1,853 & $2, \mathrm{C}$ & & & & & 8 & & & & & & 2,244 \\
\hline & 1,679 & & & & & & & & & & & & 1,955 \\
\hline & 1,241 & & & & & & 1,456 & & & & & & 196 \\
\hline & & & & & & & & & & & & & \\
\hline & 31 & & 3 & & & 3 & & 3 & & 404 & & 423 & 430 \\
\hline 35 years old and over & 589 & 670 & 636 & 634 & 632 & 643 & 664 & 684 & 705 & 725 & 745 & 760 & 770 \\
\hline
\end{tabular}

See notes at end of table. 
Table 21. Actual and projected numbers for total enrollment in all postsecondary degree-granting institutions, by age group, sex, and attendance status: Fall 1995 through fall 2020-Continued

[In thousands]

\begin{tabular}{|c|c|c|c|c|c|c|c|c|c|c|c|c|c|}
\hline \multirow{2}{*}{$\begin{array}{l}\text { Age group, sex, and } \\
\text { attendance status }\end{array}$} & \multicolumn{13}{|c|}{ Actual } \\
\hline & 1995 & 1996 & 1997 & 1998 & 1999 & 2000 & 2001 & 2002 & 2003 & 2004 & 2005 & 2006 & 2007 \\
\hline Part-time, total & 6,133 & 6,065 & 6,064 & 5,944 & 6,005 & 6,303 & 6,480 & 6,665 & 6,585 & 6,662 & 6,690 & 6,802 & 6,978 \\
\hline 14 to 17 years old & 36 & 45 & 47 & 28 & 20 & 16 & 25 & 28 & 34 & 45 & 55 & 53 & 41 \\
\hline 18 and 19 years old & 491 & 500 & 545 & 547 & 578 & 614 & 629 & 601 & 550 & 554 & 574 & 596 & 610 \\
\hline 20 and 21 years old & 566 & 573 & 571 & 575 & 600 & 670 & 674 & 686 & 674 & 737 & 725 & 726 & 715 \\
\hline 22 to 24 years old & 854 & 825 & 812 & 805 & 845 & 912 & 1,003 & 1,132 & 1,097 & 1,098 & 1,009 & 1,096 & 1,128 \\
\hline 25 to 29 years old & 1,184 & 1,176 & 1,144 & 1,067 & 1,054 & 1,083 & 1,103 & 1,107 & 1,126 & 1,179 & 1,227 & 1,228 & 1,304 \\
\hline 30 to 34 years old & 866 & 782 & 793 & 785 & 812 & 825 & 828 & 852 & 847 & 832 & 814 & 828 & 882 \\
\hline 35 years old and over & 2,135 & 2,164 & 2,153 & 2,136 & 2,096 & 2,184 & 2,219 & 2,260 & 2,258 & 2,217 & 2,287 & 2,275 & 2,297 \\
\hline Men & 2,535 & 2,502 & 2,506 & 2,436 & 2,465 & 2,611 & 2,661 & 2,701 & 2,622 & 2,648 & 2,653 & 2,695 & 2,786 \\
\hline 14 to 17 years old & 13 & 11 & 11 & 7 & 8 & 10 & 13 & 12 & 13 & 23 & 24 & 25 & 17 \\
\hline 18 and 19 years old & 235 & 241 & 268 & 267 & 295 & 304 & 307 & 278 & 248 & 245 & 252 & 270 & 273 \\
\hline 20 and 21 years old & 262 & 277 & 274 & 262 & 262 & 294 & 305 & 304 & 298 & 319 & 335 & 314 & 328 \\
\hline 22 to 24 years old & 373 & 369 & 372 & 353 & 365 & 405 & 444 & 485 & 457 & 465 & 429 & 467 & 467 \\
\hline 25 to 29 years old & 515 & 517 & 513 & 476 & 457 & 476 & 464 & 464 & 463 & 500 & 521 & 486 & 549 \\
\hline 30 to 34 years old & 375 & 331 & 313 & 323 & 334 & 339 & 331 & 346 & 344 & 322 & 315 & 351 & 373 \\
\hline 35 years old and over & 763 & 755 & 754 & 747 & 743 & 782 & 797 & 813 & 798 & 775 & 776 & 781 & 779 \\
\hline Women & 3,598 & 3,563 & 3,559 & 3,508 & 3,540 & 3,692 & 3,820 & 3,964 & 3,963 & 4,014 & 4,038 & 4,106 & 4,192 \\
\hline 14 to 17 years old & 24 & 34 & 36 & 22 & 12 & 5 & 11 & 15 & 21 & 22 & 31 & 28 & 24 \\
\hline 18 and 19 years old & 256 & 259 & 276 & 280 & 283 & 310 & 323 & 323 & 302 & 310 & 322 & 326 & 337 \\
\hline 20 and 21 years old & 304 & 295 & 297 & 313 & 338 & 376 & 369 & 382 & 375 & 419 & 390 & 412 & 387 \\
\hline 22 to 24 years old & 481 & 456 & 441 & 452 & 479 & 507 & 559 & 647 & 639 & 633 & 580 & 628 & 662 \\
\hline 25 to 29 years old & 670 & 659 & 632 & 591 & 596 & 607 & 639 & 643 & 663 & 679 & 706 & 742 & 755 \\
\hline 30 to 34 years old & 491 & 451 & 480 & 461 & 479 & 485 & 496 & 507 & 502 & 510 & 499 & 477 & 509 \\
\hline 35 years old and over & 1,372 & 1,409 & 1,398 & 1,389 & 1,353 & 1,402 & 1,422 & 1,447 & 1,460 & 1,441 & 1,511 & 1,494 & 1,518 \\
\hline
\end{tabular}

See notes at end of table. 
Table 21. Actual and projected numbers for total enrollment in all postsecondary degree-granting institutions, by age group, sex, and attendance status: Fall 1995 through fall 2020-Continued

[In thousands]

\begin{tabular}{|c|c|c|c|c|c|c|c|c|c|c|c|c|c|}
\hline \multirow{2}{*}{$\begin{array}{l}\text { Age group, sex, and } \\
\text { attendance status }\end{array}$} & \multicolumn{2}{|c|}{ Actual } & \multicolumn{11}{|c|}{ Projected } \\
\hline & 2008 & 2009 & 2010 & 2011 & 2012 & 2013 & 2014 & 2015 & 2016 & 2017 & 2018 & 2019 & 2020 \\
\hline Part-time, total & 7,355 & 7,705 & 7,798 & 7,835 & 7,872 & 7,987 & 8,162 & 8,322 & 8,475 & 8,610 & 8,745 & 8,854 & 8,927 \\
\hline 14 to 17 years old & 33 & 38 & 37 & 37 & 36 & 36 & 37 & 37 & 38 & 39 & 40 & 41 & 41 \\
\hline 18 and 19 years old & 616 & 631 & 636 & 629 & 621 & 617 & 619 & 619 & 619 & 626 & 636 & 651 & 665 \\
\hline 20 and 21 years old & 761 & 781 & 826 & 838 & 833 & 827 & 825 & 824 & 826 & 824 & 823 & 830 & 839 \\
\hline 22 to 24 years old & 1,149 & 1,121 & 1,171 & 1,187 & 1,215 & 1,247 & 1,275 & 1,285 & 1,288 & 1,288 & 1,289 & 1,285 & 1,280 \\
\hline 25 to 29 years old & 1,425 & 1,546 & 1,539 & 1,542 & 1,546 & 1,574 & 1,624 & 1,682 & 1,735 & 1,776 & 1,804 & 1,812 & 1,799 \\
\hline 30 to 34 years old & 962 & 1,029 & 1,032 & 1,060 & 1,083 & 1,113 & 1,147 & 1,175 & 1,200 & 1,222 & 1,247 & 1,271 & 1,293 \\
\hline 35 years old and over & 2,409 & 2,560 & 2,557 & 2,542 & 2,537 & 2,572 & 2,635 & 2,700 & 2,768 & 2,835 & 2,906 & 2,965 & 3,009 \\
\hline Men & 2,955 & 3,099 & 3,172 & 3,186 & 3,189 & 3,211 & 3,242 & 3,266 & 3,289 & 3,312 & 3,336 & 3,354 & 3,365 \\
\hline 14 to 17 years old & 23 & 27 & 24 & 24 & 24 & 24 & 24 & 24 & 24 & 25 & 25 & 26 & 26 \\
\hline 18 and 19 years old & 256 & 250 & 264 & 261 & 257 & 254 & 253 & 251 & 249 & 251 & 253 & 258 & 262 \\
\hline 20 and 21 years old & 356 & 398 & 414 & 420 & 415 & 411 & 407 & 404 & 402 & 398 & 396 & 397 & 401 \\
\hline 22 to 24 years old & 482 & 454 & 480 & 486 & 496 & 506 & 512 & 511 & 507 & 503 & 500 & 495 & 491 \\
\hline 25 to 29 years old & 604 & 710 & 709 & 709 & 709 & 715 & 729 & 746 & 761 & 773 & 780 & 778 & 770 \\
\hline 30 to 34 years old & 403 & 397 & 420 & 431 & 439 & 447 & 455 & 460 & 465 & 468 & 473 & 479 & 485 \\
\hline 35 years old and over & 830 & 864 & 860 & 854 & 850 & 853 & 862 & 870 & 881 & 894 & 909 & 921 & 931 \\
\hline Women & 4,401 & 4,606 & 4,625 & 4,648 & 4,682 & 4,776 & 4,921 & 5,056 & 5,185 & 5,299 & 5,409 & 5,500 & 5,562 \\
\hline 14 to 17 years old & 11 & 11 & 13 & 13 & 13 & 13 & 13 & 13 & 14 & 14 & 15 & 15 & 15 \\
\hline 18 and 19 years old & 361 & 381 & 372 & 368 & 364 & 363 & 366 & 368 & 370 & 375 & 382 & 393 & 403 \\
\hline 20 and 21 years old & 405 & 382 & 412 & 418 & 417 & 416 & 418 & 420 & 424 & 426 & 427 & 433 & 439 \\
\hline 22 to 24 years old & 667 & 667 & 691 & 701 & 718 & 741 & 763 & 774 & 781 & 785 & 789 & 790 & 789 \\
\hline 25 to 29 years old & 820 & 836 & 830 & 832 & 838 & 859 & 896 & 936 & 973 & 1,003 & 1,024 & 1,033 & 1,030 \\
\hline 30 to 34 years old & 558 & 632 & 611 & 629 & 644 & 666 & 692 & 715 & 736 & 754 & 774 & 792 & 808 \\
\hline 35 years old and over & 1,579 & 1,696 & 1,697 & 1,688 & 1,688 & 1,718 & 1,773 & 1,829 & 1,887 & 1,941 & 1,997 & 2,044 & 2,078 \\
\hline
\end{tabular}

NOTE: Detail may not sum to totals because of rounding. Some data have been revised from previously published figures. Data by age are based on the distribution by age from the Census Bureau. For additional information see section A.4 in appendix A. Mean absolute percentage errors of selected education statistics can be found in table A-2, appendix A.

SOURCE: U.S. Department of Education, National Center for Education Statistics, Integrated Postsecondary Education Data System, "Fall

Enrollment Survey" (IPEDS-EF:95-99), and Spring 2001 through Spring 2010; Enrollment in Degree-Granting Institutions Model, 1980-2009; and U.S. Department of Commerce, Census Bureau, Current Population Reports, "Social and Economic Characteristics of Students," various years. (This table was prepared February 2011.) 
Table 22. Actual and projected numbers for enrollment in all postsecondary degree-granting institutions, by sex and attendance status: Fall 1995 through fall 2020

[In thousands]

\begin{tabular}{|c|c|c|c|c|c|}
\hline \multirow[b]{2}{*}{ Year } & \multirow[b]{2}{*}{ Total } & \multicolumn{2}{|c|}{ Men } & \multicolumn{2}{|c|}{ Women } \\
\hline & & Full-time & Part-time & Full-time & Part-time \\
\hline \multicolumn{6}{|c|}{ Actual } \\
\hline 1995 & 14,262 & 3,807 & 2,535 & 4,321 & 3,598 \\
\hline 1996 & 14,368 & 3,851 & 2,502 & 4,452 & 3,563 \\
\hline 1997 & 14,502 & 3,890 & 2,506 & 4,548 & 3,559 \\
\hline 1998 & 14,507 & 3,934 & 2,436 & 4,630 & 3,508 \\
\hline 1999 & 14,791 & 4,026 & 2,465 & 4,761 & 3,540 \\
\hline 2000 & 15,312 & 4,111 & 2,611 & 4,899 & 3,692 \\
\hline 2001 & 15,928 & 4,300 & 2,661 & 5,148 & 3,820 \\
\hline 2002 & 16,612 & 4,501 & 2,701 & 5,445 & 3,964 \\
\hline 2003 & 16,911 & 4,638 & 2,622 & 5,688 & 3,963 \\
\hline 2004 & 17,272 & 4,739 & 2,648 & 5,871 & 4,014 \\
\hline 2005 & 17,487 & 4,803 & 2,653 & 5,994 & 4,038 \\
\hline 2006 & 17,759 & 4,879 & 2,695 & 6,078 & 4,106 \\
\hline 2007 & 18,248 & 5,029 & 2,786 & 6,240 & 4,192 \\
\hline 2008 & 19,103 & 5,234 & 2,955 & 6,513 & 4,401 \\
\hline 2009 & 20,428 & 5,671 & 3,099 & 7,052 & 4,606 \\
\hline \multicolumn{6}{|c|}{ Projected } \\
\hline 2010 & 20,582 & 5,689 & 3,172 & 7,095 & 4,625 \\
\hline 2011 & 20,688 & 5,709 & 3,186 & 7,145 & 4,648 \\
\hline 2012 & 20,727 & 5,705 & 3,189 & 7,151 & 4,682 \\
\hline 2013 & 20,948 & 5,730 & 3,211 & 7,232 & 4,776 \\
\hline 2014 & 21,320 & 5,787 & 3,242 & 7,371 & 4,921 \\
\hline 2015 & 21,651 & 5,834 & 3,266 & 7,495 & 5,056 \\
\hline 2016 & 21,968 & 5,883 & 3,289 & 7,610 & 5,185 \\
\hline 2017 & 22,251 & 5,927 & 3,312 & 7,714 & 5,299 \\
\hline 2018 & 22,546 & 5,978 & 3,336 & 7,824 & 5,409 \\
\hline 2019 & 22,821 & 6,036 & 3,354 & 7,931 & 5,500 \\
\hline 2020 & 23,016 & 6,085 & 3,365 & 8,004 & 5,562 \\
\hline
\end{tabular}

NOTE: Detail may not sum to totals because of rounding. Some data have been revised from previously published figures. Mean absolute percentage errors of selected education statistics can be found in table A-2, appendix A.

SOURCE: U.S. Department of Education, National Center for Education Statistics, Integrated Postsecondary Education Data System, "Fall

Enrollment Survey" (IPEDS-EF:95-99), and Spring 2001 through Spring 2010; and Enrollment in Degree-Granting Institutions Model, 1980-2009. (This table was prepared February 2011.) 
Table 23. Actual and projected numbers for enrollment in public 4-year postsecondary degree-granting institutions, by sex and attendance status: Fall 1995 through fall 2020

[In thousands]

\begin{tabular}{|c|c|c|c|c|c|}
\hline \multirow[b]{2}{*}{ Year } & \multirow[b]{2}{*}{ Total } & \multicolumn{2}{|c|}{ Men } & \multicolumn{2}{|c|}{ Women } \\
\hline & & Full-time & Part-time & Full-time & Part-time \\
\hline \multicolumn{6}{|c|}{ Actual } \\
\hline 1995 & 5,815 & 1,951 & 720 & 2,134 & 1,009 \\
\hline 1996 & 5,806 & 1,943 & 703 & 2,163 & 997 \\
\hline 1997 & 5,835 & 1,951 & 687 & 2,214 & 984 \\
\hline 1998 & 5,892 & 1,959 & 685 & 2,260 & 988 \\
\hline 1999 & 5,970 & 1,984 & 686 & 2,309 & 991 \\
\hline 2000 & 6,055 & 2,009 & 683 & 2,363 & 1,001 \\
\hline 2001 & 6,236 & 2,082 & 687 & 2,450 & 1,017 \\
\hline 2002 & 6,482 & 2,167 & 706 & 2,557 & 1,052 \\
\hline 2003 & 6,649 & 2,225 & 713 & 2,639 & 1,072 \\
\hline 2004 & 6,737 & 2,260 & 717 & 2,684 & 1,076 \\
\hline 2005 & 6,838 & 2,295 & 724 & 2,726 & 1,091 \\
\hline 2006 & 6,955 & 2,339 & 740 & 2,765 & 1,111 \\
\hline 2007 & 7,167 & 2,418 & 773 & 2,827 & 1,149 \\
\hline 2008 & 7,332 & 2,488 & 789 & 2,890 & 1,165 \\
\hline 2009 & 7,709 & 2,626 & 833 & 3,024 & 1,226 \\
\hline \multicolumn{6}{|c|}{ Projected } \\
\hline 2010 & 7,771 & 2,642 & 848 & 3,046 & 1,235 \\
\hline 2011 & 7,817 & 2,653 & 852 & 3,071 & 1,242 \\
\hline 2012 & 7,833 & 2,652 & 854 & 3,074 & 1,252 \\
\hline 2013 & 7,913 & 2,664 & 862 & 3,109 & 1,279 \\
\hline 2014 & 8,048 & 2,690 & 872 & 3,166 & 1,320 \\
\hline 2015 & 8,167 & 2,711 & 880 & 3,217 & 1,359 \\
\hline 2016 & 8,280 & 2,733 & 888 & 3,264 & 1,395 \\
\hline 2017 & 8,379 & 2,752 & 895 & 3,306 & 1,427 \\
\hline 2018 & 8,483 & 2,774 & 902 & 3,349 & 1,458 \\
\hline 2019 & 8,581 & 2,800 & 907 & 3,393 & 1,482 \\
\hline 2020 & 8,651 & 2,821 & 909 & 3,423 & 1,498 \\
\hline
\end{tabular}

NOTE: Detail may not sum to totals because of rounding. Some data have been revised from previously published figures. Mean absolute percentage errors of selected education statistics can be found in table A-2, appendix A.

SOURCE: U.S. Department of Education, National Center for Education Statistics, Integrated Postsecondary Education Data System, "Fall

Enrollment Survey" (IPEDS-EF:95-99), and Spring 2001 through Spring 2010; and Enrollment in Degree-Granting Institutions Model, 1980-2009. (This table was prepared February 2011.) 
Table 24. Actual and projected numbers for enrollment in public 2-year postsecondary degree-granting institutions, by sex and attendance status: Fall 1995 through fall 2020

[In thousands]

\begin{tabular}{|c|c|c|c|c|c|}
\hline \multirow[b]{2}{*}{ Year } & \multirow[b]{2}{*}{ Total } & \multicolumn{2}{|c|}{ Men } & \multicolumn{2}{|c|}{ Women } \\
\hline & & Full-time & Part-time & Full-time & Part-time \\
\hline \multicolumn{6}{|c|}{ Actual } \\
\hline 1995 & 5,278 & 819 & 1,417 & 1,022 & 2,020 \\
\hline 1996 & 5,314 & 833 & 1,423 & 1,039 & 2,019 \\
\hline 1997 & 5,361 & 842 & 1,444 & 1,049 & 2,026 \\
\hline 1998 & 5,246 & 841 & 1,383 & 1,040 & 1,981 \\
\hline 1999 & 5,339 & 868 & 1,404 & 1,063 & 2,005 \\
\hline 2000 & 5,697 & 891 & 1,549 & 1,109 & 2,148 \\
\hline 2001 & 5,997 & 962 & 1,596 & 1,194 & 2,245 \\
\hline 2002 & 6,270 & 1,035 & 1,605 & 1,299 & 2,332 \\
\hline 2003 & 6,209 & 1,060 & 1,515 & 1,346 & 2,288 \\
\hline 2004 & 6,244 & 1,065 & 1,518 & 1,360 & 2,300 \\
\hline 2005 & 6,184 & 1,055 & 1,514 & 1,332 & 2,283 \\
\hline 2006 & 6,225 & 1,067 & 1,533 & 1,325 & 2,300 \\
\hline 2007 & 6,324 & 1,099 & 1,568 & 1,343 & 2,314 \\
\hline 2008 & 6,640 & 1,152 & 1,672 & 1,396 & 2,420 \\
\hline 2009 & 7,101 & 1,318 & 1,733 & 1,563 & 2,488 \\
\hline \multicolumn{6}{|c|}{ Projected } \\
\hline 2010 & 7,155 & 1,307 & 1,784 & 1,569 & 2,495 \\
\hline 2011 & 7,181 & 1,309 & 1,791 & 1,575 & 2,506 \\
\hline 2012 & 7,190 & 1,305 & 1,790 & 1,573 & 2,522 \\
\hline 2013 & 7,266 & 1,308 & 1,799 & 1,590 & 2,569 \\
\hline 2014 & 7,397 & 1,320 & 1,813 & 1,621 & 2,643 \\
\hline 2015 & 7,515 & 1,330 & 1,824 & 1,649 & 2,712 \\
\hline 2016 & 7,629 & 1,341 & 1,834 & 1,676 & 2,778 \\
\hline 2017 & 7,736 & 1,352 & 1,845 & 1,703 & 2,836 \\
\hline 2018 & 7,847 & 1,366 & 1,857 & 1,731 & 2,893 \\
\hline 2019 & 7,951 & 1,383 & 1,867 & 1,760 & 2,941 \\
\hline 2020 & 8,025 & 1,397 & 1,874 & 1,779 & 2,975 \\
\hline
\end{tabular}

NOTE: Detail may not sum to totals because of rounding. Some data have been revised from previously published figures. Mean absolute percentage errors of selected education statistics can be found in table A-2, appendix A.

SOURCE: U.S. Department of Education, National Center for Education Statistics, Integrated Postsecondary Education Data System, "Fall

Enrollment Survey" (IPEDS-EF:95-99), and Spring 2001 through Spring 2010; and Enrollment in Degree-Granting Institutions Model, 1980-2009. (This table was prepared January 2011.) 
Table 25. Actual and projected numbers for enrollment in private 4-year postsecondary degree-granting institutions, by sex and attendance status: Fall 1995 through fall 2020

[In thousands]

\begin{tabular}{|c|c|c|c|c|c|}
\hline \multirow[b]{2}{*}{ Year } & \multirow[b]{2}{*}{ Total } & \multicolumn{2}{|c|}{ Men } & \multicolumn{2}{|c|}{ Women } \\
\hline & & Full-time & Part-time & Full-time & Part-time \\
\hline \multicolumn{6}{|c|}{ Actual } \\
\hline 1995 & 2,955 & 978 & 364 & 1,089 & 523 \\
\hline 1996 & 2,998 & 991 & 356 & 1,133 & 518 \\
\hline 1997 & 3,061 & 1,008 & 360 & 1,170 & 523 \\
\hline 1998 & 3,126 & 1,038 & 353 & 1,220 & 514 \\
\hline 1999 & 3,229 & 1,073 & 360 & 1,276 & 519 \\
\hline 2000 & 3,308 & 1,107 & 365 & 1,315 & 522 \\
\hline 2001 & 3,441 & 1,151 & 365 & 1,389 & 536 \\
\hline 2002 & 3,601 & 1,199 & 377 & 1,468 & 557 \\
\hline 2003 & 3,768 & 1,250 & 382 & 1,561 & 574 \\
\hline 2004 & 3,990 & 1,313 & 400 & 1,670 & 607 \\
\hline 2005 & 4,162 & 1,354 & 402 & 1,774 & 632 \\
\hline 2006 & 4,285 & 1,381 & 411 & 1,830 & 664 \\
\hline 2007 & 4,464 & 1,422 & 433 & 1,911 & 698 \\
\hline 2008 & 4,800 & 1,496 & 480 & 2,041 & 782 \\
\hline 2009 & 5,197 & 1,596 & 518 & 2,228 & 855 \\
\hline \multicolumn{6}{|c|}{ Projected } \\
\hline 2010 & 5,238 & 1,611 & 526 & 2,241 & 860 \\
\hline 2011 & 5,271 & 1,618 & 528 & 2,259 & 865 \\
\hline 2012 & 5,285 & 1,619 & 530 & 2,264 & 872 \\
\hline 2013 & 5,346 & 1,628 & 534 & 2,292 & 891 \\
\hline 2014 & 5,445 & 1,646 & 541 & 2,338 & 920 \\
\hline 2015 & 5,533 & 1,661 & 546 & 2,378 & 948 \\
\hline 2016 & 5,616 & 1,676 & 551 & 2,415 & 973 \\
\hline 2017 & 5,688 & 1,689 & 556 & 2,447 & 996 \\
\hline 2018 & 5,761 & 1,702 & 561 & 2,480 & 1,018 \\
\hline 2019 & 5,827 & 1,717 & 564 & 2,511 & 1,035 \\
\hline 2020 & 5,873 & 1,729 & 566 & 2,532 & 1,046 \\
\hline
\end{tabular}

NOTE: Detail may not sum to totals because of rounding. Some data have been revised from previously published figures. Mean absolute percentage errors of selected education statistics can be found in table A-2, appendix A.

SOURCE: U.S. Department of Education, National Center for Education Statistics, Integrated Postsecondary Education Data System, "Fall

Enrollment Survey" (IPEDS-EF:95-99), and Spring 2001 through Spring 2010; and Enrollment in Degree-Granting Institutions Model, 1980-2009. (This table was prepared February 2011.) 
Table 26. Actual and projected numbers for enrollment in private 2-year postsecondary degree-granting institutions, by sex and attendance status: Fall 1995 through fall 2020

[In thousands]

\begin{tabular}{|c|c|c|c|c|c|}
\hline \multirow[b]{2}{*}{ Year } & \multirow[b]{2}{*}{ Total } & \multicolumn{2}{|c|}{ Men } & \multicolumn{2}{|c|}{ Women } \\
\hline & & Full-time & Part-time & Full-time & Part-time \\
\hline \multicolumn{6}{|c|}{ Actual } \\
\hline 1995 & 215 & 60 & 33 & 77 & 45 \\
\hline 1996 & 249 & 84 & 19 & 117 & 29 \\
\hline 1997 & 245 & 89 & 14 & 115 & 26 \\
\hline 1998 & 243 & 95 & 14 & 109 & 25 \\
\hline 1999 & 253 & 101 & 15 & 112 & 25 \\
\hline 2000 & 251 & 105 & 13 & 112 & 21 \\
\hline 2001 & 254 & 105 & 12 & 114 & 22 \\
\hline 2002 & 259 & 101 & 13 & 122 & 23 \\
\hline 2003 & 285 & 103 & 13 & 142 & 28 \\
\hline 2004 & 302 & 101 & 13 & 156 & 31 \\
\hline 2005 & 304 & 99 & 12 & 161 & 32 \\
\hline 2006 & 293 & 93 & 11 & 159 & 30 \\
\hline 2007 & 294 & 91 & 12 & 159 & 31 \\
\hline 2008 & 331 & 98 & 14 & 186 & 33 \\
\hline 2009 & 420 & 131 & 16 & 237 & 36 \\
\hline \multicolumn{6}{|c|}{ Projected } \\
\hline 2010 & 419 & 130 & 16 & 238 & 35 \\
\hline 2011 & 420 & 130 & 16 & 239 & 35 \\
\hline 2012 & 420 & 129 & 16 & 239 & 36 \\
\hline 2013 & 423 & 130 & 16 & 241 & 36 \\
\hline 2014 & 430 & 131 & 16 & 246 & 37 \\
\hline 2015 & 437 & 132 & 16 & 250 & 38 \\
\hline 2016 & 443 & 133 & 16 & 254 & 39 \\
\hline 2017 & 449 & 134 & 16 & 259 & 40 \\
\hline 2018 & 455 & 136 & 16 & 263 & 41 \\
\hline 2019 & 462 & 137 & 16 & 267 & 42 \\
\hline 2020 & 467 & 139 & 16 & 270 & 42 \\
\hline
\end{tabular}

NOTE: Detail may not sum to totals because of rounding. Some data have been revised from previously published figures. Mean absolute percentage errors of selected education statistics can be found in table A-2, appendix A.

SOURCE: U.S. Department of Education, National Center for Education Statistics, Integrated Postsecondary Education Data System, "Fall

Enrollment Survey" (IPEDS-EF:95-99), and Spring 2001 through Spring 2010; and Enrollment in Degree-Granting Institutions Model, 1980-2009. (This table was prepared January 2011.) 
Table 27. Actual and projected numbers for undergraduate enrollment in all postsecondary degree-granting institutions, by sex, attendance status, and control of institution: Fall 1995 through fall 2020

[In thousands]

\begin{tabular}{|c|c|c|c|c|c|c|c|}
\hline \multirow[b]{2}{*}{ Year } & \multirow[b]{2}{*}{ Total } & \multicolumn{2}{|c|}{ Sex } & \multicolumn{2}{|c|}{ Attendance status } & \multicolumn{2}{|c|}{ Control } \\
\hline & & Men & Women & Full-time & Part-time & Public & Private \\
\hline \multicolumn{8}{|c|}{ Actual } \\
\hline 1995 & 12,232 & 5,401 & 6,831 & 7,145 & 5,086 & 9,904 & 2,328 \\
\hline 1996 & 12,327 & 5,421 & 6,906 & 7,299 & 5,028 & 9,935 & 2,392 \\
\hline 1997 & 12,451 & 5,469 & 6,982 & 7,419 & 5,032 & 10,007 & 2,443 \\
\hline 1998 & 12,437 & 5,446 & 6,991 & 7,539 & 4,898 & 9,950 & 2,487 \\
\hline 1999 & 12,681 & 5,559 & 7,122 & 7,735 & 4,946 & 10,110 & 2,571 \\
\hline 2000 & 13,155 & 5,778 & 7,377 & 7,923 & 5,232 & 10,539 & 2,616 \\
\hline 2001 & 13,716 & 6,004 & 7,711 & 8,328 & 5,388 & 10,986 & 2,730 \\
\hline 2002 & 14,257 & 6,192 & 8,065 & 8,734 & 5,523 & 11,433 & 2,824 \\
\hline 2003 & 14,480 & 6,227 & 8,253 & 9,045 & 5,435 & 11,523 & 2,957 \\
\hline 2004 & 14,781 & 6,340 & 8,441 & 9,284 & 5,496 & 11,651 & 3,130 \\
\hline 2005 & 14,964 & 6,409 & 8,555 & 9,446 & 5,518 & 11,698 & 3,266 \\
\hline 2006 & 15,184 & 6,514 & 8,671 & 9,571 & 5,613 & 11,847 & 3,337 \\
\hline 2007 & 15,604 & 6,728 & 8,876 & 9,841 & 5,763 & 12,138 & 3,466 \\
\hline 2008 & 16,366 & 7,067 & 9,299 & 10,255 & 6,111 & 12,591 & 3,775 \\
\hline 2009 & 17,565 & 7,595 & 9,970 & 11,143 & 6,422 & 13,387 & 4,179 \\
\hline \multicolumn{8}{|c|}{ Projected } \\
\hline 2010 & 17,699 & 7,657 & 10,042 & 11,190 & 6,509 & 13,491 & 4,208 \\
\hline 2011 & 17,786 & 7,684 & 10,101 & 11,245 & 6,541 & 13,554 & 4,232 \\
\hline 2012 & 17,801 & 7,675 & 10,125 & 11,231 & 6,570 & 13,567 & 4,234 \\
\hline 2013 & 17,965 & 7,706 & 10,259 & 11,304 & 6,661 & 13,695 & 4,270 \\
\hline 2014 & 18,255 & 7,771 & 10,484 & 11,455 & 6,801 & 13,921 & 4,335 \\
\hline 2015 & 18,512 & 7,824 & 10,688 & 11,585 & 6,927 & 14,121 & 4,391 \\
\hline 2016 & 18,759 & 7,877 & 10,882 & 11,713 & 7,046 & 14,313 & 4,446 \\
\hline 2017 & 18,985 & 7,928 & 11,057 & 11,832 & 7,153 & 14,490 & 4,495 \\
\hline 2018 & 19,226 & 7,989 & 11,237 & 11,968 & 7,259 & 14,678 & 4,549 \\
\hline 2019 & 19,469 & 8,059 & 11,409 & 12,122 & 7,347 & 14,864 & 4,605 \\
\hline 2020 & 19,649 & 8,117 & 11,532 & 12,243 & 7,406 & 15,000 & 4,648 \\
\hline
\end{tabular}

NOTE: Detail may not sum to totals because of rounding. Some data have been revised from previously published figures. Mean absolute percentage errors of selected education statistics can be found in table A-2, appendix A.

SOURCE: U.S. Department of Education, National Center for Education Statistics, Integrated Postsecondary Education Data System, "Fall

Enrollment Survey" (IPEDS-EF:95-99), and Spring 2001 through Spring 2010; and Enrollment in Degree-Granting Institutions Model, 1980-2009. (This table was prepared January 2011.) 
Table 28. Actual and projected numbers for postbaccalaureate enrollment in all postsecondary degree-granting institutions, by sex, attendance status, and control of institution: Fall 1995 through fall 2020

[ln thousands]

\begin{tabular}{|c|c|c|c|c|c|c|c|}
\hline \multirow[b]{2}{*}{ Year } & \multirow[b]{2}{*}{ Total } & \multicolumn{2}{|c|}{ Sex } & \multicolumn{2}{|c|}{ Attendance status } & \multicolumn{2}{|c|}{ Control } \\
\hline & & Men & Women & Full-time & Part-time & Public & Private \\
\hline \multicolumn{8}{|c|}{ Actual } \\
\hline 1995 & 2,030 & 941 & 1,088 & 984 & 1,046 & 1,189 & 841 \\
\hline 1996 & 2,041 & 932 & 1,108 & 1,004 & 1,036 & 1,185 & 855 \\
\hline 1997 & 2,052 & 927 & 1,124 & 1,019 & 1,032 & 1,189 & 863 \\
\hline 1998 & 2,070 & 923 & 1,147 & 1,025 & 1,045 & 1,188 & 882 \\
\hline 1999 & 2,110 & 931 & 1,179 & 1,051 & 1,058 & 1,199 & 911 \\
\hline 2000 & 2,157 & 943 & 1,213 & 1,087 & 1,070 & 1,213 & 943 \\
\hline 2001 & 2,212 & 956 & 1,256 & 1,120 & 1,092 & 1,247 & 965 \\
\hline 2002 & 2,355 & 1,010 & 1,345 & 1,212 & 1,142 & 1,319 & 1,035 \\
\hline 2003 & 2,431 & 1,033 & 1,398 & 1,281 & 1,150 & 1,336 & 1,096 \\
\hline 2004 & 2,491 & 1,047 & 1,444 & 1,326 & 1,165 & 1,330 & 1,162 \\
\hline 2005 & 2,524 & 1,047 & 1,476 & 1,351 & 1,173 & 1,324 & 1,199 \\
\hline 2006 & 2,575 & 1,061 & 1,513 & 1,386 & 1,188 & 1,333 & 1,242 \\
\hline 2007 & 2,644 & 1,088 & 1,556 & 1,429 & 1,215 & 1,353 & 1,291 \\
\hline 2008 & 2,737 & 1,122 & 1,615 & 1,493 & 1,244 & 1,381 & 1,356 \\
\hline 2009 & 2,862 & 1,174 & 1,688 & 1,579 & 1,283 & 1,424 & 1,438 \\
\hline \multicolumn{8}{|c|}{ Projected } \\
\hline 2010 & 2,884 & 1,205 & 1,678 & 1,594 & 1,289 & 1,435 & 1,449 \\
\hline 2011 & 2,903 & 1,212 & 1,691 & 1,609 & 1,294 & 1,445 & 1,459 \\
\hline 2012 & 2,927 & 1,219 & 1,707 & 1,625 & 1,302 & 1,456 & 1,471 \\
\hline 2013 & 2,984 & 1,235 & 1,748 & 1,658 & 1,326 & 1,484 & 1,500 \\
\hline 2014 & 3,065 & 1,257 & 1,807 & 1,703 & 1,362 & 1,525 & 1,540 \\
\hline 2015 & 3,140 & 1,277 & 1,863 & 1,744 & 1,396 & 1,562 & 1,578 \\
\hline 2016 & 3,209 & 1,295 & 1,913 & 1,781 & 1,428 & 1,596 & 1,613 \\
\hline 2017 & 3,267 & 1,311 & 1,956 & 1,809 & 1,458 & 1,625 & 1,642 \\
\hline 2018 & 3,320 & 1,324 & 1,996 & 1,834 & 1,486 & 1,652 & 1,668 \\
\hline 2019 & 3,353 & 1,331 & 2,021 & 1,845 & 1,507 & 1,668 & 1,684 \\
\hline 2020 & 3,368 & 1,333 & 2,034 & 1,847 & 1,521 & 1,676 & 1,692 \\
\hline
\end{tabular}

NOTE: Detail may not sum to totals because of rounding. Some data have been revised from previously published figures. Mean absolute percentage errors of selected education statistics can be found in table A-2, appendix A.

SOURCE: U.S. Department of Education, National Center for Education Statistics, Integrated Postsecondary Education Data System, "Fall Enrollment Survey" (IPEDS-EF:95-99), and Spring 2001 through Spring 2010; and Enrollment in Degree-Granting Institutions Model, 1980-2009. (This table was prepared January 2011.) 
Table 29. Actual and projected numbers for enrollment in all postsecondary degree-granting institutions, by race/ethnicity: Fall 1995 through fall 2020

[In thousands]

\begin{tabular}{|c|c|c|c|c|c|c|c|}
\hline \multirow[b]{2}{*}{ Year } & \multirow[b]{2}{*}{ Total } & \multicolumn{5}{|c|}{ Race/ethnicity } & \multirow[b]{2}{*}{$\begin{array}{r}\text { Nonresident } \\
\text { alien }^{1} \\
\end{array}$} \\
\hline & & White & Black & Hispanic & $\begin{array}{r}\text { Asian/Pacific } \\
\text { Islander }\end{array}$ & $\begin{array}{r}\text { American Indian/ } \\
\text { Alaska Native }\end{array}$ & \\
\hline \multicolumn{8}{|c|}{ Actual } \\
\hline 1995 & 14,262 & 10,311 & 1,474 & 1,094 & 797 & 131 & 454 \\
\hline 1996 & 14,368 & 10,264 & 1,506 & 1,166 & 828 & 138 & 466 \\
\hline 1997 & 14,502 & 10,266 & 1,551 & 1,218 & 859 & 142 & 465 \\
\hline 1998 & 14,507 & 10,179 & 1,583 & 1,257 & 900 & 144 & 444 \\
\hline 1999 & 14,791 & 10,282 & 1,643 & 1,319 & 913 & 145 & 488 \\
\hline 2000 & 15,312 & 10,462 & 1,730 & 1,462 & 978 & 151 & 529 \\
\hline 2001 & 15,928 & 10,775 & 1,850 & 1,561 & 1,019 & 158 & 565 \\
\hline 2002 & 16,612 & 11,140 & 1,979 & 1,662 & 1,074 & 166 & 591 \\
\hline 2003 & 16,911 & 11,281 & 2,068 & 1,716 & 1,076 & 173 & 598 \\
\hline 2004 & 17,272 & 11,423 & 2,165 & 1,810 & 1,109 & 176 & 590 \\
\hline 2005 & 17,487 & 11,495 & 2,215 & 1,882 & 1,134 & 176 & 585 \\
\hline 2006 & 17,759 & 11,572 & 2,280 & 1,964 & 1,165 & 181 & 596 \\
\hline 2007 & 18,248 & 11,756 & 2,383 & 2,076 & 1,218 & 190 & 624 \\
\hline 2008 & 19,103 & 12,089 & 2,584 & 2,273 & 1,303 & 193 & 661 \\
\hline 2009 & 20,428 & 12,731 & 2,920 & 2,547 & 1,338 & 208 & 685 \\
\hline \multicolumn{8}{|c|}{ Projected } \\
\hline 2010 & 20,582 & 12,745 & 2,946 & 2,620 & 1,362 & 208 & 701 \\
\hline 2011 & 20,688 & 12,709 & 2,989 & 2,687 & 1,382 & 206 & 715 \\
\hline 2012 & 20,727 & 12,613 & 3,031 & 2,753 & 1,401 & 204 & 726 \\
\hline 2013 & 20,948 & 12,618 & 3,104 & 2,851 & 1,429 & 204 & 742 \\
\hline 2014 & 21,320 & 12,702 & 3,201 & 2,982 & 1,468 & 205 & 762 \\
\hline 2015 & 21,651 & 12,751 & 3,294 & 3,114 & 1,506 & 205 & 782 \\
\hline 2016 & 21,968 & 12,794 & 3,382 & 3,243 & 1,542 & 205 & 802 \\
\hline 2017 & 22,251 & 12,826 & 3,461 & 3,364 & 1,575 & 205 & 820 \\
\hline 2018 & 22,546 & 12,873 & 3,536 & 3,486 & 1,607 & 206 & 839 \\
\hline 2019 & 22,821 & 12,918 & 3,598 & 3,606 & 1,638 & 205 & 855 \\
\hline 2020 & 23,016 & 12,921 & 3,646 & 3,707 & 1,666 & 205 & 872 \\
\hline
\end{tabular}

1 The racial/ethnic backgrounds of nonresident aliens are not known.

NOTE: Race categories exclude persons of Hispanic ethnicity. Enrollment data in the "race/ethnicity unknown" (all years) and "two or more races" (2008 and 2009 only) categories of the IPEDS "Fall Enrollment Survey" have been prorated to the other racial/ethnic categories at the institutional level. Detail may not sum to totals because of rounding. Mean absolute percentage errors of selected education statistics can be found in table A-2, appendix A.

SOURCE: U.S. Department of Education, National Center for Education Statistics, Integrated Postsecondary Education Data System, "Fall Enrollment Survey" (IPEDS-EF:95-99), and Spring 2001 through Spring 2009; and Enrollment in Degree-Granting Institutions by Race/Ethnicity Model, 19802009. (This table was prepared February 2011.) 
Table 30. Actual and projected numbers for first-time freshmen fall enrollment in all postsecondary degree-granting institutions, by sex: Fall 1995 through fall 2020

\begin{tabular}{|c|c|c|c|}
\hline Year & Total & Men & Women \\
\hline \multicolumn{4}{|c|}{ Actual } \\
\hline 1995 & 2,169 & 1,001 & 1,168 \\
\hline 1996 & 2,274 & 1,047 & 1,228 \\
\hline 1997 & 2,219 & 1,026 & 1,193 \\
\hline 1998 & 2,213 & 1,023 & 1,190 \\
\hline 1999 & 2,352 & 1,092 & 1,260 \\
\hline 2000 & 2,428 & 1,124 & 1,304 \\
\hline 2001 & 2,497 & 1,153 & 1,344 \\
\hline 2002 & 2,571 & 1,171 & 1,400 \\
\hline 2003 & 2,592 & 1,176 & 1,416 \\
\hline 2004 & 2,630 & 1,190 & 1,440 \\
\hline 2005 & 2,657 & 1,200 & 1,457 \\
\hline 2006 & 2,707 & 1,229 & 1,479 \\
\hline 2007 & 2,776 & 1,267 & 1,509 \\
\hline 2008 & 3,025 & 1,389 & 1,635 \\
\hline 2009 & 3,210 & 1,480 & 1,730 \\
\hline \multicolumn{4}{|c|}{ Projected } \\
\hline 2010 & 3,215 & 1,477 & 1,738 \\
\hline 2011 & 3,231 & 1,483 & 1,748 \\
\hline 2012 & 3,233 & 1,481 & 1,752 \\
\hline 2013 & 3,262 & 1,487 & 1,776 \\
\hline 2014 & 3,314 & 1,499 & 1,814 \\
\hline 2015 & 3,359 & 1,510 & 1,850 \\
\hline 2016 & 3,403 & 1,520 & 1,883 \\
\hline 2017 & 3,443 & 1,530 & 1,914 \\
\hline 2018 & 3,486 & 1,541 & 1,945 \\
\hline 2019 & 3,530 & 1,555 & 1,975 \\
\hline 2020 & 3,562 & 1,566 & 1,996 \\
\hline
\end{tabular}

NOTE: Detail may not sum to totals because of rounding. Some data have been revised from previously published figures. Mean absolute percentage errors of selected education statistics can be found in table A-2, appendix A.

SOURCE: U.S. Department of Education, National Center for Education Statistics, Integrated Postsecondary Education Data System, "Fall

Enrollment Survey" (IPEDS-EF:95-99), and Spring 2001 through Spring 2010; and First-Time Freshmen Model, 1975-2009. (This table was prepared March 2011.) 
Table 31. Actual and projected numbers for full-time-equivalent enrollment in all postsecondary degree-granting institutions, by control and level of institution: Fall 1995 through fall 2020

[In thousands]

\begin{tabular}{|c|c|c|c|c|c|}
\hline \multirow[b]{2}{*}{ Year } & \multirow[b]{2}{*}{ Total } & \multicolumn{2}{|c|}{ Public } & \multicolumn{2}{|c|}{ Private } \\
\hline & & 4-year & 2-year & 4-year & 2-year \\
\hline \multicolumn{6}{|c|}{ Actual } \\
\hline 1995 & 10,337 & 4,757 & 2,994 & 2,418 & 168 \\
\hline 1996 & 10,482 & 4,767 & 3,028 & 2,467 & 219 \\
\hline 1997 & 10,615 & 4,814 & 3,056 & 2,525 & 220 \\
\hline 1998 & 10,699 & 4,869 & 3,011 & 2,599 & 220 \\
\hline 1999 & 10,944 & 4,945 & 3,075 & 2,694 & 229 \\
\hline 2000 & 11,267 & 5,026 & 3,241 & 2,770 & 231 \\
\hline 2001 & 11,766 & 5,194 & 3,445 & 2,894 & 233 \\
\hline 2002 & 12,331 & 5,406 & 3,655 & 3,033 & 237 \\
\hline 2003 & 12,689 & 5,558 & 3,684 & 3,186 & 260 \\
\hline 2004 & 13,001 & 5,641 & 3,707 & 3,377 & 276 \\
\hline 2005 & 13,201 & 5,728 & 3,662 & 3,533 & 277 \\
\hline 2006 & 13,403 & 5,825 & 3,679 & 3,631 & 268 \\
\hline 2007 & 13,783 & 5,994 & 3,745 & 3,775 & 268 \\
\hline 2008 & 14,394 & 6,140 & 3,922 & 4,030 & 302 \\
\hline 2009 & 15,496 & 6,452 & 4,298 & 4,357 & 389 \\
\hline \multicolumn{6}{|c|}{ Projected } \\
\hline 2010 & 15,593 & 6,501 & 4,312 & 4,392 & 388 \\
\hline 2011 & 15,677 & 6,541 & 4,326 & 4,421 & 389 \\
\hline 2012 & 15,692 & 6,548 & 4,325 & 4,430 & 389 \\
\hline 2013 & 15,840 & 6,608 & 4,364 & 4,476 & 392 \\
\hline 2014 & 16,099 & 6,711 & 4,437 & 4,553 & 398 \\
\hline 2015 & 16,329 & 6,802 & 4,502 & 4,621 & 404 \\
\hline 2016 & 16,548 & 6,888 & 4,565 & 4,686 & 409 \\
\hline 2017 & 16,745 & 6,963 & 4,626 & 4,741 & 415 \\
\hline 2018 & 16,954 & 7,043 & 4,692 & 4,798 & 421 \\
\hline 2019 & 17,159 & 7,124 & 4,756 & 4,852 & 427 \\
\hline 2020 & 17,307 & 7,183 & 4,804 & 4,889 & 432 \\
\hline
\end{tabular}

NOTE: Detail may not sum to totals because of rounding. Some data have been revised from previously published figures. Mean absolute percentage errors of selected education statistics can be found in table A-2, appendix A.

SOURCE: U.S. Department of Education, National Center for Education Statistics, Integrated Postsecondary Education Data System, "Fall Enrollment Survey" (IPEDS-EF:95-99), and Spring 2001 through Spring 2010; and Enrollment in Degree-Granting Institutions Model, 1980-2009. (This table was prepared February 2011.) 
Table 32. Actual and projected numbers for associate's degrees conferred by postsecondary degree-granting institutions, by sex of recipient: 1995-96 through 2020-21

\begin{tabular}{|c|c|c|c|}
\hline Year & Total & Men & Women \\
\hline \multicolumn{4}{|l|}{ Actual } \\
\hline 1995-96 & 555,216 & 219,514 & 335,702 \\
\hline 1996-97 & 571,226 & 223,948 & 347,278 \\
\hline $1997-98$ & 558,555 & 217,613 & 340,942 \\
\hline 1998-99 & 559,954 & 218,417 & 341,537 \\
\hline 1999-2000 & 564,933 & 224,721 & 340,212 \\
\hline 2000-01 & 578,865 & 231,645 & 347,220 \\
\hline 2001-02 & 595,133 & 238,109 & 357,024 \\
\hline 2002-03 & 634,016 & 253,451 & 380,565 \\
\hline 2003-04 & 665,301 & 260,033 & 405,268 \\
\hline 2004-05 & 696,660 & 267,536 & 429,124 \\
\hline 2005-06 & 713,066 & 270,095 & 442,971 \\
\hline 2006-07 & 728,114 & 275,187 & 452,927 \\
\hline 2007-08 & 750,164 & 282,521 & 467,643 \\
\hline 2008-09 & 787,325 & 298,141 & 489,184 \\
\hline \multicolumn{4}{|l|}{ Projected } \\
\hline 2009-10 & 835,000 & 319,000 & 516,000 \\
\hline $2010-11$ & 863,000 & 324,000 & 539,000 \\
\hline $2011-12$ & 895,000 & 337,000 & 558,000 \\
\hline $2012-13$ & 899,000 & 338,000 & 561,000 \\
\hline $2013-14$ & 906,000 & 341,000 & 565,000 \\
\hline 2014-15 & 915,000 & 343,000 & 571,000 \\
\hline $2015-16$ & 927,000 & 347,000 & 580,000 \\
\hline $2016-17$ & 940,000 & 350,000 & 590,000 \\
\hline 2017-18 & 953,000 & 354,000 & 599,000 \\
\hline 2018-19 & 967,000 & 358,000 & 609,000 \\
\hline 2019-20 & 981,000 & 362,000 & 619,000 \\
\hline $2020-21$ & 994,000 & 366,000 & 628,000 \\
\hline
\end{tabular}

NOTE: Some data have been revised from previously published figures. Detail may not sum to totals because of rounding. Mean absolute percentage errors of selected education statistics can be found in table A-2, appendix A.

SOURCE: U.S. Department of Education, National Center for Education Statistics, Integrated Postsecondary Education Data System, "Completions Survey" (IPEDS-C:96-99), and Fall 2000 through Fall 2009; and Degrees Conferred Model, 1975-76 through 2008-09. (This table was prepared March 2011.) 
Table 33. Actual and projected numbers for bachelor's degrees conferred by postsecondary degree-granting institutions, by sex of recipient: 1995-96 through 2020-21

\begin{tabular}{|c|c|c|c|}
\hline Year & Total & Men & Women \\
\hline \multicolumn{4}{|l|}{ Actual } \\
\hline 1995-96 & $1,164,792$ & 522,454 & 642,338 \\
\hline 1996-97 & $1,172,879$ & 520,515 & 652,364 \\
\hline 1997-98 & $1,184,406$ & 519,956 & 664,450 \\
\hline 1998-99 & $1,200,303$ & 518,746 & 681,557 \\
\hline 1999-2000 & $1,237,875$ & 530,367 & 707,508 \\
\hline 2000-01 & $1,244,171$ & 531,840 & 712,331 \\
\hline 2001-02 & $1,291,900$ & 549,816 & 742,084 \\
\hline 2002-03 & $1,348,811$ & 573,258 & 775,553 \\
\hline 2003-04 & $1,399,542$ & 595,425 & 804,117 \\
\hline 2004-05 & $1,439,264$ & 613,000 & 826,264 \\
\hline 2005-06 & $1,485,242$ & 630,600 & 854,642 \\
\hline 2006-07 & $1,524,092$ & 649,570 & 874,522 \\
\hline 2007-08 & $1,563,069$ & 667,928 & 895,141 \\
\hline 2008-09 & $1,601,368$ & 685,382 & 915,986 \\
\hline \multicolumn{4}{|l|}{ Projected } \\
\hline 2009-10 & $1,673,000$ & 717,000 & 956,000 \\
\hline 2010-11 & $1,715,000$ & 735,000 & 980,000 \\
\hline 2011-12 & $1,781,000$ & 765,000 & $1,016,000$ \\
\hline 2012-13 & $1,791,000$ & 766,000 & $1,025,000$ \\
\hline 2013-14 & $1,805,000$ & 769,000 & $1,036,000$ \\
\hline 2014-15 & $1,817,000$ & 771,000 & $1,046,000$ \\
\hline 2015-16 & $1,835,000$ & 775,000 & $1,060,000$ \\
\hline 2016-17 & $1,858,000$ & 782,000 & $1,076,000$ \\
\hline 2017-18 & $1,879,000$ & 787,000 & $1,092,000$ \\
\hline 2018-19 & $1,901,000$ & 794,000 & $1,107,000$ \\
\hline 2019-20 & $1,923,000$ & 801,000 & $1,123,000$ \\
\hline 2020-21 & $1,945,000$ & 808,000 & $1,137,000$ \\
\hline
\end{tabular}

NOTE: Some data have been revised from previously published figures. Detail may not sum to totals because of rounding. Mean absolute percentage errors of selected education statistics can be found in table A-2, appendix A.

SOURCE: U.S. Department of Education, National Center for Education Statistics, Integrated Postsecondary Education Data System, "Completions Survey" (IPEDS-C:96-99), and Fall 2000 through Fall 2009; and Degrees Conferred Model, 1975-76 through 2008-09. (This table was prepared March 2011.) 
Table 34. Actual and projected numbers for master's degrees conferred by postsecondary degree-granting institutions, by sex of recipient: 1995-96 through 2020-21

\begin{tabular}{|c|c|c|c|}
\hline Year & Total & Men & Women \\
\hline \multicolumn{4}{|l|}{ Actual } \\
\hline 1995-96 & 406,301 & 179,081 & 227,220 \\
\hline 1996-97 & 419,401 & 180,947 & 238,454 \\
\hline 1997-98 & 430,164 & 184,375 & 245,789 \\
\hline 1998-99 & 439,986 & 186,148 & 253,838 \\
\hline 1999-2000 & 457,056 & 191,792 & 265,264 \\
\hline 2000-01 & 468,476 & 194,351 & 274,125 \\
\hline 2001-02 & 482,118 & 199,120 & 282,998 \\
\hline 2002-03 & 513,339 & 211,664 & 301,675 \\
\hline 2003-04 & 558,940 & 229,545 & 329,395 \\
\hline 2004-05 & 574,618 & 233,590 & 341,028 \\
\hline 2005-06 & 594,065 & 237,896 & 356,169 \\
\hline 2006-07 & 604,607 & 238,189 & 366,418 \\
\hline 2007-08 & 625,023 & 246,491 & 378,532 \\
\hline 2008-09 & 656,784 & 259,998 & 396,786 \\
\hline \multicolumn{4}{|l|}{ Projected } \\
\hline 2009-10 & 691,000 & 276,000 & 415,000 \\
\hline $2010-11$ & 712,000 & 291,000 & 421,000 \\
\hline $2011-12$ & 730,000 & 299,000 & 430,000 \\
\hline $2012-13$ & 741,000 & 302,000 & 439,000 \\
\hline 2013-14 & 756,000 & 306,000 & 450,000 \\
\hline 2014-15 & 776,000 & 312,000 & 463,000 \\
\hline 2015-16 & 795,000 & 319,000 & 476,000 \\
\hline $2016-17$ & 813,000 & 325,000 & 488,000 \\
\hline 2017-18 & 829,000 & 330,000 & 499,000 \\
\hline 2018-19 & 844,000 & 335,000 & 510,000 \\
\hline 2019-20 & 856,000 & 337,000 & 519,000 \\
\hline $2020-21$ & 865,000 & 338,000 & 527,000 \\
\hline
\end{tabular}

NOTE: Some data have been revised from previously published figures. Detail may not sum to totals because of rounding. Mean absolute percentage errors of selected education statistics can be found in table A-2, appendix A.

SOURCE: U.S. Department of Education, National Center for Education Statistics, Integrated Postsecondary Education Data System, "Completions Survey" (IPEDS-C:96-99), and Fall 2000 through Fall 2009; and Degrees Conferred Model, 1975-76 through 2008-09. (This table was prepared March 2011.) 
Table 35. Actual and projected numbers for doctor's degrees conferred by postsecondary degree-granting institutions, by sex of recipient: 1995-96 through 2020-21

\begin{tabular}{|c|c|c|c|}
\hline Year & Total & Men & Women \\
\hline \multicolumn{4}{|l|}{ Actual } \\
\hline 1995-96 & 44,652 & 26,841 & 17,811 \\
\hline 1996-97 & 45,876 & 27,146 & 18,730 \\
\hline 1997-98 & 46,010 & 26,664 & 19,346 \\
\hline 1998-99 & 44,077 & 25,146 & 18,931 \\
\hline 1999-2000 & 44,808 & 25,028 & 19,780 \\
\hline 2000-01 & 44,904 & 24,728 & 20,176 \\
\hline 2001-02 & 44,160 & 23,708 & 20,452 \\
\hline 2002-03 & 46,042 & 24,351 & 21,691 \\
\hline 2003-04 & 48,378 & 25,323 & 23,055 \\
\hline 2004-05 & 52,631 & 26,973 & 25,658 \\
\hline 2005-06 & 56,067 & 28,634 & 27,433 \\
\hline 2006-07 & 60,616 & 30,251 & 30,365 \\
\hline 2007-08 & 63,712 & 31,215 & 32,497 \\
\hline 2008-09 & 67,716 & 32,279 & 35,437 \\
\hline \multicolumn{4}{|l|}{ Projected } \\
\hline $2009-10$ & 70,400 & 33,400 & 37,000 \\
\hline $2010-11$ & 73,700 & 34,600 & 39,100 \\
\hline 2011-12 & 76,900 & 35,700 & 41,200 \\
\hline 2012-13 & 80,200 & 36,800 & 43,300 \\
\hline 2013-14 & 83,400 & 38,000 & 45,400 \\
\hline 2014-15 & 86,600 & 39,100 & 47,600 \\
\hline 2015-16 & 89,900 & 40,200 & 49,700 \\
\hline $2016-17$ & 93,100 & 41,400 & 51,800 \\
\hline 2017-18 & 96,400 & 42,500 & 53,900 \\
\hline 2018-19 & 99,600 & 43,600 & 56,000 \\
\hline 2019-20 & 102,800 & 44,700 & 58,100 \\
\hline 2020-21 & 106,100 & 45,900 & 60,200 \\
\hline
\end{tabular}

NOTE: Doctor's degrees include Ph.D., Ed.D., and comparable degrees at the doctoral level. See Glossary. Excluded are first-professional degrees, such as M.D., D.D.S., and law degrees. Some data have been revised from previously published figures. Detail may not sum to totals because of rounding. Mean absolute percentage errors of selected education statistics can be found in table A-2, appendix A.

SOURCE: U.S. Department of Education, National Center for Education Statistics, Integrated Postsecondary Education Data System, "Completions Survey" (IPEDS-C:96-99), and Fall 2000 through Fall 2009; and Degrees Conferred Model, 1975-76 through 2008-09. (This table was prepared March 2011.) 
Table 36. Actual and projected numbers for first-professional degrees conferred by postsecondary degree-granting institutions, by sex of recipient: 1995-96 through 2020-21

\begin{tabular}{|c|c|c|c|}
\hline Year & Total & Men & Women \\
\hline \multicolumn{4}{|l|}{ Actual } \\
\hline 1995-96 & 76,734 & 44,748 & 31,986 \\
\hline 1996-97 & 78,730 & 45,564 & 33,166 \\
\hline 1997-98 & 78,598 & 44,911 & 33,687 \\
\hline 1998-99 & 78,439 & 44,339 & 34,100 \\
\hline 1999-2000 & 80,057 & 44,239 & 35,818 \\
\hline 2000-01 & 79,707 & 42,862 & 36,845 \\
\hline 2001-02 & 80,698 & 42,507 & 38,191 \\
\hline 2002-03 & 80,897 & 41,887 & 39,010 \\
\hline 2003-04 & 83,041 & 42,169 & 40,872 \\
\hline 2004-05 & 87,289 & 43,849 & 43,440 \\
\hline 2005-06 & 87,655 & 44,038 & 43,617 \\
\hline 2006-07 & 90,064 & 45,057 & 45,007 \\
\hline 2007-08 & 91,309 & 45,916 & 45,393 \\
\hline 2008-09 & 92,004 & 46,900 & 45,104 \\
\hline \multicolumn{4}{|l|}{ Projected } \\
\hline 2009-10 & 95,100 & 48,400 & 46,700 \\
\hline $2010-11$ & 99,800 & 50,600 & 49,300 \\
\hline $2011-12$ & 103,200 & 52,500 & 50,700 \\
\hline $2012-13$ & 104,000 & 53,400 & 50,600 \\
\hline 2013-14 & 105,100 & 53,900 & 51,200 \\
\hline 2014-15 & 106,700 & 54,600 & 52,100 \\
\hline 2015-16 & 109,100 & 55,500 & 53,600 \\
\hline $2016-17$ & 111,800 & 56,500 & 55,300 \\
\hline 2017-18 & 114,200 & 57,500 & 56,800 \\
\hline 2018-19 & 116,300 & 58,200 & 58,100 \\
\hline 2019-20 & 118,000 & 58,900 & 59,100 \\
\hline 2020-21 & 119,200 & 59,300 & 59,900 \\
\hline
\end{tabular}

NOTE: Some data have been revised from previously published figures. Detail may not sum to totals because of rounding. Mean absolute percentage errors of selected education statistics can be found in table A-2, appendix A.

SOURCE: U.S. Department of Education, National Center for Education Statistics, Integrated Postsecondary Education Data System, "Completions Survey" (IPEDS-C:96-99), and Fall 2000 through Fall 2009; and Degrees Conferred Model, 1975-76 through 2008-09. (This table was prepared March 2011.) 


\section{Technical Appendixes}




\section{Appendix A \\ Introduction to Projection Methodology}

\section{A.0. INTRODUCTION TO PROJECTION METHODOLOGY}

\section{Content of appendix A}

Since its inception in 1964, the Projections of Education Statistics series has been providing projections of key education statistics to policy makers, educators, researchers, the press, and the general public. This edition of Projections of Education Statistics is the thirty-ninth in the series.

Appendix A contains this introduction, which provides a general overview of the projection methodology, as well as six additional sections, which discuss the specific methodology for the different statistics projected:

»A.0. Introduction to Projection Methodology;

»A.1. Elementary and Secondary Enrollment;

»A.2. High School Graduates;

»A.3. Elementary and Secondary Teachers;

»A.4. Expenditures for Public Elementary and Secondary Education;

»A.5. Enrollment in Postsecondary Degree-Granting Institutions; and

» A.6. Postsecondary Degrees Conferred.

This introduction

» outlines the two major techniques used to make the projections;

» summarizes key demographic and economic assumptions underlying the projections;

》) examines the accuracy of the projections; and

1) introduces the subsequent sections of appendix A.

\section{Projection techniques}

Two major projection techniques were used to develop the projections presented in this publication:

» Exponential smoothing was the technique used in the projections of elementary and secondary enrollments and high school graduates. This technique also played a role in the projections of teachers at the elementary and secondary level, as well as enrollments and degrees conferred at the postsecondary level.

» Multiple linear regression was the primary technique used in the projections of teachers and expenditures at the elementary and secondary level, as well as enrollments and degrees conferred at the postsecondary level.

\section{Exponential smoothing}

Two different types of exponential smoothing, single exponential smoothing and double exponential smoothing, were used in producing the projections presented in this publication. 
Single exponential smoothing was used when the historical data had a basically horizontal pattern. Single exponential smoothing produces a single forecast for all years in the forecast period. In developing projections of elementary and secondary enrollments, for example, the rate at which students progress from one particular grade to the next (e.g., from grade 2 to grade 3) was projected using single exponential smoothing. Thus, this percentage was assumed to be constant over the forecast period.

In general, exponential smoothing places more weight on recent observations than on earlier ones. The weights for observations decrease exponentially as one moves further into the past. As a result, the older data have less influence on the projections. The rate at which the weights of older observations decrease is determined by the smoothing constant.

When using single exponential smoothing for a time series, $P_{t}$, a smoothed series, $\hat{P}$, is computed recursively by evaluating

$$
\hat{P}_{t}=\propto P_{t}+(1-\propto) \hat{P}_{t-1}
$$

where $0<\propto \leq 1$ is the smoothing constant.

By repeated substitution, we can rewrite the equation as

$$
\hat{P}_{t}=\propto \sum_{s=0}^{t-1}(1-\propto)^{s} P_{t-s}
$$

where time, $s$, goes from the first period in the time series, 0 , to time period $t-1$.

The forecasts are constant for all years in the forecast period. The constant equals

$$
\hat{P}_{T+k}=\hat{P}_{T}
$$

where $T$ is the last year in the estimation sample and $\mathrm{k}>0$.

These equations illustrate that the projection is a weighted average based on exponentially decreasing weights. For higher smoothing constants, weights for earlier observations decrease more rapidly than for lower smoothing constants.

For each of the approximately 1,200 single exponential smoothing equations in this edition of Projections of Education Statistics, a smoothing constant was individually chosen to minimize the sum of squared forecast errors for that equation. The smoothing constants used to produce the projections in this report ranged from 0.001 to 0.999 .

Double exponential smoothing is an extension of single exponential smoothing that allows the forecasting of data with trends. It produces different forecasts for different years in the forecast period. Double exponential smoothing with two smoothing constants was used to forecast the number of doctor's degrees awarded to men and women.

The smoothing forecast using double exponential smoothing is found using the three equations:

$$
\begin{aligned}
& \hat{P}_{t+k}=a_{t}+b_{t} k \\
& a_{t}=\propto P_{t}+(1-\propto)\left(a_{t-1}+b_{t-1}\right) \\
& b_{t}=\beta\left(a_{t}-a_{t-1}\right)+(1-\beta) b_{t-1}
\end{aligned}
$$

where $a_{t}$ denotes an estimate of the level of the series at time $t, b_{t}$ denotes an estimate of the level of the series at time $t$, and 0 $<\propto, \beta<1$ are the smoothing constants.

Forecasts from double smoothing are computed as

$$
\hat{P}_{T+k}=a_{T}+b_{T} k
$$

where $T$ is the last year in the estimation sample and $\mathrm{k}>0$. The last expression shows that forecasts from double smoothing lie on a linear trend with intercept $a_{T}$ and slope $b_{T}$. Single exponential smoothing can be viewed as a special case of double exponential smoothing where the impact that time has on the forecasts has been eliminated (i.e., requiring the slope term $b_{t}$ to equal 0.0 ). 
The smoothing constants for each of the two double exponential smoothing equations used for this report were selected using a search algorithm that finds the pair of smoothing constants that together minimizes the sum of forecast errors for their equation.

There were two differences in the exponential smoothing methodology used for this edition of Projections of Education Statistics compared to that used in previous editions. In previous editions of Projections of Education Statistics, a smoothing constant of 0.4 was used for both single and double exponential smoothing. This change was made because there is evidence that it is preferable to select smoothing constants by using a selection criterion such as minimizing the sum of squared forecast errors rather than by using a set value for the smoothing constant. The minimization of the sum of squared forecast errors was used because it is the most commonly used selection criterion. The second difference is that this edition of Projections of Education Statistics uses two smoothing constants for double exponential smoothing, while previous editions used only one smoothing constant. This second change was made to eliminate the assumption that the two smoothing constants were equal. There was no systematic attempt to measure the impact of these two changes in methodology compared to the methods used in previous editions of Projections of Education Statistics. For more information about exponential smoothing, including the choice of the smoothing constants, see Gardner (1985) and Makridakis, Wheelwright, and Hyndman (1998).

\section{Multiple linear regression}

Multiple linear regression was used in cases where a strong relationship exists between the variable being projected (the dependent variable) and independent variables. This technique can be used only when accurate data and reliable projections of the independent variables are available. Key independent variables for this publication include demographic and economic factors. For example, current expenditures for public elementary and secondary education are related to economic factors such as disposable income and education revenues from state sources. The sources of the demographic and economic projections used for this publication are discussed below, under "Assumptions."

The equations in this appendix should be viewed as forecasting rather than structural equations. That is, the equations are intended only to project values for the dependent variables, not to reflect all elements of underlying social, political, and economic structures. Lack of available data precluded the building of large-scale structural models. The particular equations shown were selected on the basis of their statistical properties, such as coefficients of determination $\left(R^{2} s\right)$, the $t$-statistics of the coefficients, the Durbin-Watson statistic, the Breusch-Godfrey Serial Correlation LM test statistic, and residual plots.

The functional form primarily used is the multiplicative model. When used with two independent variables, this model takes the form:

$$
Y=a \cdot X_{1}^{b_{1}} \cdot X_{2}^{b_{2}}
$$

This equation can easily be transformed into the linear form by taking the natural $\log (\ln )$ of both sides of the equation:

$$
\ln (Y)=\ln (a)+b_{1} \ln X_{1}+b_{2} \ln X_{2}
$$

One property of this model is that the coefficient of an independent variable shows how responsive in percentage terms the dependent variable is to a one percent change in that independent variable (also called the elasticity). For example, a 1 percent change in $X_{1}$ in the above equation would lead to a $b_{1}$ percent change in $Y$.

\section{Assumptions}

All projections are based on underlying assumptions, and these assumptions determine projection results to a large extent. It is important that users of projections understand the assumptions to determine the acceptability of projected time series for their purposes. All the projections in this publication are to some extent dependent on demographic and/or economic assumptions.

\section{Demographic assumptions}

Many of the projections in this publication are demographically based on the U.S. Census Bureau's 2008 National Population Projections (August 2008) and the State Interim Population Projections (April 2005).

The two sets of Census Bureau population projections are produced using cohort-component models. In order for the national-level population projections by age, sex, and race/ethnicity to be consistent with the most recent historical estimates released by the Census Bureau, the projections were ratio-adjusted by applying the ratio of the last historical estimate to the corresponding projections year to the projections for each age, sex, and race/ethnicity combination. This allows for a consistent set of historical estimates and projections. For more information on the methodology used for Census Bureau population projections, see appendix C, Data Sources. 
The enrollment projections in this publication depend on Census Bureau population projections for the various age groups that attend school. The future fertility rate assumption (along with corresponding projections of female populations)

determines projections of the number of births, a key factor for population projections. The fertility rate assumption plays a major role in determining population projections for the age groups enrolled in nursery school, kindergarten, and elementary grades. The effects of the fertility rate assumption are more pronounced toward the end of the forecast period, while immigration assumptions affect all years. For enrollments in secondary grades and college, the fertility rate assumption is of no consequence, since all the population cohorts for these enrollment ranges have already been born.

\section{Economic assumptions}

Various economic variables are used in the forecasting models for numbers of elementary and secondary teachers, public elementary and secondary school expenditures, and postsecondary enrollment.

The source of these variables is the trend scenario of the "U.S. Monthly Model November 2010: Short-Term Projections" developed by the economic consulting firm IHS Global Insight. The trend scenario depicts a mean of possible paths that the economy could take over the forecast period, barring major shocks. The economy, in this scenario, evolves smoothly, without major fluctuations.

\section{More information about specific assumptions}

For details about the primary assumptions used in this edition of Projections of Education Statistics, see table A-1 on page 82.

\section{Accuracy of the projections}

Projections of time series usually differ from the final reported data due to errors from many sources. This is because of the inherent nature of the statistical universe from which the basic data are obtained and the properties of projection methodologies, which depend on the validity of many assumptions.

The mean absolute percentage error (MAPE) is one way to express the forecast accuracy of past projections. This measure expresses the average absolute value of errors over past projections in percentage terms. For example, an analysis of projection errors over the past 27 editions of Projections of Education Statistics indicates that the MAPEs for public school enrollment in grades PK-12 for lead times of 1, 2, 5, and 10 years were $0.3,0.6,1.3$, and 2.4 percent, respectively. For the 1-year-out projection, this means that one would expect the projection to be within 0.3 percent of the actual value, on average.

For a list of MAPEs for selected national statistics in this publication, see table A-2 on page 83. Sections A.1 through A.5 each contains at least one text table (tables A through F) that presents the MAPEs for the key national statistics of that section. Each text table appears directly after the discussion of accuracy of that section's national projections. For a list of MAPEs by state and region for public elementary and secondary enrollment, see tables A-7 through A-9 on pages 92-97 and for a list of MAPEs by state and region for the number of high school graduates in public schools, see table A-10 on pages 102-103.

Tables A-3 and A-4 present an example of how the MAPEs were constructed using actual values for national public elementary and secondary enrollment projections for schools years 2005 through 2008 and enrollment projections from the last four editions of Projections of Education Statistics. The top panel of table A-3 shows the actual values for school years 2005 through 2008 and enrollment projections for each year from Projections of Education Statistics to 2016 with the number of projections decreasing by one for each subsequent edition. The bottom panel of table A-3 shows the percentage differences between the actual values and the projected values. For example, the projected value for 2005 presented in Projections of Education Statistics to 2016 was 0.2 lower than the actual value for that year.

The top panel of table A- 4 shows the absolute value of the percent differences from table A-3 arranged by lead time rather than year. Hence, the 0.3 appearing in the column for lead times of 1 year and the row for projections from Projections of Education Statistics to 2017 indicates that projection of the 1-year-out forecast from Projections of Education Statistics to 2017 differed by 0.3 in absolute terms from its actual value. The MAPEs for each lead time shown in the bottom panel of table A-4 were calculated by computing the average of the absolute values of the percentage differences for that lead time. These MAPEs are different from the MAPEs for public elementary and secondary enrollment projections elsewhere in this report because the MAPEs in the example were calculated using only the last 4 editions of Projections of Education Statistics.

The number of years used in the analysis of the projection error differs by statistics both because projections of additional education statistics have been added to the report over time and because, for some statistics, there have been such a substantial change in the methodology used to produce the projections that the projections produced using the earlier methodology were not included in the analysis of the projection error. MAPEs are presented for a statistic only after it has been produced using substantially the same methodology in five previous editions of Projections of Education Statistics. 


\section{Demographic assumptions}

Population

18- to 24-year-old population

25- to 29-year-old population

30- to 34-year-old population

35- to 44-year-old population

\section{Economic assumptions}

Disposable income per capita in constant dollars

Education revenue receipts from state sources per capita in constant dollars

Inflation rate

Unemployment rate (men)

Ages 18 and 19

Ages 20 to 24

Age 25 and over

\section{Unemployment rate (women)}

Ages 18 and 19

Ages 20 to 24

Age 25 and over
Projections are consistent with the Census Bureau estimates ${ }^{1}$ Census Bureau projection: average annual growth rate of $0.1 \%$ Census Bureau projection: average annual growth rate of $0.7 \%$ Census Bureau projection: average annual growth rate of $1.4 \%$ Census Bureau projection: average annual growth rate of $0.4 \%$

Annual percent changes range between $0.1 \%$ and $2.6 \%$ with an annual growth rate of $1.3 \%$ Annual percent changes range between $-1.6 \%$ and $2.7 \%$ with an annual growth rate of $1.2 \%$ Inflation rate ranges between $1.0 \%$ and $2.2 \%$

Remains between $17.4 \%$ and $28.4 \%$ Remains between $10.3 \%$ and $17.8 \%$ Remains between $4.6 \%$ and $8.4 \%$

Remains between $13.1 \%$ and $20.4 \%$ Remains between $8.1 \%$ and $13.1 \%$ Remains between $4.3 \%$ and $7.0 \%$

${ }^{1}$ As the Census Bureau projections were not updated to reflect 2010 Census Bureau population estimates, the Census Bureau age-specific population projections for each year were adjusted by multiplying the ratio of the total Census Bureau estimate for 2010 to the total Census Bureau projection for 2010.

SOURCE: U.S. Department of Commerce, Census Bureau, Population Estimates, retrieved October 4, 2010, from http://www.census.gov/popest/ national/; and Population Projections, retrieved November 2, 2008, from http://www.census.gov/population/www/projections/

2008projections.html; and IHS Global Insight, "U.S. Monthly Model November 2010 Short-Term Projections." (This table was prepared March 2011.) 
Table A-2. Mean absolute percentage errors (MAPEs) by lead time for selected statistics in all public elementary and secondary schools and postsecondary degree-granting institutions: 2011

\begin{tabular}{|c|c|c|c|c|c|c|c|c|c|c|}
\hline \multirow[b]{2}{*}{ Statistic } & \multicolumn{10}{|c|}{ Lead time (years) } \\
\hline & 1 & 2 & 3 & 4 & 5 & 6 & 7 & 8 & 9 & 10 \\
\hline \multicolumn{11}{|l|}{ Public elementary and secondary schools } \\
\hline Prekindergarten-12 enrollment ${ }^{1}$ & 0.3 & 0.6 & 0.8 & 1.1 & 1.3 & 1.5 & 1.7 & 2.0 & 2.2 & 2.4 \\
\hline Prekindergarten-8 enrollment ${ }^{1}$ & 0.4 & 0.7 & 1.0 & 1.2 & 1.3 & 1.7 & 2.0 & 2.5 & 2.8 & 3.2 \\
\hline $9-12$ enrollment $^{1}$ & 0.3 & 0.6 & 0.9 & 1.1 & 1.3 & 1.5 & 1.9 & 2.3 & 2.4 & 2.3 \\
\hline High school graduates ${ }^{2}$ & 1.0 & 1.0 & 1.5 & 1.7 & 1.6 & 2.1 & 2.8 & 3.7 & 4.2 & 4.0 \\
\hline Elementary and secondary teachers ${ }^{3}$ & 1.0 & 1.4 & 1.7 & 2.3 & 2.9 & 3.4 & 4.1 & 4.4 & 5.1 & 5.9 \\
\hline Total current expenditures ${ }^{4}$ & 1.3 & 2.0 & 2.1 & 2.2 & 2.6 & 3.3 & 4.1 & 4.3 & 4.0 & 4.1 \\
\hline Current expenditures per pupil in fall enrollment ${ }^{4}$ & 1.3 & 2.0 & 2.0 & 2.1 & 3.0 & 3.6 & 4.4 & 4.9 & 5.2 & 5.5 \\
\hline \multicolumn{11}{|l|}{ Private elementary and secondary schools ${ }^{5}$} \\
\hline Prekindergarten-12 enrollment & 3.4 & 4.6 & 3.7 & 7.2 & 7.7 & 10.6 & 9.3 & 9.4 & 8.1 & 6.3 \\
\hline Prekindergarten-8 enrollment & 3.5 & 4.9 & 4.1 & 8.0 & 9.2 & 12.1 & 10.6 & 10.4 & 10.2 & 7.9 \\
\hline 9-12 enrollment & 3.0 & 3.8 & 2.3 & 4.3 & 2.8 & 5.8 & 5.7 & 6.1 & 1.3 & 1.3 \\
\hline High school graduates & 0.9 & 0.9 & 1.6 & 2.8 & 5.0 & 6.2 & 4.9 & 4.8 & 1.6 & 1.6 \\
\hline \multicolumn{11}{|l|}{ Postsecondary degree-granting institutions ${ }^{6}$} \\
\hline Total enrollment & 1.6 & 2.8 & 3.5 & 4.0 & 5.2 & 6.1 & 8.1 & 9.8 & 11.7 & 11.4 \\
\hline Men & 1.6 & 3.2 & 4.0 & 4.8 & 5.9 & 6.6 & 8.5 & 9.8 & 11.7 & 11.7 \\
\hline Women & 1.8 & 2.8 & 3.6 & 3.7 & 4.6 & 5.8 & 7.8 & 9.8 & 11.7 & 11.2 \\
\hline 4-year institutions & 1.7 & 2.9 & 3.9 & 4.7 & 5.9 & 6.8 & 9.0 & 10.9 & 13.1 & 13.2 \\
\hline 2-year institutions & 2.2 & 3.7 & 4.3 & 4.4 & 5.1 & 5.0 & 6.4 & 7.8 & 9.4 & 8.3 \\
\hline White & 0.4 & 1.1 & 2.3 & 2.8 & 3.6 & - & - & - & - & - \\
\hline Black or African American & 2.2 & 5.3 & 7.5 & 9.2 & 9.4 & - & - & - & - & - \\
\hline Hispanic or Latino & 2.0 & 4.7 & 7.0 & 9.0 & 9.5 & - & - & - & - & - \\
\hline Asian/Hawaiian or other Pacific Islander & 2.2 & 5.2 & 7.1 & 8.3 & 9.2 & - & - & - & - & - \\
\hline American Indian/Alaska Native & 4.4 & 6.4 & 3.9 & 4.3 & 4.6 & - & - & - & - & - \\
\hline Nonresident alien & 2.6 & 6.2 & 8.8 & 6.5 & 2.9 & - & - & - & - & - \\
\hline
\end{tabular}

- Not available.

${ }^{1}$ MAPEs for public prekindergarten-12 enrollments were calculated using the last 27 editions of Projections of Education Statistics.

2 MAPEs for public high school graduates were calculated from the past 20 editions of Projections of Education Statistics.

${ }^{3}$ Data for teachers expressed in full-time equivalents. MAPEs for teachers were calculated from the past 20 editions containing teacher projections.

${ }^{4}$ In constant dollars based on the Consumer Price Index for all urban consumers, Bureau of Labor Statistics, U.S. Department of Labor. MAPEs for current expenditures were calculated using projections from the last 20 editions containing current expenditure projections.

${ }^{5}$ MAPEs for private prekindergarten-12 enrollments and high school graduates were calculated from the past 9 editions.

${ }^{6}$ MAPEs for postsecondary degree-granting institution enrollments were calculated using the last 12 editions of Projections of Education Statistics. NOTE: Mean absolute percentage error is the average value over past projections of the absolute values of errors expressed in percentage terms. No MAPEs are presented for degrees conferred as the current models used for producing these projections have only been used for two other editions of Projections of Education Statistics. Calculations were made using unrounded numbers. Some data have been revised from previously published figures.

SOURCE: U.S. Department of Education, National Center for Education Statistics, Projections of Education Statistics, various issues. (This table was prepared February 2011.) 
Table A-3. Example of constructing mean absolute percentage errors, part 1

\begin{tabular}{|c|c|c|c|c|}
\hline \multirow[b]{2}{*}{ Source } & \multicolumn{4}{|c|}{ Year of data } \\
\hline & 2005 & 2006 & 2007 & 2008 \\
\hline & \multicolumn{4}{|c|}{ Enrollment in thousands } \\
\hline \multirow[t]{2}{*}{ Actual } & 49,113 & 49,316 & 49,293 & 49,265 \\
\hline & \multicolumn{4}{|c|}{ Projected enrollment, in thousands } \\
\hline Projections of Education Statistics 2016 & 49,028 & 49,370 & 49,610 & 49,812 \\
\hline Projections of Education Statistics 2017 & $\dagger$ & 49,464 & 49,644 & 49,825 \\
\hline Projections of Education Statistics 2018 & $\dagger$ & $\dagger$ & 49,470 & 49,623 \\
\hline \multirow[t]{2}{*}{ Projections of Education Statistics 2019} & $\dagger$ & $\dagger$ & $\dagger$ & 49,265 \\
\hline & \multicolumn{4}{|c|}{ Percentage difference between actual and projected values } \\
\hline Projections of Education Statistics 2016 & -0.2 & 0.1 & 0.6 & 1.1 \\
\hline Projections of Education Statistics 2017 & $\dagger$ & 0.3 & 0.7 & 1.1 \\
\hline Projections of Education Statistics 2018 & $\dagger$ & $\dagger$ & 0.4 & 0.7 \\
\hline Projections of Education Statistics 2019 & $\dagger$ & $\dagger$ & $\dagger$ & \# \\
\hline
\end{tabular}

† Not applicable.

\# Rounds to zero.

SOURCE: U.S. Department of Education, National Center for Education Statistics, Common Core of Data (CCD), "State Nonfiscal Survey of Public Elementary/Secondary Education," 2005-06 through 2008-09; and Projections of Education Statistics, various editions. (This table was prepared January 2011.)

Table A-4. Example of constructing mean absolute percentage errors, part 2

\begin{tabular}{|c|c|c|c|c|}
\hline \multirow[b]{2}{*}{ Source } & \multicolumn{4}{|c|}{ Lead time (years) } \\
\hline & 1 & 2 & 3 & 4 \\
\hline & \multicolumn{4}{|c|}{ Absolute value of percentage difference between actual and projected values } \\
\hline Projections of Education Statistics 2016 & 0.2 & 0.1 & 0.6 & 1.1 \\
\hline Projections of Education Statistics 2017 & 0.3 & 0.7 & 1.1 & $\dagger$ \\
\hline Projections of Education Statistics 2018 & 0.4 & 0.7 & $\dagger$ & $\dagger$ \\
\hline \multirow[t]{2}{*}{ Projections of Education Statistics 2019} & $\#$ & $\dagger$ & $\dagger$ & $\dagger$ \\
\hline & \multicolumn{4}{|c|}{ Mean absolute percentage error } \\
\hline Example & 0.2 & 0.5 & 0.9 & 1.1 \\
\hline
\end{tabular}

† Not applicable.

\# Rounds to zero.

NOTE: The mean absolute percentage errors presented on this table are for illustrative purpose only.

SOURCE: U.S. Department of Education, National Center for Education Statistics, Common Core of Data (CCD), "State Nonfiscal Survey of Public Elementary/Secondary Education," 2005-06 through 2008-09; and Projections of Education Statistics, various editions. (This table was prepared January 2011.) 


\section{Projections in this edition}

This edition of Projections of Education Statistics presents projected trends in elementary and secondary enrollment from 2009 to 2020. These projections were made using three models:

» The National Elementary and Secondary Enrollment Model was used to project total, public, and private school enrollments for the nation by grade level and for ungraded elementary and ungraded secondary programs.

» The State Public Elementary and Secondary Enrollment Model was used to project total public school enrollments by grade level for individual states and regions.

» The National Public Elementary and Secondary Enrollment by Race/Ethnicity Model was used to project public school enrollments for the nation by race/ethnicity and grade level.

All three elementary and secondary enrollment models used the following same methods.

\section{Overview of approach}

Two methods were used in all the elementary and secondary enrollment models:

» The grade progression rate method was used to project enrollments in grades 2 through 12 . In this method, a rate of progression from each grade ( 1 through 11 ) to the next grade (2 through 12) was projected using single exponential smoothing. (For example, the rate of progression from grade 2 to grade 3 is the current year's grade 3 enrollment expressed as a percentage of the previous year's grade 2 enrollment.) To calculate enrollment for each year in the forecast period, the progression rate for each grade was applied to the previous year's enrollment in the previous grade.

» The enrollment rate method was used to project prekindergarten, kindergarten, and first-grade enrollments as well as elementary special and ungraded and secondary special and ungraded enrollments. For each of these enrollment categories, the enrollment rate for the last year of actual data was used as the projected enrollment rate. To calculate enrollment for each year in the forecast period, the enrollment rate for each category was applied to the projected population in the appropriate age group.

\section{Assumptions underlying these methods}

The grade progression and enrollment rate methods assume that past trends in factors affecting public and private elementary and secondary school enrollments will continue over the forecast period. This assumption implies that all factors influencing enrollments will display future patterns consistent with past patterns. This method implicitly includes the net effect of such factors as migration, dropouts, deaths, nonpromotion, and transfers between public and private schools.

For more details on the use of the grade progression and enrollment rate methods, see "Procedures and equations used in all three elementary and secondary enrollment models," below.

\section{Procedures and equations used in all three elementary and secondary enrollment models}

The notation and equations that follow describe the basic procedures used to project elementary and secondary enrollments in each of the three elementary and secondary enrollment models.

Let:

$i \quad$ Subscript denoting age

$j \quad=$ Subscript denoting grade

$t=$ Subscript denoting time

$T=$ Subscript of the first year in the forecast period

$N_{t}=$ Enrollment at the prekindergarten (nursery) level

$K_{\mathrm{t}}=$ Enrollment at the kindergarten level

$G_{j, t}=$ Enrollment in grade $j$

$E_{t}=$ Enrollment in elementary special and ungraded programs 
$S_{t}=$ Enrollment in secondary special and ungraded programs

$P_{i, t}=$ Population age $i$

$R_{j, t}=$ Progression rate for grade $j$

$R N_{t}=$ Enrollment rate for prekindergarten (nursery school)

$R K_{t}=$ Enrollment rate for kindergarten

$R G_{1, t}=$ Enrollment rate for grade 1

$R E_{t}=$ Enrollment rate for elementary special and ungraded programs

$R S_{t}=$ Enrollment rate for secondary special and ungraded programs.

Step 1. Calculate historical grade progression rates for each of grades 2 through 12. The first step in projecting the enrollments for grades 2 through 12 using the grade progression method was to calculate, for each grade, a progression rate for each year in the sample period except for the first year. The progression rate for grade $j$ in year $t$ equals

$$
R_{j, t}=G_{j, t} / G_{j-1, t-1}
$$

Step 2. Produce a projected progression rate for each of grades 2 through 12. Projections for each grade's progression rate were then produced for the forecast period using single exponential smoothing. A separate smoothing constant, chosen to minimize the sum of squared forecast errors, was used to calculate the projected progression rate for each grade. Single exponential smoothing produces a single forecast for all years in the forecast period. Therefore, for each grade $j$, the projected progression rate, $\hat{R}_{j}$, is the same for each year in the forecast period.

Step 3. Calculate enrollment projections for each of grades 2 through 12. For the first year in the forecast period, $T$, enrollment projections, $\hat{\mathrm{G}}_{j, \mathrm{t}}$, for grades 2 through 12 , were produced using the projected progression rates and the enrollments of grades 1 through 11 from the last year of actual data, $T-1$. Specifically,

$$
\hat{G}_{j, T}=\hat{R}_{j} \cdot G_{j-1, T-1}
$$

This same procedure was then used to produce the projections for the following year, $T+1$, except that enrollment projections for year $T$ were used rather than actual numbers:

$$
\hat{G}_{j, T+1}=\hat{R}_{j} \cdot \hat{G}_{j, T}
$$

The enrollment projections for grades 2 through 11 for year $T$ were those just produced using the grade progression method. The projection for grade 1 for year $T$ was produced using the enrollment rate method, as outlined in steps 4 and 5 below.

The same procedure was used for the remaining years in the projections period.

Step 4. For the last year of actual data, calculate enrollment rates for prekindergarten, kindergarten, grade 1, elementary special and ungraded, and secondary special and ungraded. The first step in projecting prekindergarten, kindergarten, first-grade, elementary special and ungraded, and secondary special and ungraded enrollments using the enrollment rate method was to calculate enrollment rates for each enrollment category for the last year in the sample period, $T-1$, where:

$$
\begin{aligned}
& R N_{T-1}=N_{T-1} / P_{5, T-1} \\
& R K_{T-1}=K_{T-1} / P_{5, T-1} \\
& R G_{1, T-1}=G_{1, T-1} / P_{6, T-1} \\
& R E_{T-1}=E_{T-1} / \Sigma_{i=5}^{13} P_{i, T-1} \\
& R S_{T-1}=S_{T-1} / \sum_{i=14}^{17} P_{i, T-1}
\end{aligned}
$$

These enrollment rates were then used as the projected enrollment rates for each year in the sample period $(\widehat{R N}, \widehat{R K}, \widehat{R G}, \widehat{R E}$, and $\widehat{R S}$.) 
Step 5. Using the rates for the last year of actual data as the projected enrollment rates, calculate enrollment projections for prekindergarten through grade 1 and the ungraded categories. For each year in the forecast period, the enrollment rates were then multiplied by the appropriate population projections from the U.S. Census Bureau $\left(\hat{P}_{\mathrm{i}, t}\right)$ to calculate enrollment projections for prekindergarten (nursery school) $\left(\hat{N}_{t}\right)$, kindergarten $\left(\hat{K}_{t}\right)$, first grade $\left(\hat{G}_{1, t}\right)$, elementary ungraded $\left(\hat{E}_{t}\right)$, and secondary ungraded $\left(\hat{S}_{t}\right)$

$$
\begin{aligned}
& \hat{N}_{t}=\widehat{R N} \cdot \hat{P}_{5, t} \\
& \hat{K}_{t}=\widehat{R K} \cdot \hat{P}_{5, t} \\
& \hat{G}_{1, t}=\widehat{R G} \cdot \hat{P}_{5, t} \\
& \hat{E}_{t}=\widehat{R E} \cdot\left(\sum_{i=5}^{13} \hat{P}_{i, t}\right) \\
& \hat{S}_{t}=\widehat{R S} \cdot\left(\sum_{i=14}^{17} \hat{P}_{i, t}\right)
\end{aligned}
$$

Step 6. Calculate total elementary and secondary enrollments by summing the projections for each grade and the ungraded categories. To obtain projections of total enrollment, projections of enrollments for the individual grades (prekindergarten through 12), elementary ungraded, and secondary ungraded were summed.

\section{National Elementary and Secondary Enrollment Model}

This model was used to project national total, public, and private school enrollments by grade level and for ungraded elementary and ungraded secondary programs. National enrollment projections for public and private schools were developed separately, then added together to yield total elementary and secondary enrollment projections for the nation. To develop these projections, enrollment data from NCES were used, along with population estimates and projections from the U.S. Census Bureau. Below is information about the specific data used to develop the public school projections and the private school projections, as well as information about the grade progression rates and enrollment rates specific to public schools and private schools.

For details on procedures used to develop the projections, see "Procedures and equations used in all three elementary and secondary enrollment models, "earlier in this section of appendix A.

\section{Data used to develop national elementary and secondary enrollment projections}

Public school enrollment data. Public school enrollment data from the NCES Common Core of Data (CCD) for 1972 to 2008 were used to develop the national public school enrollment projections.

Private school enrollment data. Private school enrollment data from the NCES Private School Universe Survey (PSS) for 1989-90, 1991-92, 1993-94, 1995-96, 1997-98, 1999-2000, 2001-02, 2003-04, 2005-06, 2007-08, and 2009-10 were used to develop the national private school enrollment projections. Since the PSS is collected in the fall of odd numbered years, data for even numbered years without a PSS collection were estimated by interpolating grade-by-grade progression data from PSS.

Population estimates and projections used for public school enrollment projections. Population estimates for 1972 to 2009 and population projections for 2010 to 2020 from the U.S. Census Bureau were also used to develop the public school enrollment projections. The set of population projections used in this year's Projections of Education Statistics are the Census Bureau's 2008 National Population Projections by age and sex (August 2008), adjusted to line up with the most recent historical estimates. This was done through the use of ratio adjustments in which, for each combination of state, age, and sex, the population projections from 2010 to 2020 were multiplied by the ratio of the population estimate for 2009 to the population projection for 2009 .

Population estimates and projections used for private school enrollment projections. Population estimates for 1989 to 2009 and population projections for 2010 to 2020 from the U.S. Census Bureau were used to develop the private school enrollment projections. The population projections were ratio-adjusted to line up with the most recent historical estimates.

\section{Grade progression and enrollment rates for national elementary and secondary enrollment projections}

Public school grade progression and enrollment rates. Table A-5 on page 91 shows the public school grade progression rates for 2008 and projections for 2009 through 2020. Table A-6 on page 91 shows the public school enrollment rates for 2008 and projections for 2009 through 2020. 


\section{Accuracy of national elementary and secondary enrollment projections}

Mean absolute percentage errors (MAPEs) for projections of public school enrollment were calculated using the last 27 editions of Projections of Education Statistics, while MAPEs for projections of private school enrollment were calculated using the last nine editions. Table A, below, shows MAPEs for both public and private school enrollment projections.

Table A. Mean absolute percentage errors (MAPEs) of enrollment projections, by lead time, control of school, and grade in elementary and secondary schools: 2011

\begin{tabular}{|c|c|c|c|c|c|c|c|c|c|c|}
\hline \multirow[b]{2}{*}{ Statistic } & \multicolumn{10}{|c|}{ Lead time (years) } \\
\hline & 1 & 2 & 3 & 4 & 5 & 6 & 7 & 8 & 9 & 10 \\
\hline \multicolumn{11}{|c|}{ Public elementary and secondary schools } \\
\hline Prekindergarten-12 enrollment & 0.3 & 0.6 & 0.8 & 1.1 & 1.3 & 1.5 & 1.7 & 2.0 & 2.2 & 2.4 \\
\hline Prekindergarten-8 enrollment & 0.4 & 0.7 & 1.0 & 1.2 & 1.3 & 1.7 & 2.0 & 2.5 & 2.8 & 3.2 \\
\hline 9-12 enrollment & 0.3 & 0.6 & 0.9 & 1.1 & 1.3 & 1.5 & 1.9 & 2.3 & 2.4 & 2.3 \\
\hline \multicolumn{11}{|c|}{ Private elementary and secondary schools } \\
\hline Prekindergarten-12 enrollment & 3.4 & 4.6 & 3.7 & 7.2 & 7.7 & 10.6 & 9.3 & 9.4 & 8.1 & 6.3 \\
\hline Prekindergarten-8 enrollment & 3.5 & 4.9 & 4.1 & 8.0 & 9.2 & 12.1 & 10.6 & 10.4 & 10.2 & 7.9 \\
\hline 9-12 enrollment & 3.0 & 3.8 & 2.3 & 4.3 & 2.8 & 5.8 & 5.7 & 6.1 & 1.3 & 1.3 \\
\hline
\end{tabular}

NOTE: Mean absolute percentage error is the average value over past projections of the absolute values of errors expressed in percentage terms. MAPEs for public PK-12 enrollments were calculated using the last 27 editions of Projections of Education Statistics. MAPEs for private PK-12 enrollments were calculated from the past nine editions. Calculations were made using unrounded numbers. Some data have been revised from previously published figures.

SOURCE: U.S. Department of Education, National Center for Education Statistics, Projections of Education Statistics, various issues. (This table was prepared March 2011.)

For more information about MAPEs, see Section A.O. Introduction, earlier in appendix A.

\section{State Public Elementary and Secondary Enrollment Model}

This edition of Projections of Education Statistics contains projected trends in public elementary and secondary enrollment by grade level from 2009 to 2020 for each of the 50 states and the District of Columbia, as well as for each region of the country. The state enrollment projections were produced in two stages:

» first, an initial set of projections for each state was produced; and

» second, these initial projections were adjusted to sum to the national public enrollment totals produced by the National Elementary and Secondary Enrollment Model.

For each region, the enrollment projections equaled the sum of enrollment projections for the states within that region.

\section{Initial set of state projections}

The same methods used to produce the national enrollment projections—-namely, the grade progression rate method and the enrollment rate method-were used to produce the initial sets of public school enrollment projections for each state and the District of Columbia. A separate smoothing constant, chosen to minimize the sum of squared forecast errors, was used to calculate the projected progression rate for each combination of jurisdiction and grade.

For details on the procedures used to develop the initial sets of projections, see "Procedures and equations used in all three elementary and secondary enrollment models, "earlier in this section of appendix A.

\section{Limitations of the grade progression method for state projections}

The grade progression rate method assumes that past trends in factors affecting public school enrollments will continue over the forecast period. This assumption implies that all factors influencing enrollments will display future patterns consistent with past patterns. Therefore, this method has limitations when applied to states with unanticipated changes in migration rates. This method implicitly includes the net effect of such factors as migration, dropouts, deaths, nonpromotion, and transfers to and from private schools. 


\section{Adjustments to the state projections}

The initial projections of state public school enrollments were adjusted to sum to the national projections of public school prekindergarten $(\mathrm{PK})-12, \mathrm{PK}-8$, and 9-12 enrollments shown in table 1 on page 31 . This was done through the use of ratio adjustments in which all the states' initial enrollment projections for each grade level were multiplied by the ratio of the national enrollment projection for that grade level to the sum of the state enrollment projections for that grade level.

\section{Data used to develop state elementary and secondary enrollment projections}

Public school enrollment data. Public school enrollment data from the NCES Common Core of Data (CCD) for 1980 to 2008 were used to develop these projections.

Population estimates and projections. Population estimates for 1980 to 2009 and population projections for 2010 to 2020 from the U.S. Census Bureau were used to develop the state-level enrollment projections. The set of population projections used in this year's Projections of Education Statistics are the Census Bureau's set of interim state-level population projections by age and sex (April 2005). In order for the state-level population projections to be consistent with the most recent historical estimates released by the Census Bureau, these projections were adjusted to line up with the most recent historical estimate for each state. This was done through the use of ratio adjustments in which, for each combination of state, age, and sex, the population projections from 2010 to 2020 were multiplied by the ratio of the population estimate for 2009 to the population projection for 2009.

\section{Accuracy of state elementary and secondary enrollment projections}

Mean absolute percentage errors (MAPEs) for projections of public school enrollment by state were calculated using the last 15 editions of Projections of Education Statistics. Tables A-7 through A-9 on pages 92-97 show MAPEs for PK-12, PK-8, and 9-12 enrollment in public elementary and secondary schools by state.

\section{National Public Elementary and Secondary Enrollment by Race/Ethnicity Model}

This edition of Projections of Education Statistics contains projected trends in national public elementary and secondary enrollment by race/ethnicity from 2009 to 2020 . The enrollment projections by race/ethnicity were produced in two stages:

» first, an initial set of projections by race/ethnicity was produced; and

» second, these initial projections were adjusted to sum to the national totals.

\section{Initial set of projections by race/ethnicity}

The same methods used to produce the national enrollment projections-namely, the grade progression rate method and the enrollment rate method-were used to produce initial sets of projections for each of the following five racial/ethnic groups: White, Black, Hispanic, Asian/Pacific Islander, and American Indian/Alaska Native. A separate smoothing constant, chosen to minimize the sum of squared forecast errors, was used to calculate the projected progression rate for each combination of race/ethnicity and grade.

For details on the procedures used to develop the initial sets of projections, see "Procedures and equations used in all three elementary and secondary enrollment models, "earlier in this section of appendix $A$.

\section{Adjustments to the projections by race/ethnicity}

The initial projections of enrollments by race/ethnicity were adjusted to sum to the national projections of public school PK-12, PK-8, and 9-12 enrollments shown in table 1 on page 31. This was done through the use of ratio adjustments in which all the initial enrollment projections by race/ethnicity for each grade level were multiplied by the ratio of the national enrollment projection for that grade level to the sum of the initial enrollment projections by race/ethnicity for that grade level. 


\section{Data and imputations used to develop enrollment projections by race/ethnicity}

Public school enrollment data. Public school enrollment data by grade level and race/ethnicity from the NCES Common Core of Data (CCD) for 1994 to 2008 were used to develop these projections. While projections by race/ethnicity were produced at the national level only, the national data used to develop these projections were constructed from state-level data on enrollment by grade level and race/ethnicity. In those instances where states did not report their enrollment data by grade level and race/ethnicity, the state-level data had to be examined and some imputations made in order to produce the national public school enrollment by grade level and race/ethnicity data. For example, in 1994, North Dakota did not report gradelevel enrollment data by race/ethnicity. It did, however, report these numbers for 1995 . So, to impute these numbers for 1994, North Dakota's 1994 grade-level enrollment data were multiplied by the state's $1995 \mathrm{racial} /$ ethnic breakdowns at each grade level. In 2008, jurisdictions could classify students by an additional racial/ethnic group - those of two or more races. As only five states used this reporting category, those numbers were proportioned among the other five racial/ethnic groups by each of the five ethnic groups' shares of total enrollment, excluding enrollment of those of two or more races. When a sufficient number of states use this racial/ethnic group, projections will be developed for this group.

Population estimates and projections. Population estimates for 2000 to 2009 and population projections for 2010 to 2020 from the U.S. Census Bureau were used to develop the enrollment projections by race/ethnicity. The set of population projections used in this year's Projections of Education Statistics are the Census Bureau's 2008 National Population Projections by age, sex, and race/ethnicity (August 2008), ratio-adjusted to line up with the most recent historical estimates.

\section{Accuracy of enrollment projections by race/ethnicity}

Because this is the second edition of Projections of Education Statistics to include projections of elementary and secondary public school enrollments by race/ethnicity, the difference between the projections and actual data for the same years cannot yet be determined. 
Table A-5. Actual and projected national public school grade progression rates: Fall 2008, and fall 2009 through fall 2020

\begin{tabular}{lrr}
\hline Grade & Actual 2008 & Projected 2009 through 2020 \\
\hline 1 to 2 & 98.6 & 98.6 \\
2 to 3 & 100.1 & 100.3 \\
3 to 4 & 99.7 & 100.1 \\
4 to 5 & 100.1 & 100.4 \\
5 to 6 & 100.4 & 100.8 \\
6 to 7 & 100.7 & 100.8 \\
7 to 8 & 99.8 & 99.8 \\
8 to 9 & 111.1 & 111.1 \\
9 to 10 & 91.0 & 91.0 \\
10 to 11 & 91.8 & 91.8 \\
11 to 12 & 95.6 & 95.6
\end{tabular}

NOTE: The progression rate for a particular grade in a year equals the enrollment in the grade for that year divided by the enrollment in the previous grade in the previous year, all multiplied by 100. For example, the progression rate for third-graders in 2008 equals the enrollment of third-graders in 2008 divided by the enrollment of second-graders in 2007, all multiplied by 100.

SOURCE: U.S. Department of Education, National Center for Education Statistics, Common Core of Data (CCD), "State Nonfiscal Survey of Public Elementary/Secondary Education," 2008-09; and National Elementary and Secondary Enrollment Model, 1972-2008. (This table was prepared February 2011.)

Table A-6. Actual and projected national enrollment rates in public schools, by grade level: Fall 2008, and fall 2009 through fall 2020

\begin{tabular}{lrr}
\hline Grade level & Actual 2008 & Projected 2009 through 2020 \\
\hline Prekindergarten & 28.6 & 28.6 \\
Kindergarten & 88.2 & 88.2 \\
Grade 1 & 90.5 & 90.5 \\
Elementary ungraded & 0.3 & 0.3 \\
Secondary ungraded & 0.4 & 0.4
\end{tabular}

NOTE: The enrollment rate for each grade level equals the enrollment at that grade level divided by the population of that grade's base age, all multiplied by 100. The base age for each grade level is as follows: kindergarten, 5 years old; grade 1, 6 years old; elementary ungraded, 5 to 13 years old; and secondary ungraded, 14 to 17 years old. Projected values for 2009 through 2020 were held constant at the actual values for 2008. SOURCE: U.S. Department of Education, National Center for Education Statistics, Common Core of Data (CCD), "State Nonfiscal Survey of Public Elementary/Secondary Education," 2008-09; and National Elementary and Secondary Enrollment Model, 1972-2008. (This table was prepared February 2011.) 
Table A-7. Mean absolute percentage errors (MAPEs) for projected prekindergarten-12 enrollment in public elementary and secondary schools, by lead time, region, and state: 2011

\begin{tabular}{|c|c|c|c|c|c|c|c|c|c|c|}
\hline \multirow[b]{2}{*}{ Region and state } & \multicolumn{10}{|c|}{ Lead time (years) } \\
\hline & 1 & 2 & 3 & 4 & 5 & 6 & 7 & 8 & 9 & 10 \\
\hline United States & 0.3 & 0.6 & 0.8 & 1.1 & 1.3 & 1.5 & 1.7 & 2.0 & 2.2 & 2.4 \\
\hline Northeast & 0.3 & 0.3 & 0.5 & 0.6 & 0.5 & 0.7 & 0.8 & 0.6 & 0.7 & 0.8 \\
\hline Connecticut & 0.6 & 0.8 & 1.1 & 1.5 & 2.2 & 3.1 & 4.1 & 5.5 & 6.3 & 7.2 \\
\hline Maine & 0.8 & 1.4 & 1.5 & 1.5 & 1.9 & 1.9 & 1.7 & 1.6 & 1.9 & 1.9 \\
\hline Massachusetts & 0.4 & 0.3 & 0.4 & 0.3 & 0.5 & 0.8 & 0.9 & 1.1 & 1.4 & 1.9 \\
\hline New Hampshire & 0.6 & 0.7 & 0.9 & 1.3 & 1.4 & 2.0 & 2.5 & 3.0 & 3.2 & 3.3 \\
\hline New Jersey & 0.6 & 1.0 & 1.5 & 2.1 & 2.6 & 3.1 & 3.8 & 4.8 & 5.5 & 6.1 \\
\hline New York & 0.5 & 0.7 & 1.0 & 1.4 & 1.8 & 2.3 & 2.7 & 3.1 & 3.7 & 4.0 \\
\hline Pennsylvania & 0.9 & 1.3 & 1.3 & 1.4 & 1.4 & 1.8 & 1.8 & 1.7 & 1.6 & 2.2 \\
\hline Rhode Island & 1.0 & 1.6 & 2.4 & 2.9 & 3.2 & 3.0 & 2.6 & 1.6 & 1.7 & 2.5 \\
\hline Vermont & 1.0 & 1.5 & 1.9 & 2.3 & 3.0 & 3.6 & 4.5 & 5.6 & 6.6 & 7.4 \\
\hline Midwest & 0.3 & 0.4 & 0.6 & 0.7 & 0.9 & 1.1 & 1.4 & 1.8 & 1.7 & 1.6 \\
\hline Illinois & 0.6 & 0.8 & 1.0 & 1.1 & 1.3 & 1.6 & 2.0 & 2.6 & 3.0 & 3.3 \\
\hline Indiana & 0.3 & 0.7 & 1.0 & 1.5 & 2.0 & 2.5 & 3.0 & 3.0 & 2.6 & 2.4 \\
\hline lowa & 0.6 & 0.7 & 0.9 & 1.0 & 1.4 & 1.5 & 1.0 & 1.3 & 1.7 & 2.2 \\
\hline Kansas & 0.8 & 1.0 & 1.4 & 1.4 & 1.5 & 1.6 & 1.6 & 1.7 & 1.6 & 1.9 \\
\hline Michigan & 0.7 & 1.7 & 2.5 & 3.1 & 3.4 & 3.9 & 3.9 & 3.5 & 4.0 & 4.0 \\
\hline Minnesota & 0.4 & 0.5 & 0.7 & 0.8 & 0.9 & 1.1 & 1.2 & 1.4 & 1.5 & 1.8 \\
\hline Missouri & 0.4 & 0.5 & 0.6 & 0.8 & 1.0 & 1.1 & 1.0 & 1.1 & 0.8 & 1.2 \\
\hline Nebraska & 0.6 & 0.7 & 0.9 & 1.0 & 1.5 & 1.9 & 1.9 & 2.0 & 1.9 & 1.6 \\
\hline North Dakota & 0.7 & 1.2 & 1.6 & 2.2 & 3.0 & 4.2 & 6.0 & 7.7 & 9.5 & 11.1 \\
\hline Ohio & 0.4 & 0.4 & 0.7 & 0.9 & 1.2 & 1.6 & 1.8 & 2.2 & 2.5 & 2.4 \\
\hline South Dakota & 1.2 & 2.2 & 3.4 & 4.5 & 5.7 & 7.0 & 7.8 & 9.0 & 10.4 & 13.2 \\
\hline Wisconsin & 0.7 & 0.9 & 1.2 & 1.4 & 1.3 & 1.4 & 1.7 & 1.9 & 1.7 & 2.0 \\
\hline
\end{tabular}

See notes at end of table. 
Table A-7. Mean absolute percentage errors (MAPEs) for projected prekindergarten-12 enrollment in public elementary and secondary schools, by lead time, region, and state: 2011-Continued

\begin{tabular}{|c|c|c|c|c|c|c|c|c|c|c|}
\hline \multirow[b]{2}{*}{ Region and state } & \multicolumn{10}{|c|}{ Lead time (years) } \\
\hline & 1 & 2 & 3 & 4 & 5 & 6 & 7 & 8 & 9 & 10 \\
\hline United States & 0.3 & 0.6 & 0.8 & 1.1 & 1.3 & 1.5 & 1.7 & 2.0 & 2.2 & 2.4 \\
\hline South & 0.5 & 0.9 & 1.4 & 1.8 & 2.2 & 2.7 & 3.0 & 3.3 & 3.4 & 3.2 \\
\hline Alabama & 0.6 & 0.7 & 1.1 & 1.7 & 2.4 & 3.2 & 3.9 & 5.0 & 6.1 & 7.5 \\
\hline Arkansas & 0.6 & 1.2 & 1.9 & 2.6 & 3.6 & 4.5 & 4.8 & 4.5 & 4.0 & 3.5 \\
\hline Delaware & 0.7 & 1.1 & 1.7 & 2.5 & 3.6 & 4.8 & 5.6 & 6.3 & 6.8 & 7.1 \\
\hline District of Columbia & 5.5 & 4.5 & 6.4 & 7.0 & 6.6 & 7.7 & 7.4 & 5.8 & 7.2 & 4.3 \\
\hline Florida & 1.0 & 1.8 & 2.3 & 2.7 & 3.3 & 4.2 & 5.4 & 7.0 & 8.3 & 9.2 \\
\hline Georgia & 0.8 & 1.3 & 1.8 & 2.3 & 2.8 & 3.6 & 4.2 & 4.6 & 5.0 & 5.6 \\
\hline Kentucky & 1.6 & 1.6 & 2.2 & 2.5 & 2.4 & 3.2 & 3.1 & 2.6 & 3.5 & 3.3 \\
\hline Louisiana & 2.2 & 3.4 & 4.1 & 4.1 & 4.8 & 5.7 & 7.1 & 7.2 & 9.0 & 11.0 \\
\hline Maryland & 0.5 & 0.7 & 1.0 & 1.3 & 1.7 & 1.9 & 2.3 & 2.4 & 2.6 & 3.2 \\
\hline Mississippi & 0.4 & 1.0 & 1.3 & 1.6 & 1.9 & 2.3 & 2.6 & 3.1 & 3.8 & 4.4 \\
\hline North Carolina & 0.9 & 1.3 & 2.0 & 2.9 & 3.8 & 4.6 & 4.9 & 4.9 & 4.9 & 4.7 \\
\hline Oklahoma & 1.0 & 1.4 & 2.1 & 2.8 & 3.6 & 4.3 & 4.7 & 5.2 & 5.3 & 5.1 \\
\hline South Carolina & 0.8 & 1.3 & 1.7 & 2.3 & 3.0 & 3.8 & 4.5 & 5.3 & 5.4 & 4.6 \\
\hline Tennessee & 1.1 & 1.4 & 1.6 & 2.2 & 2.7 & 3.3 & 3.4 & 3.8 & 3.3 & 2.8 \\
\hline Texas & 0.9 & 1.6 & 2.2 & 2.7 & 3.4 & 4.2 & 5.1 & 5.8 & 6.4 & 6.6 \\
\hline Virginia & 0.5 & 0.6 & 0.9 & 1.3 & 1.9 & 2.5 & 2.8 & 3.2 & 3.6 & 3.6 \\
\hline West Virginia & 0.6 & 0.7 & 1.1 & 1.5 & 2.3 & 3.0 & 3.5 & 4.3 & 4.7 & 4.8 \\
\hline West & 0.5 & 1.0 & 1.3 & 1.4 & 1.6 & 1.9 & 2.0 & 1.8 & 1.7 & 1.5 \\
\hline Alaska & 1.1 & 1.8 & 2.4 & 2.8 & 3.5 & 4.9 & 6.5 & 7.6 & 8.7 & 9.7 \\
\hline Arizona & 2.6 & 3.6 & 4.8 & 5.0 & 6.2 & 6.9 & 7.3 & 8.7 & 10.7 & 11.9 \\
\hline California & 0.6 & 1.1 & 1.6 & 2.0 & 2.4 & 2.9 & 2.8 & 2.1 & 1.9 & 2.4 \\
\hline Colorado & 0.6 & 0.9 & 1.3 & 1.8 & 2.4 & 3.1 & 4.0 & 4.7 & 5.3 & 5.9 \\
\hline Hawaii & 1.8 & 3.0 & 4.2 & 5.8 & 7.5 & 9.8 & 12.5 & 14.6 & 16.5 & 19.2 \\
\hline Idaho & 0.7 & 1.4 & 2.0 & 2.8 & 3.7 & 4.3 & 4.7 & 5.0 & 5.3 & 5.6 \\
\hline Montana & 0.9 & 1.6 & 2.5 & 3.7 & 5.0 & 6.9 & 8.9 & 10.9 & 12.4 & 13.6 \\
\hline Nevada & 1.0 & 1.7 & 2.5 & 3.6 & 5.2 & 7.1 & 9.6 & 12.3 & 14.7 & 17.7 \\
\hline New Mexico & 1.5 & 2.5 & 3.6 & 5.0 & 6.6 & 8.1 & 10.0 & 12.0 & 14.0 & 15.9 \\
\hline Oregon & 0.6 & 1.1 & 1.5 & 1.8 & 2.0 & 2.2 & 2.9 & 3.6 & 3.8 & 4.0 \\
\hline Utah & 1.6 & 1.4 & 1.5 & 2.7 & 3.7 & 4.6 & 5.2 & 5.8 & 6.1 & 5.1 \\
\hline Washington & 0.5 & 0.8 & 1.1 & 1.5 & 1.7 & 1.9 & 2.2 & 2.9 & 3.2 & 4.0 \\
\hline Wyoming & 0.9 & 1.5 & 2.7 & 4.0 & 5.7 & 7.3 & 9.1 & 11.5 & 14.3 & 17.5 \\
\hline
\end{tabular}

NOTE: Mean absolute percentage error is the average value over past projections of the absolute values of errors expressed in percentage terms. National MAPEs for public prekindergarten-12 enrollments were calculated using the last 27 editions of Projections of Education Statistics and state MAPEs were calculated using the last 15 editions of Projections of Education Statistics. Calculations were made using unrounded numbers. Some data have been revised from previously published figures.

SOURCE: U.S. Department of Education, National Center for Education Statistics, Projections of Education Statistics, various issues. (This table was prepared February 2011.) 
Table A-8. Mean absolute percentage errors (MAPEs) for projected prekindergarten-8 enrollment in public elementary and secondary schools, by lead time, region, and state: 2011

\begin{tabular}{|c|c|c|c|c|c|c|c|c|c|c|}
\hline \multirow[b]{2}{*}{ Region and state } & \multicolumn{10}{|c|}{ Lead time (years) } \\
\hline & 1 & 2 & 3 & 4 & 5 & 6 & 7 & 8 & 9 & 10 \\
\hline United States & 0.4 & 0.7 & 1.0 & 1.2 & 1.3 & 1.7 & 2.0 & 2.5 & 2.8 & 3.2 \\
\hline Northeast & 0.3 & 0.4 & 0.6 & 0.6 & 0.4 & 0.6 & 0.7 & 0.5 & 0.6 & 0.7 \\
\hline Connecticut & 0.7 & 1.0 & 1.4 & 1.9 & 2.6 & 3.4 & 4.5 & 5.5 & 5.9 & 6.2 \\
\hline Maine & 0.6 & 1.0 & 1.2 & 1.7 & 2.1 & 2.6 & 3.6 & 4.1 & 4.6 & 4.8 \\
\hline Massachusetts & 0.3 & 0.5 & 0.7 & 0.9 & 0.9 & 1.4 & 1.5 & 1.7 & 1.8 & 2.1 \\
\hline New Hampshire & 0.7 & 0.9 & 1.3 & 2.0 & 2.4 & 3.2 & 4.1 & 4.1 & 3.8 & 3.6 \\
\hline New Jersey & 0.7 & 1.1 & 1.5 & 2.0 & 2.3 & 2.7 & 3.2 & 3.7 & 4.1 & 4.4 \\
\hline New York & 0.5 & 0.7 & 0.9 & 1.4 & 1.6 & 2.1 & 2.5 & 2.9 & 3.4 & 3.8 \\
\hline Pennsylvania & 0.5 & 0.9 & 1.0 & 1.1 & 1.2 & 1.4 & 1.2 & 1.1 & 1.3 & 1.6 \\
\hline Rhode Island & 1.3 & 1.8 & 2.5 & 3.1 & 3.4 & 3.5 & 3.6 & 2.6 & 2.8 & 3.5 \\
\hline Vermont & 1.3 & 1.7 & 2.1 & 2.6 & 3.9 & 5.2 & 7.1 & 8.7 & 9.9 & 10.6 \\
\hline Midwest & 0.3 & 0.3 & 0.6 & 0.7 & 0.7 & 0.8 & 1.0 & 1.3 & 1.3 & 1.3 \\
\hline Illinois & 0.7 & 0.9 & 1.0 & 1.2 & 1.5 & 1.9 & 2.3 & 2.8 & 2.9 & 2.9 \\
\hline Indiana & 0.4 & 0.7 & 1.1 & 1.4 & 1.8 & 2.3 & 2.6 & 2.5 & 2.3 & 2.3 \\
\hline lowa & 0.8 & 0.9 & 1.1 & 1.4 & 1.9 & 2.1 & 1.6 & 1.8 & 2.1 & 2.4 \\
\hline Kansas & 0.9 & 1.1 & 1.3 & 1.3 & 1.6 & 1.8 & 1.9 & 2.2 & 2.4 & 2.7 \\
\hline Michigan & 0.7 & 1.4 & 2.2 & 2.8 & 2.8 & 3.0 & 3.1 & 3.2 & 3.2 & 2.5 \\
\hline Minnesota & 0.4 & 0.5 & 0.7 & 0.9 & 0.9 & 1.1 & 1.0 & 1.0 & 1.0 & 1.3 \\
\hline Missouri & 0.6 & 0.8 & 1.1 & 1.4 & 1.6 & 1.6 & 1.5 & 1.3 & 0.9 & 1.1 \\
\hline Nebraska & 0.7 & 0.9 & 1.0 & 1.1 & 1.6 & 2.0 & 2.0 & 2.2 & 2.2 & 2.1 \\
\hline North Dakota & 1.1 & 1.9 & 2.4 & 3.2 & 4.3 & 5.8 & 8.2 & 10.1 & 11.5 & 12.4 \\
\hline Ohio & 0.4 & 0.5 & 0.6 & 0.7 & 0.7 & 0.9 & 1.0 & 1.2 & 1.4 & 1.6 \\
\hline South Dakota & 1.2 & 2.2 & 3.4 & 4.9 & 6.7 & 8.9 & 10.2 & 12.1 & 13.5 & 14.9 \\
\hline Wisconsin & 0.7 & 0.8 & 1.0 & 1.2 & 1.3 & 1.5 & 1.4 & 1.6 & 1.3 & 1.6 \\
\hline
\end{tabular}

See notes at end of table. 
Table A-8. Mean absolute percentage errors (MAPEs) for projected prekindergarten-8 enrollment in public elementary and secondary schools, by lead time, region, and state: $2011-$ Continued

\begin{tabular}{|c|c|c|c|c|c|c|c|c|c|c|}
\hline \multirow[b]{2}{*}{ Region and state } & \multicolumn{10}{|c|}{ Lead time (years) } \\
\hline & 1 & 2 & 3 & 4 & 5 & 6 & 7 & 8 & 9 & 10 \\
\hline United States & 0.4 & 0.7 & 1.0 & 1.2 & 1.3 & 1.7 & 2.0 & 2.5 & 2.8 & 3.2 \\
\hline South & 0.6 & 1.2 & 1.7 & 2.1 & 2.4 & 3.0 & 3.3 & 3.5 & 3.6 & 3.6 \\
\hline Alabama & 0.7 & 1.1 & 1.7 & 2.3 & 3.1 & 4.0 & 4.8 & 6.3 & 7.9 & 9.4 \\
\hline Arkansas & 0.9 & 1.5 & 2.4 & 3.1 & 4.3 & 5.4 & 5.8 & 5.2 & 4.8 & 4.1 \\
\hline Delaware & 0.9 & 1.5 & 2.2 & 3.2 & 4.3 & 5.6 & 6.2 & 7.0 & 7.6 & 8.4 \\
\hline District of Columbia & 4.7 & 4.7 & 6.1 & 6.5 & 6.2 & 6.8 & 7.2 & 4.6 & 8.2 & 5.9 \\
\hline Florida & 1.2 & 2.2 & 2.9 & 3.3 & 3.9 & 4.8 & 6.4 & 7.8 & 8.9 & 9.6 \\
\hline Georgia & 1.0 & 1.7 & 2.3 & 2.7 & 3.2 & 4.0 & 4.6 & 4.9 & 5.3 & 6.0 \\
\hline Kentucky & 1.7 & 2.1 & 3.0 & 3.2 & 3.2 & 3.3 & 3.1 & 3.1 & 3.5 & 3.6 \\
\hline Louisiana & 2.0 & 3.2 & 3.6 & 3.6 & 4.0 & 4.7 & 6.0 & 5.9 & 7.5 & 9.2 \\
\hline Maryland & 0.5 & 0.7 & 1.1 & 1.6 & 2.2 & 2.6 & 3.3 & 3.9 & 4.6 & 5.5 \\
\hline Mississippi & 0.6 & 1.3 & 1.8 & 2.1 & 2.4 & 2.7 & 2.9 & 3.8 & 4.4 & 4.7 \\
\hline North Carolina & 1.1 & 1.7 & 2.5 & 3.4 & 4.5 & 5.6 & 6.0 & 6.4 & 6.6 & 6.5 \\
\hline Oklahoma & 1.4 & 2.0 & 2.9 & 3.8 & 4.8 & 5.8 & 6.5 & 6.9 & 7.1 & 7.3 \\
\hline South Carolina & 1.1 & 1.6 & 2.1 & 3.1 & 3.7 & 4.6 & 4.9 & 5.7 & 5.9 & 5.3 \\
\hline Tennessee & 0.9 & 1.4 & 1.8 & 2.1 & 2.4 & 2.8 & 2.7 & 2.9 & 2.6 & 2.4 \\
\hline Texas & 1.1 & 2.0 & 2.7 & 3.2 & 3.9 & 4.7 & 5.5 & 6.1 & 6.6 & 6.9 \\
\hline Virginia & 0.6 & 0.9 & 1.1 & 1.4 & 1.8 & 2.6 & 2.9 & 3.2 & 3.3 & 3.3 \\
\hline West Virginia & 0.6 & 0.7 & 1.1 & 1.4 & 2.3 & 3.0 & 3.4 & 4.2 & 4.7 & 4.7 \\
\hline West & 0.6 & 1.1 & 1.5 & 1.5 & 1.6 & 2.0 & 2.1 & 1.9 & 2.0 & 1.9 \\
\hline Alaska & 1.3 & 2.0 & 3.0 & 3.6 & 4.9 & 7.0 & 9.4 & 11.0 & 12.2 & 13.5 \\
\hline Arizona & 2.3 & 2.9 & 3.9 & 4.2 & 5.4 & 7.2 & 7.5 & 9.0 & 10.3 & 11.3 \\
\hline California & 0.9 & 1.5 & 2.0 & 2.4 & 2.9 & 3.5 & 3.3 & 2.5 & 2.3 & 2.7 \\
\hline Colorado & 0.7 & 1.2 & 1.7 & 2.3 & 3.1 & 4.0 & 5.0 & 5.9 & 6.5 & 7.1 \\
\hline Hawaii & 2.0 & 3.4 & 4.7 & 6.6 & 8.7 & 11.7 & 15.3 & 18.0 & 20.0 & 22.7 \\
\hline Idaho & 0.9 & 1.9 & 2.8 & 3.6 & 4.6 & 5.3 & 5.7 & 6.1 & 6.7 & 6.6 \\
\hline Montana & 1.2 & 2.0 & 3.4 & 5.0 & 6.9 & 9.7 & 12.9 & 15.6 & 17.5 & 18.5 \\
\hline Nevada & 1.2 & 2.6 & 4.1 & 5.7 & 7.8 & 10.6 & 14.2 & 17.1 & 19.3 & 21.7 \\
\hline New Mexico & 1.3 & 2.3 & 3.1 & 4.2 & 5.7 & 7.3 & 9.7 & 12.0 & 13.5 & 14.4 \\
\hline Oregon & 0.6 & 1.0 & 1.1 & 1.5 & 1.9 & 2.2 & 2.8 & 3.5 & 3.9 & 4.4 \\
\hline Utah & 1.6 & 1.6 & 2.0 & 2.9 & 4.3 & 5.2 & 5.8 & 6.1 & 6.1 & 4.5 \\
\hline Washington & 0.5 & 0.8 & 1.1 & 1.6 & 2.0 & 2.3 & 2.5 & 3.2 & 3.6 & 4.2 \\
\hline Wyoming & 1.1 & 1.7 & 3.4 & 5.1 & 7.7 & 10.1 & 12.8 & 16.0 & 18.7 & 21.3 \\
\hline
\end{tabular}

NOTE: Mean absolute percentage error is the average value over past projections of the absolute values of errors expressed in percentage terms. National MAPEs for public prekindergarten-8 enrollment were calculated using the last 27 editions of Projections of Education Statistics and state MAPEs were calculated using the last 15 editions of Projections of Education Statistics. Calculations were made using unrounded numbers. Some data have been revised from previously published figures.

SOURCE: U.S. Department of Education, National Center for Education Statistics, Projections of Education Statistics, various issues. (This table was prepared February 2011.) 
Table A-9. Mean absolute percentage errors (MAPEs) for projected grades 9-12 enrollment in public schools, by lead time, region, and state: 2011

\begin{tabular}{|c|c|c|c|c|c|c|c|c|c|c|}
\hline \multirow[b]{2}{*}{ Region and state } & \multicolumn{10}{|c|}{ Lead time (years) } \\
\hline & 1 & 2 & 3 & 4 & 5 & 6 & 7 & 8 & 9 & 10 \\
\hline United States & 0.3 & 0.6 & 0.9 & 1.1 & 1.3 & 1.5 & 1.9 & 2.3 & 2.4 & 2.3 \\
\hline Northeast & 0.6 & 0.8 & 0.9 & 1.0 & 1.2 & 1.2 & 1.2 & 1.3 & 1.3 & 1.5 \\
\hline Connecticut & 0.6 & 0.9 & 0.8 & 1.1 & 1.7 & 2.7 & 3.7 & 5.4 & 7.4 & 9.5 \\
\hline Maine & 1.7 & 3.3 & 4.0 & 4.6 & 4.9 & 6.1 & 7.1 & 8.2 & 8.7 & 7.8 \\
\hline Massachusetts & 0.6 & 0.9 & 1.3 & 1.5 & 1.8 & 2.2 & 2.5 & 2.1 & 2.3 & 2.5 \\
\hline New Hampshire & 0.6 & 1.0 & 1.4 & 1.8 & 2.0 & 2.3 & 2.6 & 3.2 & 4.3 & 5.3 \\
\hline New Jersey & 0.6 & 1.4 & 2.1 & 2.6 & 3.4 & 4.7 & 5.8 & 7.3 & 9.3 & 10.4 \\
\hline New York & 0.8 & 1.3 & 1.5 & 1.7 & 2.2 & 2.9 & 3.2 & 3.5 & 4.2 & 4.5 \\
\hline Pennsylvania & 1.8 & 2.2 & 1.9 & 2.2 & 2.4 & 2.9 & 3.1 & 3.1 & 2.4 & 3.7 \\
\hline Rhode Island & 0.8 & 1.5 & 2.4 & 3.5 & 4.5 & 5.0 & 4.6 & 3.5 & 3.1 & 3.6 \\
\hline Vermont & 0.9 & 2.4 & 3.0 & 3.6 & 3.7 & 4.0 & 4.0 & 4.0 & 3.6 & 4.1 \\
\hline Midwest & 0.5 & 0.9 & 1.2 & 1.4 & 1.6 & 1.8 & 2.2 & 2.7 & 2.6 & 2.4 \\
\hline Illinois & 0.9 & 1.2 & 1.6 & 1.8 & 2.2 & 3.0 & 3.6 & 4.4 & 4.9 & 5.5 \\
\hline Indiana & 0.6 & 0.9 & 1.5 & 2.0 & 2.6 & 3.3 & 4.0 & 4.4 & 4.4 & 4.1 \\
\hline lowa & 0.9 & 1.0 & 1.5 & 1.3 & 1.5 & 1.4 & 1.6 & 1.5 & 1.8 & 2.0 \\
\hline Kansas & 1.2 & 1.8 & 2.5 & 2.8 & 2.8 & 2.5 & 2.2 & 2.0 & 1.6 & 0.8 \\
\hline Michigan & 1.6 & 2.9 & 3.5 & 4.1 & 5.1 & 6.4 & 7.3 & 8.1 & 9.3 & 10.0 \\
\hline Minnesota & 0.6 & 1.0 & 1.3 & 1.5 & 1.8 & 2.1 & 2.3 & 2.5 & 2.9 & 3.4 \\
\hline Missouri & 0.4 & 0.7 & 0.8 & 1.3 & 1.6 & 1.6 & 1.8 & 1.7 & 1.4 & 1.4 \\
\hline Nebraska & 0.4 & 0.8 & 1.1 & 1.5 & 1.9 & 2.5 & 3.0 & 3.2 & 3.0 & 2.6 \\
\hline North Dakota & 0.6 & 1.1 & 1.3 & 1.8 & 2.4 & 3.3 & 4.6 & 6.0 & 7.2 & 9.5 \\
\hline Ohio & 0.8 & 1.3 & 1.7 & 2.0 & 2.4 & 3.1 & 3.8 & 4.5 & 4.8 & 4.3 \\
\hline South Dakota & 1.5 & 3.2 & 4.7 & 6.1 & 7.3 & 8.3 & 9.9 & 11.1 & 11.6 & 12.2 \\
\hline Wisconsin & 0.9 & 1.4 & 1.7 & 2.1 & 2.0 & 2.1 & 2.4 & 2.7 & 2.6 & 2.8 \\
\hline
\end{tabular}

See notes at end of table. 
Table A-9. Mean absolute percentage errors (MAPEs) for projected grades 9-12 enrollment in public schools, by lead time, region, and state: 2011-Continued

\begin{tabular}{|c|c|c|c|c|c|c|c|c|c|c|}
\hline \multirow[b]{2}{*}{ Region and state } & \multicolumn{10}{|c|}{ Lead time (years) } \\
\hline & 1 & 2 & 3 & 4 & 5 & 6 & 7 & 8 & 9 & 10 \\
\hline United States & 0.3 & 0.6 & 0.9 & 1.1 & 1.3 & 1.5 & 1.9 & 2.3 & 2.4 & 2.3 \\
\hline South & 0.4 & 0.8 & 1.2 & 1.4 & 1.7 & 2.1 & 2.4 & 2.7 & 2.8 & 2.5 \\
\hline Alabama & 0.9 & 1.1 & 1.6 & 2.0 & 2.2 & 3.2 & 3.7 & 4.0 & 4.4 & 4.5 \\
\hline Arkansas & 0.5 & 0.9 & 1.4 & 1.6 & 1.9 & 2.2 & 2.5 & 2.6 & 2.3 & 2.2 \\
\hline Delaware & 1.3 & 1.2 & 1.5 & 2.0 & 2.8 & 3.7 & 4.4 & 4.6 & 5.5 & 6.3 \\
\hline District of Columbia & 8.1 & 7.4 & 11.1 & 13.9 & 16.5 & 18.8 & 17.5 & 18.5 & 16.9 & 16.0 \\
\hline Florida & 0.7 & 1.1 & 1.4 & 1.7 & 1.9 & 3.0 & 4.2 & 5.3 & 6.9 & 8.3 \\
\hline Georgia & 0.5 & 1.0 & 1.4 & 1.7 & 2.1 & 2.5 & 3.1 & 3.7 & 4.3 & 4.6 \\
\hline Kentucky & 1.7 & 2.2 & 2.4 & 2.5 & 2.1 & 3.7 & 4.1 & 3.6 & 4.0 & 2.9 \\
\hline Louisiana & 3.3 & 4.5 & 5.7 & 5.7 & 7.3 & 8.6 & 10.1 & 10.6 & 13.2 & 16.0 \\
\hline Maryland & 0.6 & 0.7 & 1.0 & 1.3 & 1.3 & 1.6 & 1.8 & 2.1 & 2.4 & 2.3 \\
\hline Mississippi & 0.6 & 1.3 & 1.9 & 2.1 & 2.4 & 2.8 & 3.2 & 3.3 & 3.6 & 4.0 \\
\hline North Carolina & 1.0 & 1.6 & 1.8 & 1.9 & 2.4 & 2.6 & 2.6 & 2.3 & 2.2 & 2.6 \\
\hline Oklahoma & 0.4 & 0.8 & 1.2 & 1.6 & 2.1 & 2.4 & 2.7 & 2.8 & 2.6 & 2.4 \\
\hline South Carolina & 0.8 & 1.3 & 1.6 & 1.6 & 2.0 & 2.6 & 3.4 & 4.4 & 4.7 & 4.6 \\
\hline Tennessee & 2.1 & 2.1 & 3.0 & 4.1 & 4.9 & 5.5 & 5.6 & 6.1 & 4.9 & 4.4 \\
\hline Texas & 0.5 & 1.1 & 1.7 & 2.2 & 2.7 & 3.3 & 4.2 & 5.3 & 5.8 & 5.7 \\
\hline Virginia & 0.5 & 0.9 & 1.3 & 2.1 & 2.9 & 3.5 & 4.0 & 4.4 & 5.0 & 4.6 \\
\hline West Virginia & 0.7 & 1.0 & 1.3 & 1.6 & 2.2 & 3.1 & 3.9 & 4.4 & 4.7 & 5.1 \\
\hline West & 0.6 & 0.8 & 1.3 & 1.6 & 1.9 & 2.0 & 2.3 & 2.6 & 2.5 & 1.4 \\
\hline Alaska & 1.1 & 2.2 & 2.9 & 3.0 & 2.8 & 2.8 & 3.5 & 4.1 & 4.1 & 4.9 \\
\hline Arizona & 4.3 & 6.7 & 9.6 & 8.7 & 8.2 & 6.7 & 6.7 & 7.8 & 11.5 & 14.1 \\
\hline California & 0.5 & 1.1 & 1.6 & 2.1 & 2.5 & 2.6 & 2.6 & 2.6 & 2.4 & 2.3 \\
\hline Colorado & 0.7 & 1.2 & 1.7 & 1.9 & 2.0 & 2.4 & 2.6 & 2.8 & 3.4 & 3.5 \\
\hline Hawaii & 1.9 & 2.8 & 3.8 & 4.6 & 5.4 & 6.5 & 7.5 & 8.6 & 8.7 & 11.0 \\
\hline Idaho & 0.7 & 1.1 & 1.7 & 2.3 & 3.0 & 3.6 & 4.3 & 4.6 & 4.3 & 4.6 \\
\hline Montana & 0.6 & 1.1 & 1.7 & 2.3 & 3.1 & 3.8 & 4.3 & 4.2 & 3.7 & 4.0 \\
\hline Nevada & 1.1 & 2.3 & 2.8 & 2.8 & 3.4 & 3.6 & 4.2 & 5.7 & 8.2 & 11.3 \\
\hline New Mexico & 2.9 & 4.9 & 6.4 & 7.8 & 9.9 & 11.3 & 12.9 & 14.3 & 15.3 & 19.4 \\
\hline Oregon & 1.2 & 1.9 & 2.8 & 3.1 & 3.0 & 3.3 & 4.0 & 5.1 & 5.1 & 4.6 \\
\hline Utah & 1.8 & 1.2 & 0.8 & 2.4 & 2.4 & 3.1 & 3.9 & 4.9 & 6.0 & 6.7 \\
\hline Washington & 0.7 & 0.9 & 1.2 & 1.7 & 1.9 & 2.4 & 3.1 & 3.6 & 4.1 & 4.4 \\
\hline Wyoming & 0.8 & 1.3 & 2.2 & 3.2 & 4.1 & 5.1 & 6.3 & 7.5 & 8.5 & 10.3 \\
\hline
\end{tabular}

NOTE: Mean absolute percentage error is the average value over past projections of the absolute values of errors expressed in percentage terms. National MAPEs for public 9-12 enrollments were calculated using the last 27 editions of Projections of Education Statistics and state MAPEs were calculated using the last 15 editions of Projections of Education Statistics. Calculations were made using unrounded numbers. Some data have been revised from previously published figures.

SOURCE: U.S. Department of Education, National Center for Education Statistics, Projections of Education Statistics, various issues. (This table was prepared February 2011.) 


\section{Projections in this edition}

This edition of Projections of Education Statistics presents projected trends in the number of high school graduates from 2008-09 to 2020-21. These projections were made using three models:

» The National High School Graduates Model was used to project the number of public high school graduates, the number of private high school graduates, and the total number of high school graduates for the nation.

» The State Public High School Graduates Model was used to project the number of public high school graduates for individual states and regions.

» The National Public High School Graduates by Race/Ethnicity Model was used to project the number of public high school graduates for the nation by race/ethnicity.

\section{Overview of approach}

All the high school graduates models first calculated the number of high school graduates as a percentage of grade 12 enrollment based on historical data. Single exponential smoothing was used to project this percentage. The projected percentage was then applied to projections of grade 12 enrollment.

\section{Assumptions underlying this approach}

The percentage of 12th-graders who graduate was assumed to remain constant at levels consistent with the most recent rates. This methodology assumes that past trends in factors affecting graduation rates, such as dropouts, migration, and public or private transfers, will continue over the forecast period. (No specific assumptions were made regarding the dropout rate; the effect of the 12th grade dropout proportion is reflected implicitly in the graduate proportion.) In addition to student behaviors, the projected number of graduates could be affected by changes in graduation requirements, but this is not considered in the projections in this report.

For more details on the steps used for projections of high school graduates, see "Procedures used in all three high school graduates models," below.

\section{Procedures used in all three high school graduates models}

The following steps were used to project the numbers of high school graduates:

Step 1. For each year in the historic period, express the number of high school graduates as a percentage of grade 12 enrollment. This value represents the approximate percentage of 12th-graders who graduate. For information about the specific historical data and analysis periods used for the National High School Graduates Model, the State Public High School Graduates Model, and the National Public High School Graduates by Race/Ethnicity Model, see the description of the appropriate model, later in this section of appendix A.

Step 2. Project the percentage of 12th-graders who graduate from step 1 . This percentage was projected using single exponential smoothing with a smoothing constant chosen to minimize the sum of squared forecast errors. Because single exponential smoothing produces a single forecast for all years in the forecast period, the same projected percentage of grade 12 enrollment was used for each year in the forecast period.

Step 3. Calculate projections of the numbers of high school graduates. For each year in the forecast period, the projected percentage from step 2 was applied to projections of grade 12 enrollment to yield projections of high school graduates.

\section{National High School Graduates Model}

This model was used to project the number of public high school graduates, the number of private high school graduates, and the total number of high school graduates for the nation. Public and private high school graduates were projected separately. The public and private projections were then summed to yield projections of the total number of high school graduates for the nation.

For details of the procedures used to develop the projections, see "Procedures used in all three high school graduates models," above. 


\section{Data used in the National High School Graduates Model}

Public school data on graduates and grade 12 enrollment. Data on public school 12th-grade enrollments and high school graduates from the NCES Common Core of Data (CCD) for 1972-73 to 2007-08 were used to develop national projections of public high school graduates.

Private school data on graduates and grade 12 enrollment. Data on private school 12th-grade enrollments for 1989-90 through 2009-10 and high school graduates for 1988-89 through 2008-09 were used to develop national projections of private high school graduates. The data were from the biennial NCES Private School Universe Survey (PSS) from 1989-90 to 2009-10 with data for 12th grade enrollment the same as the year of the survey and the data for high school graduates for the preceding year (i.e. the 2009-10 PSS presents high school graduates for 2008-09). Since the PSS is collected in the fall of odd numbered years, data for missing years were estimated using data from the PSS. For 12th grade enrollment, estimates for missing years were linear interpolations of the prior year's and succeeding year's actual values. For high school graduates, estimates for the missing years were the interpolations of the high school graduates to estimated 12th grade enrollment percentages for the prior and succeeding years multiplied by the estimated enrollments for the current year.

Public and private school enrollment projections for grade 12. Projections of grade 12 enrollment in public schools and in private schools were used to develop projections of public high school graduates and private high school graduates, respectively. The grade 12 enrollment projections were made using the grade progression method. For more information, see Section A.1. Elementary and Secondary Enrollment, earlier in this appendix.

\section{Accuracy of national high school graduates projections}

Mean absolute percentage errors (MAPEs) for projections of graduates from public high schools were calculated using the last 20 editions of Projections of Education Statistics, while MAPEs for projections of graduates from private high schools were calculated using the last nine editions. Table B, below, shows MAPEs for both public and private school graduation projections.

Table B. Mean absolute percentage errors (MAPEs) of projections of high school graduates, by lead time and control of school: 2011

\begin{tabular}{|c|c|c|c|c|c|c|c|c|c|c|}
\hline \multirow[b]{2}{*}{ Statistic } & \multicolumn{10}{|c|}{ Lead time (years) } \\
\hline & 1 & 2 & 3 & 4 & 5 & 6 & 7 & 8 & 9 & 10 \\
\hline Public high school graduates & 1.0 & 1.0 & 1.5 & 1.7 & 1.6 & 2.1 & 2.8 & 3.7 & 4.2 & 4.0 \\
\hline Private high school graduates & 0.9 & 0.9 & 1.6 & 2.8 & 5.0 & 6.2 & 4.9 & 4.8 & 1.6 & 1.6 \\
\hline
\end{tabular}

NOTE: MAPEs for public high school graduates were calculated from the past 20 editions of Projections of Education Statistics. MAPEs for private high school graduates were calculated from the past nine editions. Calculations were made using unrounded numbers. Some data have been revised from previously published figures.

SOURCE: U.S. Department of Education, National Center for Education Statistics, Projections of Education Statistics, various issues. (This table was prepared March 2011.)

For more information about MAPEs, see Section A.O. Introduction, earlier in appendix A.

\section{State Public High School Graduates Model}

This edition of Projections of Education Statistics contains projections of public high school graduates from 2008-09 to 2020-21 for each of the 50 states and the District of Columbia, as well as for each region of the country. The state projections of high school graduates were produced in two stages:

» first, an initial set of projections for each state was produced; and

» second, these initial projections were adjusted to sum to the national public school totals produced by the National High School Graduates Model.

For each region, the high school graduate projections equaled the sum of high school graduate projections for the states within that region. 


\section{Initial set of state projections}

The same steps used to produce the national projections of high school graduates were used to produce an initial set of projections for each state and the District of Columbia. A separate smoothing constant, chosen to minimize the sum of squared forecast errors, was used to calculate the projected percentage of 12th grade enrollment for each jurisdiction.

For details on the steps used to develop the initial sets of projections, see "Procedures used in all three high school graduate models," earlier in this section of appendix $A$.

\section{Adjustments to the state projections}

The initial projections of state public high school graduates were adjusted to sum to the national projections of public high school graduates shown in table 12 on page 47 . This was done through the use of ratio adjustments in which all the states' high school graduate projections were multiplied by the ratio of the national public high school graduate projection to the sum of the state public high school projections.

\section{Data used in the State Public High School Graduates Model}

Public school data on graduates and grade 12 enrollment at the state level. State-level data on public school 12th-grade enrollments and high school graduates from the NCES Common Core of Data (CCD) for 1980-81 to 2007-08 were used to develop these projections.

Public school projections for grade 12 enrollment at the state level. State-level projections of grade 12 enrollment in public schools were used to develop the state-level projections of public high school graduates. The grade 12 enrollment projections were made using the grade progression method. For more information, see Section A.1. Elementary and Secondary Enrollment, earlier in this appendix.

\section{Accuracy of state public high school graduate projections}

Mean absolute percentage errors (MAPEs) for projections of the number of public high school graduates by state were calculated using the last 15 editions of Projections of Education Statistics. Table A-10 on pages 102-103 show MAPEs for the number of high school graduates by state.

\section{National Public High School Graduates by Race/Ethnicity Model}

The projections of public high school graduates by race/ethnicity were produced in two stages:

» first, an initial set of projections for each racial/ethnic group was produced; and

» second, these initial projections were adjusted to sum to the national public school totals produced by the National High School Graduates Model.

\section{Initial set of projections by race/ethnicity}

The same steps used to produce the national projections of high school graduates were used to produce an initial set of projections for each of the following five racial/ethnic groups: White, Black, Hispanic, Asian/Pacific Islander, and American Indian/Alaska Native. For example, the number of White public high school graduates was projected as a percentage of White grade 12 enrollment in public schools. A separate smoothing constant, chosen to minimize the sum of squared forecast errors, was used to calculate the projected percentage of 12th-grade enrollment for each racial/ethnic group.

\section{Adjustments to the projections by race/ethnicity}

The projections of public high school graduates by race/ethnicity were adjusted to sum to the national projections of public high school graduates shown in table 12 on page 47. This was done through the use of ratio adjustments in which all high school graduate projections by race/ethnicity were multiplied by the ratio of the national high school graduate projection to the sum of the high school projections by race/ethnicity. 


\section{Data and imputations used in the Public High School Graduates by Race/Ethnicity Model}

Public school data on graduates and grade 12 enrollment by race/ethnicity. Data on public high school graduates and grade 12 enrollment by race/ethnicity from the NCES Common Core of Data (CCD) for 1994-95 to 2007-08 were used to develop these projections. In those instances where states did not report their high school graduate data by race/ethnicity, the state-level data had to be examined and some imputations made. For example, in 1994, Arizona did not release high school graduate data by race/ethnicity. It did, however, release grade 12 enrollment numbers by race/ethnicity for that year. So, to impute the high school graduate numbers by race/ethnicity for that year, Arizona's total number of high school graduates for 1994 was multiplied by the state's $1994 \mathrm{racial} / \mathrm{ethnic}$ breakdowns for grade 12 enrollment.

Public enrollment projections for grade 12 by race/ethnicity. Projections of grade 12 enrollment in public schools by race/ ethnicity were used to develop the projections of public high school graduates by race/ethnicity. The grade 12 enrollment projections were made using the grade progression method. For more information, see Section A.1. Elementary and Secondary Enrollment, earlier in this appendix.

\section{Accuracy of enrollment projections by race/ethnicity}

Because this is the second edition of Projections of Education Statistics to include projections of public high school graduates by race/ethnicity, the difference between the projections and actual data for the same years cannot yet be determined. 
Table A-10. Mean absolute percentage errors (MAPEs) for the projected number of high school graduates in public schools, by lead time, region, and state: 2011

\begin{tabular}{|c|c|c|c|c|c|c|c|c|c|c|}
\hline \multirow[b]{2}{*}{ Region and state } & \multicolumn{10}{|c|}{ Lead time (years) } \\
\hline & 1 & 2 & 3 & 4 & 5 & 6 & 7 & 8 & 9 & 10 \\
\hline United States & 1.0 & 1.0 & 1.5 & 1.7 & 1.6 & 2.1 & 2.8 & 3.7 & 4.2 & 4.0 \\
\hline Northeast & 1.3 & 1.6 & 1.5 & 1.5 & 1.8 & 2.3 & 2.4 & 2.9 & 3.3 & 3.5 \\
\hline Connecticut & 1.6 & 1.3 & 1.2 & 2.0 & 2.5 & 2.7 & 3.3 & 2.9 & 3.8 & 3.9 \\
\hline Maine & 2.9 & 4.6 & 3.9 & 4.5 & 4.9 & 5.4 & 6.7 & 7.3 & 8.3 & 9.6 \\
\hline Massachusetts & 0.8 & 1.4 & 2.1 & 2.2 & 2.1 & 2.0 & 2.3 & 1.1 & 1.1 & 1.4 \\
\hline New Hampshire & 1.1 & 1.9 & 2.1 & 2.4 & 2.6 & 3.5 & 3.8 & 4.4 & 4.0 & 3.8 \\
\hline New Jersey & 2.5 & 4.4 & 5.5 & 5.1 & 5.2 & 6.4 & 7.2 & 8.9 & 10.8 & 11.8 \\
\hline New York & 2.0 & 3.0 & 2.6 & 2.9 & 3.6 & 4.2 & 4.9 & 6.0 & 5.6 & 6.0 \\
\hline Pennsylvania & 1.7 & 2.2 & 1.4 & 1.7 & 1.7 & 2.2 & 2.4 & 3.1 & 3.5 & 2.9 \\
\hline Rhode Island & 1.4 & 1.1 & 2.0 & 1.9 & 2.4 & 3.2 & 4.7 & 5.4 & 5.2 & 5.1 \\
\hline Vermont & 2.0 & 2.3 & 3.5 & 4.5 & 6.2 & 6.3 & 6.9 & 7.6 & 8.2 & 8.3 \\
\hline Midwest & 1.0 & 0.7 & 1.6 & 1.5 & 2.0 & 2.4 & 2.3 & 2.8 & 2.3 & 1.9 \\
\hline Illinois & 2.7 & 2.2 & 3.3 & 4.2 & 4.4 & 3.8 & 6.2 & 4.6 & 6.6 & 8.1 \\
\hline Indiana & 1.8 & 2.1 & 1.8 & 1.7 & 1.8 & 2.4 & 3.5 & 3.8 & 4.5 & 3.8 \\
\hline lowa & 1.7 & 1.2 & 2.0 & 1.8 & 2.6 & 3.0 & 2.9 & 2.6 & 3.0 & 2.6 \\
\hline Kansas & 1.4 & 1.4 & 2.0 & 1.9 & 2.9 & 3.9 & 4.5 & 4.8 & 5.3 & 4.6 \\
\hline Michigan & 3.5 & 4.5 & 5.4 & 6.6 & 6.7 & 6.9 & 8.5 & 8.6 & 8.1 & 8.5 \\
\hline Minnesota & 2.5 & 1.5 & 1.5 & 1.8 & 2.1 & 2.1 & 2.7 & 3.6 & 4.9 & 5.0 \\
\hline Missouri & 1.0 & 1.5 & 2.4 & 2.9 & 3.0 & 3.9 & 4.5 & 4.7 & 4.5 & 4.4 \\
\hline Nebraska & 1.7 & 2.2 & 2.3 & 1.9 & 2.1 & 2.4 & 2.4 & 2.5 & 2.2 & 1.7 \\
\hline North Dakota & 1.2 & 1.5 & 1.8 & 1.9 & 2.2 & 2.7 & 3.5 & 3.4 & 4.6 & 6.5 \\
\hline Ohio & 1.7 & 1.5 & 2.9 & 3.2 & 3.7 & 3.6 & 3.2 & 3.5 & 3.1 & 4.4 \\
\hline South Dakota & 2.5 & 3.1 & 3.5 & 5.9 & 9.6 & 10.8 & 12.7 & 14.3 & 17.3 & 18.1 \\
\hline Wisconsin & 1.4 & 1.7 & 2.7 & 2.8 & 2.9 & 3.7 & 4.0 & 4.6 & 4.4 & 3.8 \\
\hline
\end{tabular}

See notes at end of table. 
Table A-10. Mean absolute percentage errors (MAPEs) for the projected number of high school graduates in public schools, by lead time, region, and state: 2011-Continued

\begin{tabular}{|c|c|c|c|c|c|c|c|c|c|c|}
\hline \multirow[b]{2}{*}{ Region and state } & \multicolumn{10}{|c|}{ Lead time (years) } \\
\hline & 1 & 2 & 3 & 4 & 5 & 6 & 7 & 8 & 9 & 10 \\
\hline United States & 1.0 & 1.0 & 1.5 & 1.7 & 1.6 & 2.1 & 2.8 & 3.7 & 4.2 & 4.0 \\
\hline South & 1.2 & 1.3 & 2.3 & 2.0 & 2.2 & 3.0 & 3.4 & 4.5 & 4.3 & 5.0 \\
\hline Alabama & 3.7 & 3.0 & 2.0 & 4.1 & 4.1 & 4.9 & 5.5 & 5.1 & 4.6 & 5.1 \\
\hline Arkansas & 1.5 & 1.8 & 2.1 & 2.3 & 2.8 & 2.9 & 2.9 & 3.1 & 1.9 & 2.8 \\
\hline Delaware & 2.4 & 3.0 & 4.0 & 5.1 & 3.5 & 4.2 & 4.2 & 5.2 & 4.4 & 5.1 \\
\hline District of Columbia & 6.5 & 6.9 & 11.5 & 13.7 & 15.1 & 17.5 & 17.0 & 19.1 & 14.0 & 17.3 \\
\hline Florida & 1.9 & 4.1 & 5.8 & 3.1 & 4.0 & 4.7 & 6.1 & 7.9 & 7.6 & 6.7 \\
\hline Georgia & 2.0 & 2.5 & 3.2 & 4.8 & 6.4 & 7.2 & 7.6 & 7.5 & 6.8 & 5.9 \\
\hline Kentucky & 2.3 & 3.8 & 3.8 & 4.5 & 5.0 & 5.6 & 6.3 & 4.8 & 4.2 & 4.6 \\
\hline Louisiana & 1.4 & 2.4 & 4.3 & 5.4 & 5.1 & 3.8 & 3.6 & 4.4 & 5.0 & 6.8 \\
\hline Maryland & 1.5 & 1.1 & 1.9 & 1.4 & 1.4 & 1.8 & 2.6 & 3.2 & 2.8 & 3.8 \\
\hline Mississippi & 1.0 & 1.5 & 2.2 & 2.5 & 3.3 & 4.1 & 4.0 & 4.0 & 4.0 & 3.1 \\
\hline North Carolina & 1.8 & 1.9 & 3.2 & 3.5 & 4.2 & 4.3 & 4.0 & 4.6 & 3.9 & 7.0 \\
\hline Oklahoma & 1.5 & 1.6 & 2.0 & 1.7 & 2.2 & 3.0 & 3.3 & 3.1 & 2.0 & 2.4 \\
\hline South Carolina & 1.6 & 3.2 & 2.4 & 3.5 & 4.6 & 5.7 & 5.9 & 6.4 & 4.6 & 4.6 \\
\hline Tennessee & 5.4 & 6.9 & 8.9 & 11.2 & 13.3 & 14.4 & 13.7 & 12.9 & 9.3 & 7.4 \\
\hline Texas & 2.7 & 3.8 & 5.2 & 6.0 & 6.3 & 7.6 & 9.0 & 11.1 & 12.9 & 13.9 \\
\hline Virginia & 1.6 & 2.2 & 2.9 & 3.6 & 4.3 & 4.6 & 4.5 & 4.2 & 5.3 & 5.4 \\
\hline West Virginia & 0.7 & 1.1 & 1.9 & 2.1 & 2.3 & 3.4 & 3.6 & 4.9 & 5.6 & 5.3 \\
\hline West & 1.9 & 2.1 & 2.7 & 2.8 & 2.7 & 2.4 & 2.3 & 2.4 & 3.8 & 2.6 \\
\hline Alaska & 2.4 & 2.4 & 2.6 & 3.6 & 3.8 & 4.9 & 6.0 & 6.2 & 6.4 & 6.0 \\
\hline Arizona & 9.4 & 9.8 & 11.5 & 12.2 & 11.0 & 11.3 & 13.7 & 10.8 & 8.2 & 10.2 \\
\hline California & 2.5 & 2.4 & 2.9 & 3.6 & 3.8 & 4.0 & 4.4 & 3.9 & 5.8 & 5.2 \\
\hline Colorado & 1.9 & 2.1 & 2.6 & 1.7 & 2.2 & 2.2 & 2.4 & 3.4 & 4.5 & 3.5 \\
\hline Hawaii & 4.0 & 4.5 & 5.4 & 6.7 & 9.9 & 10.8 & 13.7 & 15.4 & 18.4 & 18.6 \\
\hline Idaho & 0.9 & 1.3 & 1.2 & 1.5 & 2.0 & 2.9 & 3.4 & 4.2 & 5.9 & 5.1 \\
\hline Montana & 0.9 & 0.9 & 1.5 & 1.4 & 2.5 & 3.9 & 5.1 & 7.0 & 9.7 & 10.7 \\
\hline Nevada & 4.2 & 6.6 & 10.3 & 11.9 & 11.5 & 11.9 & 10.8 & 11.7 & 9.9 & 13.5 \\
\hline New Mexico & 3.6 & 2.8 & 4.6 & 4.3 & 5.7 & 6.2 & 7.1 & 9.0 & 12.0 & 10.7 \\
\hline Oregon & 2.1 & 2.4 & 3.1 & 4.2 & 5.1 & 5.5 & 5.6 & 6.8 & 6.6 & 5.4 \\
\hline Utah & 5.0 & 5.3 & 5.1 & 5.3 & 4.2 & 4.2 & 4.5 & 4.7 & 4.1 & 2.8 \\
\hline Washington & 2.1 & 2.2 & 3.1 & 2.0 & 2.6 & 3.1 & 3.6 & 3.4 & 4.9 & 4.3 \\
\hline Wyoming & 1.6 & 2.1 & 2.2 & 2.8 & 3.9 & 5.4 & 7.2 & 8.2 & 10.7 & 10.5 \\
\hline
\end{tabular}

NOTE: Mean absolute percentage error is the average value over past projections of the absolute values of errors expressed in percentage terms. National MAPEs for public high school graduates were calculated using the last 20 editions of Projections of Education Statistics and state MAPEs were calculated using the last 15 editions of Projections of Education Statistics. Calculations were made using unrounded numbers. Some data have been revised from previously published figures.

SOURCE: U.S. Department of Education, National Center for Education Statistics, Projections of Education Statistics, various issues. (This table was prepared February 2011.) 


\section{Projections in this edition}

This edition of Projections of Education Statistics presents projected trends in elementary and secondary teachers, pupil/teacher ratios, and new teacher hires from 2009 to 2020. These projections were made using two models:

» The Elementary and Secondary Teacher Model was used to project the number of public school teachers, the number of private school teachers, and the total number of teachers for the nation. It was also used to project pupil/teacher ratios for public schools, private schools, and all elementary and secondary schools.

» The New Teacher Hires Model was used to project the number of new teacher hires in public schools, private schools, and all schools.

\section{Overview of approach}

\section{Approach for numbers of teachers and pupil/teacher ratios}

Public schools. Multiple linear regression was used to produce initial projections of public school pupil/teacher ratios separately for elementary and secondary schools. The initial projections of elementary pupil/teacher ratios and secondary pupil/ teacher ratios were applied to enrollment projections to project the numbers of elementary teachers and secondary teachers, which were summed to get the total number of public school teachers. Final projections of the overall public school pupil/ teacher ratios were produced by dividing total projected public school enrollment by the total projected number of teachers.

\section{Assumptions underlying this method}

This method assumes that past relationships between the public school pupil/teacher ratio (the dependent variable) and the independent variables used in the regression analysis will continue throughout the forecast period. For more information about the independent variables, see "Elementary and Secondary Teacher Model," later in this section of appendix A.

Private schools. Private school pupil/teacher ratios were projected by applying each year's projected annual percentage change in the overall public school pupil/teacher ratio to the previous year's private school pupil/teacher ratio. The projected private school pupil/teacher ratios were then applied to projected enrollments at private schools to produce projected numbers of private school teachers.

\section{Assumptions underlying this method}

This method assumes that the future pattern in the trend of private school pupil/teacher ratios will be the same as that for public school pupil/teacher ratios. The reader is cautioned that a number of factors could alter the assumption of constant ratios over the forecast period.

\section{Approach for new teacher hires}

The following numbers were projected separately for public schools and for private schools:

» The number of teachers needed to replace teachers who leave teaching from one year to the next. This number was estimated based on continuation rates of teachers by their age.

» The number of teachers needed to fill openings due to an increase in the size of the teaching workforce from one year to the next. This number was estimated by subtracting the projected number of teachers in one year from the projected number of teachers in the next year.

These two numbers were summed to yield the total number of "new teacher hires" for each control of school—that is, teachers who will be hired in a given year, but who did not teach in that control the previous year. A teacher who moves from one control to the other control (e.g. from a public to private school or from a private to a public school) is considered a new teacher hire, but a teacher who moves from one school to another school in the same control is not considered a new teacher hire.

\section{Elementary and Secondary Teacher Model}

Projections for public schools were produced first. Projections for private schools were produced based partially on input from the public school projections. Finally, the public and private school projections were combined into total elementary and secondary school projections (not shown in the steps below). 


\section{Steps used to project numbers of teachers and pupil/teacher ratios}

Public school teachers. The following steps were used for the public school projections:

Step 1. Produce projections of pupil/teacher ratios for public elementary schools and public secondary schools separately. Two separate equations were used-one for elementary schools and one for secondary schools. The equations for elementary and secondary schools included an AR(1) term for correcting for autocorrelation and the following independent variables:

»Independent variables for public elementary school pupil/teacher ratios-(1) average teacher wage relative to the overall economy-level wage, and (2) level of education revenue from state sources in constant dollars per public elementary student.

»Independent variables for public secondary school pupil/teacher ratios-(1) level of education revenue from state sources in constant dollars per public secondary student, and (2) the number of students enrolled in public secondary schools relative to the secondary school-age population.

To estimate the models, they were first transformed into nonlinear models and then the coefficients were estimated simultaneously by applying a Marquardt nonlinear least squares algorithm to the transformed equation.

For details on the equations, model statistics, and data used to project public school pupil/teacher ratios, see "Data and equations used for projections of teachers and pupil/teacher ratios, "below.

Step 2. Produce projections of the number of teachers for public elementary schools and public secondary schools separately. The projections of the public elementary pupil/teacher ratio and public secondary pupil/teacher ratio were applied to projections of enrollments in elementary schools and secondary schools, respectively, to produce projections of public elementary teachers and public secondary teachers.

Step 3. Produce projections of the total number of teachers for public elementary and secondary schools combined. The projections of public elementary teachers and public secondary teachers were added together to produce the projections of the total number of public elementary and secondary teachers.

Step 4. Produce projections of the pupil/teacher ratio for public elementary and secondary schools combined. The projections of the total number of public elementary and secondary teachers were divided by projections of total enrollment in public elementary and secondary schools to produce projections of the overall pupil/teacher ratio in public elementary and secondary schools.

Private school teachers. The following steps were used for the private school projections:

Step 1. Produce projections of the private school pupil/teacher ratio. First, the projection of the private school pupil/teacher ratio for 2010 was calculated by multiplying the private school pupil/teacher ratio for 2009 (the last year of actual data) by the percentage change from 2008 to 2010 in the public school pupil/teacher ratio. The same method was used to calculate the projections of the private school pupil/teacher ratio for 2011 through 2020. That is, each year's projected annual percentage change in the public school pupil/teacher ratio was applied to the previous year's private school pupil/teacher ratio.

Step 2. Produce projections of the number of private school teachers. The projected pupil/teacher ratios were applied to projected private school enrollments to produce projections of private school teachers from 2010 through 2020.

For information about the private school teacher and enrollment data used for the private school projections, see "Data and equations used for projections of teachers and pupil/teacher ratios," below.

\section{Data and equations used for projections of teachers and pupil/teacher ratios}

Public school data used in these projections were by organizational level (i.e., school level), not by grade level. Thus, secondary school enrollment is not the same as enrollment in grades 9 through 12 because some jurisdictions count some grade 7 and 8 enrollment as secondary. For example, some jurisdictions may have 6-year high schools with grades 7 through 12.

Data used to estimate the equation for public elementary school pupil/teacher ratios. The following data were used to estimate the equation:

» To compute the historical elementary school pupil/teacher ratios-Data on 1973-74 to 2008-09 enrollments in public elementary schools came from the NCES Common Core of Data (CCD). The proportion of public school teachers who taught in elementary schools was taken from the National Education Association and then applied to the total number of public school teachers from the CCD to produce the number of teachers in elementary schools.

» Data on 1973-74 to 2008-09 education revenue from state sources came from the CCD. 
Estimated equation and model statistics for public elementary school pupil/teacher ratios. For the estimated equation and model statistics, see table A-11 on page 109. In the public elementary pupil/teacher ratio equation, the independent variables affect the dependent variable in the expected way:

»As the average teacher wage relative to the overall economy-level wage increases, the pupil/teacher ratio increases; and

» As the level of education revenue from state sources in constant dollars per public elementary student increases, the pupil/teacher ratio decreases.

Data used to project public elementary school pupil/teacher ratios. The estimated equation was run using projected values for teacher salaries and education revenues from state sources from 2009-10 through 2020-21. For more information, see Section A.0. Introduction, earlier in this appendix and Section A.4 Expenditures for Public Elementary and Secondary Education later in this appendix.

Data used to estimate the equation for public secondary school pupil/teacher ratios. The following data were used to estimate the equation:

» To compute the historical secondary school pupil/teacher ratios-Data on 1973-74 to 2008-09 enrollments in public secondary schools came from the NCES Common Core of Data (CCD). The proportion of public school teachers who taught in secondary schools was taken from the National Education Association and then applied to the total number of public school teachers from the CCD to produce the number of teachers in secondary schools.

» Data on 1973-74 to 2008-09 education revenue from state sources came from the CCD.

» To compute the historical secondary school enrollment rate-Data on the secondary school-age population from 1973-74 to 2008-09 came from the U.S. Census Bureau. Data on enrollments in public secondary schools during the same period came from the CCD, as noted above.

Estimated equation and model statistics for public secondary school pupil/teacher ratios. For the estimated equation and model statistics, see table A-11 on page 109. In the public secondary pupil/teacher ratio equation, the independent variables affect the dependent variable in the expected way:

»As enrollment rates (number of enrolled students relative to the school-age population) increase, the pupil/teacher ratio increases; and

»As the level of education revenue per secondary student increases, the pupil/teacher ratio decreases.

Data used to project public secondary school pupil/teacher ratios. The estimated equation was run using projections for education revenues, public secondary enrollments, and secondary school-age populations from 2009-10 through 2019-20. Secondary enrollment projections were derived from the enrollment projections described in Section A.1. Elementary and Secondary Enrollment. Population projections were from the Census Bureau's 2008 National Population Projections by age and sex (August 2008), ratio-adjusted to line up with the most recent historical estimates.

Private school teacher and enrollment data. Private school data for 1989-90, 1991-92, 1993-94, 1995-96, 1997-98, 1999-2000, 2001-02, 2003-04, 2005-06, 2007-08, and 2009-10 came from the biennial NCES Private School Universe Survey (PSS). Since the PSS is collected in the fall of odd numbered years, data for years without a PSS collection were estimated using data from the PSS.

Private school enrollment projections. Private school enrollments from 2009 to 2020 came from the projections described in Section A.1. Elementary and Secondary Enrollment, earlier in this appendix.

\section{Accuracy of projections of numbers of teachers}

Mean absolute percentage errors (MAPEs) for projections of public school teachers were calculated using the last 20 editions of Projections of Education Statistics. Table C, below, shows MAPEs for projections of the numbers of public school teachers. There was a change in the methodology for projecting private school teachers beginning with Projections of Education Statistics to 2017, and therefore there are too few years of data to present the MAPEs for private school teachers. 
Table C. Mean absolute percentage errors (MAPEs) of projections of number of public elementary and secondary teachers, by lead time: 2011

\begin{tabular}{|c|c|c|c|c|c|c|c|c|c|c|}
\hline \multirow[b]{2}{*}{ Statistic } & \multicolumn{10}{|c|}{ Lead time (years) } \\
\hline & 1 & 2 & 3 & 4 & 5 & 6 & 7 & 8 & 9 & 10 \\
\hline Public elementary and secondary teachers & 1.0 & 1.4 & 1.7 & 2.3 & 2.9 & 3.4 & 4.1 & 4.4 & 5.1 & 5.9 \\
\hline
\end{tabular}

NOTE: MAPEs for teachers were calculated from the past 20 editions of Projections of Education Statistics containing teacher projections.

Calculations were made using unrounded numbers. Some data have been revised from previously published figures.

SOURCE: U.S. Department of Education, National Center for Education Statistics, Projections of Education Statistics, various issues. (This table was prepared March 2011.)

For more information about MAPEs, see Section A.O. Introduction, earlier in this appendix.

\section{New Teacher Hires Model}

The New Teacher Hires Model was estimated separately for public and private school teachers. The model produces projections of the number of teachers who were not teaching in the previous year, but who will be hired in a given year.

\section{About new teacher hires}

A teacher is considered to be a new teacher hire for a control of school (public or private) for a given year if the teacher teaches in that control that year but had not taught in that control in the previous year. Included among new teachers hires are: (1) teachers who are new to the profession; (2) teachers who had taught previously but had not been teaching the previous year; and (3) teachers who had been teaching in one control the previous year but have moved to the other control. Concerning the last category, if a teacher moves from one public school to a different public school, that teacher would not be counted as a new teacher hire for the purposes of this model. On the other hand, if a teacher moves from a public school to a private school, that teacher would be counted as a private school new teacher hire, since the teacher did not teach in a private school in the previous year.

The New Teacher Hires Model measures the demand for teacher hires. Due to difficulties in defining and measuring the pool of potential teachers, no attempt was made to measure the supply of new teacher candidates.

\section{Steps used to project numbers of new teacher hires}

The steps outlined below provide a general summary of how the New Teacher Hires Model was used to produce projections of the need for new teacher hires.

For more information about the New Teacher Hires Model, see Hussar (1999).

First, the whole series of steps outlined below was used to produce projections of public school new teacher hires. Then, the same steps were used to produce projections of private school new hires. Finally, the public and private new teacher hires were combined to produce projections of total new teacher hires.

Step 1. Estimate the age distribution of full-time-equivalent (FTE) teachers in 2007. For this estimate, the age distribution of the headcount of school teachers (including both full-time and part-time teachers) in 2007 was applied to the national number of FTE teachers in the same year.

Step 2. Estimate the number of new FTE teacher hires needed to replace those who left teaching between 2007 and 2008. In this step

»Age-specific continuation rates for 2004 were applied to the FTE count of teachers by age for 2007, resulting in estimates of the number of FTE teachers who remained in teaching in 2008 by individual age.

» The FTE teachers who remained in teaching by individual age were summed across all ages to produce an estimate of the total number of FTE teachers who remained teaching in 2008.

» The total number of remaining FTE teachers in 2008 was subtracted from the total FTE teacher count for 2007 to produce the estimated number of FTE teachers who left teaching.

Step 3. Estimate the number of new FTE teacher hires needed due to the overall increase in the teacher workforce between 2007 and 2008. The total number of FTE teachers in 2007 was subtracted from the total number of FTE teachers in 2008 to determine the overall increase in the teaching workforce between 2007 and 2008.

Step 4. Estimate the total number of new FTE teacher hires needed in 2008. The number of FTE teachers who left teaching from step 2 was added to the estimated net change in the number of FTE teachers from step 3 to estimate the total number of new FTE teacher hires needed in 2008. 
Step 5. Estimate the FTE count of teachers by age for 2008. In this step

» The age distribution for the headcount of newly hired teachers in 2007 was applied to the estimated total number of new FTE teacher hires in 2008, resulting in the estimated number of new FTE teacher hires by age.

» For each individual age, the estimated number of new FTE teacher hires was added to the estimated number of remaining FTE teachers (from step 2, first bullet) to produce the estimated FTE count of teachers by age for 2008.

Step 6. Repeat steps 2 to 5 for each year from 2009 through 2020.

»In step 2

- For public school teachers ages 22 through 66 and private school teachers ages 21 through 65, projections of age-specific continuation rates were used. A separate smoothing constant, chosen to minimize the sum of squared forecast errors, was used to calculate the projected progression rate for each age. (For a general description of the exponential smoothing technique, see Section A.0. Introduction, earlier in this appendix.)

- For all other ages, the age-specific continuation rates for 2008 (the last year of actual data) were used.

»In step 3, projections of the numbers of FTE teachers were used for all years in which there were no actual teacher numbers. The projections of FTE teachers are described under "Elementary and Secondary Teacher Model," earlier in this section of appendix A.

\section{Assumptions underlying this method}

A number of assumptions are made in order to make these projections. They include that (1) the age distribution of FTE teachers in 2007 was similar to that of full-time and part-time teachers in that year (step 1); (2) the age-specific continuation rates for FTE teachers for each year from 2008 through 2020 are similar to either the projections produced using single exponential smoothing or the values for 2008, depending on the age of the teachers (step 2); (3) the age distribution for newly hired FTE teachers from 2008 through 2020 is similar to that of newly hired full-time and part-time teachers in 2007 (step 3); (4) the actual numbers of FTE teachers for each year from 2008 through 2020 are similar to projections of FTE teachers shown in table 16 on page 53; and (5) no economic or political changes further affect the size of the teaching force.

\section{Data used for projections of new teacher hires}

Data on numbers of public school teachers. Numbers of FTE teachers for 2008 came from the NCES Common Core of Data (CCD).

Data on numbers of private school teachers. Private school data on the numbers of FTE teachers in 2003-04, 2005-06, 2007-08, and 2009-10 came from the biennial NCES Private School Universe Survey (PSS). Since the PSS is collected in the fall of odd numbered years, data for years without a PSS collection were estimated using data from the PSS.

Data on the age distribution of public and private school teachers. Data on the age distribution of full-time and part-time public and private school teachers came from the 2007-08 NCES Schools and Staffing Survey (SASS). These data and their standard errors are shown in table A-12 on page 110.

Data on the age distribution of public and private new teacher hires. Data on the age distribution of newly hired full-time and part-time public and private school teachers came from the 2007-08 NCES Schools and Staffing Survey (SASS). These data and their standard errors are shown in table A-13 on page 110.

Data on and projections of age-specific continuation rates of public and private school teachers. The 2008 continuation rates came from the 2008-09 NCES Teacher Follow-Up Survey (TFS). Data from the 1994-95, 2000-01, and 2004-05 TFS were also used in the projection of age-specific continuation rates. The actual data, their standard errors, and the projections are shown in table A-14 on page 111.

Projections of the numbers of public and private elementary and secondary school teachers. These projections are described under "Elementary and Secondary Teacher Model," earlier in this section of appendix A.

\section{Accuracy of projections of new teacher hires}

Because this is the fourth edition of Projections of Education Statistics to include projections of new teacher hires, there are too few years of data to present the MAPEs for new teacher hires. 
Breusch-

Godfrey

Serial

Dependent

Correlation

variable

LM test

Time

Equation ${ }^{1} \quad R^{2} \quad$ statistic $^{2}$

period

\begin{tabular}{|c|c|c|c|c|c|c|c|c|c|}
\hline Elementary & $\ln ($ RELENRTCH) $=$ & $\begin{array}{r}3.81+ \\
(40.307)\end{array}$ & $\begin{array}{c}.07 \ln (\text { RSALARY }) \\
(5.102)\end{array}$ & $\begin{array}{r}.23 \ln (\text { RSGRNTELENR) } \\
(-10.551)\end{array}$ & + & $\begin{array}{r}.32 \mathrm{AR}(1) \\
(1.989)\end{array}$ & 0.99 & $.03(0.985)$ & $\begin{array}{r}1973 \text { to } \\
2009\end{array}$ \\
\hline $\mathrm{Cc}$ & $(\mathrm{RSCENRTCH})=$ & $\begin{array}{r}4.14- \\
(41.407)\end{array}$ & $\begin{array}{r}.24 \ln (\text { RSGRNTSCENR) }+ \\
(-16.565)\end{array}$ & $\begin{array}{r}.49 \ln (\text { RSCENRPU) } \\
(4.293)\end{array}$ & + & $\begin{array}{r}.63 \mathrm{AR}(1) \\
(4.383)\end{array}$ & 0.99 & 12) & 197 \\
\hline
\end{tabular}

${ }^{1} \mathrm{AR}(1)$ indicates that the model was estimated using least squares with the $\mathrm{AR}(1)$ process for correcting for first-order autocorrelation. To estimate the model, it was first transformed into a nonlinear model and then the coefficients were estimated simultaneously by applying a Marquardt nonlinear least squares algorithm to the transformed equation. For a general discussion of the problem of autocorrelation, and the method used to forecast in the presence of autocorrelation, see Judge, G., Hill, W., Griffiths, R., Lutkepohl, H., and Lee, T. (1985). The Theory and Practice of Econometrics, New York: John Wiley and Sons, pp. 315-318.

2 The number in parentheses is the probability of the Chi-Square(2) associated with the Breusch-Godfrey Serial Correlation LM Test. A $p$ value greater than 0.05 implies that we do not reject the null hypothesis of no autocorrelation at the 5 percent significance level for a two-tailed test and the 10 percent significance level for a one-tailed test (i.e., there is no autocorrelation present). For an explanation of the Breusch-Godfrey Serial Correlation LM test statistic, see Greene, W. (2000). Econometric Analysis. New Jersey: Prentice-Hall.

NOTE: $R^{2}$ indicates the coefficient of determination. Numbers in parentheses are $t$-statistics.

RELENRTCH = Ratio of public elementary school enrollment to classroom teachers (i.e., pupil/teacher ratio).

RSCENRTCH = Ratio of public secondary school enrollment to classroom teachers (i.e., pupil/teacher ratio).

RSALARY = Average annual teacher salary relative to the overall economy wage in 2000 dollars.

RSGRNTELENR = Ratio of education revenue receipts from state sources per capita to public elementary school enrollment in 2000 dollars

RSGRNTSCENR = Ratio of education revenue receipts from state sources per capita to public secondary school enrollment in 2000 dollars.

RSCENRPU = Ratio of enrollment in public secondary schools to the 11- to 18-year-old population.

SOURCE: U.S. Department of Education, National Center for Education Statistics, Elementary and Secondary Teacher Model, 1973-2009. (This table was prepared March 2011.) 
Table A-12. Percentage distribution of full-time and part-time school teachers, by age, control of school, and teaching status: 2007-08

\begin{tabular}{|c|c|c|c|c|c|c|c|c|c|c|c|c|c|}
\hline \multirow[b]{2}{*}{$\begin{array}{l}\text { Control of school } \\
\text { and teaching status }\end{array}$} & \multirow[b]{2}{*}{$\begin{array}{l}\text { Percent } \\
\text { of total }\end{array}$} & \multicolumn{12}{|c|}{ Age distribution } \\
\hline & & Total & & $\begin{array}{l}5 \text { s than } \\
5 \text { years }\end{array}$ & $\begin{array}{r}25-29 \\
\text { years }\end{array}$ & & $\begin{array}{r}30-39 \\
\text { years }\end{array}$ & & $\begin{array}{r}40-49 \\
\text { years }\end{array}$ & & $\begin{array}{r}50-59 \\
\text { years }\end{array}$ & $\begin{array}{r}60-64 \\
\text { years }\end{array}$ & $\begin{array}{l}65 \text { years } \\
\text { or more }\end{array}$ \\
\hline \multicolumn{14}{|l|}{ Public-actual } \\
\hline 2007-08 & 100.0 & 100.0 & 3.7 & $(0.21)$ & $14.3(0.51)$ & 26.4 & (0.39) & 23.7 & $(0.47)$ & 25.8 & $(0.51)$ & $4.8(0.24)$ & $1.3(0.12)$ \\
\hline Full-time & $91.8(0.29)$ & 100.0 & 3.8 & $(0.22)$ & $14.6(0.50)$ & 26.5 & $(0.40)$ & 23.6 & $(0.50)$ & 25.7 & $(0.54)$ & $4.7(0.25)$ & $1.2(0.13)$ \\
\hline Part-time & $8.2(0.29)$ & 100.0 & 2.5 & $(0.46)$ & $11.8(1.18)$ & 25.3 & (1.56) & 24.7 & $(1.48)$ & 27.6 & (1.33) & $6.0(0.83)$ & $2.1(0.34)$ \\
\hline \multicolumn{14}{|l|}{ Private-actual } \\
\hline 2007-08 & 100.0 & 100.0 & 4.6 & $(0.34)$ & $11.7(0.48)$ & 22.3 & (0.91) & 23.8 & $(0.65)$ & 26.2 & $(0.87)$ & $7.9(0.52)$ & $3.6(0.41)$ \\
\hline Full-time & $78.8(0.93)$ & 100.0 & 5.0 & (0.37) & $13.0(0.66)$ & 23.0 & (0.96) & 23.0 & $(0.65)$ & 25.0 & $(0.98)$ & $8.0(0.56)$ & $3.0(0.38)$ \\
\hline Part-time & $21.2(0.93)$ & 100.0 & 3.0 & $(0.80)$ & $7.0(0.90)$ & 19.0 & (1.86) & 27.0 & $(1.90)$ & 29.0 & $(1.46)$ & $9.0(1.57)$ & $7.0(1.09)$ \\
\hline
\end{tabular}

Table A-13. Percentage distribution of full-time and part-time newly hired teachers, by age and control of school: Selected years, 1987-88 through 2007-08

\begin{tabular}{|c|c|c|c|c|c|c|c|c|c|c|c|c|c|c|}
\hline \multirow[b]{2}{*}{$\begin{array}{l}\text { Control of school and } \\
\text { school year }\end{array}$} & \multicolumn{14}{|c|}{ Age distribution } \\
\hline & Total & $\begin{array}{r}\text { Les } \\
25\end{array}$ & $\begin{array}{l}\text { ss than } \\
5 \text { years }\end{array}$ & & $\begin{array}{r}25-29 \\
\text { years }\end{array}$ & & $\begin{array}{r}30-39 \\
\text { years }\end{array}$ & & $\begin{array}{r}40-49 \\
\text { years }\end{array}$ & & $\begin{array}{r}50-59 \\
\text { years }\end{array}$ & & $\begin{array}{r}60-64 \\
\text { years }\end{array}$ & $\begin{array}{l}65 \text { years } \\
\text { or more }\end{array}$ \\
\hline \multicolumn{15}{|l|}{ Public } \\
\hline 1987-88 & 100.0 & 17.7 & $(0.79)$ & 23.7 & (1.19) & 33.0 & $(1.43)$ & 21.2 & $(0.80)$ & 4.0 & $(0.51)$ & $0.3 !$ & $(0.11)$ & $(\dagger)$ \\
\hline 1990-91 & 100.0 & 17.5 & $(1.06)$ & 24.0 & (1.35) & 30.6 & (1.33) & 21.4 & (1.28) & 5.6 & $(0.65)$ & 0.6 & $(0.18)$ & $(\dagger)$ \\
\hline 1993-94 & 100.0 & 16.2 & $(0.91)$ & 28.7 & (1.15) & 24.9 & (1.04) & 24.6 & (1.16) & 5.0 & $(0.63)$ & 0.5 & $(0.13)$ & $0.2 !(0.09)$ \\
\hline 1999-2000 & 100.0 & 23.6 & $(1.28)$ & 22.5 & (0.97) & 22.2 & (1.10) & 19.2 & $(0.90)$ & 11.1 & $(0.88)$ & 0.9 & $(0.23)$ & $0.6 !(0.26)$ \\
\hline 2003-04 & 100.0 & 24.4 & $(1.21)$ & 19.0 & (1.23) & 24.6 & (1.10) & 16.5 & (1.18) & 13.3 & $(0.93)$ & 1.5 & $(0.29)$ & $0.7 !(0.29)$ \\
\hline 2007-08 & 100.0 & 23.8 & $(1.75)$ & 24.3 & (1.79) & 20.4 & (1.56) & 15.1 & $(0.94)$ & 13.6 & (1.22) & 2.3 & $(0.39)$ & $0.5 !(0.22)$ \\
\hline \multicolumn{15}{|l|}{ Private } \\
\hline 1987-88 & 100.0 & 17.0 & $(1.27)$ & 22.8 & $(1.68)$ & 32.5 & $(2.17)$ & 17.9 & $(1.61)$ & 5.3 & $(1.09)$ & $\ddagger$ & $(\dagger)$ & $1.8 !(0.77)$ \\
\hline 1990-91 & 100.0 & 15.8 & (1.47) & 26.3 & (1.83) & 29.1 & (1.86) & 21.1 & (1.67) & 5.6 & $(0.88)$ & $1.1 !$ & $(0.40)$ & $1.0 !(0.42)$ \\
\hline 1993-94 & 100.0 & 19.3 & (1.13) & 24.4 & (1.19) & 24.9 & (1.49) & 22.6 & (1.18) & 7.3 & $(0.85)$ & 0.9 & $(0.20)$ & $0.6 !(0.23)$ \\
\hline 1999-2000 & 100.0 & 18.5 & $(0.89)$ & 17.2 & (0.87) & 24.1 & (1.24) & 22.1 & (1.19) & 14.0 & (1.01) & 2.6 & $(0.39)$ & $1.5(0.38)$ \\
\hline 2003-04 & 100.0 & 17.1 & (1.59) & 16.0 & (2.13) & 23.0 & (2.19) & 22.8 & (3.32) & 15.3 & $(1.77)$ & 3.6 & $(0.83)$ & $2.1 \quad(0.58)$ \\
\hline 2007-08 & 100.0 & 14.3 & $(1.26)$ & 18.2 & (1.36) & 23.2 & $(1.97)$ & 23.6 & (1.92) & 14.4 & (1.49) & 4.2 & $(0.84)$ & $2.1 !(0.69)$ \\
\hline
\end{tabular}

† Not applicable.

! Interpret data with caution. The coefficient of variation (CV) for this estimate is 30 percent or greater.

¥ Reporting standards not met. The coefficient of variation (CV) for this estimate is 50 percent or greater.

NOTE: Detail may not sum to totals because of rounding. Standard errors appear in parentheses. The 2007-08 data are the most recent data available.

SOURCE: U.S. Department of Education, National Center for Education Statistics, Schools and Staffing Survey (SASS), "Public School Teacher

Questionnaire," 1987-88 through 2007-08 and "Private School Teacher Questionnaire," 1987-88 through 2007-08; and unpublished tabulations.

(This table was prepared October 2010.) 
Table A-14. Actual and projected continuation rates of full-time and part-time school teachers, by age and control of school: Selected years, 1993-94 to 1994-95 through 2020-21 to 2021-22

\begin{tabular}{|c|c|c|c|c|c|c|c|c|c|c|c|c|c|c|c|c|}
\hline \multirow{2}{*}{$\begin{array}{l}\text { Control of school } \\
\text { and school year }\end{array}$} & \multirow{2}{*}{\multicolumn{2}{|c|}{ Total }} & \multicolumn{14}{|c|}{ Continuation rates, by age } \\
\hline & & & \multicolumn{2}{|c|}{$\begin{array}{l}\text { Less than } \\
25 \text { years }\end{array}$} & \multicolumn{2}{|c|}{$\begin{array}{r}25-29 \\
\text { years }\end{array}$} & \multicolumn{2}{|c|}{$\begin{array}{l}30-39 \\
\text { years }\end{array}$} & \multicolumn{2}{|c|}{$\begin{array}{l}40-49 \\
\text { years }\end{array}$} & \multicolumn{2}{|c|}{$\begin{array}{l}50-59 \\
\text { years }\end{array}$} & \multicolumn{2}{|r|}{$\begin{array}{l}60-64 \\
\text { years }\end{array}$} & \multicolumn{2}{|c|}{$\begin{array}{l}65 \text { years } \\
\text { or older }\end{array}$} \\
\hline \multicolumn{17}{|l|}{ Public actual } \\
\hline $1993-9$ & 3.4 & $(0.36)$ & 96.2( & .09) & 90.0 & 1.22) & 93.3 & (1.03) & 96.1 & $(0.54)$ & 93.7 & $(0.77)$ & 69.5 & $(4.79)$ & 65.9 & $(8.8$ \\
\hline \multicolumn{17}{|l|}{$1999-2000$ to } \\
\hline $03-04$ to $2004-05$ & 91.4 & $(0.55)$ & 4.9 & $.79)$ & 0.1 & 1.71) & 92.6( & $(0.93)$ & 94.5 & (0.78) & 90.8 & $(0.81)$ & 77.2 & $(3.00)$ & 70.3 & $(9.40$ \\
\hline $2007-08$ to & 1.8 & $(0.45)$ & 92.2( & $.95)$ & 9.0 & 2.33) & 92.4 & (1.29) & 95.1 & (1.06) & 92.3 & (1.23) & 82.8 & (3.97) & 88.9 & (4.26) \\
\hline \multicolumn{17}{|l|}{ Public projected } \\
\hline $2008-09$ to $2009-10$ & .7 & $(\dagger)$ & 88.9 & $(\dagger)$ & 89.0 & $(†)$ & 92.6 & $(\dagger)$ & 94.1 & $(\dagger)$ & 91.4 & $(\dagger)$ & 76.5 & $(\dagger)$ & 65.0 & \\
\hline 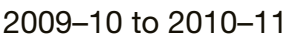 & .6 & $(\dagger)$ & 89.2 & $(\dagger)$ & 89.0 & $(\dagger)$ & 92.6 & $(†)$ & 93.9 & $(\dagger)$ & 91.4 & $(\dagger)$ & & $(\dagger)$ & 64.6 & \\
\hline & & $(†)$ & 88.7 & $(\dagger)$ & 89.0 & $(†)$ & 92.6 & $(†)$ & 94.0 & $(†)$ & & $(\dagger)$ & & $(\dagger)$ & 63.7 & \\
\hline & & $(\dagger)$ & 89.0 & $(\dagger)$ & & $(†)$ & 92.6 & $(\dagger)$ & & $(\dagger)$ & & $(\dagger)$ & & $(\dagger)$ & 2.6 & \\
\hline & & $(\dagger)$ & 88.9 & $(\dagger)$ & 89.0 & $(†)$ & 92.6 & $(\dagger)$ & 92 & $(\dagger)$ & 91.4 & $(\dagger)$ & & †) & 63.3 & \\
\hline & & $(\dagger)$ & 88.9 & $(\dagger)$ & 89.0 & $(\dagger)$ & 92.6 & $(\dagger)$ & 94.1 & $(\dagger)$ & 1.5 & $(\dagger)$ & 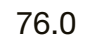 & $(\dagger)$ & 63.1 & \\
\hline & 5 & $(†)$ & 88.8 & $(\dagger)$ & 89.0 & (†) & 92.6 & $(†)$ & 94.0 & $(†)$ & 1.5 & $(†)$ & & $(†)$ & 4.4 & \\
\hline & & $(\dagger)$ & 88.9 & $(\dagger)$ & 89.0 & (†) & 92.6 & $(\dagger)$ & & $(\dagger)$ & & $(\dagger)$ & & $(\dagger)$ & 4.1 & \\
\hline & & $(\dagger)$ & 88.9 & $(\dagger)$ & & $(†)$ & & $(\dagger)$ & & f) & & $(\dagger)$ & & †) & 3.8 & \\
\hline & & $(†)$ & 89.0 & $(\dagger)$ & 89.0 & (†) & 92.6 & $(†)$ & 94 & $(†)$ & 91.6 & $(†)$ & & $(†)$ & 3.8 & \\
\hline & & $(†)$ & 89.0 & $(\dagger)$ & 89.0 & (†) & 92.6 & $(\dagger)$ & 94.1 & †) & 1. & $(\dagger)$ & 75 & †) & 3.9 & \\
\hline & & $(\dagger)$ & 88.9 & $(\dagger)$ & 890 & $(†)$ & 92.6 & $(\dagger)$ & 94 & )) & 1 & $(\dagger)$ & 75 & $(\dagger)$ & 63.7 & \\
\hline $2020-$ & & $(†)$ & 89.0 & $(\dagger)$ & 89.0 & (†) & 92.5 & $(†)$ & 94.0 & $(†)$ & 91.6 & $(†)$ & 76.0 & $(†)$ & 63.4 & \\
\hline \multicolumn{17}{|l|}{ Private } \\
\hline 1993 & t & 74) & \multirow{2}{*}{\multicolumn{2}{|c|}{$80.0(4.42)$}} & \multirow{2}{*}{\multicolumn{2}{|c|}{86.9 (1.64) }} & \multirow{2}{*}{\multicolumn{2}{|c|}{$85.1(1.70)$}} & \multirow{2}{*}{\multicolumn{2}{|c|}{$91.3(1.14)$}} & \multicolumn{2}{|c|}{$91.8(1.52)$} & 86.9 & 4) & \multicolumn{2}{|c|}{58.1 (8.67) } \\
\hline $1999-2000$ to & & & & & & & & & & & & & & & & \\
\hline 2000-01 & & 72) & 1.7 & & 72.2 & & 80.2 & 57) & 86 & & 2.3 & & 8.8 & .79) & 75.2 & $(5.1$ \\
\hline 2003- & & $(2.06)$ & 5.4 & & 71.7 & 3.62) & 82.2 & (2.30) & 86.8 & (2.28) & 9.2 & (9.17) & 0.1 & 4.15) & 79.5 & $(6.07$ \\
\hline $2007-08$ to 200 & .2 & (1.69) & 77.7 & $33)$ & 71.7 & $6.44)$ & 79.1 & $(3.43)$ & 86.1 & (2.92) & 86.8 & (2.17) & 85.2 & $(4.21)$ & 77.3 & $(8.2$ \\
\hline & & & & & & & & & & & & & & & & \\
\hline & & (†) & 685 & $(\dagger)$ & 731 & (†) & 80.5 & $(†)$ & 86.2 & $(†)$ & 87.7 & $(†)$ & 80 & $(†)$ & 78.4 & \\
\hline & & $(†)$ & 68.2 & $(\dagger)$ & 73.2 & (†) & 80.5 & $(†)$ & 86.2 & )) & 87 & $(†)$ & 7 & $(†)$ & 74.6 & \\
\hline & & $(\dagger)$ & 68.1 & $(\dagger)$ & 73.4 & $(\dagger)$ & 80.4 & $(\dagger)$ & 86.1 & $(\dagger)$ & 87.8 & $(\dagger)$ & & $(\dagger)$ & 76.7 & \\
\hline & & (†) & 67.8 & $(\dagger)$ & 73.3 & (†) & 80.3 & $(†)$ & 86.2 & $(†)$ & 87.8 & (†) & 1 & $(†)$ & 75.0 & \\
\hline & & $(†)$ & 68.0 & & 73.2 & (†) & 80.3 & $(†)$ & 86.1 & & 87.7 & & & †) & 75.3 & \\
\hline & & $(\dagger)$ & 68.0 & $(\dagger)$ & 73 & $(†)$ & 80. & $(†)$ & 86 & & 87 & & 80 & 7 & 76.9 & \\
\hline & & $(\dagger$ & 68.0 & ( & 73.1 & (†) & 80 & $+(+)$ & 86 & & 87 & & 70 & $f$ & 76.4 & \\
\hline 6 to 2 & & $(\dagger)$ & 67.9 & $(\mathrm{~T})$ & 73.1 & (†) & 80.3 & $(†)$ & 86.3 & $(\dagger)$ & 87.6 & $(\dagger)$ & 8 & $(\dagger)$ & 75.7 & \\
\hline $2016-17$ to $2017-1$ & 2.2 & $(†)$ & 67.9 & $(\dagger)$ & 73.1 & (†) & 80.4 & $(†)$ & 86.2 & $(†)$ & 87.8 & $(†)$ & 80.4 & $(†)$ & 76.2 & \\
\hline 201 & O & $(\dagger)$ & 67.9 & $(\dagger)$ & 73.1 & $(†)$ & 80.3 & $(†)$ & 86.3 & $(†)$ & 87.8 & $(\dagger)$ & 80.2 & $(\dagger)$ & 76.0 & \\
\hline & 6.2 & $(\dagger)$ & 67.9 & $(\dagger)$ & 73.1 & $(†)$ & 80.3 & $(\dagger)$ & 86.3 & & 87.7 & $(\dagger)$ & 80 & $(\dagger)$ & 75.6 & \\
\hline & & $(†)$ & 67.9 & $(\dagger)$ & 73.1 & $(†)$ & 80.3 & $(†)$ & 86.3 & $(\dagger)$ & 87.7 & $(†)$ & 80 & $(†)$ & 76.0 & \\
\hline $2020-21$ to $2021-22$ & 82.1 & $(\dagger)$ & 67.9 & $(\dagger)$ & 73.1 & $(†)$ & 80.3 & $(\dagger)$ & 86.3 & $(\dagger)$ & 87.7 & $(†)$ & 80.1 & $(†)$ & 76.1 & \\
\hline
\end{tabular}

† Not applicable.

! Interpret with caution. The coefficient of variation (CV) for this estimate is 30 percent or greater.

$\ddagger$ Reporting standards not met. The coefficient of variation (CV) for this estimate is 50 percent or greater.

NOTE: The continuation rate for teachers for each control of school (public schools and private schools) is the percentage of teachers in that control who continued teaching in the same control from one year to the next. Standard errors appear in parentheses.

SOURCE: U.S. Department of Education, National Center for Education Statistics, Teacher Follow up Survey (TFS), "Public School Teacher Questionnaire," 1994-95 through 2008-09 and "Private School Teacher Questionnaire," 1994-95 through 2008-09; and unpublished tabulations.

(This table was prepared January 2011.) 


\section{A.4. EXPENDITURES FOR PUBLIC ELEMENTARY AND SECONDARY EDUCATION}

\section{Projections in this edition}

This edition of Projections of Education Statistics presents projections of total current expenditures for public elementary and secondary education, current expenditures per pupil in fall enrollment, and current expenditures per pupil in average daily attendance for 2008-09 through 2020-21.

As the source of the elementary and secondary private school data, the NCES Private School Universe Survey, does not collect data for current expenditures, there are no projections for private school current expenditures.

\section{Overview of approach}

\section{Theoretical and empirical background}

The Public Elementary and Secondary Education Current Expenditure Model used in this report is based on the theoretical and empirical literature on the demand for local public services such as education. ${ }^{1}$ Specifically, it is based on a type of model that has been called a median voter model. In brief, a median voter model posits that spending for each public good in the community (in this case, spending for education) reflects the preferences of the "median voter" in the community. This individual is identified as the voter in the community with the median income and median property value. The amount of spending in the community reflects the price of education facing the voter with the median income, as well as his income and tastes. There are competing models in which the level of spending reflects the choices of others in the community, such as government officials.

In a median voter model, the demand for education expenditures is typically linked to four different types of independent variables: (1) measures of the income of the median voter; (2) measures of intergovernmental aid for education going indirectly to the median voter; (3) measures of the price to the median voter of providing one more dollar of education expenditures per pupil; and (4) any other variables that may affect one's tastes for education. The Public Elementary and Secondary Education Current Expenditure Model contains independent variables of the first two types. It uses multiple linear regression analysis to define the relationships between these independent variables and current expenditures (the dependent variable).

\section{Elementary and Secondary Education Current Expenditure Model}

Projections for current expenditures per pupil in fall enrollment were produced first. These projections were then used in calculating total expenditures and expenditures per pupil in average daily attendance.

\section{Steps used to project current expenditures for public elementary and secondary education}

Step 1. Produce projections of education revenue from state sources. The equation for education revenue included an $A R(1)$ term for correcting for autocorrelation and the following independent variables:

» disposable income per capita in constant dollars; and

$»$ the ratio of fall enrollment to the population.

To estimate the model, it was first transformed into a nonlinear model and then the coefficients were estimated simultaneously by applying a Marquardt nonlinear least squares algorithm to the transformed equation.

Step 2. Produce projections of current expenditures per pupil in fall enrollment. The equation for current expenditures per pupil for fall enrollment included an AR(1) term for correcting for autocorrelation and the following independent variables:

$»$ disposable income per capita in constant dollars; and

» education revenue from state sources per capita in constant dollars. This variable was projected in step 1.

\footnotetext{
${ }^{1}$ For a discussion of the theory together with a review of some of the older literature, see Inman (1979). More recent empirical work includes Gamkhar and Oates (1996) and Mitias and Turnbull (2001).
} 
To estimate the models, they were first transformed into nonlinear models and then the coefficients were estimated simultaneously by applying a Marquardt nonlinear least squares algorithm to the transformed equation.

For details on the equations used in steps 1 and 2, the data used to estimate these equations, and their results, see "Data and equations used for projections of current expenditures for public elementary and secondary education," below.

Step 3. Produce projections of total current expenditures. Projections of total current expenditures were made by multiplying the projections for current expenditures per pupil in fall enrollment by projections for fall enrollment.

Step 4. Produce projections of current expenditures per pupil in average daily attendance. The projections for total current expenditures were divided by projections for average daily attendance to produce projections of current expenditures per pupil in average daily attendance.

All the projections were developed in 1982-84 dollars and then placed in 2008-09 dollars using the projections of the Consumer Price Index. Current-dollar projections were produced by multiplying the constant-dollar projections by projections for the Consumer Price Index. The Consumer Price Index and the other economic variables used in calculating the projections presented in this report were placed in school year terms rather than calendar year terms.

\section{Data and equations used for projections of current expenditures for public elementary and secondary education}

Data used to estimate the equations for revenue from state sources and current expenditures per pupil. The following data for the period from 1973-74 to 2007-08 were used to estimate the equations:

»Current expenditures and revenues from state sources—For 1973-74 and 1975-76, the current expenditures data came from Statistics of State School Systems, published by NCES. For 1974-75 and 1976-77, the current expenditures data came from Revenues and Expenditures for Public Elementary and Secondary Education, also published by NCES. For 1977-78 through 2007-08, these data came from the NCES Common Core of Data (CCD) and unpublished data. For most years, the sources for the past values of revenue from state sources were identical to the sources for current expenditures.

»Disposable personal income per capita-Disposable personal income from the Bureau of Economic Analysis were divided by population data from the U.S. Census Bureau.

» The ratio of fall enrollment to population data-Fall enrollment data from the CCD were divided by population data from the U.S. Census Bureau.

Estimated equations and model statistics for revenue from state sources and current expenditures per pupil. For the results of the equations, see table A-15 on page 115. In each equation, the independent variables affect the dependent variable in the expected way. In the revenues from state sources equation:

»All other things being equal, as disposable income per capita increases so does local governments' education revenue from state sources per capita; and

»As enrollment increases relative to the population, so does the local governments' education revenue from state sources per capita.

»In the current expenditures per pupil equation: All other things being equal, as disposable income per capita increases, so does current expenditures per pupil; and

»As local governments' education revenue from state sources per capita increases, so does current expenditures per pupil.

Projections for economic variables. Projections for economic variables, including disposable income and the Consumer Price Index, were from the "U.S. Monthly Model: November 2009 Short-Term Projections" from the economic consulting firm, IHS Global Insight (see supplemental table B-6). The values of all the variables from IHS Global Insight were placed in school-year terms. The school-year numbers were calculated by taking the average of the last two quarters of one year and the first two quarters of the next year.

Projections for fall enrollment. The projections for fall enrollment are those presented in section 1 of this publication. The methodology for these projections is presented in Section A.1. Elementary and Secondary Enrollment, earlier in this appendix.

Projections for population. Population estimates for 1973 to 2009 and population projections for 2010 to 2020 from the U.S. Census Bureau were used to develop the public school current expenditure projections. The set of population projections used in this year's Projections of Education Statistics are the Census Bureau's 2008 National Population Projections (August 2008). 
Historical data for average daily attendance. For 1973-74 and 1975-76, these data came from Statistics of State School Systems, published by NCES. For 1974-75 and 1976-77, the current expenditures data came from Revenues and Expenditures for Public Elementary and Secondary Education, also published by NCES. For 1977-78 through 2007-08, these data came from the CCD and unpublished NCES data.

Projections for average daily attendance. These projections were made by multiplying the projections for enrollment by the average value of the ratios of average daily attendance to enrollment from 1993-94 to 2007-08; this average value was approximately 0.94 .

\section{Accuracy of projections}

Mean absolute percentage errors (MAPEs) for projections of current expenditures for public elementary and secondary education were calculated using the last 20 editions of Projections of Education Statistics. Table D, below, shows the MAPEs for projections of current expenditures.

Table D. Mean absolute percentage errors (MAPEs) of projections for total and per pupil current expenditures for public elementary and secondary education, by lead time: 2011

\begin{tabular}{|c|c|c|c|c|c|c|c|c|c|c|}
\hline \multirow[b]{2}{*}{ Statistic } & \multicolumn{10}{|c|}{ Lead time (years) } \\
\hline & 1 & 2 & 3 & 4 & 5 & 6 & 7 & 8 & 9 & 10 \\
\hline Total current expenditures & 1.3 & 2.0 & 2.1 & 2.2 & 2.6 & 3.3 & 4.1 & 4.3 & 4.0 & 4.1 \\
\hline Current expenditures per pupil in fall enrollment & 1.3 & 2.0 & 2.0 & 2.1 & 3.0 & 3.6 & 4.4 & 4.9 & 5.2 & 5.5 \\
\hline
\end{tabular}

NOTE: Expenditures were in constant dollars based on the Consumer Price Index for all urban consumers, Bureau of Labor Statistics, U.S. Department of Labor. MAPEs for current expenditures were calculated using projections from the last 20 editions of Projections of Education Statistics containing current expenditure projections. Calculations were made using unrounded numbers. Some data have been revised from previously published figures.

SOURCE: U.S. Department of Education, National Center for Education Statistics, Projections of Education Statistics, various issues. (This table was prepared March 2011.)

For more information about MAPEs, see Section A.O. Introduction, earlier in this appendix. 
Table A-15. Estimated equations and model statistics for current expenditures per pupil in fall enrollment for public elementary and secondary schools, and education revenue from state sources per capita

\begin{tabular}{|c|c|c|c|c|c|c|c|}
\hline Dependent variable & & & & Equation $^{1}$ & $R^{2}$ & $\begin{array}{r}\text { Breusch- } \\
\text { Godfrey } \\
\text { Serial } \\
\text { Correlation } \\
\text { LM test } \\
\text { statistic }^{2}\end{array}$ & $\begin{array}{r}\text { Time } \\
\text { period }\end{array}$ \\
\hline $\begin{array}{l}\text { Current expenditures } \\
\text { per pupil }\end{array}$ & $\begin{array}{l}0.58+ \\
(0.371)\end{array}$ & $\begin{array}{c}0.64 \ln (\mathrm{PCl}) \\
(3.814)\end{array}$ & $\begin{array}{c}0.21 \ln (\text { SGRANT) } \\
(2.456)\end{array}$ & $\begin{array}{r}0.93 A R(1) \\
(18.176)\end{array}$ & 0.997 & $2.42(0.30)$ & $\begin{array}{r}1973-74 \text { to } \\
2007-08\end{array}$ \\
\hline $\begin{array}{l}\text { Education revenue from } \\
\text { state sources per capita } \ln (\mathrm{SGRANT})=\end{array}$ & $\begin{array}{c}0.85 \\
(0.724)+\end{array}$ & $\begin{array}{l}1.12 \ln (\mathrm{PCl}) \\
(20.324)+\end{array}$ & $\begin{array}{r}0.73 \ln (\text { ENRPOP) } \\
(4.350)+\end{array}$ & $\begin{array}{r}0.54 \mathrm{AR}(1) \\
(4.153)\end{array}$ & 0.988 & $2.31(0.31)$ & $\begin{array}{r}1973-74 \text { to } \\
2007-08\end{array}$ \\
\hline
\end{tabular}

${ }^{1} A R(1)$ indicates that the model was estimated using least squares with the AR(1) process for correcting for first-order autocorrelation. To estimate the model, it was first transformed into a nonlinear model and then the coefficients were estimated simultaneously by applying a Marquardt nonlinear least squares algorithm to the transformed equation. For a general discussion of the problem of autocorrelation, and the method used to forecast in the presence of autocorrelation, see Judge, G., Hill, W., Griffiths, R., Lutkepohl, H., and Lee, T. The Theory and Practice of Econometrics, New York: John Wiley and Sons, 1985, pp. 315-318.

2 The number in parentheses is the probability of the Chi-Square(2) associated with the Breusch-Godfrey Serial Correlation LM Test. A $p$ value greater than 0.05 implies that we do not reject the null hypothesis of no autocorrelation at the 5 percent significance level for a two-tailed test and the 10 percent significance level for a one-tailed test (i.e., there is no autocorrelation present). For an explanation of the Breusch-Godfrey Serial Correlation LM test statistic, see Greene, W. Econometric Analysis. New Jersey: Prentice-Hall, 2000.

NOTE: $R^{2}$ indicates the coefficient of determination. Numbers in parentheses are $t$-statistics.

CUREXP = Current expenditures of public elementary and secondary schools per pupil in fall enrollment in constant 1982-84 dollars.

SGRANT = Local governments' education revenue from state sources, per capita, in constant 1982-84 dollars.

$\mathrm{PCl}=$ Disposable income per capita in constant 2000 chained dollars.

$\mathrm{ENRPOP}=$ Ratio of fall enrollment to the population.

SOURCE: U.S. Department of Education, National Center for Education Statistics, Elementary and Secondary School Current Expenditures Model, 1973-74 through 2007-08; and Revenue Receipts from State Sources Model, 1973-74 through 2007-08. (This table was prepared March 2011.) 


\section{A.5. ENROLLMENT IN POSTSECONDARY DEGREE-GRANTING INSTITUTIONS}

\section{Projections in this edition}

This edition of Projections of Education Statistics presents projections of enrollment in postsecondary degree-granting institutions for fall 2010 through fall 2020. Three different models were used to produce these enrollment projections:

» The Enrollment in Degree-Granting Institutions Model produced projections of enrollments by attendance status, level of student, level of institution, control of institution, sex, and age. It also produced projections of full-time-equivalent enrollments by level of student, level of institution, and control of institution.

» The Enrollment in Degree-Granting Institutions by Race/Ethnicity Model produced projections of enrollments by race/ ethnicity.

»The First-Time Freshmen Model produced projections of enrollments of first-time freshmen by sex.

\section{Overview of approach}

\section{Basic features of the three degree-granting enrollment models}

The Enrollment in Degree-Granting Institutions Model is the primary model for projecting enrollment in postsecondary degree-granting institutions. For this model, enrollment rates by attendance status and sex are projected for various age categories using either the pooled seemingly unrelated regression method or the pooled seemingly unrelated regression method with a first-order autocorrelation correction. These rates are applied to projections of populations of the same sex and age to produce projections of enrollment by attendance status, sex, and age. To project enrollments by level of student, level of institution, and control of institution, rates for these characteristics are projected using single exponential smoothing and applied to enrollment projections previously produced by the model.

The Enrollment in Degree-Granting Institutions by Race/Ethnicity Model takes an approach similar to that of the Enrollment in Degree-Granting Institutions Model. Enrollment rates by attendance status, sex, and race/ethnicity are projected for the age categories using either the pooled seemingly unrelated regression method or the pooled seemingly unrelated regression method with a first-order autocorrelation correction. The resulting rates are iteratively corrected to ensure consistency with those projected by the Enrollment in Degree-Granting Institutions Model. The adjusted rates are then applied to projections of populations of the same sex, age, and race/ethnicity.

The First-Time Freshmen Enrollment in Degree-Granting Institutions Model uses single exponential smoothing to project the ratio of freshmen enrollment to undergraduate enrollment separately for males and for females. It then applies the projected ratios to the projections of undergraduate enrollment by sex that were produced by the Enrollment in DegreeGranting Institutions Model.

\section{The Enrollment in Degree-Granting Institutions Model}

The Enrollment in Degree-Granting Institutions Model produces projections of enrollment counts by six levels of detail, as well as projections of full-time-equivalent enrollments by level of student, level of institution, and control of institution.

\section{Steps used in the Enrollment in Degree-Granting Institutions Model}

Step 1. Adjust age-specific enrollment counts from the U.S. Census Bureau to make them agree with the more highly aggregated NCES enrollment counts that do not include age. The Enrollment in Degree-Granting Institutions Model projects enrollments by six levels of detail: attendance status, level of student, level of institution, control of institution, sex, and age. While NCES does produce enrollment counts by the first five levels of detail, it does not produce data by the sixth level of detail, age, every year. However, the U.S. Census Bureau does produce age-specific enrollment counts.

In step 1, the age distributions from the Census Bureau counts for 1980 to 2009 were applied to the NCES counts to produce a set of enrollment data that breaks enrollments down by age while being consistent with NCES counts. Specifically, the most detailed level of Census Bureau data (by attendance status, level of student, level of institution, control of institution, sex, and age) was iteratively changed using proportions based on the more highly aggregated NCES enrollment numbers to ensure that all sums across this most detailed level of Census enrollment data equaled the more highly aggregated NCES enrollment totals that did not include age. 
Step 2. Calculate enrollment rates by attendance status, sex, and age category. The enrollment data were broken up into 14 age categories, with separate age categories for individual ages 14 through 24 as well as for the age groups 25 to 29,30 to 34 , and 35 and over. For each of the 14 age categories, 4 enrollment rates were calculated-part-time male, full-time male, part-time female, and full-time female-resulting in a total of 56 enrollment rates. Each of the 56 enrollment rates was calculated by dividing the enrollment count for that combination of attendance status, sex, and age category by the total population for the corresponding combination of sex and age category. For each combination of attendance and sex, the enrollment rate for the oldest age category was calculated by dividing the enrollment count for those 35 and over by the total population for those 35 to 44 .

Step 3. Produce projections of enrollment rates by attendance status, sex, and age category. Enrollment rates for most of the age groups were projected using multiple linear regression. However, because enrollment in postsecondary degree-granting institutions is negligible for ages 14,15 , and 16, these ages were not included in the multiple linear regression models. Instead, projections for individual ages 14,15 , and 16 were produced by double exponential smoothing.

The following 11 age categories were modeled: individual ages 17 through 24 and age groups 25 to 29, 30 to 34 , and 35 and over. For each of these age categories, enrollment rates by attendance status and sex were produced using four pooled time-series models - one for each combination of attendance status and sex. Each model was pooled across age categories. Each equation contained two independent variables, which were measures of

» disposable income; and

» the unemployment rate.

Either the pooled seemingly unrelated regression method or the pooled seemingly unrelated regression method with a firstorder autocorrelation correction was used to estimate each equation.

For more details on the equations used in step 3, the data used to estimate these equations, and their results, see tables A-16 through A-18 on pages $122-124$.

Step 4. Produce projections of enrollments by attendance status, sex, and age category. For each combination of attendance status, sex, and age category, enrollment projections were produced by multiplying the projected enrollment rate for that combination by projections of the total population with the corresponding combination of sex and age category.

Step 5. Add two additional levels of detail —level of student and level of institution — to the projected enrollments by attendance status, sex, and age category. For this step, the 14 age categories used in the previous steps were collapsed into the following 8 categories: ages 14 to 16, 17, 18 and 19, 20 and 21, 22 to 24, 25 to 29, 30 to 34, and 35 and over. Step 5 can be broken into three parts:

First, the historic data were used to calculate the percentage distribution of enrollment by level of student and level of institution for each combination of attendance status, sex, and age category. Because it was assumed that there was no enrollment in 2-year institutions at the postbaccalaureate level, three combinations of student level and institution type were used: undergraduates at 4-year institutions, undergraduates at 2-year institutions, and postbaccalaureate students at 4-year institutions.

Second, for each combination of attendance status, sex, and age category, the percentage distribution by level of student and level of institution was projected using single exponential smoothing. A separate smoothing constant, chosen to minimize the sum of squared forecast errors, was used in each case. The percentages were then adjusted so the sum of the categories by attendance status, level of student, level of institution, sex, and age category would equal 100 percent.

For the projected percentage distributions from step 5 and the actual 2009 distributions, see tables A-19 and A-20 on pages 125-126.

Third, the projected distributions by level of student and type of institution were applied to the projected enrollments by attendance status, sex, and age category from step 4 to obtain the enrollment projections by attendance status, level of student, level of institution, sex, and age category.

Step 6. Add the sixth level of detail — control of institutions — to the projected enrollments in postsecondary degree-granting institutions. In this step, the data on enrollment by age category were not used. Control of institutions was added in the following manner:

First, the historic data were used to calculate the percentage of enrollment in public institutions for each combination of attendance status, level of student, level of institution, and sex.

Second, the percentages of enrollment in public institutions were projected using single exponential smoothing. A separate smoothing constant, chosen to minimize the sum of squared forecast errors, was used for each percentage. 
For the projected percentages from step 6 and the actual 2009 percentages, see table A-21 on page 126.

Third, the projected percentages were applied to the projected enrollments in each corresponding enrollment combination to obtain projections for public institutions by attendance status, level of student, level of institution, and sex.

Fourth, the projected enrollments for public institutions were subtracted from the total to produce the projected enrollments for private institutions.

Step 7. Produce projections of full-time-equivalent enrollment by level of student, level of institution, and control of institution. Full-time-equivalent enrollment represents total full-time and part-time enrollment as if it were enrollment on a full-time basis. It equals the sum of full-time enrollment plus the full-time-equivalent of part-time enrollment. Full-time-equivalent enrollment projections were produced in the following manner:

First, for each combination of level of student, level of institution, and control of institution, the historic data were used to calculate the full-time-equivalent of part-time enrollment as a percentage of part-time enrollment.

Second, for each combination of level of student, level of institution, and control of institution, the full-time equivalent of part-time enrollment as a percentage of part-time enrollment was projected using single exponential smoothing. A separate smoothing constant, chosen to minimize the sum of squared forecast errors, was used for each percentage.

Third, for each combination of level of student, level of institution, and control of institution, the projected percentages were applied to the projections of part-time enrollment to project the full-time equivalent of the part-time enrollment.

Fourth, the projections of full-time equivalents of part-time enrollment were added to projections of full-time enrollment to obtain projections of full-time-equivalent enrollment.

\section{Data and equation results for the Enrollment in Degree-Granting Institutions Model}

Enrollment data for postsecondary degree-granting institutions. Enrollment data for 1981 to 2009 by attendance status, level of student, level of institution, control of institution, and sex came from the NCES Integrated Postsecondary Education Data System (IPEDS). These are universe counts. The U.S. Census Bureau was the source for enrollment estimates for 1981 to 2009 by the characteristics listed above, as well as age of student.

Population data and projections. Population counts for 1980 to 2009 came from the U.S. Census Bureau. Population projections for 2010 to 2020 are the Census Bureau's 2008 National Population Projections of the population by sex and age (August 2008), ratio-adjusted to line up with the most recent historical estimates. For more information, see Section A.0. Introduction, earlier in this appendix.

Projections for economic variables. The economic variables used in developing these projections were from the "U.S. Monthly Model: November 2010 Short-Term Projections" from the economic consulting firm, IHS Global Insight.

Data and results for the equations. The following details for the equations are shown on pages 122-126:

» Table A-16 shows enrollment rates by sex, attendance status, and age for fall 2009 and projected enrollment rates for fall 2015 and fall 2020.

» Table A-17 shows the estimated equations and model statistics used to project enrollments for men by attendance status, and table A-18 shows the estimated equations and model statistics used to project enrollment rates for women by attendance status. The particular equations shown were selected on the basis of their statistical properties, such as coefficients of determination $\left(\mathrm{R}^{2} s\right)$, the $t$-statistics of the coefficients, the Durbin-Watson statistic, the Breusch-Godfrey Serial Correlation LM test statistic, and residual plots.

» Table A-19 shows actual and projected percentage distributions of full-time students, and table A-20 shows actual and projected percentage distributions of part-time students.

» Table A-21 shows actual and projected data for enrollment in public degree-granting institutions as a percentage of total enrollment by sex, attendance status, level enrolled, and type of institution. 


\section{Accuracy of projections for the Enrollment in Degree-Granting Institutions Model}

Mean absolute percentage errors (MAPEs) for enrollment in degree-granting institutions were calculated using the last 13 editions of Projections of Education Statistics. Table E, below, shows MAPEs for key projections of the Enrollment in DegreeGranting Institutions Model.

Table E. Mean absolute percentage errors (MAPEs) of projected enrollment in postsecondary degree-granting institutions, by lead time, sex, and level of institution: 2011

\begin{tabular}{|c|c|c|c|c|c|c|c|c|c|c|}
\hline \multirow[b]{2}{*}{ Statistic } & \multicolumn{10}{|c|}{ Lead time (years) } \\
\hline & 1 & 2 & 3 & 4 & 5 & 6 & 7 & 8 & 9 & 10 \\
\hline Total enrollment & 1.6 & 2.8 & 3.5 & 4.0 & 5.2 & 6.1 & 8.1 & 9.8 & 11.7 & 11.4 \\
\hline Men & 1.6 & 3.2 & 4.0 & 4.8 & 5.9 & 6.6 & 8.5 & 9.8 & 11.7 & 11.7 \\
\hline Women & 1.8 & 2.8 & 3.6 & 3.7 & 4.6 & 5.8 & 7.8 & 9.8 & 11.7 & 11.2 \\
\hline 4-year institutions & 1.7 & 2.9 & 3.9 & 4.7 & 5.9 & 6.8 & 9.0 & 10.9 & 13.1 & 13.2 \\
\hline 2-year institutions & 2.2 & 3.7 & 4.3 & 4.4 & 5.1 & 5.0 & 6.4 & 7.8 & 9.4 & 8.3 \\
\hline
\end{tabular}

NOTE: MAPEs for degree-granting institution enrollments were calculated using the last 13 editions of Projections of Education Statistics. Calculations were made using unrounded numbers. Some data have been revised from previously published figures.

SOURCE: U.S. Department of Education, National Center for Education Statistics, Projections of Education Statistics, various issues. (This table was prepared March 2011.)

For more information about MAPEs, see Section A.O. Introduction, earlier in this appendix.

\section{The Enrollment in Degree-Granting Institutions by Race/Ethnicity Model}

The Enrollment in Degree-Granting Institutions by Race/Ethnicity Model projects enrollments in degree-granting institutions by attendance status, sex, age, and race/ethnicity. The following groups are projected in this model:

» White;

$»$ Black;

») Hispanic;

»Asian/Pacific Islander;

»American Indian/Alaska Native; and

»nonresident alien.

See the Glossary for definitions of the five racial/ethnic categories and the nonresident alien category. (The race/ethnicity of nonresident aliens is unknown, but they are considered a separate group for purposes of this analysis.)

\section{Steps used in the Degree-Granting Institutions by Race/Ethnicity Model}

Step 1. Adjust U.S. Census Bureau enrollment counts by attendance status, sex, age, and racelethnicity to make them sum to NCES enrollment counts by attendance status, sex, and racelethnicity. For 1981 to 2009, the most detailed levels of Census Bureau enrollment data (by enrollment status, sex, age, and race/ethnicity) were iteratively changed using proportions that were based on the more highly aggregated NCES enrollment numbers to ensure that the sums across these most detailed levels of enrollment data equaled the more highly aggregated NCES enrollment numbers that did not include age.

Step 2. Calculate enrollment rates by attendance status, sex, age category, and racelethnicity. The enrollment data were broken up into 14 age categories, with separate age categories for individual ages 14 through 24 as well as for the age groups 25 to 29,30 to 34 , and 35 and over. For each of the 14 age categories, enrollment rates were calculated for each combination of attendance status, sex, and the six racial/ethnic groups, resulting in a total of 336 enrollment rates. Each of the 336 enrollment rates was calculated by dividing the enrollment count for that combination of attendance status, sex, age category, and race/ethnicity by the total population for the corresponding combination of sex, age category, and race/ ethnicity. For each combination of attendance status, sex and racial/ethnic group, the enrollment rate for the oldest age category was calculated by dividing the enrollment count for those 35 and over by the total population for those 35 to 44 . 
Step 3. Produce projections of enrollment rates by attendance status, sex, age category, and racelethnicity. Enrollment rates for most of the age groups and racial/ethnic groups were projected using multiple linear regression. However, there were several exceptions:

»D Due to negligible enrollments for ages 14,15 , and 16, these ages were not included in the multiple linear regression models. Instead, projections for individual ages 14, 15, and 16 were produced by single exponential smoothing.

»Due to the relatively large fluctuations in the historical enrollment rates resulting from small sample sizes, American Indian/Alaska Native enrollments were projected using single exponential smoothing.

»Since there were no applicable population counts to compute enrollment rates for non-resident aliens, their enrollments were projected using patterns in recent historical growth.

Four racial/ethnic groups were modeled: White, Black, Hispanic, and Asian/Pacific Islander. Eleven age categories were modeled: individual ages 17 through 24 and age groups 25 to 29, 30 to 34, and 35 to 44. For each of the age categories, projected enrollment rates by attendance status, sex, and race/ethnicity were produced using 16 pooled time-series models - one for each combination of attendance status, sex, and the four racial/ethnic groups. Each equation included variables measuring

» recent trends;

$»$ economic conditions (such as disposable income); and

》demographic changes.

For more information on the equations used to project enrollment rates for the combinations of attendance status, sex, and racel ethnicity, see tables A-22 through A-29, under "Data and equations used for the Enrollment in Degree-Granting Institutions by Race/Ethnicity Model," below.

The final set of projected rates by attendance status, sex, age, and race/ethnicity were controlled to enrollment rates by attendance status, sex, and age produced by the Enrollment in Degree-Granting Institutions Model to ensure consistency across models.

Step 4. Produce projections of enrollments by attendance status, sex, age category, and racelethnicity. For each combination of attendance status, sex, age category, and race/ethnicity, enrollment projections were produced by multiplying the projected enrollment rate for that combination by projections of the total population with the corresponding combination of sex, age category, and race/ethnicity.

\section{Data and equations used for the Enrollment in Degree-Granting Institutions by Race/Ethnicity Model}

Enrollment data for degree-granting institutions by race/ethnicity. Enrollment data for 1981 to 2009 by attendance status, sex, and race/ethnicity came from the NCES Integrated Postsecondary Education Data System (IPEDS). These are universe counts. The U.S. Census Bureau, Current Population Survey was the source for enrollment estimates for 1981 to 2009 by the characteristics listed above, as well as age of student.

Population data and projections by race/ethnicity. Population counts for 1981 to 2009 came from the U.S. Census Bureau, Population Estimates series. Population projections for 2010 to 2020 are the Census Bureau's 2008 National Population Projections of the population by sex, age and race/ethnicity (August 2008), ratio-adjusted to line up with most recent historical estimates.

Projections for economic variables. The economic variables used in developing these projections were from the "U.S. Monthly Model: November 2010 Short-Term Projections" from the economic consulting firm, IHS Global Insight.

Estimated equations and model statistics. Tables A-22 through A-29 show the estimated equations and model statistics used to project enrollment rates for the various combinations of attendance status, sex, and race/ethnicity. 


\section{Accuracy of projections for the Degree-Granting Institutions by Race/Ethnicity Model}

Mean absolute percentage errors (MAPEs) for enrollment in degree-granting institutions by race/ethnicity were calculated using the last five editions of Projections of Education Statistics. Table F, below, shows MAPEs for key projections of the Enrollment in Degree-Granting Institutions by Race/Ethnicity Model.

Table F. Mean absolute percentage errors (MAPEs) of projected enrollment in postsecondary degree-granting institutions, by lead time and race/ethnicity: 2011

\begin{tabular}{|c|c|c|c|c|c|c|c|c|c|c|}
\hline \multirow[b]{2}{*}{ Statistic } & \multicolumn{10}{|c|}{ Lead time (years) } \\
\hline & 1 & 2 & 3 & 4 & 5 & 6 & 7 & 8 & 9 & 10 \\
\hline Total enrollment & 1.6 & 2.8 & 3.5 & 4.0 & 5.2 & 6.1 & 8.1 & 9.8 & 11.7 & 11.4 \\
\hline White & 0.4 & 1.1 & 2.3 & 2.8 & 3.6 & - & - & - & - & - \\
\hline Black & 2.2 & 5.3 & 7.5 & 9.2 & 9.4 & - & - & - & - & - \\
\hline Hispanic & 2.0 & 4.7 & 7.0 & 9.0 & 9.5 & - & - & - & - & - \\
\hline Asian/Pacific Islander & 2.2 & 5.2 & 7.1 & 8.3 & 9.2 & - & - & - & - & - \\
\hline American Indian/Alaska Native & 4.4 & 6.4 & 3.9 & 4.3 & 4.6 & - & - & - & - & - \\
\hline Nonresident alien & 2.6 & 6.2 & 8.8 & 6.5 & 2.9 & - & - & - & - & - \\
\hline
\end{tabular}

- Not available.

NOTE: MAPEs for degree-granting institution enrollments were calculated using the last five editions of Projections of Education Statistics. Calculations were made using unrounded numbers. Some data have been revised from previously published figures.

SOURCE: U.S. Department of Education, National Center for Education Statistics, Projections of Education Statistics, various issues. (This table was prepared March 2011.)

\section{The First-Time Freshmen Enrollment in Degree-Granting Institutions Model}

The First-Time Freshmen Enrollment in Degree-Granting Institutions Model produced projections of first-time freshmen enrollment in degree-granting institutions by sex.

\section{Steps used in the First-Time Freshmen Enrollment in Degree-Granting Institutions Model}

The projections were produced in the following manner:

Step 1. Calculate the ratio of first-time freshmen enrollment to undergraduate enrollment. For 1975 to 2009, the ratio of firsttime freshmen enrollment to undergraduate enrollment was calculated for males and females.

Step 2. Project the ratio of first-time freshmen enrollment to undergraduate enrollment. Second, the percentages for both males and females were projected using single exponential smoothing. A separate smoothing constant, chosen to minimize the sum of squared forecast errors, was used for each percentage.

Step 3. Apply the projected ratio to projected undergraduate enrollment. The projected ratios were applied to projections of undergraduate enrollment by sex from the Enrollment in Degree-Granting Institutions Model to yield projections of firsttime freshmen enrollment.

\section{Assumptions underlying this method}

This method assumes that the future pattern in the trend of first-time freshmen enrollment will be the same as that for undergraduate enrollment.

\section{Data used in the First-Time Freshmen Enrollment in Degree-Granting Institutions Model}

Undergraduate and freshmen enrollment data for degree-granting institutions. Undergraduate and freshmen enrollment data by sex for 1975 to 2009 came from the NCES Integrated Postsecondary Education Data System (IPEDS).

Projections of undergraduate enrollment. Projections of undergraduate enrollment by sex came from the Enrollment in Degree-Granting Institutions Model, discussed earlier in this section of appendix A.

\section{Accuracy of projections for the First-Time Freshmen Enrollment Model}

Because this is the third edition of Projections of Education Statistics to include projections of first-time freshmen, there are too few years of data to present the MAPEs. 
Table A-16. Actual and projected numbers for college enrollment rates, by sex, attendance status, and age: Fall 2009, fall 2015, and fall 2020

\begin{tabular}{|c|c|c|c|}
\hline \multirow[b]{2}{*}{ Sex, attendance status, and age } & \multirow[b]{2}{*}{ Actual 2009} & \multicolumn{2}{|c|}{ Projected } \\
\hline & & 2015 & 2020 \\
\hline \multicolumn{4}{|l|}{ Men } \\
\hline \multicolumn{4}{|l|}{ Full-time } \\
\hline 16 years old & 0.5 & 0.5 & 0.5 \\
\hline 17 years old & 2.6 & 2.6 & 2.7 \\
\hline 18 years old & 32.5 & 33.0 & 34.0 \\
\hline 19 years old & 39.4 & 41.8 & 42.9 \\
\hline 20 years old & 37.3 & 37.3 & 38.2 \\
\hline 21 years old & 31.9 & 32.3 & 33.1 \\
\hline 22 years old & 23.5 & 24.5 & 25.2 \\
\hline 23 years old & 16.7 & 17.2 & 17.8 \\
\hline 24 years old & 13.8 & 14.1 & 14.5 \\
\hline 25 to 29 years old & 5.5 & 5.7 & 5.9 \\
\hline 30 to 34 years old & 2.6 & 2.6 & 2.7 \\
\hline 35 to 44 years old & 1.4 & 1.4 & 1.5 \\
\hline \multicolumn{4}{|l|}{ Part-time } \\
\hline 16 years old & 0.3 & 0.3 & 0.3 \\
\hline 17 years old & 0.9 & 0.9 & 0.9 \\
\hline 18 years old & 5.4 & 5.7 & 5.7 \\
\hline 19 years old & 5.6 & 5.9 & 5.9 \\
\hline 20 years old & 9.7 & 9.8 & 9.7 \\
\hline 21 years old & 8.1 & 8.1 & 8.1 \\
\hline 22 years old & 4.8 & 6.0 & 6.0 \\
\hline 23 years old & 7.7 & 7.7 & 7.7 \\
\hline 24 years old & 7.7 & 7.8 & 7.8 \\
\hline 25 to 29 years old & 6.3 & 6.3 & 6.4 \\
\hline 30 to 34 years old & 3.9 & 4.1 & 4.1 \\
\hline 35 to 44 years old & 4.1 & 4.2 & 4.2 \\
\hline \multicolumn{4}{|l|}{ Women } \\
\hline \multicolumn{4}{|l|}{ Full-time } \\
\hline 16 years old & 0.7 & 0.7 & 0.7 \\
\hline 17 years old & 3.5 & 4.0 & 4.4 \\
\hline 18 years old & 44.3 & 49.3 & 51.1 \\
\hline 19 years old & 49.2 & 51.3 & 52.8 \\
\hline 20 years old & 44.4 & 46.5 & 48.0 \\
\hline 21 years old & 40.8 & 42.5 & 44.0 \\
\hline 22 years old & 28.8 & 29.4 & 30.8 \\
\hline 23 years old & 20.0 & 20.9 & 22.0 \\
\hline 24 years old & 15.9 & 16.6 & 17.5 \\
\hline 25 to 29 years old & 7.6 & 8.2 & 8.7 \\
\hline 30 to 34 years old & 3.3 & 3.5 & 3.8 \\
\hline 35 to 44 years old & 3.2 & 3.4 & 3.6 \\
\hline \multicolumn{4}{|l|}{ Part-time } \\
\hline 16 years old & 0.1 & 0.1 & 0.1 \\
\hline 17 years old & 0.4 & 0.5 & 0.6 \\
\hline 18 years old & 7.3 & 7.3 & 7.5 \\
\hline 19 years old & 10.4 & 10.7 & 11.1 \\
\hline 20 years old & 8.4 & 9.9 & 10.3 \\
\hline 21 years old & 9.8 & 10.0 & 10.4 \\
\hline 22 years old & 10.1 & 11.0 & 11.6 \\
\hline 23 years old & 11.2 & 12.2 & 13.0 \\
\hline 24 years old & 10.7 & 11.6 & 12.4 \\
\hline 25 to 29 years old & 7.9 & 8.4 & 9.0 \\
\hline 30 to 34 years old & 6.5 & 6.6 & 7.1 \\
\hline 35 to 44 years old & 8.2 & 9.0 & 9.7 \\
\hline
\end{tabular}

SOURCE: U.S. Department of Education, National Center for Education Statistics, Integrated Postsecondary Education Data System, Spring 2010; Enrollment in Degree-Granting Institutions Model, 1980-2009; and U.S. Department of Commerce, Census Bureau, Current Population Reports,

"Social and Economic Characteristics of Students," 2009. (This table was prepared February 2011.) 
Table A-17. Estimated equations and model statistics for full-time and part-time college enrollment rates of men

\begin{tabular}{|c|c|c|c|c|c|}
\hline Independent variable & Coefficient & Standard error & $t$-statistic & $R^{2}$ & D.W. statistic \\
\hline \multicolumn{6}{|l|}{ Full-time } \\
\hline Intercept term for 17-year-olds & -7.46 & 0.251 & -29.79 & 1.00 & $1.9^{*}$ \\
\hline Intercept term for 18-year-olds & -4.58 & 0.231 & -19.78 & & \\
\hline Intercept term for 19-year-olds & -4.24 & 0.201 & -21.06 & & \\
\hline Intercept term for 20-year-olds & -4.32 & 0.204 & -21.17 & & \\
\hline Intercept term for 21-year-olds & -4.48 & 0.203 & -22.04 & & \\
\hline Intercept term for 22-year-olds & -4.91 & 0.206 & -23.82 & & \\
\hline Intercept term for 23-year-olds & -5.40 & 0.204 & -26.40 & & \\
\hline Intercept term for 24-year-olds & -5.74 & 0.222 & -25.92 & & \\
\hline Intercept term for 25- to 29-year-olds & -6.48 & 0.209 & -31.00 & & \\
\hline Intercept term for 30 - to 34-year-olds & -7.44 & 0.242 & -30.79 & & \\
\hline Intercept term for 35- to 44-year-olds & -7.96 & 0.241 & -32.98 & & \\
\hline \multicolumn{6}{|l|}{$\begin{array}{l}\text { Log of three-period weighted average of per capita } \\
\text { disposable income in } 2000 \text { dollars, using the }\end{array}$} \\
\hline present period and the previous two periods & 0.67 & 0.034 & 20.06 & & \\
\hline Log of age-specific unemployment rate for men & 0.18 & 0.018 & 10.01 & & \\
\hline Autocorrelation coefficient for 17 -year-olds & 0.86 & 0.042 & 20.43 & & \\
\hline Autocorrelation coefficient for 18 -year-olds & 0.88 & 0.047 & 18.56 & & \\
\hline Autocorrelation coefficient for 19 -year-olds & 0.44 & 0.125 & 3.49 & & \\
\hline Autocorrelation coefficient for 20 -year-olds & 0.59 & 0.127 & 4.66 & & \\
\hline Autocorrelation coefficient for 21-year-olds & 0.52 & 0.125 & 4.13 & & \\
\hline Autocorrelation coefficient for 22 -year-olds & 0.73 & 0.109 & 6.70 & & \\
\hline Autocorrelation coefficient for 23-year-olds & 0.65 & 0.120 & 5.42 & & \\
\hline Autocorrelation coefficient for 24-year-olds & 0.83 & 0.113 & 7.34 & & \\
\hline Autocorrelation coefficient for 25- to 29-year-olds & 0.68 & 0.076 & 8.98 & & \\
\hline Autocorrelation coefficient for 30- to 34-year-olds & 0.89 & 0.074 & 11.97 & & \\
\hline Autocorrelation coefficient for 35- to 44-year-olds & 0.87 & 0.068 & 12.81 & & \\
\hline \multicolumn{6}{|l|}{ Part-time } \\
\hline Intercept term for 17 -year-olds & -8.04 & 0.341 & -23.57 & 0.99 & $1.8^{*}$ \\
\hline Intercept term for 18-year-olds & -5.66 & 0.290 & -19.55 & & \\
\hline Intercept term for 19-year-olds & -5.31 & 0.369 & -14.38 & & \\
\hline Intercept term for 20-year-olds & -5.11 & 0.303 & -16.89 & & \\
\hline Intercept term for 21-year-olds & -5.27 & 0.284 & -18.57 & & \\
\hline Intercept term for 22-year-olds & -5.59 & 0.393 & -14.22 & & \\
\hline Intercept term for 23-year-olds & -5.46 & 0.282 & -19.34 & & \\
\hline Intercept term for 24-year-olds & -5.51 & 0.283 & -19.43 & & \\
\hline Intercept term for 25- to 29-year-olds & -5.94 & 0.298 & -19.93 & & \\
\hline Intercept term for 30- to 34-year-olds & -6.39 & 0.299 & -21.40 & & \\
\hline Intercept term for 35- to 44-year-olds & -6.32 & 0.285 & -22.21 & & \\
\hline \multicolumn{6}{|l|}{$\begin{array}{l}\text { Log of three-period weighted average of per capita } \\
\text { disposable income in } 2000 \text { dollars, using the }\end{array}$} \\
\hline present period and the previous two periods & 0.46 & 0.046 & 10.04 & & \\
\hline Log of unemployment rate & 0.16 & 0.021 & 7.44 & & \\
\hline Autocorrelation coefficient for 17 -year-olds & 0.69 & 0.112 & 6.15 & & \\
\hline Autocorrelation coefficient for 18 -year-olds & 0.70 & 0.091 & 7.67 & & \\
\hline Autocorrelation coefficient for 19 -year-olds & 0.92 & 0.057 & 16.02 & & \\
\hline Autocorrelation coefficient for 20 -year-olds & 0.82 & 0.101 & 8.10 & & \\
\hline Autocorrelation coefficient for 21-year-olds & 0.54 & 0.089 & 6.02 & & \\
\hline Autocorrelation coefficient for 22 -year-olds & 0.89 & 0.100 & 8.84 & & \\
\hline Autocorrelation coefficient for 23-year-olds & 0.43 & 0.106 & 4.02 & & \\
\hline Autocorrelation coefficient for 24-year-olds & 0.55 & 0.112 & 4.88 & & \\
\hline Autocorrelation coefficient for 25- to 29-year-olds & 0.89 & 0.044 & 20.32 & & \\
\hline Autocorrelation coefficient for 30- to 34-year-olds & 0.88 & 0.040 & 21.76 & & \\
\hline Autocorrelation coefficient for 35- to 44-year-olds & 0.63 & 0.060 & 10.52 & & \\
\hline
\end{tabular}

${ }^{*} p<.05$.

NOTE: $R^{2}=$ Coefficient of determination. D.W. statistic = Durbin-Watson statistic, a test for autocorrelation among regression residuals. For more details, see Johnston, J., and Dinardo, J. (1996). Econometric Methods, New York: McGraw-Hill. The regression method used to estimate the fulltime and part-time equations was the pooled seemingly unrelated regression method with a first-order autocorrelation correction. The time period used to estimate both equations is from 1981 to 2009, and the number of observations is 319 after the correction for autocorrelation. For additional information, see Intriligator, M.D. (1978). Econometric Models, Techniques, \& Applications. New Jersey: Prentice-Hall, Inc., pp. 165-173.

SOURCE: U.S. Department of Education, National Center for Education Statistics, Enrollment in Degree-Granting Institutions Model, $1980-2009$.

(This table was prepared January 2011.) 
Table A-18. Estimated equations and model statistics for full-time and part-time college enrollment rates of women

\begin{tabular}{|c|c|c|c|c|c|}
\hline Independent variable & Coefficient & Standard error & $t$-statistic & $R^{2}$ & D.W. statistic \\
\hline \multicolumn{6}{|l|}{ Full-time } \\
\hline Intercept term for 17-year-olds & -9.48 & 0.181 & -52.48 & 1.00 & $1.29^{\star \star}$ \\
\hline Intercept term for 18-year-olds & -6.70 & 0.163 & -41.05 & & \\
\hline Intercept term for 19-year-olds & -6.58 & 0.159 & -41.37 & & \\
\hline Intercept term for 20-year-olds & -6.70 & 0.159 & -42.22 & & \\
\hline Intercept term for 21-year-olds & -6.92 & 0.159 & -43.57 & & \\
\hline Intercept term for 22-year-olds & -7.58 & 0.160 & -47.26 & & \\
\hline Intercept term for 23-year-olds & -8.08 & 0.162 & -49.98 & & \\
\hline Intercept term for 24-year-olds & -8.46 & 0.163 & -51.90 & & \\
\hline Intercept term for 25- to 29-year-olds & -9.05 & 0.164 & -55.20 & & \\
\hline Intercept term for 30- to 34-year-olds & -9.76 & 0.162 & -60.11 & & \\
\hline Intercept term for 35- to 44-year-olds & -9.98 & 0.163 & -61.28 & & \\
\hline \multicolumn{6}{|l|}{$\begin{array}{l}\text { Log of three-period weighted average of per capita } \\
\text { disposable income in } 2000 \text { dollars, using the }\end{array}$} \\
\hline present period and the previous two periods & 1.17 & 0.031 & 37.27 & & \\
\hline Log of age-specific unemployment rate for women & 0.28 & 0.041 & 6.79 & & \\
\hline \multicolumn{6}{|l|}{ Part-time } \\
\hline Intercept term for 17-year-olds & -11.18 & 0.500 & -22.38 & 0.99 & $1.88^{*}$ \\
\hline Intercept term for 18-year-olds & -8.46 & 0.297 & -28.54 & & \\
\hline Intercept term for 19 -year-olds & -7.95 & 0.287 & -27.73 & & \\
\hline Intercept term for 20-year-olds & -8.12 & 0.284 & -28.56 & & \\
\hline Intercept term for 21-year-olds & -8.12 & 0.280 & -29.06 & & \\
\hline Intercept term for 22-year-olds & -8.29 & 0.280 & -29.58 & & \\
\hline Intercept term for 23-year-olds & -8.36 & 0.282 & -29.63 & & \\
\hline Intercept term for 24-year-olds & -8.42 & 0.286 & -29.46 & & \\
\hline Intercept term for 25- to 29-year-olds & -8.88 & 0.295 & -30.08 & & \\
\hline Intercept term for 30- to 34-year-olds & -9.25 & 0.292 & -31.70 & & \\
\hline Intercept term for 35- to 44-year-olds & -8.95 & 0.296 & -30.23 & & \\
\hline \multicolumn{6}{|l|}{$\begin{array}{l}\text { Log of three-period weighted average of per capita } \\
\text { disposable income in } 2000 \text { dollars, using the }\end{array}$} \\
\hline present period and the previous two periods & 1.01 & 0.044 & 22.70 & & \\
\hline Log of unemployment rate & 0.14 & 0.024 & 5.85 & & \\
\hline Autocorrelation coefficient for 17 -year-olds & 0.82 & 0.074 & 11.11 & & \\
\hline Autocorrelation coefficient for 18 -year-olds & 0.75 & 0.082 & 9.11 & & \\
\hline Autocorrelation coefficient for 19-year-olds & 0.77 & 0.071 & 10.71 & & \\
\hline Autocorrelation coefficient for 20 -year-olds & 0.60 & 0.105 & 5.74 & & \\
\hline Autocorrelation coefficient for 21 -year-olds & 0.30 & 0.123 & 2.39 & & \\
\hline Autocorrelation coefficient for 22-year-olds & 0.44 & 0.097 & 4.57 & & \\
\hline Autocorrelation coefficient for 23-year-olds & 0.52 & 0.077 & 6.82 & & \\
\hline Autocorrelation coefficient for 24-year-olds & 0.74 & 0.063 & 11.74 & & \\
\hline Autocorrelation coefficient for 25- to 29-year-olds & 0.89 & 0.034 & 25.99 & & \\
\hline Autocorrelation coefficient for 30 - to 34 -year-olds & 0.89 & 0.026 & 33.81 & & \\
\hline Autocorrelation coefficient for 35- to 44-year-olds & 0.89 & 0.026 & 34.19 & & \\
\hline
\end{tabular}

\footnotetext{
${ }^{*} p<.05$.

** Inconclusive.

NOTE: $R^{2}=$ Coefficient of determination. D.W. statistic = Durbin-Watson statistic, a test for autocorrelation among regression residuals. For more details, see Johnston, J., and Dinardo, J. (1996). Econometric Methods, New York: McGraw-Hill. The regression method used to estimate the full-time equation was the pooled seemingly unrelated regression method. The regression method used to estimate the part-time equation was the pooled seemingly unrelated regression method with a first-order autocorrelation correction. The time period used to estimate the full-time equation was from 1980 to 2009 and that for the part-time equation was from 1981 to 2009 . The number of observations for the full-time equation is 330 and the number of observations for the part-time equation, after the correction for autocorrelation, is 319. For additional information, see Intriligator, M.D. (1978). Econometric Models, Techniques, \& Applications. New Jersey: Prentice-Hall, Inc., pp. 165-173.

SOURCE: U.S. Department of Education, National Center for Education Statistics, Enrollment in Degree-Granting Institutions Model, $1980-2008$. (This table was prepared March 2011.)
} 
Table A-19. Actual and projected percentages of full-time students at postsecondary degree-granting institutions, by sex, age group, student level, and institution level: Fall 2009, and fall 2010 through fall 2020

\begin{tabular}{|c|c|c|c|c|}
\hline \multirow[b]{2}{*}{ Age and institution type } & \multicolumn{2}{|c|}{ Men } & \multicolumn{2}{|c|}{ Women } \\
\hline & Actual 2009 & $\begin{array}{r}\text { Projected } 2010 \\
\text { through } 2020 \\
\end{array}$ & Actual 2009 & $\begin{array}{r}\text { Projected } 2010 \\
\text { through } 2020 \\
\end{array}$ \\
\hline \multicolumn{5}{|l|}{18 and 19 years old } \\
\hline Undergraduate, 4-year institutions & 66.3 & 65.9 & 65.0 & 65.4 \\
\hline Undergraduate, 2-year institutions & 33.9 & 33.8 & 34.2 & 34.0 \\
\hline Postbaccalaureate, 4-year institutions & \# & 0.3 & 0.9 & 0.5 \\
\hline \multicolumn{5}{|l|}{20 and 21 years old } \\
\hline Undergraduate, 4-year institutions & 76.8 & 76.1 & 80.0 & 80.1 \\
\hline Undergraduate, 2-year institutions & 23.0 & 22.6 & 17.9 & 17.9 \\
\hline Postbaccalaureate, 4-year institutions & 0.2 & 1.3 & 2.1 & 1.9 \\
\hline \multicolumn{5}{|l|}{22 to 24 years old } \\
\hline Undergraduate, 4-year institutions & 60.6 & 60.7 & 61.3 & 60.9 \\
\hline Undergraduate, 2-year institutions & 19.1 & 19.1 & 17.1 & 17.0 \\
\hline Postbaccalaureate, 4-year institutions & 20.3 & 20.2 & 21.6 & 22.1 \\
\hline \multicolumn{5}{|l|}{25 to 29 years old } \\
\hline Undergraduate, 4-year institutions & 40.4 & 40.8 & 39.6 & 39.6 \\
\hline Undergraduate, 2-year institutions & 22.4 & 21.1 & 24.7 & 24.7 \\
\hline Postbaccalaureate, 4-year institutions & 37.3 & 38.1 & 35.7 & 35.7 \\
\hline \multicolumn{5}{|l|}{30 to 34 years old } \\
\hline Undergraduate, 4-year institutions & 38.2 & 38.1 & 39.0 & 39.0 \\
\hline Undergraduate, 2-year institutions & 25.6 & 25.7 & 32.5 & 32.5 \\
\hline Postbaccalaureate, 4-year institutions & 36.2 & 36.2 & 28.5 & 28.5 \\
\hline \multicolumn{5}{|l|}{35 years and over } \\
\hline Undergraduate, 4-year institutions & 40.2 & 40.2 & 45.9 & 45.6 \\
\hline Undergraduate, 2-year institutions & 22.9 & 22.9 & 31.9 & 31.7 \\
\hline Postbaccalaureate, 4-year institutions & 36.9 & 36.8 & 22.2 & 22.6 \\
\hline
\end{tabular}

\# Rounds to zero.

NOTE: Detail may not sum to totals because of rounding.

SOURCE: U.S. Department of Education, National Center for Education Statistics, Integrated Postsecondary Education Data System, Spring 2010;

Enrollment in Degree-Granting Institutions Model, 1980-2009; and U.S. Department of Commerce, Census Bureau, Current Population Reports,

"Social and Economic Characteristics of Students," 2009. (This table was prepared February 2011.) 
Table A-20. Actual and projected percentages of part-time students at postsecondary degree-granting institutions, by sex, age group, level enrolled, and level of institution: Fall 2009, and fall 2010 through fall 2020

\begin{tabular}{|c|c|c|c|c|}
\hline \multirow[b]{2}{*}{ Age group and level of institution } & \multicolumn{2}{|c|}{ Men } & \multicolumn{2}{|c|}{ Women } \\
\hline & Actual 2009 & $\begin{array}{r}\text { Projected } 2010 \\
\text { through } 2020 \\
\end{array}$ & Actual 2009 & $\begin{array}{r}\text { Projected } 2010 \\
\text { through } 2020 \\
\end{array}$ \\
\hline \multicolumn{5}{|l|}{18 and 19 years old } \\
\hline Undergraduate, 4-year institutions & 11.8 & 11.8 & 18.5 & 18.5 \\
\hline Undergraduate, 2-year institutions & 87.7 & 87.8 & 81.4 & 81.1 \\
\hline Postbaccalaureate, 4-year institutions & 0.5 & 0.5 & 0.1 & 0.4 \\
\hline \multicolumn{5}{|l|}{20 and 21 years old } \\
\hline Undergraduate, 4-year institutions & 26.6 & 26.6 & 28.3 & 29.9 \\
\hline Undergraduate, 2-year institutions & 72.7 & 72.6 & 70.8 & 69.1 \\
\hline Postbaccalaureate, 4-year institutions & 0.7 & 0.8 & 0.9 & 1.1 \\
\hline \multicolumn{5}{|l|}{22 to 24 years old } \\
\hline Undergraduate, 4-year institutions & 32.8 & 33.0 & 35.8 & 35.7 \\
\hline Undergraduate, 2-year institutions & 55.5 & 56.3 & 52.6 & 52.2 \\
\hline Postbaccalaureate, 4-year institutions & 11.7 & 10.8 & 11.6 & 12.1 \\
\hline \multicolumn{5}{|l|}{25 to 29 years old } \\
\hline Undergraduate, 4-year institutions & 29.4 & 29.4 & 26.3 & 26.3 \\
\hline Undergraduate, 2-year institutions & 54.0 & 54.0 & 54.7 & 54.7 \\
\hline Postbaccalaureate, 4-year institutions & 16.6 & 16.6 & 18.9 & 19.0 \\
\hline \multicolumn{5}{|l|}{30 to 34 years old } \\
\hline Undergraduate, 4-year institutions & 34.0 & 33.2 & 27.9 & 27.9 \\
\hline Undergraduate, 2-year institutions & 44.5 & 45.6 & 50.0 & 50.0 \\
\hline Postbaccalaureate, 4-year institutions & 21.6 & 21.2 & 22.2 & 22.2 \\
\hline \multicolumn{5}{|l|}{35 years and over } \\
\hline Undergraduate, 4-year institutions & 27.2 & 27.1 & 27.4 & 27.4 \\
\hline Undergraduate, 2-year institutions & 47.4 & 47.1 & 47.6 & 47.8 \\
\hline Postbaccalaureate, 4-year institutions & 25.4 & 25.8 & 25.0 & 24.8 \\
\hline
\end{tabular}

NOTE: Detail may not sum to totals because of rounding.

SOURCE: U.S. Department of Education, National Center for Education Statistics, Integrated Postsecondary Education Data System, Spring 2010; Enrollment in Degree-Granting Institutions Model, 1980-2009; and U.S. Department of Commerce, Census Bureau, Current Population Reports,

"Social and Economic Characteristics of Students," 2009. (This table was prepared February 2011.)

Table A-21. Actual and projected enrollment in public postsecondary degree-granting institutions as a percent of total postsecondary enrollment, by sex, attendance status, level enrolled, and level of institution: Fall 2009, and fall 2010 through fall 2020

\begin{tabular}{lrrr}
\hline & \multicolumn{2}{c}{ Men } & Women \\
\cline { 2 - 3 } Enrollment category & $\begin{array}{r}\text { Projected 2010 } \\
\text { through 2020 }\end{array}$ & $\begin{array}{r}\text { Projected 2010 } \\
\text { through 2020 }\end{array}$ \\
\hline Full-time, undergraduate, 4-year institutions & Actual 2009 & 64.8 & 60.0 \\
Part-time, undergraduate, 4-year institutions & 64.8 & 66.8 & 62.5 \\
Full-time, undergraduate, 2-year institutions & 66.8 & 91.0 & 86.8 \\
Part-time, undergraduate, 2-year institutions & 91.0 & 99.1 & 98.6 \\
Full-time, postbaccalaureate, 4-year institutions & 99.1 & 49.1 & 45.7 \\
Part-time, postbaccalaureate, 4-year institutions & 49.1 & 52.5 & 98.6 \\
\hline
\end{tabular}

SOURCE: U.S. Department of Education, National Center for Education Statistics, Integrated Postsecondary Education Data System, Spring 2010; and Enrollment in Degree-Granting Institutions Model, 1980-2009. (This table was prepared February 2011.) 
Table A-22. Estimated equations and model statistics for full-time and part-time college enrollment rates of White men

\begin{tabular}{|c|c|c|c|c|c|}
\hline Independent variable & Coefficient & Standard error & $t$-statistic & $R^{2}$ & D.W. statistic \\
\hline \multicolumn{6}{|l|}{ Full-time } \\
\hline Intercept term for 17-year-olds & -8.69 & 0.179 & -48.41 & 1.00 & $1.46^{\star \star}$ \\
\hline Intercept term for 18-year-olds & -5.65 & 0.159 & -35.48 & & \\
\hline Intercept term for 19-year-olds & -5.43 & 0.155 & -34.92 & & \\
\hline Intercept term for 20-year-olds & -5.62 & 0.156 & -36.02 & & \\
\hline Intercept term for 21-year-olds & -5.76 & 0.156 & -37.01 & & \\
\hline Intercept term for 22-year-olds & -6.25 & 0.156 & -39.97 & & \\
\hline Intercept term for 23-year-olds & -6.80 & 0.156 & -43.45 & & \\
\hline Intercept term for 24-year-olds & -7.23 & 0.158 & -45.72 & & \\
\hline Intercept term for 25- to 29 -year-olds & -8.08 & 0.156 & -51.73 & & \\
\hline Intercept term for 30- to 34-year-olds & -9.11 & 0.159 & -57.28 & & \\
\hline Intercept term for 35- to 44-year-olds & -9.74 & 0.160 & -60.83 & & \\
\hline \multicolumn{6}{|l|}{ Log of White per capita disposable } \\
\hline income in current dollars & 0.26 & 0.008 & 32.35 & & \\
\hline \multicolumn{6}{|l|}{ Part-time } \\
\hline Intercept term for 17-year-olds & -5.21 & 0.149 & -35.04 & 0.99 & $1.47^{\star}$ \\
\hline Intercept term for 18-year-olds & -1.87 & 0.076 & -24.70 & & \\
\hline Intercept term for 19-year-olds & -1.49 & 0.091 & -16.30 & & \\
\hline Intercept term for 20-year-olds & -1.48 & 0.076 & -19.44 & & \\
\hline Intercept term for 21-year-olds & -1.52 & 0.077 & -19.72 & & \\
\hline Intercept term for 22-year-olds & -1.71 & 0.076 & -22.45 & & \\
\hline Intercept term for 23-year-olds & -1.72 & 0.071 & -24.32 & & \\
\hline Intercept term for 24-year-olds & -1.74 & 0.071 & -24.62 & & \\
\hline Intercept term for 25- to 29-year-olds & -2.07 & 0.070 & -29.50 & & \\
\hline Intercept term for 30- to 34-year-olds & -2.53 & 0.073 & -34.65 & & \\
\hline Intercept term for 35- to 44-year-olds & -2.56 & 0.069 & -37.36 & & \\
\hline employment cost index & 0.95 & 0.088 & 10.74 & & \\
\hline
\end{tabular}

${ }^{*} p<.05$.

** Inconclusive.

NOTE: $R^{2}=$ Coefficient of determination. D.W. statistic = Durbin-Watson statistic, a test for autocorrelation among regression residuals. For more details, see Johnston, J., and Dinardo, J. (1996). Econometric Methods, New York: McGraw-Hill. The regression method used to estimate the full-time and part-time equations was the pooled seemingly unrelated regression method. The time period used to estimate the equations is from 1980 to 2009. The number of observations is 330 . For additional information, see Intriligator, M.D. (1978). Econometric Models, Techniques, \& Applications. New Jersey: Prentice-Hall, Inc., pp. 165-173. Race categories exclude persons of Hispanic ethnicity.

SOURCE: U.S. Department of Education, National Center for Education Statistics, Enrollment in Degree-Granting Institutions by Race/Ethnicity Model, 1980-2009. (This table was prepared March 2011.) 
Table A-23. Estimated equations and model statistics for full-time and part-time college enrollment rates of White women

\begin{tabular}{|c|c|c|c|c|c|}
\hline Independent variable & Coefficient & Standard error & $t$-statistic & $R^{2}$ & D.W. statistic \\
\hline \multicolumn{6}{|l|}{ Full-time } \\
\hline Intercept term for 17-year-olds & -13.57 & 0.264 & -51.31 & 1.00 & $1.50^{*}$ \\
\hline Intercept term for 18-year-olds & -10.61 & 0.248 & -42.84 & & \\
\hline Intercept term for 19-year-olds & -10.49 & 0.245 & -42.85 & & \\
\hline Intercept term for 20-year-olds & -10.74 & 0.245 & -43.80 & & \\
\hline Intercept term for 21-year-olds & -10.99 & 0.246 & -44.74 & & \\
\hline Intercept term for 22-year-olds & -11.72 & 0.246 & -47.58 & & \\
\hline Intercept term for 23-year-olds & -12.29 & 0.248 & -49.62 & & \\
\hline Intercept term for 24-year-olds & -12.69 & 0.248 & -51.22 & & \\
\hline Intercept term for 25- to 29-year-olds & -13.50 & 0.246 & -54.83 & & \\
\hline Intercept term for 30- to 34-year-olds & -14.22 & 0.245 & -57.95 & & \\
\hline Intercept term for 35- to 44-year-olds & -14.39 & 0.246 & -58.52 & & \\
\hline \multicolumn{6}{|l|}{ Log of White per capita disposable } \\
\hline income in current dollars & 0.54 & 0.013 & 42.42 & & \\
\hline \multicolumn{6}{|l|}{ Part-time } \\
\hline Intercept term for 17-year-olds & -9.04 & 0.311 & -29.12 & 0.79 & $1.55^{\star}$ \\
\hline Intercept term for 18-year-olds & -5.77 & 0.253 & -22.84 & & \\
\hline Intercept term for 19-year-olds & -5.32 & 0.256 & -20.77 & & \\
\hline Intercept term for 20-year-olds & -5.41 & 0.255 & -21.22 & & \\
\hline Intercept term for 21-year-olds & -5.48 & 0.253 & -21.62 & & \\
\hline Intercept term for 22-year-olds & -5.67 & 0.252 & -22.46 & & \\
\hline Intercept term for 23-year-olds & -5.72 & 0.252 & -22.66 & & \\
\hline Intercept term for 24-year-olds & -5.74 & 0.252 & -22.79 & & \\
\hline Intercept term for 25- to 29-year-olds & -6.07 & 0.251 & -24.21 & & \\
\hline Intercept term for 30- to 34-year-olds & -6.39 & 0.252 & -25.36 & & \\
\hline Intercept term for 35- to 44-year-olds & -6.08 & 0.251 & -24.24 & & \\
\hline \multicolumn{6}{|l|}{ Log of real total private compensation } \\
\hline employment cost index & 0.18 & 0.013 & 14.00 & & \\
\hline
\end{tabular}

${ }^{*} p<.05$.

NOTE: $R^{2}$ = Coefficient of determination. D.W. statistic = Durbin-Watson statistic, a test for autocorrelation among regression residuals. For more details, see Johnston, J., and Dinardo, J. (1996). Econometric Methods, New York: McGraw-Hill. The regression method used to estimate the full-time and part-time equations was the pooled seemingly unrelated regression method. The time period used to estimate the equations is from 1980 to 2009. The number of observations is 330. For additional information, see Intriligator, M.D. (1978). Econometric Models, Techniques, \& Applications. New Jersey: Prentice-Hall, Inc., pp. 165-173. Race categories exclude persons of Hispanic ethnicity.

SOURCE: U.S. Department of Education, National Center for Education Statistics, Enrollment in Degree-Granting Institutions by Race/Ethnicity Model, 1980-2009. (This table was prepared March 2011.) 
Table A-24. Estimated equations and model statistics for full-time and part-time college enrollment rates of Black men

\begin{tabular}{|c|c|c|c|c|c|}
\hline Independent variable & Coefficient & Standard error & $t$-statistic & $R^{2}$ & D.W. statistic \\
\hline \multicolumn{6}{|l|}{ Full-time } \\
\hline Intercept term for 17 -year-olds & -9.90 & 0.521 & -19.01 & 0.97 & $1.84^{*}$ \\
\hline Intercept term for 18-year-olds & -7.70 & 0.516 & -14.92 & & \\
\hline Intercept term for 19-year-olds & -7.43 & 0.516 & -14.40 & & \\
\hline Intercept term for 20-year-olds & -7.51 & 0.517 & -14.54 & & \\
\hline Intercept term for 21-year-olds & -7.81 & 0.517 & -15.11 & & \\
\hline Intercept term for 22-year-olds & -7.96 & 0.518 & -15.37 & & \\
\hline Intercept term for 23-year-olds & -8.48 & 0.520 & -16.29 & & \\
\hline Intercept term for 24-year-olds & -8.74 & 0.518 & -16.87 & & \\
\hline Intercept term for 25- to 29-year-olds & -9.52 & 0.518 & -18.38 & & \\
\hline Intercept term for 30- to 34-year-olds & -10.30 & 0.523 & -19.69 & & \\
\hline Intercept term for 35- to 44-year-olds & -10.69 & 0.519 & -20.58 & & \\
\hline \multicolumn{6}{|l|}{ Log of Black per capita disposable } \\
\hline income in current dollars & 0.33 & 0.028 & 11.83 & & \\
\hline \multicolumn{6}{|l|}{ Part-time } \\
\hline Intercept term for 17 -year-olds & -10.02 & 0.354 & -28.31 & 0.65 & $1.84^{*}$ \\
\hline Intercept term for 18 -year-olds & -9.17 & 0.381 & -24.07 & & \\
\hline Intercept term for 19-year-olds & -8.36 & 0.360 & -23.19 & & \\
\hline Intercept term for 20-year-olds & -8.27 & 0.359 & -23.02 & & \\
\hline Intercept term for 21-year-olds & -8.26 & 0.349 & -23.70 & & \\
\hline Intercept term for 22-year-olds & -8.32 & 0.363 & -22.92 & & \\
\hline Intercept term for 23-year-olds & -8.53 & 0.370 & -23.03 & & \\
\hline Intercept term for 24-year-olds & -8.64 & 0.379 & -22.76 & & \\
\hline Intercept term for 25- to 29 -year-olds & -8.64 & 0.348 & -24.82 & & \\
\hline Intercept term for 30- to 34-year-olds & -8.85 & 0.347 & -25.53 & & \\
\hline Intercept term for 35- to 44-year-olds & -8.92 & 0.344 & -25.92 & & \\
\hline \multicolumn{6}{|l|}{ Log of Black per capita disposable } \\
\hline income in current dollars & 0.29 & 0.018 & 15.64 & & \\
\hline
\end{tabular}

${ }^{*} p<.05$.

NOTE: $R^{2}$ = Coefficient of determination. D.W. statistic = Durbin-Watson statistic, a test for autocorrelation among regression residuals. For more details, see Johnston, J., and Dinardo, J. (1996). Econometric Methods, New York: McGraw-Hill. The regression method used to estimate the full-time and part-time equations was the pooled seemingly unrelated regression method. The time period used to estimate the equations is from 1980 to 2009. The number of observations is 330. For additional information, see Intriligator, M.D. (1978). Econometric Models, Techniques, \& Applications. New Jersey: Prentice-Hall, Inc., pp. 165-173. Race categories exclude persons of Hispanic ethnicity.

SOURCE: U.S. Department of Education, National Center for Education Statistics, Enrollment in Degree-Granting Institutions by Race/Ethnicity Model, 1980-2009. (This table was prepared March 2011.) 
Table A-25. Estimated equations and model statistics for full-time and part-time college enrollment rates of Black women

\begin{tabular}{|c|c|c|c|c|c|}
\hline Independent variable & Coefficient & Standard error & $t$-statistic & $R^{2}$ & D.W. statistic \\
\hline \multicolumn{6}{|l|}{ Full-time } \\
\hline Intercept term for 17-year-olds & -15.03 & 0.621 & -24.19 & 0.97 & $1.74^{*}$ \\
\hline Intercept term for 18-year-olds & -12.85 & 0.615 & -20.89 & & \\
\hline Intercept term for 19-year-olds & -12.63 & 0.615 & -20.55 & & \\
\hline Intercept term for 20-year-olds & -12.90 & 0.615 & -20.99 & & \\
\hline Intercept term for 21-year-olds & -13.05 & 0.614 & -21.24 & & \\
\hline Intercept term for 22-year-olds & -13.50 & 0.615 & -21.97 & & \\
\hline Intercept term for 23-year-olds & -13.81 & 0.615 & -22.46 & & \\
\hline Intercept term for 24-year-olds & -14.17 & 0.616 & -23.00 & & \\
\hline Intercept term for 25- to 29-year-olds & -14.93 & 0.617 & -24.22 & & \\
\hline Intercept term for 30- to 34-year-olds & -15.41 & 0.615 & -25.05 & & \\
\hline Intercept term for 35- to 44-year-olds & -15.77 & 0.616 & -25.62 & & \\
\hline \multicolumn{6}{|l|}{ Log of Black per capita disposable } \\
\hline income in current dollars & 0.64 & 0.033 & 19.35 & & \\
\hline \multicolumn{6}{|l|}{ Part-time } \\
\hline Intercept term for 17 -year-olds & -14.56 & 0.665 & -21.91 & 0.62 & $1.74^{*}$ \\
\hline Intercept term for 18-year-olds & -13.12 & 0.664 & -19.78 & & \\
\hline Intercept term for 19-year-olds & -12.71 & 0.664 & -19.16 & & \\
\hline Intercept term for 20-year-olds & -12.78 & 0.662 & -19.31 & & \\
\hline Intercept term for 21-year-olds & -12.66 & 0.663 & -19.11 & & \\
\hline Intercept term for 22-year-olds & -12.66 & 0.663 & -19.10 & & \\
\hline Intercept term for 23-year-olds & -12.67 & 0.662 & -19.15 & & \\
\hline Intercept term for 24-year-olds & -12.85 & 0.662 & -19.41 & & \\
\hline Intercept term for 25- to 29-year-olds & -13.02 & 0.658 & -19.79 & & \\
\hline Intercept term for 30- to 34-year-olds & -13.13 & 0.659 & -19.93 & & \\
\hline Intercept term for 35- to 44-year-olds & -13.01 & 0.658 & -19.78 & & \\
\hline \multicolumn{6}{|l|}{ Log of Black per capita disposable } \\
\hline income in current dollars & 0.55 & 0.035 & 15.58 & & \\
\hline
\end{tabular}

${ }^{*} p<.05$.

NOTE: $R^{2}=$ Coefficient of determination. D.W. statistic $=$ Durbin-Watson statistic, a test for autocorrelation among regression residuals. For more details, see Johnston, J., and Dinardo, J. (1996). Econometric Methods, New York: McGraw-Hill. The regression method used to estimate the full-time and part-time equations was the pooled seemingly unrelated regression method. The time period used to estimate the equations is from 1980 to 2009. The number of observations is 330. For additional information, see Intriligator, M.D. (1978). Econometric Models, Techniques, \& Applications. New Jersey: Prentice-Hall, Inc., pp. 165-173. Race categories exclude persons of Hispanic ethnicity.

SOURCE: U.S. Department of Education, National Center for Education Statistics, Enrollment in Degree-Granting Institutions by Race/Ethnicity Model, 1980-2009. (This table was prepared March 2011.) 
Table A-26. Estimated equations and model statistics for full-time and part-time college enrollment rates of Hispanic men

\begin{tabular}{|c|c|c|c|c|c|}
\hline Independent variable & Coefficient & Standard error & $t$-statistic & $R^{2}$ & D.W. statistic \\
\hline \multicolumn{6}{|l|}{ Full-time } \\
\hline Intercept term for 17-year-olds & -11.06 & 0.604 & -18.32 & 0.94 & $1.89^{\star}$ \\
\hline Intercept term for 18-year-olds & -9.09 & 0.596 & -15.24 & & \\
\hline Intercept term for 19-year-olds & -8.87 & 0.596 & -14.88 & & \\
\hline Intercept term for 20-year-olds & -9.08 & 0.596 & -15.25 & & \\
\hline Intercept term for 21-year-olds & -9.30 & 0.599 & -15.54 & & \\
\hline Intercept term for 22-year-olds & -9.77 & 0.597 & -16.36 & & \\
\hline Intercept term for 23-year-olds & -10.06 & 0.598 & -16.83 & & \\
\hline Intercept term for 24-year-olds & -10.20 & 0.597 & -17.08 & & \\
\hline Intercept term for 25- to 29-year-olds & -11.04 & 0.598 & -18.46 & & \\
\hline Intercept term for 30- to 34-year-olds & -11.85 & 0.599 & -19.78 & & \\
\hline Intercept term for 35- to 44-year-olds & -12.34 & 0.603 & -20.45 & & \\
\hline \multicolumn{6}{|l|}{ Log of Hispanic per capita disposable } \\
\hline income in current dollars & 0.39 & 0.033 & 12.02 & & \\
\hline \multicolumn{6}{|l|}{ Part-time } \\
\hline Intercept term for 17-year-olds & -10.55 & 0.404 & -26.09 & 0.73 & $1.79^{*}$ \\
\hline Intercept term for 18-year-olds & -8.98 & 0.401 & -22.39 & & \\
\hline Intercept term for 19-year-olds & -8.66 & 0.408 & -21.21 & & \\
\hline Intercept term for 20 -year-olds & -8.53 & 0.401 & -21.26 & & \\
\hline Intercept term for 21-year-olds & -8.57 & 0.400 & -21.41 & & \\
\hline Intercept term for 22-year-olds & -8.97 & 0.399 & -22.47 & & \\
\hline Intercept term for 23-year-olds & -8.93 & 0.408 & -21.90 & & \\
\hline Intercept term for 24-year-olds & -8.95 & 0.399 & -22.45 & & \\
\hline Intercept term for 25- to 29-year-olds & -9.32 & 0.390 & -23.92 & & \\
\hline Intercept term for 30- to 34-year-olds & -9.78 & 0.392 & -24.93 & & \\
\hline Intercept term for 35- to 44-year-olds & -9.80 & 0.390 & -25.16 & & \\
\hline \multicolumn{6}{|l|}{ Log of Hispanic per capita disposable } \\
\hline income in current dollars & 0.32 & 0.021 & 15.27 & & \\
\hline
\end{tabular}

${ }^{*} p<.05$.

NOTE: $R^{2}=$ Coefficient of determination. D.W. statistic = Durbin-Watson statistic, a test for autocorrelation among regression residuals. For more details, see Johnston, J., and Dinardo, J. (1996). Econometric Methods, New York: McGraw-Hill. The regression method used to estimate the full-time and part-time equations was the pooled seemingly unrelated regression method. The time period used to estimate the equations is from 1980 to 2009. The number of observations is 330. For additional information, see Intriligator, M.D. (1978). Econometric Models, Techniques, \& Applications. New Jersey: Prentice-Hall, Inc., pp. 165-173.

SOURCE: U.S. Department of Education, National Center for Education Statistics, Enrollment in Degree-Granting Institutions by Race/Ethnicity Model, 1980-2009. (This table was prepared March 2011.) 
Table A-27. Estimated equations and model statistics for full-time and part-time college enrollment rates of Hispanic women

\begin{tabular}{|c|c|c|c|c|c|}
\hline Independent variable & Coefficient & Standard error & $t$-statistic & $R^{2}$ & D.W. statistic \\
\hline \multicolumn{6}{|l|}{ Full-time } \\
\hline Intercept term for 17-year-olds & -17.63 & 0.466 & -37.82 & 0.95 & $1.88^{*}$ \\
\hline Intercept term for 18-year-olds & -15.11 & 0.446 & -33.86 & & \\
\hline Intercept term for 19-year-olds & -15.03 & 0.444 & -33.84 & & \\
\hline Intercept term for 20-year-olds & -15.36 & 0.445 & -34.51 & & \\
\hline Intercept term for 21-year-olds & -15.50 & 0.445 & -34.80 & & \\
\hline Intercept term for 22-year-olds & -16.06 & 0.449 & -35.76 & & \\
\hline Intercept term for 23-year-olds & -16.38 & 0.446 & -36.69 & & \\
\hline Intercept term for 24-year-olds & -16.83 & 0.453 & -37.12 & & \\
\hline Intercept term for 25- to 29-year-olds & -17.46 & 0.444 & -39.29 & & \\
\hline Intercept term for 30- to 34-year-olds & -18.18 & 0.448 & -40.59 & & \\
\hline Intercept term for 35- to 44-year-olds & -18.44 & 0.450 & -40.96 & & \\
\hline \multicolumn{6}{|l|}{ Log of Hispanic per capita disposable } \\
\hline income in current dollars & 0.75 & 0.024 & 31.18 & & \\
\hline \multicolumn{6}{|l|}{ Part-time } \\
\hline Intercept term for 17-year-olds & -15.17 & 0.466 & -32.55 & 0.75 & $1.84^{\star}$ \\
\hline Intercept term for 18-year-olds & -13.04 & 0.453 & -28.77 & & \\
\hline Intercept term for 19-year-olds & -12.73 & 0.448 & -28.41 & & \\
\hline Intercept term for 20-year-olds & -12.99 & 0.456 & -28.52 & & \\
\hline Intercept term for 21-year-olds & -12.88 & 0.457 & -28.17 & & \\
\hline Intercept term for 22-year-olds & -13.16 & 0.456 & -28.84 & & \\
\hline Intercept term for 23-year-olds & -12.98 & 0.450 & -28.85 & & \\
\hline Intercept term for 24-year-olds & -13.26 & 0.454 & -29.18 & & \\
\hline Intercept term for 25- to 29 -year-olds & -13.57 & 0.444 & -30.57 & & \\
\hline Intercept term for 30- to 34-year-olds & -13.94 & 0.444 & -31.42 & & \\
\hline Intercept term for 35- to 44-year-olds & -13.77 & 0.443 & -31.11 & & \\
\hline \multicolumn{6}{|l|}{ Log of Hispanic per capita disposable } \\
\hline income in current dollars & 0.58 & 0.024 & 23.85 & & \\
\hline
\end{tabular}

${ }^{*} p<.05$

NOTE: $R^{2}=$ Coefficient of determination. D.W. statistic $=$ Durbin-Watson statistic, a test for autocorrelation among regression residuals. For more details, see Johnston, J., and Dinardo, J. (1996). Econometric Methods, New York: McGraw-Hill. The regression method used to estimate the full-time and part-time equations was the pooled seemingly unrelated regression method. The time period used to estimate the equations is from 1980 to 2009. The number of observations is 330. For additional information, see Intriligator, M.D. (1978). Econometric Models, Techniques, \& Applications. New Jersey: Prentice-Hall, Inc., pp. 165-173.

SOURCE: U.S. Department of Education, National Center for Education Statistics, Enrollment in Degree-Granting Institutions by Race/Ethnicity Model, 1980-2009. (This table was prepared March 2011.) 
Table A-28. Estimated equations and model statistics for full-time and part-time college enrollment rates of Asian/Pacific Islander men

\begin{tabular}{|c|c|c|c|c|c|}
\hline Independent variable & Coefficient & Standard error & $t$-statistic & $R^{2}$ & D.W. statistic \\
\hline \multicolumn{6}{|l|}{ Full-time } \\
\hline Intercept term for 17 -year-olds & -9.28 & 0.338 & -14.87 & 0.94 & $1.89^{\star}$ \\
\hline Intercept term for 18 -year-olds & -6.47 & 0.305 & -10.11 & & \\
\hline Intercept term for 19 -year-olds & -6.27 & 0.309 & -9.69 & & \\
\hline Intercept term for 20 -year-olds & -6.37 & 0.306 & -9.94 & & \\
\hline Intercept term for 21-year-olds & -6.34 & 0.309 & -9.87 & & \\
\hline Intercept term for 22-year-olds & -6.70 & 0.307 & -10.48 & & \\
\hline Intercept term for 23-year-olds & -7.03 & 0.309 & -10.88 & & \\
\hline Intercept term for 24 -year-olds & -7.37 & 0.313 & -11.46 & & \\
\hline Intercept term for 25- to 29 -year-olds & -8.16 & 0.307 & -13.19 & & \\
\hline Intercept term for 30 - to 34 -year-olds & -9.19 & 0.309 & -14.98 & & \\
\hline Intercept term for 35 - to 44 -year-olds & -9.96 & 0.307 & -16.47 & & \\
\hline \multicolumn{5}{|l|}{ Log of Asian/Pacific Islander per capita } & \\
\hline \multicolumn{6}{|l|}{ Part-time } \\
\hline Intercept term for 17 -year-olds & -4.97 & 0.557 & -8.92 & 0.72 & $1.80^{*}$ \\
\hline Intercept term for 18 -year-olds & -3.94 & 0.552 & -7.13 & & \\
\hline Intercept term for 19 -year-olds & -3.21 & 0.544 & -5.90 & & \\
\hline Intercept term for 20 -year-olds & -3.32 & 0.555 & -5.99 & & \\
\hline Intercept term for 21-year-olds & -3.35 & 0.558 & -5.99 & & \\
\hline Intercept term for 22-year-olds & -3.38 & 0.570 & -5.93 & & \\
\hline Intercept term for 23-year-olds & -3.52 & 0.549 & -6.40 & & \\
\hline Intercept term for 24 -year-olds & -3.74 & 0.543 & -6.88 & & \\
\hline Intercept term for 25- to 29 -year-olds & -4.10 & 0.530 & -7.74 & & \\
\hline Intercept term for 30- to 34-year-olds & -4.55 & 0.531 & -8.58 & & \\
\hline Intercept term for 35 - to 44 -year-olds & -5.00 & 0.528 & -9.47 & & \\
\hline \multicolumn{6}{|l|}{ Log of Asian/Pacific Islander per capita } \\
\hline disposable income in current dollars & 0.09 & 0.027 & 3.34 & & \\
\hline
\end{tabular}

${ }^{*} p<.05$.

NOTE: $R^{2}$ = Coefficient of determination. D.W. statistic = Durbin-Watson statistic, a test for autocorrelation among regression residuals. For more details, see Johnston, J., and Dinardo, J. (1996). Econometric Methods, New York: McGraw-Hill. The regression method used to estimate the full-time and part-time equations was the pooled seemingly unrelated regression method. The time period used to estimate the equations is from 1989 to 2009. The number of observations is 231. For additional information, see Intriligator, M.D. (1978). Econometric Models, Techniques, \& Applications. New Jersey: Prentice-Hall, Inc., pp. 165-173. Race categories exclude persons of Hispanic ethnicity.

SOURCE: U.S. Department of Education, National Center for Education Statistics, Enrollment in Degree-Granting Institutions by Race/Ethnicity Model, 1980-2009. (This table was prepared March 2011.) 
Table A-29. Estimated equations and model statistics for full-time and part-time college enrollment rates of Asian/Pacific Islander women

\begin{tabular}{|c|c|c|c|c|c|}
\hline Independent variable & Coefficient & Standard error & $t$-statistic & $R^{2}$ & D.W. statistic \\
\hline \multicolumn{6}{|l|}{ Full-time } \\
\hline Intercept term for 17 -year-olds & -14.05 & 0.578 & -24.30 & 0.96 & $1.92^{*}$ \\
\hline Intercept term for 18-year-olds & -11.61 & 0.567 & -20.47 & & \\
\hline Intercept term for 19-year-olds & -11.03 & 0.572 & -19.27 & & \\
\hline Intercept term for 20-year-olds & -11.29 & 0.574 & -19.68 & & \\
\hline Intercept term for 21-year-olds & -11.41 & 0.569 & -20.04 & & \\
\hline Intercept term for 22-year-olds & -11.99 & 0.569 & -21.08 & & \\
\hline Intercept term for 23-year-olds & -12.40 & 0.568 & -21.84 & & \\
\hline Intercept term for 24-year-olds & -12.90 & 0.580 & -22.25 & & \\
\hline Intercept term for 25 - to 29 -year-olds & -13.81 & 0.565 & -24.45 & & \\
\hline Intercept term for 30- to 34-year-olds & -15.08 & 0.570 & -26.47 & & \\
\hline Intercept term for 35- to 44-year-olds & -15.63 & 0.572 & -27.31 & & \\
\hline \multicolumn{6}{|l|}{ Log of Asian/Pacific Islander per capita } \\
\hline disposable income in current dollars & 0.60 & 0.029 & 20.57 & & \\
\hline \multicolumn{6}{|l|}{ Part-time } \\
\hline Intercept term for 17 -year-olds & -13.00 & 0.483 & -26.92 & 0.86 & $1.89^{*}$ \\
\hline Intercept term for 18-year-olds & -11.11 & 0.465 & -23.89 & & \\
\hline Intercept term for 19-year-olds & -10.21 & 0.496 & -20.59 & & \\
\hline Intercept term for 20-year-olds & -10.58 & 0.476 & -22.20 & & \\
\hline Intercept term for 21-year-olds & -10.11 & 0.475 & -21.28 & & \\
\hline Intercept term for 22-year-olds & -10.41 & 0.454 & -22.92 & & \\
\hline Intercept term for 23-year-olds & -10.81 & 0.465 & -23.23 & & \\
\hline Intercept term for 24-year-olds & -10.98 & 0.480 & -22.88 & & \\
\hline Intercept term for 25 - to 29 -year-olds & -11.47 & 0.449 & -25.52 & & \\
\hline Intercept term for 30- to 34-year-olds & -12.12 & 0.452 & -26.80 & & \\
\hline Intercept term for 35- to 44-year-olds & -11.97 & 0.446 & -26.80 & & \\
\hline \multicolumn{6}{|l|}{ Log of Asian/Pacific Islander per capita } \\
\hline disposable income in current dollars & 0.47 & 0.023 & 20.65 & & \\
\hline
\end{tabular}

${ }^{*} p<.05$.

NOTE: $R^{2}=$ Coefficient of determination. D.W. statistic = Durbin-Watson statistic, a test for autocorrelation among regression residuals. For more details, see Johnston, J., and Dinardo, J. (1996). Econometric Methods, New York: McGraw-Hill. The regression method used to estimate the full-time and part-time equations was the pooled seemingly unrelated regression method. The time period used to estimate the equations is from 1989 to 2009. The number of observations is 231. For additional information, see Intriligator, M.D. (1978). Econometric Models, Techniques, \& Applications. New Jersey: Prentice-Hall, Inc., pp. 165-173. Race categories exclude persons of Hispanic ethnicity.

SOURCE: U.S. Department of Education, National Center for Education Statistics, Enrollment in Degree-Granting Institutions by Race/Ethnicity Model, 1980-2009. (This table was prepared March 2011.) 


\section{Projections in this edition}

This edition of Projections of Education Statistics presents projections of postsecondary degrees conferred by level of degree and sex of recipient for 2009-10 through 2020-21.

\section{Overview of approach}

\section{Basic approach}

Projections of associate's, bachelor's, master's, and first-professional degrees for men and women were produced using forecasting equations that relate degrees conferred to full-time enrollment in degree-granting institutions by sex, student level (undergraduate or postbaccalaureate), and institution level (2-year or 4-year). Projections of doctor's degrees for men and women were produced using double exponential smoothing.

\section{Degrees Conferred Model}

\section{Procedures used to project degrees}

For all degree levels, projections of degrees conferred were made separately for men and for women. The projections for men and women were then summed to get projections of the total number of degrees.

Double exponential smoothing was used to project doctor's degrees, as described below. Multiple linear regression was used to project associate's, bachelor's, master's, and first-professional degrees based on enrollment variables for men and women. The enrollment variables used for the different levels of degrees are briefly described below.

For details and results of the regression analyses used to project associate's, bachelor's, master's, and first-professional degrees, see table A-30, under "Data and equations used to project degrees," later in this section.

Associate's degrees. Projections were based on full-time undergraduate enrollment in 2-year institutions by sex. Men's projections of associate's degrees were based on current full-time enrollment and full-time enrollment lagged 2 years. Women's projections of associate's degrees were based on current full-time enrollment and full-time enrollment lagged 1 and 2 years.

Bachelor's degrees. Projections were based on full-time undergraduate enrollment in 4-year institutions by sex. For men and for women, bachelor's degree projections were based on current full-time enrollment and full-time enrollment lagged 2 years.

Master's degrees. Projections were based on full-time postbaccalaureate enrollment by sex. Men's projections of master's degrees were based on current full-time enrollment and full-time enrollment lagged 1 year. Women's projections of master's degrees were based on current full-time enrollment.

Doctor's degrees. Projections were obtained by double exponential smoothing of the historical data on doctor's degrees awarded by sex. The smoothing constants for each sex were chosen to minimize the sum of squared forecast errors.

First-professional degrees. Projections were based on full-time postbaccalaureate enrollment by sex. For men and for women, first-professional degree projections were based on current full-time postbaccalaureate enrollment and full-time postbaccalaureate enrollment lagged 1 and 2 years.

\section{Data and equations used to project degrees}

Enrollment data and projections for degree-granting institutions. Historical enrollment data by sex, level of student, and level of institution came from the NCES Integrated Postsecondary Education Data System (IPEDS). For the time period used for each level of degree, see table A-30 on page 137. The enrollment projections used are those produced for this edition of Projections of Education Statistics. For more information about the enrollment projections, see Section A.5. Enrollment in Postsecondary Degree-Granting Institutions, earlier in this appendix.

Data on degrees awarded at all levels. Historical data by level of degree and sex of recipient came from the NCES Integrated Postsecondary Education Data System (IPEDS). Doctor's degrees were projected using data for 1960-61 to 2007-08. For the time periods used for the other degree levels, see table A-30 on page 137. 
Estimated equations and model statistics. For details on the equations used to project associate's, bachelor's, master's, and first-professional degrees, see table A-30 on page 137. The equations shown were selected on the basis of their statistical properties, such as coefficients of determination $\left(R^{2} s\right)$, the $t$-statistics of the coefficients, the Durbin-Watson statistic, the Breusch-Godfrey Serial Correlation LM test statistic, and residual plots.

\section{Accuracy of projections}

No MAPEs were calculated for degrees conferred as the current models used for producing these projections have been used for only two other editions of Projections of Education Statistics.

For more information about MAPEs, see Section A.O. Introduction, earlier in this appendix. 


\begin{tabular}{|c|c|c|c|c|c|c|c|c|c|}
\hline Dependent variable & & & Equation $^{1}$ & & & & $R^{2}$ & $\begin{array}{r}\text { Breusch- } \\
\text { Godfrey } \\
\text { Serial } \\
\text { Correlation } \\
\text { LM test } \\
\text { statistic }^{2}\end{array}$ & $\begin{array}{r}\text { Time } \\
\text { period } \\
\end{array}$ \\
\hline Associate's degrees, men & DASSOCM = & $\begin{aligned}= & 1,907+ \\
& (2.3)\end{aligned}$ & $\begin{array}{r}\text { 89DUGFT2M } \\
(4.4)\end{array}$ & + & $\begin{array}{r}\text { 58DUGFT2ML2 } \\
(3.0)\end{array}$ & & 0.51 & $0.13(0.951)$ & $\begin{array}{r}1980-81 \text { to } \\
2008-09\end{array}$ \\
\hline Associate's degrees, women & DLOGASSOCW = & $\begin{array}{l}\#+ \\
(\dagger)\end{array}$ & $\begin{array}{r}0.7 D L O G U G F T 2 W S 3 \\
(3.9)\end{array}$ & + & $\begin{array}{r}0.5 \mathrm{MA}(1) \\
(2.9)\end{array}$ & & 0.59 & $0.02(0.992)$ & $\begin{array}{r}1980-81 \text { to } \\
2008-09\end{array}$ \\
\hline Bachelor's degrees, men & DBACHM = & $=\underset{(0.70)}{667}+$ & $\begin{array}{r}\text { 82DUGFT4M } \\
(3.6)\end{array}$ & + & $\begin{array}{r}\text { 141DUGFT4ML2 } \\
(5.8)\end{array}$ & & 0.81 & $1.462(0.445)$ & $\begin{array}{r}1980-81 \text { to } \\
2008-09\end{array}$ \\
\hline Bachelor's degrees, women & DBACHW = & $\begin{array}{c}=3772+ \\
(1.7)\end{array}$ & $\begin{array}{r}\text { 87DUGFT4W } \\
(2.8)\end{array}$ & + & $\begin{array}{r}\text { 108DUGFT4WL2 } \\
(3.2)\end{array}$ & & 0.62 & $0.76(0.684)$ & $\begin{array}{r}1980-81 \text { to } \\
2008-09\end{array}$ \\
\hline Master's degrees, men & PCHMASTM = & $\begin{array}{r}\#+ \\
(\dagger)\end{array}$ & $\begin{array}{r}0.6 \mathrm{PCHPBFTM} \\
(4.38)\end{array}$ & + & $\begin{array}{r}0.5 P C H P B F T M L 1 \\
(3.71)\end{array}$ & $\begin{array}{r}0.4 \mathrm{AR}(1) \\
(2.66)\end{array}$ & 0.67 & $2.98(0.226)$ & $\begin{array}{r}1970-71 \text { to } \\
2008-09\end{array}$ \\
\hline Master's degrees, women & PCHMASTW $=$ & $\begin{array}{l}\#+ \\
(\dagger)\end{array}$ & $\begin{array}{r}0.4 \mathrm{PCHPBFTW} \\
(2.35)\end{array}$ & + & $\begin{array}{r}0.6 \mathrm{AR}(1) \\
(3.79)\end{array}$ & & 0.60 & $1.13(0.579)$ & $\begin{array}{r}1970-71 \text { to } \\
2008-09\end{array}$ \\
\hline $\begin{array}{l}\text { First-professional degrees, } \\
\text { men }\end{array}$ & DFPROM = & $=\begin{array}{r}91+ \\
(0.5)\end{array}$ & $\begin{array}{r}\text { 162DPBFTML1 } \\
(5.2)\end{array}$ & + & $\begin{array}{r}\text { 89DPBFTML2 } \\
(3.0)\end{array}$ & & 0.61 & $3.34(0.188)$ & $\begin{array}{r}1971-72 \text { to } \\
2008-09\end{array}$ \\
\hline $\begin{array}{l}\text { First-professional degrees, } \\
\text { women }\end{array}$ & DFPROW = & $=\begin{array}{r}44+ \\
(0.2)\end{array}$ & $\begin{array}{r}\text { 123DPBFTWL1 } \\
(2.5)\end{array}$ & + & $\begin{array}{r}\text { 165DPBFTWL2 } \\
(3.8)\end{array}$ & & 0.51 & $2.97(0.23)$ & $\begin{array}{r}1971-72 \text { to } \\
2008-09\end{array}$ \\
\hline
\end{tabular}

\# Rounds to zero.

† Not applicable.

${ }^{1} \mathrm{AR}(1)$ indicates that the model was estimated to account for first-order autocorrelation. To estimate the model, it was first transformed into a nonlinear model and then the coefficients were estimated simultaneously by applying a Marquardt nonlinear least squares algorithm to the transformed equation. MA(1) indicates that the model was estimated to incorporate moving average of the residual into model fit. For a general discussion of the problem of autocorrelation and the method used to forecast in the presence of autocorrelation, see Judge, G., Hill, W., Griffiths, R., Lutkepohl, H., and Lee, T. (1985). The Theory and Practice of Econometrics, New York: John Wiley and Sons, pp. 315-318.

2 The number in parentheses is the probability of the Chi-Square(2) associated with the Breusch-Godfrey Serial Correlation LM Test. A $p$ value greater than 0.05 implies that we do not reject the null hypothesis of no autocorrelation at the 5 percent significance level for a two-tailed test and the 10 percent significance level for a one-tailed test (i.e., there is no autocorrelation present). For an explanation of the Breusch-Godfrey Serial Correlation LM test statistic, see Greene, W. (2000). Econometric Analysis. New Jersey: Prentice-Hall.

NOTE: $R^{2}$ is the coefficient of determination. Numbers in parentheses are $t$-statistics. There are no equations for doctor's degrees for men and women as projections of those items were obtained using double exponential smoothing.

DASSOCM = First difference of associate's degrees awarded to men.

DLOGASSOCW $=$ First difference of the log of associate's degrees awarded to women.

DBACHM = First difference of bachelor's degrees awarded to men.

DBACHW = First difference of bachelor's degrees awarded to women.

PCHMASTM = Percentage change in master's degrees awarded to men.

PCHMASTW $=$ Percentage change in master's degrees awarded to women

DFPROM = First difference of first-professional degrees awarded to men.

DFPROW = First difference of first-professional degrees awarded to women.

DUGFT2M = First difference of full-time male undergraduate enrollment in 2-year institutions.

DUGFT2ML2 = First difference of full-time male undergraduate enrollment in 2-year institutions, lagged two periods.

DLOGUGFT2WS3 = First difference of the sum of the full-time female undergraduate enrollment in 2-year institutions over the present year and the previous 2 years.

DUGFT4M = First difference of full-time male undergraduate enrollment in 4-year institutions.

DUGFT4ML2 = First difference of full-time male undergraduate enrollment in 4-year institutions, lagged two periods.

DUGFT4W = First difference of full-time female undergraduate enrollment in 4-year institutions.

DUGFT4WL2 = First difference of full-time female undergraduate enrollment in 4-year institutions, lagged two periods.

PCHPBFTM = Percentage change in full-time male postbaccalaureate enrollment.

PCHPBFTML1 = Percentage change in full-time female postbaccalaureate enrollment lagged one year.

PCHPBFTW = Percentage change in full-time female postbaccalaureate enrollment.

DBPFTML1 = First difference of full-time male postbaccalaureate enrollment lagged one year.

DBPFTML2 = First difference of full-time male postbaccalaureate enrollment lagged two years.

DBPFTWL1 = First difference of full-time female postbaccalaureate enrollment lagged one year.

DBPFTWL2 = First difference of full-time female postbaccalaureate enrollment lagged two years.

SOURCE: U.S. Department of Education, National Center for Education Statistics, Degrees Conferred Model, 1970-71 through 2008-09. (This table was prepared March 2010.) 
This page intentionally left blank. 


\section{Appendix B Supplementary Tables}


Table B-1. Annual number of births: 1946 through 2009

\begin{tabular}{|c|c|c|c|}
\hline Calendar year & $\begin{array}{l}\text { Number } \\
\text { of births, in } \\
\text { thousands }\end{array}$ & Calendar year & $\begin{array}{l}\text { Number } \\
\text { of births, in } \\
\text { thousands }\end{array}$ \\
\hline 1946 & 3,426 & 1978 & 3,333 \\
\hline 1947 & 3,834 & 1979 & 3,494 \\
\hline 1948 & 3,655 & 1980 & 3,612 \\
\hline 1949 & 3,667 & 1981 & 3,629 \\
\hline 1950 & 3,645 & 1982 & 3,681 \\
\hline 1951 & 3,845 & 1983 & 3,639 \\
\hline 1952 & 3,933 & 1984 & 3,669 \\
\hline 1953 & 3,989 & 1985 & 3,761 \\
\hline 1954 & 4,102 & 1986 & 3,757 \\
\hline 1955 & 4,128 & 1987 & 3,809 \\
\hline 1956 & 4,244 & 1988 & 3,910 \\
\hline 1957 & 4,332 & 1989 & 4,041 \\
\hline 1958 & 4,279 & 1990 & 4,158 \\
\hline 1959 & 4,313 & 1991 & 4,111 \\
\hline 1960 & 4,307 & 1992 & 4,065 \\
\hline 1961 & 4,317 & 1993 & 4,000 \\
\hline 1962 & 4,213 & 1994 & 3,953 \\
\hline 1963 & 4,142 & 1995 & 3,900 \\
\hline 1964 & 4,070 & 1996 & 3,891 \\
\hline 1965 & 3,801 & 1997 & 3,881 \\
\hline 1966 & 3,642 & 1998 & 3,942 \\
\hline 1967 & 3,555 & 1999 & 3,959 \\
\hline 1968 & 3,535 & 2000 & 4,059 \\
\hline 1969 & 3,626 & 2001 & 4,026 \\
\hline 1970 & 3,739 & 2002 & 4,022 \\
\hline 1971 & 3,556 & 2003 & 4,090 \\
\hline 1972 & 3,258 & 2004 & 4,112 \\
\hline 1973 & 3,137 & 2005 & 4,138 \\
\hline 1974 & 3,160 & 2006 & 4,266 \\
\hline 1975 & 3,144 & 2007 & 4,317 \\
\hline 1976 & 3,168 & 2008 & 4,248 \\
\hline 1977 & 3,327 & 2009 & 4,131 \\
\hline
\end{tabular}

NOTE: Some data have been revised from previously published figures.

SOURCE: U.S. Department of Health and Human Services, National Center for Health Statistics (NCHS), National Vital Statistics Reports, various years. (This table was prepared February 2011.) 
Table B-2. Actual and projected prekindergarten- and kindergarten-age populations, by age: 1995 through 2020 [In thousands]

\begin{tabular}{|c|c|c|c|c|}
\hline Year (July 1) & 3- to 5-year-olds & 3-year-olds & 4-year-olds & 5-year-olds \\
\hline \multicolumn{5}{|l|}{ Actual } \\
\hline 1995 & 12,188 & 4,004 & 4,103 & 4,081 \\
\hline 1996 & 12,141 & 3,936 & 4,086 & 4,119 \\
\hline 1997 & 12,019 & 3,894 & 4,021 & 4,104 \\
\hline 1998 & 11,880 & 3,862 & 3,979 & 4,040 \\
\hline 1999 & 11,768 & 3,827 & 3,946 & 3,996 \\
\hline 2000 & 11,703 & 3,826 & 3,906 & 3,971 \\
\hline 2001 & 11,601 & 3,828 & 3,847 & 3,926 \\
\hline 2002 & 11,563 & 3,850 & 3,847 & 3,865 \\
\hline 2003 & 11,657 & 3,928 & 3,867 & 3,863 \\
\hline 2004 & 11,921 & 4,098 & 3,942 & 3,881 \\
\hline 2005 & 12,122 & 4,052 & 4,113 & 3,957 \\
\hline 2006 & 12,292 & 4,097 & 4,067 & 4,128 \\
\hline 2007 & 12,356 & 4,159 & 4,113 & 4,084 \\
\hline 2008 & 12,468 & 4,168 & 4,173 & 4,127 \\
\hline 2009 & 12,590 & 4,224 & 4,181 & 4,186 \\
\hline \multicolumn{5}{|l|}{ Projected } \\
\hline 2010 & 12,779 & 4,349 & 4,236 & 4,193 \\
\hline 2011 & 12,874 & 4,386 & 4,268 & 4,220 \\
\hline 2012 & 12,983 & 4,428 & 4,304 & 4,251 \\
\hline 2013 & 13,104 & 4,471 & 4,345 & 4,288 \\
\hline 2014 & 13,231 & 4,515 & 4,388 & 4,328 \\
\hline 2015 & 13,358 & 4,557 & 4,430 & 4,371 \\
\hline 2016 & 13,481 & 4,596 & 4,471 & 4,414 \\
\hline 2017 & 13,597 & 4,632 & 4,510 & 4,455 \\
\hline 2018 & 13,705 & 4,665 & 4,546 & 4,494 \\
\hline 2019 & 13,804 & 4,696 & 4,578 & 4,529 \\
\hline 2020 & 13,896 & 4,725 & 4,609 & 4,562 \\
\hline
\end{tabular}

NOTE: Some data have been revised from previously published figures. Detail may not sum to totals because of rounding. Projections are from the U.S. Census Bureau's 2008 National Population Projections, ratio-adjusted to line up with the most recent historical estimate.

SOURCE: U.S. Department of Commerce, Census Bureau, Population Estimates, retrieved October 4, 2010, from http://www.census.gov/popest/ national/; and Population Projections, retrieved November 2, 2009, from http://www.census.gov/population/www/projections/2008projections.html. (This table was prepared January 2011.) 
Table B-3. Actual and projected school-age populations, by selected ages: 1995 through 2020 [In thousands]

\begin{tabular}{|c|c|c|c|c|}
\hline Year (July 1) & 5-year-olds & 6-year-olds & $\begin{array}{l}5 \text { - to } 13- \\
\text { year-olds }\end{array}$ & $\begin{array}{l}14-\text { to } 17- \\
\text { year-olds }\end{array}$ \\
\hline \multicolumn{5}{|l|}{ Actual } \\
\hline 1995 & 4,081 & 3,919 & 34,825 & 15,013 \\
\hline 1996 & 4,119 & 4,088 & 35,375 & 15,443 \\
\hline 1997 & 4,104 & 4,127 & 35,915 & 15,769 \\
\hline 1998 & 4,040 & 4,112 & 36,454 & 15,829 \\
\hline 1999 & 3,996 & 4,045 & 36,804 & 16,007 \\
\hline 2000 & 3,971 & 4,008 & 37,051 & 16,131 \\
\hline 2001 & 3,926 & 3,990 & 37,085 & 16,221 \\
\hline 2002 & 3,865 & 3,944 & 36,980 & 16,401 \\
\hline 2003 & 3,863 & 3,881 & 36,774 & 16,544 \\
\hline 2004 & 3,881 & 3,877 & 36,396 & 16,854 \\
\hline 2005 & 3,957 & 3,895 & 36,162 & 17,104 \\
\hline 2006 & 4,128 & 3,971 & 36,159 & 17,239 \\
\hline 2007 & 4,084 & 4,144 & 36,180 & 17,239 \\
\hline 2008 & 4,127 & 4,097 & 36,297 & 16,980 \\
\hline 2009 & 4,186 & 4,139 & 36,487 & 16,761 \\
\hline \multicolumn{5}{|l|}{ Projected } \\
\hline 2010 & 4,193 & 4,198 & 36,736 & 16,550 \\
\hline 2011 & 4,220 & 4,226 & 37,103 & 16,397 \\
\hline 2012 & 4,251 & 4,253 & 37,504 & 16,306 \\
\hline 2013 & 4,288 & 4,285 & 37,902 & 16,283 \\
\hline 2014 & 4,328 & 4,322 & 38,302 & 16,327 \\
\hline 2015 & 4,371 & 4,364 & 38,600 & 16,574 \\
\hline 2016 & 4,414 & 4,407 & 38,941 & 16,822 \\
\hline 2017 & 4,455 & 4,451 & 39,297 & 17,057 \\
\hline 2018 & 4,494 & 4,492 & 39,645 & 17,300 \\
\hline 2019 & 4,529 & 4,531 & 40,001 & 17,424 \\
\hline 2020 & 4,562 & 4,568 & 40,362 & 17,576 \\
\hline
\end{tabular}

NOTE: Some data have been revised from previously published figures. Projections are from the U.S. Census Bureau's 2008 National Population Projections, ratio-adjusted to line up with the most recent historical estimate.

SOURCE: U.S. Department of Commerce, Census Bureau, Population Estimates, retrieved October 4, 2010, from http://www.census.gov/popest/ national/; and Population Projections, retrieved November 2, 2009, from http://www.census.gov/population/www/projections/

2008projections.html. (This table was prepared January 2011.) 
Table B-4. Actual and projected college-age populations, by selected ages: 1995 through 2020

[In thousands]

\begin{tabular}{|c|c|c|c|c|c|}
\hline Year (July 1) & 18-year-olds & $\begin{array}{l}18 \text { - to } 24- \\
\text { year-olds }\end{array}$ & $\begin{array}{r}25-\text { to } 29- \\
\text { year-olds }\end{array}$ & $\begin{array}{l}30 \text { - to } 34- \\
\text { year-olds }\end{array}$ & $\begin{array}{l}35-\text { to } 44- \\
\text { year-olds }\end{array}$ \\
\hline \multicolumn{6}{|l|}{ Actual } \\
\hline 1995 & 3,601 & 25,585 & 19,742 & 22,425 & 42,765 \\
\hline 1996 & 3,650 & 25,376 & 19,927 & 21,996 & 43,605 \\
\hline 1997 & 3,780 & 25,574 & 19,960 & 21,494 & 44,282 \\
\hline 1998 & 3,984 & 26,155 & 19,863 & 20,999 & 44,802 \\
\hline 1999 & 3,993 & 26,780 & 19,632 & 20,647 & 45,130 \\
\hline 2000 & 4,078 & 27,391 & 19,343 & 20,566 & 45,217 \\
\hline 2001 & 4,087 & 28,089 & 18,945 & 20,722 & 45,114 \\
\hline 2002 & 4,052 & 28,606 & 18,891 & 20,763 & 44,729 \\
\hline 2003 & 4,154 & 29,117 & 19,071 & 20,624 & 44,284 \\
\hline 2004 & 4,150 & 29,458 & 19,458 & 20,320 & 43,920 \\
\hline 2005 & 4,146 & 29,572 & 19,960 & 19,910 & 43,633 \\
\hline 2006 & 4,205 & 29,696 & 20,601 & 19,490 & 43,361 \\
\hline 2007 & 4,285 & 29,899 & 21,116 & 19,413 & 42,934 \\
\hline 2008 & 4,459 & 30,251 & 21,542 & 19,576 & 42,324 \\
\hline 2009 & 4,391 & 30,569 & 21,783 & 19,951 & 41,615 \\
\hline \multicolumn{6}{|l|}{ Projected } \\
\hline 2010 & 4,332 & 30,729 & 21,888 & 20,432 & 41,107 \\
\hline 2011 & 4,291 & 30,972 & 21,936 & 21,012 & 40,890 \\
\hline 2012 & 4,233 & 31,147 & 22,034 & 21,482 & 40,856 \\
\hline 2013 & 4,212 & 31,215 & 22,217 & 21,846 & 40,932 \\
\hline 2014 & 4,191 & 31,166 & 22,515 & 22,058 & 41,039 \\
\hline 2015 & 4,142 & 30,920 & 22,891 & 22,135 & 41,132 \\
\hline 2016 & 4,147 & 30,677 & 23,227 & 22,188 & 41,358 \\
\hline 2017 & 4,194 & 30,534 & 23,499 & 22,291 & 41,756 \\
\hline 2018 & 4,241 & 30,481 & 23,642 & 22,477 & 42,310 \\
\hline 2019 & 4,395 & 30,643 & 23,629 & 22,774 & 42,911 \\
\hline 2020 & 4,402 & 30,833 & 23,451 & 23,149 & 43,485 \\
\hline
\end{tabular}

NOTE: Some data have been revised from previously published figures. Projections are from the U.S. Census Bureau's 2008 National Population Projections, ratio-adjusted to line up with the most recent historical estimate.

SOURCE: U.S. Department of Commerce, Census Bureau, Population Estimates, retrieved October 4, 2010, from http://www.census.gov/popest/ national/; and Population Projections, retrieved November 2, 2009, from http://www.census.gov/population/www/projections/

2008projections.html. (This table was prepared January 2011.) 
Table B-5. Actual and projected fall enrollment in public elementary and secondary schools, change in fall enrollment from previous year, resident population, and fall enrollment as a ratio of the population: School years 1995-96 through 2020-21

\begin{tabular}{|c|c|c|c|c|}
\hline School year & $\begin{array}{l}\text { Fall enrollment } \\
\text { (in thousands) }\end{array}$ & $\begin{array}{r}\text { Change in fall enrollment } \\
\text { from previous year } \\
\text { (in thousands) }\end{array}$ & $\begin{array}{r}\text { Resident } \\
\text { population } \\
\text { (in millions) } \\
\end{array}$ & $\begin{array}{r}\text { Fall enrollment } \\
\text { as a ratio of } \\
\text { the population }\end{array}$ \\
\hline \multicolumn{5}{|l|}{ Actual } \\
\hline 1995-96 & 44,840 & 729 & 266.6 & 0.168 \\
\hline 1996-97 & 45,611 & 771 & 269.7 & 0.169 \\
\hline $1997-98$ & 46,127 & 516 & 272.9 & 0.169 \\
\hline 1998-99 & 46,539 & 412 & 276.1 & 0.169 \\
\hline 1999-2000 & 46,857 & 319 & 279.3 & 0.168 \\
\hline $2000-01$ & 47,204 & 346 & 282.4 & 0.167 \\
\hline 2001-02 & 47,672 & 468 & 285.3 & 0.167 \\
\hline $2002-03$ & 48,183 & 511 & 288.1 & 0.167 \\
\hline 2003-04 & 48,540 & 357 & 290.8 & 0.167 \\
\hline 2004-05 & 48,795 & 255 & 293.5 & 0.166 \\
\hline $2005-06$ & 49,113 & 318 & 296.2 & 0.166 \\
\hline $2006-07$ & 49,316 & 203 & 299.0 & 0.165 \\
\hline $2007-08$ & 49,293 & -23 & 302.0 & 0.163 \\
\hline 2008-09 & 49,265 & -27 & 304.8 & 0.162 \\
\hline \multicolumn{5}{|l|}{ Projected } \\
\hline $2009-10$ & 49,282 & 17 & 307.4 & 0.160 \\
\hline 2010-11 & 49,306 & 24 & 310.1 & 0.159 \\
\hline $2011-12$ & 49,422 & 116 & 313.1 & 0.158 \\
\hline $2012-13$ & 49,642 & 220 & 316.1 & 0.157 \\
\hline 2013-14 & 49,914 & 272 & 319.2 & 0.156 \\
\hline 2014-15 & 50,268 & 354 & 322.2 & 0.156 \\
\hline $2015-16$ & 50,659 & 392 & 325.4 & 0.156 \\
\hline $2016-17$ & 51,038 & 378 & 328.5 & 0.155 \\
\hline $2017-18$ & 51,430 & 392 & 331.7 & 0.155 \\
\hline 2018-19 & 51,803 & 373 & 334.8 & 0.155 \\
\hline $2019-20$ & 52,204 & 401 & 338.0 & 0.154 \\
\hline $2020-21$ & 52,666 & 462 & 341.2 & 0.154 \\
\hline
\end{tabular}

NOTE: Resident population includes civilian population and armed forces personnel residing within the United States; it excludes armed forces personnel overseas. Calculations were made using unrounded numbers. Some data have been revised from previously published figures. Projections are from the U.S. Census Bureau's 2008 National Population Projections, ratio-adjusted to line up with the most recent historical estimate.

SOURCE: U.S. Department of Commerce, Census Bureau, Population Estimates, retrieved October 4, 2010, from http://www.census.gov/popest/ national/; and Population Projections, retrieved November 2, 2009, from http://www.census.gov/population/www/projections/2008projections.html. U.S. Department of Education, National Center for Education Statistics, Common Core of Data (CCD), "State Nonfiscal Survey of Public Elementary/ Secondary Education," 1995-96 through 2008-09; and Elementary and Secondary Enrollment Model, 1972-2008. (This table was prepared March 2011.) 
Table B-6. Actual and projected macroeconomic measures of the economy: School years 1995-96 through 2020-21

\begin{tabular}{|c|c|c|c|}
\hline School year & $\begin{array}{r}\text { Disposable income } \\
\text { per capita in constant } \\
2008-09 \text { dollars } 1\end{array}$ & $\begin{array}{r}\text { Education revenue receipts } \\
\text { from state sources } \\
\text { per capita in constant } \\
2008-09 \text { dollars }^{2}\end{array}$ & $\begin{array}{l}\text { Consumer } \\
\text { Price Index }\end{array}$ \\
\hline \multicolumn{4}{|l|}{ Actual } \\
\hline 1995-96 & $\$ 27,616$ & $\$ 712$ & 0.720 \\
\hline $1996-97$ & 28,233 & 733 & 0.740 \\
\hline 1997-98 & 29,272 & 766 & 0.754 \\
\hline 1998-99 & 30,265 & 800 & 0.767 \\
\hline 1999-2000 & 31,083 & 838 & 0.789 \\
\hline $2000-01$ & 31,886 & 866 & 0.816 \\
\hline 2001-02 & 32,623 & 872 & 0.830 \\
\hline $2002-03$ & 32,905 & 877 & 0.849 \\
\hline 2003-04 & 33,807 & 862 & 0.867 \\
\hline 2004-05 & 34,300 & 872 & 0.893 \\
\hline 2005-06 & 34,810 & 882 & 0.927 \\
\hline $2006-07$ & 35,679 & 927 & 0.951 \\
\hline $2007-08$ & 36,094 & 949 & 0.986 \\
\hline 2008-09 & 35,999 & 934 & 1.000 \\
\hline \multicolumn{4}{|l|}{ Projected } \\
\hline $2009-10^{4}$ & 35,911 & 924 & 1.010 \\
\hline $2010-11$ & 36,150 & 925 & 1.023 \\
\hline $2011-12$ & 36,201 & 921 & 1.041 \\
\hline $2012-13$ & 36,250 & 919 & 1.062 \\
\hline 2013-14 & 36,626 & 926 & 1.084 \\
\hline 2014-15 & 37,576 & 951 & 1.108 \\
\hline $2015-16$ & 38,490 & 976 & 1.132 \\
\hline 2016-17 & 39,375 & 1,000 & 1.156 \\
\hline $2017-18$ & 40,116 & 1,019 & 1.180 \\
\hline 2018-19 & 40,843 & 1,038 & 1.205 \\
\hline $2019-20$ & 41,647 & 1,060 & 1.228 \\
\hline $2020-21$ & 42,218 & 1,075 & 1.246 \\
\hline
\end{tabular}

${ }^{1}$ Based on the price deflator for personal consumption expenditures, Bureau of Labor Statistics, U.S. Department of Labor.

${ }^{2}$ Based on the Consumer Price Index for all urban consumers, Bureau of Labor Statistics, U.S. Department of Labor.

${ }^{3}$ Consumer Price Index adjusted to a school-year basis (July through June).

${ }^{4}$ Disposable income per capita and consumer price index numbers are actual numbers.

NOTE: Calculations were made using unrounded numbers. Some data have been revised from previously published figures.

SOURCE: U.S. Department of Education, National Center for Education Statistics, Common Core of Data (CCD), "National Public Education

Financial Survey," 1995-96 through 2007-08; Revenue Receipts From State Sources Model, 1971-72 through 2007-08; and IHS Global Insight,

"U.S. Monthly Model: November 2010 Short-Term-Projections." (This table was prepared January 2011.) 
This page intentionally left blank. 


\section{Appendix C Data Sources}

\section{SOURCES AND COMPARABILITY OF DATA}

The information in this report was obtained from many sources, including federal and state agencies, private research organizations, and professional associations. The data were collected by many methods, including surveys of a universe (such as all colleges) or of a sample, and compilations of administrative records. Care should be used when comparing data from different sources. Differences in procedures, such as timing, phrasing of questions, and interviewer training, mean that the results from the different sources are not strictly comparable. More extensive documentation of one survey's procedures than of another's does not imply more problems with the data, only that more information is available on the survey.

\section{ACCURACY OF DATA}

The accuracy of any statistic is determined by the joint effects of "sampling" and "nonsampling" errors. Estimates based on a sample will differ from the figures that would have been obtained if a complete census had been taken using the same survey instruments, instructions, and procedures. Besides sampling errors, both of the surveys, universe and sample, are subject to errors of design, reporting, and processing, and errors due to nonresponse. To the extent possible, these nonsampling errors are kept to a minimum by methods built into the survey procedures. In general, however, the effects of nonsampling errors are more difficult to gauge than those produced by sampling variability.

\section{SAMPLING ERRORS}

The standard error is the primary measure of the sampling variability of an estimate. Standard errors can be used to produce confidence intervals. For example, from table A-12, an estimated 91.8 percent of public school teachers reported that they worked full time in 2007-08. This figure has an estimated standard error of 0.29 percent. Therefore, the estimated 95 percent confidence interval for this statistic is approximately 91.27 to 92.41 percent $(91.8 \pm 1.96(0.29))$. That is, if the processes of selecting a sample, collecting the data, and constructing the confidence interval were repeated, it would be expected that in 95 out of 100 samples from the same population, the confidence interval would contain the true full time working rate.

Analysis of standard errors can help assess how valid a comparison between two estimates might be. The standard error of a difference between two independent sample estimates is equal to the square root of the sum of the squared standard errors of the estimates. The standard error $(s e)$ of the difference between independent sample estimates $a$ and $b$ is

$$
s e_{a-b}=\left(s e_{a}^{2}+s e_{b}^{2}\right)^{1 / 2}
$$

Note that most of the standard errors in the original documents are approximations. That is, to derive estimates of standard errors that would be applicable to a wide variety of items and could be prepared at a moderate cost, a number of approximations were required. As a result, most of the standard errors presented provide a general order of magnitude rather than the exact standard error for any specific item.

\section{NONSAMPLING ERRORS}

Both universe and sample surveys are subject to nonsampling errors. Nonsampling errors are of two kinds—random and nonrandom. Random nonsampling errors may arise when respondents or interviewers interpret questions differently, when respondents must estimate values, or when coders, keyers, and other processors handle answers differently. Nonrandom nonsampling errors result from total nonresponse (no usable data obtained for a sampled unit), partial or item nonresponse (only a portion of a response may be usable), inability or unwillingness on the part of respondents to provide information, difficulty interpreting questions, mistakes in recording or keying data, errors of collection or processing, and overcoverage 
or undercoverage of the target universe. Random nonresponse errors usually, but not always, result in an understatement of sampling errors and thus an overstatement of the precision of survey estimates. Because estimating the magnitude of nonsampling errors would require special experiments or access to independent data, these magnitudes are seldom available.

To compensate for suspected nonrandom errors, adjustments of the sample estimates are often made. For example, adjustments are frequently made for nonresponse, both total and partial. Imputations are usually made separately within various groups of sample members that have similar survey characteristics. Imputation for item nonresponse is usually made by substituting for a missing item the response to that item of a respondent having characteristics similar to those of the respondent.

Although the magnitude of nonsampling errors in the data used in Projections of Education Statistics is frequently unknown, idiosyncrasies that have been identified are noted on the appropriate tables.

\section{FEDERAL AGENCY SOURCES}

\section{National Center for Education Statistics (NCES)}

\section{Common Core of Data}

NCES uses the Common Core of Data (CCD) to acquire and maintain statistical data from each of the 50 states, the District of Columbia, the Bureau of Indian Education, Department of Defense Dependents' Schools (overseas), and the outlying areas (American Samoa, Guam, Northern Marianas, Puerto Rico, and U.S. Virgin Islands). Information about staff and students is collected annually at the school, local education agency (LEA) or school district, and state levels. Information about revenues and expenditures is also collected at the state and LEA levels.

Data are collected for a particular school year via an on-line reporting system open to state education agencies during the school year. Since the CCD is a universe collection, CCD data are not subject to sampling errors. However, nonsampling errors could come from two sources: nonresponse and inaccurate reporting. Almost all of the states submit the five CCD survey instruments each year, but submissions are sometimes incomplete.

Misreporting can occur when 58 education agencies compile and submit data for approximately 100,000 public schools and over 18,000 local education agencies. Typically, this results from varying interpretations of NCES definitions and differing record-keeping systems. NCES attempts to minimize these errors by working closely with the state education agencies through the National Forum on Education Statistics.

The state education agencies report data to NCES from data collected and edited in their regular reporting cycles. NCES encourages the agencies to incorporate into their own survey systems the NCES items they do not already collect so that these items will also be available for the subsequent CCD survey. Over time, this has meant fewer missing data cells in each state's response, reducing the need to impute data.

NCES subjects data from the state education agencies to a comprehensive edit. Where data are determined to be inconsistent, missing, or out of range, NCES contacts the agencies for verification. NCES-prepared state summary forms are returned to the agencies for verification. Each year, states are also given an opportunity to revise their state-level aggregates from the previous survey cycle.

Further information on the nonfiscal CCD may be obtained from

\section{Chen-Su Chen}

Elementary/Secondary and Libraries Studies Division

Elementary/Secondary Cooperative System and Institutional Studies Program

National Center for Education Statistics

1990 K Street NW

Washington, DC 20006

Chen-Su.Chen@ed.gov

http://nces.ed.gov/ccd/ 
Further information on the fiscal CCD data may be obtained from

\section{Stephen Cornman}

Elementary/Secondary and Libraries Studies Division

Elementary/Secondary Cooperative System and Institutional Studies Program

National Center for Education Statistics

1990 K Street NW

Washington, DC 20006

Stephen.Cornman@ed.gov

http://nces.ed.gov/ccd/

\section{Integrated Postsecondary Education Data System}

The Integrated Postsecondary Education Data System (IPEDS) surveys approximately 6,800 postsecondary institutions, including universities and colleges, as well as institutions offering technical and vocational education beyond the high school level. IPEDS, which began in 1986, replaced the Higher Education General Information Survey (HEGIS).

IPEDS consists of nine integrated components that obtain information on who provides postsecondary education (institutions), who participates in it and completes it (students), what programs are offered and what programs are completed, and both the human and financial resources involved in the provision of institutionally based postsecondary education. Until 2000 these components included institutional characteristics, fall enrollment, completions, salaries, finance, and fall staff. Since 2000, data are collected in the fall for institutional characteristics and completions; in the winter for employees by assigned position (EAP), salaries, and fall staff; and in the spring for enrollment, student financial aid, finances, and graduation rates. With the winter 2005-06 survey the employees by assigned position, fall staff, and salaries components were merged into the human resources component. In 2007-08, the enrollment component was broken into two separate components: 12-month enrollment (collected in the fall) and fall enrollment (collected in the spring). The graduation rates 200 percent survey is new to the Spring 2010 collection. Data are collected for the number of students who completed their program within 200 percent of the normal time period. This survey was developed to fulfill requirements in the Higher Education Opportunity Act of 2008.

The degree-granting institutions portion of IPEDS is a census of colleges awarding associate's or higher degrees, that are eligible to participate in Title IV financial aid programs. Prior to 1993, data from technical and vocational institutions were collected through a sample survey. Beginning in 1993, all data were gathered in a census of all postsecondary institutions. The IPEDS tabulations developed for this edition of Projections of Education Statistics are based on lists of all institutions and are not subject to sampling errors.

The definition of institutions generally thought of as offering college and university education has changed in recent years. The old standard for higher education institutions included those institutions that had courses leading to an associate degree or higher, or that had courses accepted for credit toward those degrees. The higher education institutions were accredited by an agency or association that was recognized by the U.S. Department of Education, or were recognized directly by the Secretary of Education. The current category includes institutions that award associate or higher level degrees and that are eligible to participate in Title IV federal financial aid programs. The impact of this change has generally not been large. For example, tables on degrees awarded at the bachelor's level or higher were not heavily affected. Most of the data on public 4-year colleges have been affected only to a minimal extent. The impact on enrollment in public 2-year colleges was noticeable in certain states, but relatively small at the national level. The largest impact has been on private 2-year college enrollment. Overall, total enrollment for all institutions was about one-half of a percent higher for degree-granting institutions than for higher education institutions.

Prior to the establishment of IPEDS in 1986, HEGIS acquired and maintained statistical data on the characteristics and operations of institutions of higher education. Implemented in 1966, HEGIS was an annual universe survey of institutions accredited at the college level by an agency recognized by the Secretary of the U.S. Department of Education. These institutions were listed in the NCES publication Education Directory, Colleges and Universities.

HEGIS surveys collected information concerning institutional characteristics, faculty salaries, finances, enrollment, and degrees. Since these surveys were distributed to all higher education institutions, the data presented are not subject to sampling error. However, they are subject to nonsampling error, the sources of which varied with the survey instrument. Information concerning the nonsampling error of the HEGIS enrollment and degrees surveys can be obtained from the HEGIS Post Survey Validation Study conducted in 1979. 
Further information may be obtained from

\title{
Elise Miller
}

Postsecondary Studies Division

Postsecondary Institutional Studies Program

National Center for Education Statistics

1990 K Street NW

Washington, DC 20006

Elise.Miller@ed.gov

http://nces.ed.gov/ipeds/

Fall (Institutional Characteristics) This survey collects the basic information necessary to classify institutions, including control, level, and types of programs offered, as well as information on tuition, fees, and room and board charges. Beginning in 2000, the survey collected institutional pricing data from institutions with first-time, full-time, degree/certificate-seeking undergraduate students. Unduplicated full-year enrollment headcounts and instructional activity are now collected in a separate component (12-month Enrollment), part of the fall collection. The overall response rate was almost 100 percent for Title IV degree-granting institutions in reporting fall 2009 data.

Further information may be obtained from

\author{
Tara Lawley \\ Postsecondary Studies Division \\ Postsecondary Institutional Studies Program \\ National Center for Education Statistics \\ 1990 K Street NW \\ Washington, DC 20006 \\ Tara.Lawley@ed.gov \\ http://nces.ed.gov/ipeds/
}

Spring (Fall Enrollment) This survey has been part of the HEGIS and IPEDS series since 1966. Response rates for this survey have been relatively high, generally exceeding 85 percent. Beginning in 2000, with web-based data collection, higher response rates were attained. In 2009-10, the overall response rate was over 99.9 percent for degree-granting institutions. The response rate for 4-year private not-for-profit institutions was 99.9 percent, while 4-year public, 4-year private-for-profit, 2-year public, 2-year private not-for-profit, and 2-year private for-profit institutions had response rates of 100.0 percent. Imputation methods and the response bias analysis for the 2008-09 survey are discussed in Knapp (2010).

Public institutions made the majority of changes to enrollment data during the 2004 revision period (Jackson et al. 2005). The majority of changes were made to unduplicated headcount data, with the net differences between the original data and the revised data at about 1 percent. Part-time students in general and enrollment in private not-for-profit institutions were often underestimated. The fewest changes by institutions were to Classification of Instructional Programs (CIP) code data.

Further information about the Spring (Fall Enrollment) survey may be obtained from

\section{Jessica Shedd}

Postsecondary Studies Division

Postsecondary Institutional Studies Program

National Center for Education Statistics

1990 K Street NW

Washington, DC 20006

Jessica.Shedd@ed.gov

http://nces.ed.gov/ipeds/

Fall (Completions) This survey was part of the HEGIS series throughout its existence. Collection of degree data has been maintained through IPEDS. However, the degree classification taxonomy was revised in 1970-71, 1982-83, 1991-92, and 2002-03.

The nonresponse rate does not appear to be a significant source of nonsampling error for this survey. The response rate over the years has been high, with the overall response rate for fall 2009 at 100.0 percent for degree-granting institutions. Because of the high response rate for degree-granting institutions, nonsampling error caused by imputation is also minimal. Imputation methods and the response bias analysis for the fall 2008 survey are discussed in Knapp, Kelly-Reid, and Ginder (2010). 
Most Title IV institutions supplying revised data on completions in 2003-04 were able to supply missing data for the prior year (Jackson et al. 2005). The size of the differences between imputed data for the prior year and the revised actual data supplied by the institution indicated that the imputed values produced by NCES were acceptable.

Further information on the IPEDS Completions surveys may be obtained from

\author{
Andrew Mary \\ Postsecondary Studies Division \\ Postsecondary Institutional Studies Program \\ National Center for Education Statistics \\ 1990 K Street NW \\ Washington, DC 20006 \\ Andrew.Mary@ed.gov \\ http://nces.ed.gov/ipeds/
}

\title{
Private School Universe Survey
}

The purposes of Private School Universe Survey (PSS) data collection activities are to build an accurate and complete list of private schools to serve as a sampling frame for NCES sample surveys of private schools, and to report data on the total number of private schools, teachers, and students in the survey universe. The PSS is conducted every 2 years, with collections in the 1989-90, 1991-92, 1993-94, 1995-96, 1997-98, 1999-2000, 2001-02, 2003-04, 2005-06, 2007-08, and 200910 school years.

The PSS produces data similar to that of the CCD for public schools and can be used for public-private comparisons. The data are useful for a variety of policy and research-relevant issues, such as the growth of religiously affiliated schools, the number of private high school graduates, the length of the school year for various private schools, and the number of private school students and teachers.

The target population for this universe survey is all private schools in the United States that meet the NCES criteria of a school (i.e., a private school is an institution that provides instruction for any of grades $\mathrm{K}$ through 12 , has one or more teachers to give instruction, is not administered by a public agency, and is not operated in a private home). The survey universe is composed of schools identified from a variety of sources. The main source is a list frame, initially developed for the 1989-90 PSS. The list is updated regularly, matching it with lists provided by nationwide private school associations, state departments of education, and other national guides and sources that list private schools. The other source is an area frame search in approximately 124 geographic areas, conducted by the U.S. Census Bureau.

Further information on the PSS may be obtained from

\section{Steve Broughman}

Elementary/Secondary and Libraries Studies Division

Elementary/Secondary Sample Survey Studies Program

National Center for Education Statistics

1990 K Street NW

Washington, DC 20006

Stephen.Broughman@ed.gov

http://nces.ed.gov/surveys/pss/

\section{Census Bureau}

\section{Current Population Survey}

Prior to July 2001, estimates of school enrollment rates, as well as social and economic characteristics of students, were based on data collected in the Census Bureau's monthly household survey of about 50,000 dwelling units. Beginning in July 2001, this sample was expanded to 60,000 dwelling units. The monthly Current Population Survey (CPS) sample consists of 754 areas comprising 2,007 geographic areas, independent cities, and minor civil divisions throughout the 50 states and the District of Columbia. The samples are initially selected based on the decennial census files and are periodically updated to reflect new housing construction.

The monthly CPS deals primarily with labor force data for the civilian noninstitutional population (i.e., excluding military personnel and their families living on post and inmates of institutions). In addition, in October of each year, supplemental questions are asked about highest grade completed, level and grade of current enrollment, attendance status, number and type 
of courses, degree or certificate objective, and type of organization offering instruction for each member of the household. In March of each year, supplemental questions on income are asked. The responses to these questions are combined with answers to two questions on educational attainment: highest grade of school ever attended and whether that grade was completed.

The estimation procedure employed for monthly CPS data involves inflating weighted sample results to independent estimates of characteristics of the civilian noninstitutional population in the United States by age, sex, and race. These independent estimates are based on statistics from decennial censuses; statistics on births, deaths, immigration, and emigration; and statistics on the population in the armed services. Generalized standard error tables are provided in the Current Population Reports or methods for deriving standard errors can be found within the CPS technical documentation at http://www.census.gov/apsd/techdoc/cps/cps-main.html. The CPS data are subject to both nonsampling and sampling errors.

Caution should also be used when comparing data between Census years. With the release of the January 2003 CPS data, population controls that reflect the results of Census 2000 were used in the monthly CPS estimation process. The new controls increased the size of the civilian noninstitutional population by about 3.5 million in May 2002. This adjustment usually occurs 3 to 4 years after the census, and, if the adjustment is substantial, historical data will be revised. Data from January 2000 through December 2002 were revised to reflect these new controls. Over and above these revisions, the U.S. Census Bureau introduced another large upward adjustment to the controls as part of its annual update of population estimates for 2003. The prior change in population controls occurred in March 1993, where data after this date were based on the 1990 census-based population controls and data before this date were based on 1980 or earlier census based population controls. This change in population controls between 1980-based and 1990-based had relatively little impact on summary measures, such as means, medians, and percentage distributions. It does, however, have a significant impact on levels. For example, use of 1990-based population controls resulted in about a 1 percent increase in the civilian noninstitutional population and in the number of families and households. Thus, estimates of levels for data collected in 1994 and later years differed from those for earlier years by more than what could be attributed to actual changes in the population. These differences could be disproportionately greater for certain subpopulation groups than for the total population.

In addition to the changes in population controls, two other relevant changes were introduced into the CPS with the release of the January 2003 data. First, the questions on race and Hispanic origin in the CPS were modified to comply with the new standards for maintaining, collecting, and presenting Federal data on race and ethnicity for Federal statistical agencies. A major change under those standards is that respondents may select more than one race when answering the survey. Respondents continued to be asked a separate question to determine if they are Hispanic or Latino, which is considered an ethnicity rather than a race. The ethnicity question was reworded to ask directly whether the respondent was Hispanic. Persons who report they are Hispanic also are classified separately in the race (or races) they consider themselves to be. Second, improvements were introduced to both the second stage and composite weighting procedures. These changes adapt the weighting procedures to the new race/ethnic classification system and enhance the stability over time of national and state/substate labor force estimates for demographic groups. These two changes, in addition to the change in population controls discussed above, benchmark the CPS data to the results of Census 2000, improve the estimation procedures, and ensure that the data series produced from the survey reflect the evolving composition of the U.S. population.

Further information on CPS may be obtained from

Education and Social Stratification Branch

Population Division

Census Bureau

U.S. Department of Commerce

Washington, DC 20233

http://www.census.gov/cps

School Enrollment Each October, the Current Population Survey (CPS) includes supplemental questions on the enrollment status of the population 3 years old and over, in addition to the monthly basic survey on labor force participation. Prior to 2001, the October supplement consisted of approximately 47,000 interviewed households. Beginning with the October 2001 supplement, the sample was expanded by 9,000 to a total of approximately 56,000 interviewed households. The main sources of non-sampling variability in the responses to the supplement are those inherent in the survey instrument. The question of current enrollment may not be answered accurately for various reasons. Some respondents may not know current grade information for every student in the household, a problem especially prevalent for households with members in college or in nursery school. Confusion over college credits or hours taken by a student may make it difficult to determine the year in which the student is enrolled. Problems may occur with the definition of nursery school (a group or class organized to provide educational experiences for children), where respondents' interpretations of "educational experiences" vary. 
The October 2009 basic CPS household-level response rate was 92.1 percent and the school enrollment supplement personlevel response rate was 93.8 percent. Since these rates are determined at different levels they cannot be combined to derive an overall response rate.

Further information on CPS methodology may be obtained from

http://www.census.gov/cps

Further information on CPS "School Enrollment" may be obtained from

Education and Social Stratification Branch

Census Bureau

U.S. Department of Commerce

Washington, DC 20233

http://www.census.gov/population/www/socdemo/school.html

National Population Projections The 2008 National Population Projections provide projections of resident population and demographic components of change (births, deaths, and net international migration) through 2050. Population projections are available by age, sex, race and Hispanic origin. The following is a general description of the methods used to produce the 2008 National Population Projections.

The projections originated with a base population from Census 2000 and were produced using a cohort-component method. Many of the characteristics of the U.S. resident population, as measured by Census 2000, were preserved as demographic patterns that worked their way through the projection period. Using the cohort-component method, the components of population change (births, deaths, and net international migration) were projected for each birth cohort (persons born in a given year). For each passing year, the population was advanced one year of age. The new age categories were updated using survival rates and levels of net international migration projected for the passing year. A new birth cohort was added to form the population under one year of age by applying projected age-specific fertility rates to the female population aged 15 to 49 , and updating the new cohort for the effects of mortality and net international migration.

The assumptions for the components of change were based on time series analysis. Initially, demographic models were used to summarize historical trends. The forecast parameters obtained from these models were utilized in the models' framework to create fertility, mortality, and migration schedules required for the cohort-component method. Because of limited data about racial characteristics in the fertility and mortality historical series, the assumptions were first developed for three mutually exclusive and exhaustive groups: Hispanic origin (any race), non-Hispanic Black alone, and non-Hispanic all other races. These assumptions were then applied to their respective detailed race/ethnic categories to project the population, allowing presentation of the race categories described above.

Further information on the National Population Projections may be obtained from

Population Division

Census Bureau

U.S. Department of Commerce

Washington, DC 20233

http://www.census.gov

State Population Projections These state population projections were prepared using a cohort-component method by which each component of population change-births, deaths, state-to-state migration flows, international in-migration, and international out-migration - was projected separately for each birth cohort by sex, race, and Hispanic origin. The basic framework was the same as in past Census Bureau projections.

Detailed components necessary to create the projections were obtained from vital statistics, administrative records, census data, and national projections.

The cohort-component method is based on the traditional demographic accounting system:

$$
P_{1}=P_{0}+B-D+D I M-D O M+I I M-I O M
$$


where:

$P_{1}=$ population at the end of the period

$P_{0}=$ population at the beginning of the period

$B=$ births during the period

$D=$ deaths during the period

$D I M=$ domestic in-migration during the period

$D O M=$ domestic out-migration during the period

$I I M=$ international in-migration during the period

$I O M=$ international out-migration during the period

To generate population projections with this model, the Census Bureau created separate datasets for each of these components. In general, the assumptions concerning the future levels of fertility, mortality, and international migration are consistent with the assumptions developed for the national population projections of the Census Bureau.

Once the data for each component were developed the cohort-component method was applied to produce the projections. For each projection year, the base population for each state was disaggregated into eight race and Hispanic categories (non-Hispanic White; non-Hispanic Black; non-Hispanic American Indian, Eskimo, and Aleut; non-Hispanic Asian and Pacific Islander; Hispanic White; Hispanic Black; Hispanic American Indian, Eskimo, and Aleut; and Hispanic Asian and Pacific Islander), by sex, and single year of age (ages 0 to $85+$ ). The next step was to survive each age-sex-race-ethnic group forward 1 year using the pertinent survival rate. The internal redistribution of the population was accomplished by applying the appropriate stateto-state migration rates to the survived population in each state. The projected out-migrants were subtracted from the state of origin and added to the state of destination (as in-migrants). Next, the appropriate number of immigrants from abroad was added to each group. The population under age 1 was created by applying the appropriate age-race-ethnic-specific birth rates to females of childbearing age (ages 15 to 49). The number of births by sex and race/ethnicity were survived forward and exposed to the appropriate migration rate to yield the population under age 1 . The final results of the projection process were proportionally adjusted to be consistent with the national population projections by single years of age, sex, race, and Hispanic origin. The entire process was then repeated for each year of the projection.

More information on Census Bureau projections may be obtained from

Population Division

Census Bureau

U.S. Department of Commerce

Washington, DC 20233

http://www.census.gov

\section{OTHER SOURCES}

\section{IHS Global Insight}

IHS Global Insight provides an information system that includes databases of economic and financial information; simulation and planning models; regular publications and special studies; data retrieval and management systems; and access to experts on economic, financial, industrial, and market activities. One service is the IHS Global Insight Model of the U.S. Economy, which contains annual projections of U.S. economic and financial conditions, including forecasts for the federal government, incomes, population, prices and wages, and state and local governments, over a long-term (10- to 25-year) forecast period.

Additional information is available from

IHS Global Insight

1000 Winter Street

Suite 4300N

Waltham, MA 02451-124

http://www.ihsglobalinsight.com/ 


\section{Appendix D References}

Gamkhar, S., and Oates, W. (1996). Asymmetries in the Response to Increases and Decreases in Intergovernmental Grants: Some Empirical Findings. National Tax Journal, 49(3): pp. 501-512.

Gardner, E.S. (1985). Exponential Smoothing: The State of the Art. Journal of Forecasting, 4(1): pp. 1-28.

Greene, W. (2000). Econometric Analysis. New Jersey: Prentice-Hall.

Hussar, W.J. (1999). Predicting the Need for Newly Hired Teachers in the United States to 2008-09 (NCES 99-026). National Center for Education Statistics, U.S. Department of Education. Washington, DC.

Inman, R.P. (1979). The Fiscal Performance of Local Governments: An Interpretive Review. In P. Mieszkowski and M. Straszheim (Eds.), Current Issues in Urban Economics (pp. 270-321). Baltimore, Maryland: Johns Hopkins Press.

Intriligator, M.D. (1978). Econometric Models, Techniques, \& Applications. New Jersey: Prentice-Hall, Inc.

IHS Global Insight, U.S. Monthly Model November 2009: Short-Term Projections.

Jackson, K.W., Jang, D., Sukasih, A., and Peeckson, S. (2005). Integrated Postsecondary Education Data System Data Quality Study (NCES 2005-175). National Center for Education Statistics, Institute of Education Sciences, U.S. Department of Education. Washington, DC.

Johnston, J., and Dinardo, J. (1996). Econometric Methods. New York: McGraw-Hill.

Judge, G., Hill, W., Griffiths, R., Lutkepohl, H., and Lee, T. (1985). The Theory and Practice of Econometrics. New York: John Wiley and Sons.

Knapp, L.G. (2010). Postsecondary Institutions and Price of Attendance in the United States: Fall 2009 and Degrees and Other Awards Conferred: 2008-09, and 12-Month Enrollment 2008-09 (NCES 2009-165). National Center for Education Statistics, Institute of Education Sciences, U.S. Department of Education. Washington, DC.

Knapp, L.G., Kelly-Reid, J.E., and Ginder, S.A. (2010). Enrollment in Postsecondary Institutions, Fall 2008; Graduation Rates, 2002 \& 2005 Cohorts; and Financial Statistics, Fiscal Year 2008 (NCES 2010-152REV). National Center for Education Statistics, Institute of Education Sciences, U.S. Department of Education. Washington, DC.

Makridakis, S., Wheelwright, S.C., and Hyndman, R.J. (1998). Forecasting: Methods and Applications (3rd ed.). New York: John Wiley \& Sons, Inc.

Mitias, P., and Turnbull, G. (2001). Grant Illusion, Tax Illusion, and Local Government Spending. Public Finance Review, 29(5): pp. 347-368.

U.S. Department of Commerce, Census Bureau, 2008 National Population Projections, retrieved November 2, 2009, from http://www.census.gov/population/www/projections/2008projections.html.

U.S. Department of Commerce, Census Bureau, State Interim Population Projections, retrieved November 2, 2009, from http://www.census.gov/population/www/projections/projectionsagesex.html. 
This page intentionally left blank. 


\title{
Appendix E List of Abbreviations
}

\author{
ADA Average daily attendance \\ BLS Bureau of Labor Statistics \\ CCD Common Core of Data \\ CIP Classification of Instructional Programs \\ CPI Consumer Price Index \\ CPS Current Population Survey \\ EAP Employees by assigned position
}

EDMOD Education Forecasting Model

FTE Full-time-equivalent

HEGIS Higher Education General Information Survey

IPEDS Integrated Postsecondary Education Data System

LEA Local education agency

MAPE Mean absolute percentage error

NCES National Center for Education Statistics

NCHS National Center for Health Statistics 
This page intentionally left blank. 


\section{Appendix F Glossary}

American Indian/Alaska Native: A person having origins in any of the original peoples of North America and who maintains cultural identification through tribal affiliation or community recognition.

Asian/Pacific Islander: A person having origins in any of the original peoples of the Far East, Southeast Asia, the Indian Subcontinent, and Pacific Islands. This includes people from China, Japan, Korea, the Philippine Islands, American Samoa, India, and Vietnam.

Associate's degree: An award that normally requires at least 2 but less than 4 years of full-time equivalent college work.

Autocorrelation: Correlation of the error terms from different observations of the same variable. Also called serial correlation.

Average daily attendance (ADA): The aggregate attendance of a school during a reporting period (normally a school year) divided by the number of days school is in session during this period. Only days on which the pupils are under the guidance and direction of teachers should be considered days in session.

Average daily membership (ADM): The aggregate membership of a school during a reporting period (normally a school year) divided by the number of days school is in session during this period. Only days on which the pupils are under the guidance and direction of teachers should be considered as days in session. The ADM for groups of schools having varying lengths of terms is the average of the ADMs obtained for the individual schools.

Bachelor's degree: An award (baccalaureate or equivalent degree, as determined by the Secretary, U.S. Department of Education) that normally requires at least 4 but not more than 5 years of full-time equivalent college-level work. This includes all bachelor's degrees conferred in a 5-year cooperative (work-study) program. A cooperative plan provides for alternate class attendance and employment in business, industry, or government; thus, it allows students to combine actual work experience with their college studies. Also includes bachelor's degrees in which the normal 4 years of work are completed in 3 years.

Black: A person having origins in any of the black racial groups of Africa (except those of Hispanic origin).

Breusch-Godfrey serial correlation LM test: A statistic testing the independence of errors in least-squares regression against alternatives of first-order and higher degrees of serial correlation. The test belongs to a class of asymptotic tests known as the Lagrange multiplier (LM) tests.

Classroom teacher: A staff member assigned the professional activities of instructing pupils in self-contained classes or courses, or in classroom situations. Usually expressed in fulltime-equivalents.

Cohort: A group of individuals that have a statistical factor in common (e.g., year of birth).

Cohort-component method: A method for estimating and projecting a population is distinguished by its ability to preserve knowledge of an age distribution of a population (which may be of a single sex, race, and Hispanic origin) over time.

College: A postsecondary school that offers a general or liberal arts education, usually leading to an associate's, bachelor's, master's, doctor's, or first-professional degree. Junior colleges and community colleges are included in this term.

Constant dollars: Dollar amounts that have been adjusted by means of price and cost indexes to eliminate inflationary factors and allow direct comparison across years.

Consumer Price Index (CPI): This price index measures the average change in the cost of a fixed-market basket of goods and services purchased by consumers.

Current dollars: Dollar amounts that have not been adjusted to compensate for inflation.

Current expenditures (elementary/secondary): The expenditures for operating local public schools and school districts, excluding capital outlay, interest on school debt, and programs outside of public elementary and secondary education. These expenditures include such items as salaries for school personnel, fixed charges, student transportation, school books and materials, and energy costs.

Current expenditures per pupil in average daily attendance (ADA): Current expenditures for the regular school term divided by the ADA of full-time pupils (or full-timeequivalency of pupils) during the term. See also Current expenditures and Average daily attendance.

Current Population Survey: See appendix C, Data Sources.

Degree-granting institutions: Postsecondary institutions that are eligible for Title IV federal financial aid programs and that 
grant an associate's or higher degree. For an institution to be eligible to participate in Title IV financial aid programs it must offer a program of at least 300 clock hours in length, have accreditation recognized by the U.S. Department of Education, have been in business for at least 2 years, and have signed a participation agreement with the Department.

Degrees of freedom: The number of free or linearly independent sample observations used in the calculation of a statistic. In a time series regression with $t$ time periods and $k$ independent variables including a constant term, there would be $t$ minus $k$ degrees of freedom.

Dependent variable: A mathematical variable whose value is determined by that of one or more other variables in a function. In regression analysis, when a random variable, $y$, is expressed as a function of variables $x_{1}, x_{2}, \ldots x_{k}$, plus a stochastic term, then $y$ is known as the "dependent variable."

Disposable income: Current income received by persons less their contributions for social insurance, personal tax, and nontax payments. It is the income available to persons for spending and saving. Nontax payments include passport fees, fines and penalties, donations, and tuitions and fees paid to schools and hospitals operated mainly by the government. See also Personal income.

Doctor's degree: The highest award a student can earn for graduate study. The doctor's degree classification includes such degrees as Doctor of Education, Doctor of Juridical Science, Doctor of Public Health, and the Doctor of Philosophy degree in any field such as agronomy, food technology, education, engineering, public administration, ophthalmology, or radiology.

Double exponential smoothing: A method that takes a single smoothed average component of demand and smoothes it a second time to allow for estimation of a trend effect.

Durbin-Watson statistic: A statistic testing the independence of errors in least squares regression against the alternative of first-order serial correlation. The statistic is a simple linear transformation of the first-order serial correlation of residuals and, although its distribution is unknown, it is tested by bounding statistics that follow R. L. Anderson's distribution.

Econometrics: The quantitative examination of economic trends and relationships using statistical techniques, and the development, examination, and refinement of those techniques.

Elementary and secondary schools: As used in this publication, includes only regular schools, that is, schools that are part of state and local school systems and also most private elementary and secondary schools, both religiously affiliated and nonsectarian. Schools not included in this term are subcollegiate departments of institutions of higher education, federal schools for Indians, and federal schools on military posts and other federal installations.
Elementary school: A school classified as elementary by state and local practice and composed of any span of grades not above grade 8 . A preschool or kindergarten school is included under this heading only if it is an integral part of an elementary school or a regularly established school system.

Enrollment: The number of students registered in a given school unit at a given time, generally in the fall of a year.

Estimate: A numerical value obtained from a statistical sample and assigned to a population parameter. The particular value yielded by an estimator in a given set of circumstances or the rule by which such particular values are calculated.

Estimating equation: An equation involving observed quantities and an unknown that serves to estimate the latter.

Estimation: Estimation is concerned with inference about the numerical value of unknown population values from incomplete data, such as a sample. If a single figure is calculated for each unknown parameter, the process is called point estimation. If an interval is calculated within which the parameter is likely, in some sense, to lie, the process is called interval estimation.

Expenditures: Charges incurred, whether paid or unpaid, that are presumed to benefit the current fiscal year. For elementary and secondary schools, these include all charges for current outlays plus capital outlays and interest on school debt. For degree-granting institutions, these include current outlays plus capital outlays. For government, these include charges net of recoveries and other correcting transactions other than for retirement of debt, investment in securities, or extension of credit. Government expenditures include only external transactions, such as the provision of perquisites or other payments in kind. Aggregates for groups of governments exclude intergovernmental transactions.

Expenditures per pupil: Charges incurred for a particular period of time divided by a student unit of measure, such as average daily attendance or average daily membership.

Exponential smoothing: A method used in time series analysis to smooth or to predict a series. There are various forms, but all are based on the supposition that more remote history has less importance than more recent history.

First-order serial correlation: When errors in one time period are correlated directly with errors in the ensuing time period. Also called autocorrelation.

First-professional degree: An award that requires completion of a program that meets all of the following criteria: (1) completion of the academic requirements to begin practice in the profession; (2) at least 2 years of college work prior to entering the program; and (3) a total of at least 6 academic years of college work to complete the degree program, including prior required college work plus the length of the professional program itself. Firstprofessional degrees may be awarded in the following 10 fields: Chiropractic (D.C. or D.C.M.), Dentistry (D.D.S. or D.M.D.), 
Law (L.L.B., J.D.), Medicine (M.D.), Optometry (O.D.), Osteopathic Medicine (D.O.), Pharmacy (Pharm.D.), Podiatry (D.P.M., D.P., or Pod.D.), Theology (M.Div., M.H.L., B.D., or Ordination), Veterinary Medicine (D.V.M.).

First-professional enrollment: The number of students enrolled in following degree programs: Chiropractic (D.C. or D.C.M.), Dentistry (D.D.S. or D.M.D.), Law (L.L.B., J.D.), Medicine (M.D.), Optometry (O.D.), Osteopathic Medicine (D.O.), Pharmacy (Pharm.D.), Podiatry (D.P.M., D.P., or Pod.D.), Theology (M.Div., M.H.L., B.D., or Ordination), Veterinary Medicine (D.V.M.).

First-time freshman: A student attending any institution for the first time at the undergraduate level. Includes students enrolled in academic or occupational programs. Also includes students enrolled in the fall term who attended college for the first time in the prior summer term, as well as students who entered with advanced standing (college credits earned before graduation from high school).

Forecast: An estimate of the future based on rational study and analysis of available pertinent data, as opposed to subjective prediction.

Forecasting: Assessing the magnitude that a quantity will assume at some future point in time, as distinct from "estimation," which attempts to assess the magnitude of an already existent quantity.

Four-year institution: A postsecondary institution that offers programs of at least 4 years duration or one that offers programs at or above the baccalaureate level. Includes schools that offer postbaccalaureate certificates only or those that offer graduate programs only. Also includes free-standing medical, law or other first-professional schools.

Full-time-equivalent (FTE) enrollment: A measurement equal to one student enrolled full time for one academic year. Total FTE enrollment includes full time plus the calculated equivalent of the part-time enrollment. The full-time equivalent of the part-time students can be estimated using different factors depending on the type and control of institution and level of student.

Full-time worker: In educational institutions, an employee whose position requires being on the job on school days throughout the school year at least the number of hours the schools are in session; for higher education, a member of an educational institution's staff who is employed full time.

Function: A mathematical correspondence that assigns exactly one element of one set to each element of the same or another set. A variable that depends on and varies with another.

Functional form: A mathematical statement of the relationship among the variables in a model.
Graduate: An individual who has received formal recognition for the successful completion of a prescribed program of studies.

Graduate enrollment: The number of students who hold the bachelor's or first-professional degree, or the equivalent, and who are working towards a master's or doctor's degree. First-professional students are counted separately. These enrollment data measure those students who are registered at a particular time during the fall.

Higher education: Study beyond secondary school at an institution that offers programs terminating in an associate's, baccalaureate, or higher degree.

\section{Higher education institutions (traditional classifications):}

4-year institution: An institution legally authorized to offer and offering at least a 4-year program of collegelevel studies wholly or principally creditable toward a bachelor's degree. A university is a postsecondary institution that typically includes one or more graduate professional schools.

2-year institution: An institution legally authorized to offer and offering at least a 2-year program of collegelevel studies that terminates in an associate's degree or is principally creditable toward a baccalaureate.

See also Degree-granting institutions and Postsecondary education.

High school: A secondary school offering the final years of high school work necessary for graduation, usually including grades 10,11 , and 12 (in a 6-3-3 plan) or grades 9, 10, 11, and 12 (in a 6-2-4 plan).

Hispanic: A person of Mexican, Puerto Rican, Cuban, Central or South American or other Spanish culture or origin, regardless of race.

Independent variable: In regression analysis, a random variable, $y$, is expressed as a function of variables $x_{l}, x_{2}, \ldots x_{k}$, plus a stochastic term; the $x$ 's are known as "independent variables."

\section{Interpolation: See Linear interpolation.}

Lag: An event occurring at time $t+k(k>0)$ is said to lag behind an event occurring at time $t$, the extent of the lag being $k$. An event occurring $k$ time periods before another may be regarded as having a negative lag.

Linear interpolation: A method that allows the prediction of an unknown value if any two particular values on the same scale are known and the rate of change is assumed constant.

Master's degree: An award that requires the successful completion of a program of study of at least the full-time equivalent of 1 but not more than 2 academic years of work beyond the bachelor's degree. 
Mean absolute percentage error (MAPE): The average value of the absolute value of errors expressed in percentage terms.

Model: A system of postulates, data, and inferences presented as a mathematical description of a phenomenon, such as an actual system or process. The actual phenomenon is represented by the model in order to explain, predict, and control it.

Nonresident alien: A person who is not a citizen or national of the United States and who is in this country on a visa or temporary basis and does not have the right to remain indefinitely.

Ordinary least squares (OLS): The estimator that minimizes the sum of squared residuals.

Parameter: A quantity that describes a statistical population.

Part-time enrollment: Undergraduate-A student enrolled for either 11 semester credits or less, or 11 quarter credits or less, or less than 24 contact hours a week each term. Graduate-A student enrolled for either 8 semester credits or less, or 8 quarter credits or less.

Personal income: Current income received by persons from all sources minus their personal contributions for social insurance. Classified as "persons" are individuals (including owners of unincorporated firms), nonprofit institutions serving individuals, private trust funds, and private noninsured welfare funds. Personal income includes transfers (payments not resulting from current production) from government and business such as social security benefits, military pensions, and so forth, but excludes transfers among persons.

Postbaccalaureate enrollment: The number of students with a bachelor's degree who are enrolled in graduate-level or firstprofessional courses.

Postsecondary education: The provision of a formal instructional program whose curriculum is designed primarily for students who are beyond the compulsory age for high school. This includes programs whose purpose is academic, vocational, and continuing professional education, and excludes avocational and adult basic education programs.

Postsecondary education institution: An institution which has as its sole purpose or one of its primary missions, the provision of postsecondary education.

Private institution: A school or institution that is controlled by an individual or agency other than a state, a subdivision of a state, or the federal government (i.e., usually supported primarily by other than public funds) and the operation of whose program rests with other than publicly elected or appointed officials.

Projection: In relation to a time series, an estimate of future values based on a current trend.
Property tax: The sum of money collected from a tax levied against the value of property.

Public school or institution: A school or institution controlled and operated by publicly elected or appointed officials, and generally deriving its primary support from public funds.

Pupil/teacher ratio: The enrollment of pupils at a given period of time, divided by the full-time-equivalent number of classroom teachers serving these pupils during the same period.

$\boldsymbol{R}^{2}$ : The coefficient of determination; the square of the correlation coefficient between the dependent variable and its OLS estimate.

Race/ethnicity: Categories used to describe groups to which individuals belong, identify with, or belong in the eyes of the community. The categories do not denote scientific definitions of anthropological origins. A person may be counted in only one group. The groups used to categorize U.S. citizens, resident aliens, and other eligible non-citizens in this report are as follows: Black, American Indian/Alaska Native, Asian/Pacific Islander, Hispanic, White.

Region: The four geographical regions of the United States as defined by the Census Bureau of the U.S. Department of Commerce presented below:

Northeast: Connecticut, Maine, Massachusetts, New Hampshire, New Jersey, New York, Pennsylvania, Rhode Island, and Vermont.

Midwest: Illinois, Indiana, Iowa, Kansas, Michigan, Minnesota, Missouri, Nebraska, North Dakota, Ohio, South Dakota, and Wisconsin.

South: Alabama, Arkansas, Delaware, District of Columbia, Florida, Georgia, Kentucky, Louisiana, Maryland, Mississippi, North Carolina, Oklahoma, South Carolina, Tennessee, Texas, Virginia, and West Virginia.

West: Alaska, Arizona, California, Colorado, Hawaii, Idaho, Montana, Nevada, New Mexico, Oregon, Utah, Washington, and Wyoming.

Regression analysis: A statistical technique for investigating and modeling the relationship between variables.

Revenue receipts: Additions to assets that do not incur an obligation that must be met at some future date and do not represent exchanges of property for money. Assets must be available for expenditures.

Revenues: All funds received from external sources, net of refunds and correcting transactions. Noncash transactions such as receipt of services, commodities, or other receipts "in kind" are excluded, as are funds received from the issuance of debt, liquidation of investments, or nonroutine sale of property. 
Rho: A measure of the correlation coefficient between errors in time period $t$ and time period $t$ minus 1 .

Salary: The total amount regularly paid or stipulated to be paid to an individual, before deductions, for personal services rendered while on the payroll of a business or organization.

School: A division of the school system consisting of students in one or more grades or other identifiable groups and organized to give instruction of a defined type. One school may share a building with another school or one school may be housed in several buildings.

Secondary instructional level: The general level of instruction provided for pupils in secondary schools (generally covering grades 7 through 12 or 9 through 12), and any instruction of a comparable nature and difficulty provided for adults and youth beyond the age of compulsory school attendance.

Secondary school: A school including any span of grades beginning with the next grade following elementary or middle school (usually 7, 8, or 9) and ending with or below grade 12 . Both junior high schools and senior high schools are included.

Senior high school: A secondary school offering the final years of high school work necessary for graduation.

Serial correlation: Correlation of the error terms from different observations of the same variable. Also called autocorrelation.

Standard error of estimate: An expression for the standard deviation of the observed values about a regression line. An estimate of the variation likely to be encountered in making predictions from the regression equation.

Student: An individual for whom instruction is provided in an educational program under the jurisdiction of a school, school system, or other educational institution. No distinction is made between the terms "student" and "pupil," although "student" may refer to one receiving instruction at any level while "pupil" refers only to one attending school at the elementary or secondary level. The term "student" is used to include individuals at all instructional levels. A student may receive instruction in a school facility or in another location, such as at home or in a hospital. Instruction may be provided by direct student-teacher interaction or by some other approved medium, such as the Internet, television, radio, telephone, or correspondence.

Tax base: The collective value of sales, assets, and income components against which a tax is levied.

Time series: A set of ordered observations on a quantitative characteristic of an individual or collective phenomenon taken at different points in time. Usually the observations are successive and equally spaced in time.

Time series analysis: The branch of quantitative forecasting in which data for one variable are examined for patterns of trend, seasonality, and cycle.

Two-year institution: A postsecondary institution that offers programs of at least 2 but less than 4 years duration. Includes occupational and vocational schools with programs of at least 1800 hours and academic institutions with programs of less than 4 years. Does not include bachelor's degreegranting institutions where the baccalaureate program can be completed in 3 years.

Undergraduate enrollment: The number of students enrolled in a 4- or 5-year bachelor's degree program, an associate's degree program, or a vocational or technical program below the baccalaureate.

Undergraduate students: Students registered at an institution of higher education who are working in a program leading to a baccalaureate or other formal award below the baccalaureate, such as an associate's degree.

Ungraded student (elementary/secondary): A student who has been assigned to a school or program that does not have standard grade designations.

Variable: A quantity that may assume any one of a set of values.

White: A person having origins in any of the original peoples of Europe, North Africa, or the Middle East (except those of Hispanic origin). 